\title{
Severity of illness and costs of medical care in patients with acute myocardial infarction
}

Citation for published version (APA):

Voss, G. B. W. E. (1993). Severity of illness and costs of medical care in patients with acute myocardial infarction. [Doctoral Thesis, Maastricht University]. Datawyse / Universitaire Pers Maastricht. https://doi.org/10.26481/dis.19930205gv

Document status and date:

Published: 01/01/1993

DOI:

10.26481/dis.19930205gv

Document Version:

Publisher's PDF, also known as Version of record

\section{Please check the document version of this publication:}

- A submitted manuscript is the version of the article upon submission and before peer-review. There can be important differences between the submitted version and the official published version of record.

People interested in the research are advised to contact the author for the final version of the publication, or visit the DOI to the publisher's website.

- The final author version and the galley proof are versions of the publication after peer review.

- The final published version features the final layout of the paper including the volume, issue and page numbers.

Link to publication

\footnotetext{
General rights rights.

- You may freely distribute the URL identifying the publication in the public portal. please follow below link for the End User Agreement:

www.umlib.nl/taverne-license

Take down policy

If you believe that this document breaches copyright please contact us at:

repository@maastrichtuniversity.nl

providing details and we will investigate your claim.
}

Copyright and moral rights for the publications made accessible in the public portal are retained by the authors and/or other copyright owners and it is a condition of accessing publications that users recognise and abide by the legal requirements associated with these

- Users may download and print one copy of any publication from the public portal for the purpose of private study or research.

- You may not further distribute the material or use it for any profit-making activity or commercial gain

If the publication is distributed under the terms of Article $25 \mathrm{fa}$ of the Dutch Copyright Act, indicated by the "Taverne" license above, 
Severity of Illness and Costs of Medical Care in patients with Acute Myocardial Infarction. 


\title{
severity of illness and costs of medical care in patients with acute myocardial infarction
}

\section{proefschrift}

\author{
ter verkrijging van de graad van doctor \\ aan de Rijksuniversiteit Limburg te Maastricht, \\ op gezag van de Rector Magnificus, Prof. Mr. M.J. Cohen, \\ volgens het besluit van het College van Dekanen, \\ in het openbaar te verdedigen \\ op vrijdag 5 februari 1993 om 14.00 uur
}

door

Gemma Benedicta Wilhelmina Elisabeth Voss

geboren te Susteren in 1961

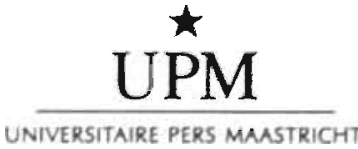




\section{Promotores:}

Prof. Dr. F.F.H. Rutten.

Prof. Dr. Ir. A. Hasman.

Co-promotores:

Dr. F.H.M. Nieman.

Dr. C. de Zwaan.

Beoordelingscommissie:

Prof. Dr. R.M. Leidl (voorzitter).

Prof. Dr. J.D.F. Habbema.

Prof. Dr. J.M.J.P. van der Linden.

Prof. Dr. A.P.W.P. van Montfort.

Prof. Dr. H.J.J. Wellens.

CIP-DATA KONINKLIJKE BIBLIOTHEEK, DEN HAAG

Voss, Gemma Benedicta Wilhelmina Elisabeth

Severity of illness and costs of medical care in patients

with acule myocardial infarction/

Gemma Benedicta Wilhelmina Elisabeth Voss - Maastricht: Universitaire

Pers Maastricht. - IIl.

Thesis Maastrichl- With ref.

ISBN 90-5278-054-4

NUGI $742 / 689$

Subject Headings: acute myocardial infarction patients/ severity of illness/medical care; costs,

\section{Produktie:}

Datawyse, Maastricht

Lay-out:

Hetly Creemers, Maastricht

Omslagontwerp:

W. Verheijden - Pustjens

Druk:

Krips Repro B.V.. Meppel

Financial support for the publication of this thesis is gratefully acknowledged by: the Netherlands Heart Foundation. SIG-Health Care Information, Utrecht and $3 \mathrm{M}$ Nederland B.V.

Het verschijnen van dit proefschrift werd mede mogelijk gemaakt door de steun van: de Nederlandse Hartstichting, SIG -Zorginformatie, Utrecht en 3M Nederland B.V. 
voor mijn ouders 



\section{Contents.}

Preface 11

1. Introduction 13

2. Patient classification systems: a review 17

2.1. Theoretical background of patient classification systems 17

2.2. Review of patient classification systems 19

2.2.1. Diagnosis Related Groups 20

2.2.2. Patient Management Categories $\quad 22$

2.2.3. Disease Staging 23

2.2.4. Computerized Severity of Illness 25

2.2.5. Acute Physiology And Chronic Health Evaluation 25

2.2.6. The Medical Illness Severity Grouping System 27

2.3. Evaluative comparison of patient classification systems 29

3. Explanation of medical care costs $\quad 35$

3.1. Study objective and definition of problem 35

3.2. Factors which explain differences in medical care costs 37

3.3. Models for explaining differences in medical care costs 39

3.4. Adaptation of the models to patients with Acute Myocardial
Infarction (AMI)

4. Patient population and data collection 43

4.1. Patient population 43

4.2 Hospitalization characteristics of the patient population 43

4.3. Data collection 45

4.4. Quality of data 46

5. Medical care costs and severity of illness: definitions and opcrationalization

5.1. Costs of medical care in hospitals 49

5.1.1. Cost allocation of departmental costs to medical care
services

5.2. Severity of Illness 55

5.2.1. Assumptions and terminology regarding severity of
illiness in AMI patients

5.2.2. Severity of Acute Myocardial Infarction: a causal model 57

5.2.3. Defining Severity of Illness: an operationalization 58

5.2.4. Severity of AMI patients during hospital stay; changes in severity and rules to obtain dynamic Severity of Illness Categories $\quad 60$

5.2.5. Summary 62

6. Measurement of Research Concepts 63

6.1. Costs of medical care. 63

6.1.1. Costs of diagnostic services 64

6.1.2. Costs of medical interventions 65

6.1.3. Costs of nursing care 66

$\begin{array}{ll}\text { 6.1.4. Other costs } & 67 \text {. }\end{array}$ 
6.2. Severity of Illness of patients having AMI 68

6.2.1. Severity of coronary stenosis 69

6.2.2. Severity of myocardial ischemia 73

6.2.2.1. Severity based on electrocardiographic ST-changes al rest

6.2.2.2. Severity based on electrocardiographic ST-changes during exercise

6.2.2.3. Severity based on reversible thallium changes 78

6.2.3. Severity of myocardial necrosis

6.2.3.1. Severity based on electrocardiographic

Q-waves in the myocardial wall segments

6.2.3.2. Severity of myocardial necrosis measured by enzyme changes in the blood

6.2.3.3. Severity of necrosis measured by irreversible thallium changes

6.2.4. Severity of ischemic pain

6.2.5. Severity of cardiac wall motion abnormalities 89

6.2.6. Severity of cardiac rhythm disorders 91

6.2.7. Severity of cardiac conduction disorders 94

6.2.8. Severity of heart failure 95

6.2.8.1. Severity of heart failure in the left ventricle 96

6.2.8.2. Severity of heart failure in the right ventricle 97

6.2.9. Severity of physical deficiencies and specific cardiac complications

$\begin{array}{ll}\text { 6.3. Background characteristics of patients } & 100 \\ \text { 6.3.1. Individual back ground characteristics } & 100\end{array}$

6.3.2. Contra-indications 102

6.3.3. Enrollment of patients in a hospital research program 102

7. Investigating hypothesized relations between severity of illness and costs of medical care

7.1. Methods used in data analysis 103

7.2. Period specific cost analysis 110

7.2.1. Explanation of costs of thrombolytic therapy 110

7.2.2. Explanation of costs due to (acute) heart catheterization 11.2

7.2.3. Explanation of costs due to (acute) PTCA 115

7.3. Analysis of length of stay and costs in medical care during total
hospital stay

7.3.1. Explanation of length of stay $\quad 119$

7.3.2. Explanation of costs of the coronary care unit 130

7.3.3. Explanation of other costs of medical care $\quad 140$

7.4. Conclusions 143

8. Classifying Acute Myocardial Infarction patients according to their

Severity of lliness

8.1. Comparison of severity indicators empirically found in this study and assumptions of other studies

8.2. Severity of Illness Classification (SIC) for AMI patients 150

8.3. Comparison of the predictive validity of the Severity of Illnessclassification and that of Diagnosis Related Groups-classification $\quad 158$

8.4. Applications of SIC-LOS 162

8.5. Conclusions 164 
9. Conclusions and discussion

9.1. Conclusions

9.2. Discussion

Summary

Samenvatting

Glossary

Bibliography 183

Appendices 192

Curriculum Vitae 



\section{Preface.}

A question like :" What is the effect of a patient's severity of illness on the costs of medical care?" can not be answered without the help of numerous people. During the past five years I had the privilege of working with many different professionals who each have contributed to this study in their own way.

All this would not have been possible without the support of the Board of Directors of the University Hospital Maastricht. Thanks to the progressive attitude towards patient oriented management information of Lou Brans-Brabant and Jan Carpay, 1 had the opportunity to develop and implement the DRG-system in this hospital and at the same time do this study.

Over the years, my supervisors Frans Rutten and Arie Hasman always showed a great interest in the study. Furihermore, they gave me valuable ideas and suggestions that improved earlier versions of this book.

I am especially grateful to Fred Nieman who guided and helped me during ali stages of the research with his unremitting commitment, support and critical comments. He acquainied me in particular with the pitfalls in the fielus of methodology and statistics. Besides his support, I always appreciated his Belgium chocolates.

My special thanks must also go to Chris de Zwaan who, despite his demanding job, could always find the time to clarify my often numerous clinical questions. I have enjoyed the pleasant way in which he provided me with critical comments and insightful guidance within the difficult fields of cardiology.

I am also very grateful for the contributions from many other cardiologists like Frans Pieters, Simon Braat, and Frank Vermeer from the Department of Cardiology at the University Hospital Maastricht and Hans Bennier, from the Department of Cardiology at the Catharina Hospital Eindhoven who have heiped $\mathrm{me}$ in the conceptualization, data collection, determination of severity weights and interpretation of results. I would furthermore like to thañk Wim Hermens who helned a great deal by guiding us through the complexities of the biochemical part of this study.

I have benefitted gratefully from the work of the economists Pascal Limpens, Serve Roberts, Victor Kamm and numerous students who have carricd out cost allocation studies at the ancillary departments of the University Hospital Maastricht. I did not meet all students personally, but I am very appreciative of all the work they have been doing.

The medical data collection was a time-consuming job which was conscientiously carried out by Kay Liedekerken. Marlie Burger and Gys Hendriks under the expert supervision of Frans Pieters. The database-program was developed by Reinhilde Silkens and Vic: Dreessen working at the Department of Medical Informatics and Statistics of the University of Limburg. Also, I have received a lot of support from the computer department from the University Hospital Maastricht, especially from Richard Schurgers who helped me in extracting the discharge and billing data from the hospital information system.

The final version of this book was a joint effort by Jenny Aussems-Maas who has word processed and reprocessed the many versions of the manuscript with a remarkable patience, Brenda Swaak who has edited the text into proper English, Wilma Verheyden-Pustjens who has designed the book cover and Hetty Creemers who took care of the lay-out. 
A lot of other people not directly involved in this study have created a stimulating environment in which I could work on this thesis.

I could always rely on the support and interest of my colleagues who have shared in the ups and downs of the project.

I thank my friends for having a lot of patience and sympathy even during the moments that I could not give them the attention I would have liked.

Although my parents and family remained in the background, their warmth and care was always very close to me.

A very special place in all of this is for Sjeng. His positive attitude, his understanding and his music have meant a lot to me. 
A hospital can be compared with a multi-product organization, in which a variety of outputs -in the sense of 'medical care products' provided to patients- is produced. Health care providers and hospital managers are responsible for the effectiveness and efficiency of the medical care provided. Today, cost control is an important issue in health care policy. Information on the quality of hospital processes and the outcomes of these processes in relation to the money spent has become a necessity for modern hospital managers. Furthermore, information on factors influencing hospital costs is essential for controlling hospital costs. Hornbrook (1982) has lucidly described the relationship between hospital costs, health care policy, hospital management and patient care: 'The definition and measurement of the output of the hospital is a complex, persistent and pervasive problem. It is a central concern to health policy makers, who must determine ways of improving the reimbursement mechanisms to provide greater incentives for cost-effective operations of hospitals; to hospital administrators, who must develop ever more accurate forecasts of staffing and budget needs in an era of increased cost-consciousness; and to health services researchers, who must develop better measures of hospital output to advance our understanding of the determinants of hospital productivity and costliness. To achieve these goals of predicting, explaining and controlling hospital performance, it is necessary to define what the hospital produces and to specify appropriate measures. However, quick resolution of the measurement problem is precluded by the complex, multidimensional nature of hospital activities, and the multiple objectives of the physicians and patients who use the hospital'.

Variables to operationalize the hospital output have been proposed in a variety of research seltings. Variables representing hospital facilities and services like number of beds, high care facilities, ambulatory services, mix of medical specialties etc. have frequently been used in studies to explore cost differences among hospitals (Berry 1970, Berry 1973, Ruchlin 1974, Van Aert 1977, Van Montfort 1980). However, using these variables places more emphasis on the characteristics of a hospital organization than on the complexity of the hospital patient population. Therefore, this approach can be regarded as an easily available surrogate for measuring hospital output. Other studies have shown that variables reflecting the hospital case-mix, like number of patients in specific diagnostic groups, surgical groups and age/sex categories, describe hospital output in a different way. Consequently, case-mix variables will offer a more precise explanation for differences in costs of medical care (Evans 1971, Lave 1971, Feldstcin 1977, Watts 1980, Klastorin 1980).

Many researchers have dealt with the problem of finding factors that explain cost differences between and within hospitals. Although they have come to the conclusion that case-mix variables, like diagnoses and surgical procedures, are more successful in explaining cost differences than indirect variables describing hospital characteristics such as patient days, number of beds, hospital facilities etc., they have at the same time reported important difficulties in measuring the hospital case-mix in an adequate way. First of all, a hospital population must be defined in terms of patient categories which on the one hand makes sense to physicians and on the other contains economic relevancy for hospital managers. Medically relevant patient categories must be homogeneous with respect to costs: patients who have similar costs patterns must be defined within the same category. Only then, can the hospital output be defined and measured in an adequate way.

In the past decade several studies have concentrated on the development of patient classification systems that aim to explain differences in costs between and within hospitals 
(see chapter 2). Such patient classification systems may be applied to several areas within health care (Groot 1978, Fetter 1980, Nederstigt 1981, Wood 1981, Hornbrook 1982, Grimaldi 1983 . Fetter 1986, Lichting 1986).

\section{Reimbursement of hospital services.}

A very well-known patient classification is the Diagnosis Related Group (DRGi)-system which is used as a prospective reimbursement system for hospitals in the US health care system. To finance patient care within hospitals, specific reimbursement rates have been established for each DRG separately. Desharnais (1987), and Rosko (1987) have come to the conclusion that this type of prospective payment system reduces hospital utilization without resulting in a deterioration in the quality of care. Prince (1987) and Wyszewianski e.a. (1987) conclude that an advantage of this form of payment is that it provides an incentive for better hospital management ('Managed Care'). On the other hand, adverse consequences for the US health care system may be that this type of system provides incentives to choose surgical over medical treatment, or that it leads to selective admission practices or to an increase in re-admissions (Omenn e.a. 1984, Hunt 1988, Stein 1985, Torf 1989). Roger e.a. (190()) found that mortality following hospitalization has been unaffected, and improvements in hospital processes of care that began prior to the prospective payment system have continued after its introduction, but that the likelihood that a patient will be discharged in an unstable condition, has increased. Furthermore, the appropriateness of the DRGclassification as a reimbursement system is questioned: limited information is used in assigning patients to a DRG; the severity of illness and socio-economic characteristics of patients are not considered, and the possibilities for data-manipulation are alarming (Stern 1985, Williams 1984, Simborg 1981, Torf 1989).

In Dutch health care there is no experience with the application of patient classifications systems to finance hospital care. But at the moment political discussions are going on to reorganize the Dutch hospital financing system ('Stelselwijziging Gezondheidszorg'). In the near future, health insurance companies have to come to an agreement with health care institutions about the type, amount and costs of the health care that is provided. Application of a patient classification system may be useful in discussions between health insurance companies and health care providers.

\section{Hospital applications.}

Within a hospital, a patient classification system may have important implications for four types of application.

\section{* internal budgeting.}

Patient classification systems may be helpful in predicting budget requirements, in budget monitoring and in analyzing differences between budget and actual expenditures. Each type of patient requires specific medical care. Determination of the expected costs of medical care for the various types of patients may form a basis for budget allocation for medical departments and subsequently for ancillary departments. A patient classification may be especially helpful in budget analysis. Deviations from the budget may by traced to specific causes such as (a) changes in the number of patients, (b) changes in types of patients (c) changes in resources used within a patient group or (d) changes in costs of the services provided to a paticnt group. 


\section{* planning.}

Historical information about the patient population can help hospitals to develop long-term plans. Expected changes in the patient population may require specific health care facilities. Anticipating changes in patient populations with the help of empirical data may contribute to a timely prediction of the consequences for the hospital.

\section{* utilization review.}

Patient classification systems can be used to examine resource utilization patterns among clinically similar groups of patients and to identify patterns that are atypical. These atypical patterns can then be further evaluated by means of detailed case analysis.

\section{* monitoring the quality of care.}

Physician performance cannot be assessed appropriately without reference to the types of diseases treated. If quality criteria are established for clinically similar groups of patients, deviations from the standard or norm may be observed and singled out for further inquiry.

\section{Research applications.}

Patient classification systems conceming resource use and costs provide detailed data on the types and amounts of health care services used. Such information may be valuable for scientific research in health care in addressing such questions as: What procedures are being performed in various settings, and how often? What types of patients tend to make more use of a particular service? What are the relative costs and outcomes of treating patients in different settings such as teaching versus non-teaching hospitals? Are new services replacing old ones, answering unfulfilled necds, or just duplicating existing services and adding unnecessary costs? (Lichting 1986).

Another application of such patient classification systems may lie in medical technology assessment studies. Classifying patients into clinically relevant categories which are homogeneous with respect to costs may be seen as a precondition for performing these evaluation studies. After grouping, costs and effects of medical care may be determined and compared for each group of patients. Also the effect of changes in medical care (i.e. the introduction of a new technology) may be evaluated in terms of the direct or long-term consequences for the total treatment.

Patient classification systems may thus become a powerful instrument applicable in various areas of health care. During the past decade several researchers have developed methodologies to classify patients and have established applications for these patient classification systems within health care.

The study described in this thesis must be placed in the context of the: ongoing process to find ways to classify patients into clinically relevant categories which are homogeneous with respect to costs. This study concentrates on the development of a method 10 classify patients according to their severity of illness.

In this thesis the following subjects wili be described. First, a theoretical view on patient classification will be given. General criteria for assessing a patient classification system will be listed $(\$ 2.1)$. Well known patient classification systems aimed at predicting or explaining costs of medical care in hospital settings will be described $(\$ 2.2)$. A comparison will be made between characteristics of these systems reported in literature ( $\$ 2.3)$. One of the most well known and widely applied patient classification systems at the moment is the Diagnosis Related Group (DRG)-system. In the Netherlands, and in most other European countries as well as in the US, studies are going on to test the feasibility of this system for various applications within health care. These studies have shown that the DRG-methodology fails 
to incorporate the patient's severity of illness even though severity of illness can be expected to be the most important factor causing differences in costs of medical care.

Subsequently, the research objective of this study will be described. The objective of this study is to investigate the contribution of the patient's severity of illness to explain cost differences between patients in the same diagnosis group ( $\$ 3.1$ and $\S 3.2$ ). Two models will be proposed: one for explaining costs of medical care closely related to the severity condition of a patient during a specific time period in hospital, and another to determine the effect of changes in severity of illness on the cost of medical care during the entire hospital stay $(\$ 3.3$ ). These models will be applied to one specific patient population $(\$ 3.4)$. The research population has been restricted to DRG's comprising patients with Acute Myocardial Infarction (AMI).

Next, the patient population and the data collection performed for this study will be described in chapter 4 . The study involves 464 AMI patients admitted to the University Hospital of Maastricht (the Netherlands) between January 1987 and April 1988.

In chapter 5 a methodology will be proposed to define and operationalize the two most important research concepts. Attention will be paid to the measurement of costs of medical care in hospitals: a methodology will be proposed to allocate costs of ancillary departments to medical care services. Furthermore, a methodology will be proposed to measure the severity of illness of AMI patients.

Subsequently, in chapter 6 , the methodologies proposed will be applied for the research population. Costs of various types of medical care will be determined and described. Furthermore, the measurement of the severity of illness will be described. The severity of several cardiac and cardiovascular deficiencies (coronary stenosis, myocardial ischemia/ necrosis, rhythm and conduction disorders, heart failure and specific complications) will be measured by means of specific clinical indicators. A descriptioñ will also be given of how severity weights derived from a panel of cardiologists will be used to operationalize severity of illness into various severity indices.

In the next chapter the empirical relationship between severity of illness-indicators and costs of medical care will be investigated. The effects of the severity indicators on specific costs will be investigated using regression analysis techniques. The data analysis will be performed separately for: costs of thrombolytic therapy $(\$ 7.2 .1)$, costs of (acute) heart catheterization $(\$ 7.2 .2)$, costs of (acute) PTCA $(\$ 7.2 .3)$, length of hospital stay $(\$ 7.3 .1)$, costs of coronary care unit $(\$ 7.3 .2)$, and other costs of medical care (drugs, laboratory services, electrocardiograms, echocardiography and exercise tests, $\$ 7.3 .3$ ).

The results of the data analysis will be employed to propose a severity of illness classification for AMI patients which on the one hand contains clinicaliy recognizable patient categories that, on the other hand, are homogeneous in an economic sense. Furthermore, an analysis of variance will be made to which degree the new classificiation system and the DRG-system offer explanations for cost differences (chapter 8). Finally, the conclusions from this siudy will be presented in chapter 9 . 


\section{Patient classification systems: a review.}

In this chapter general criteria for developing a patient classification system will be listed ( $\S$ 2.1). Currently used patient classification systems aimed at explaining costs of medical care in hospital settings will be described (\$2.2). A comparison will be made between characteristics of these systems as reported in the literature $(\S 2.3)$.

\subsection{Theoretical background of patient classification systems.}

Each hospitalized patient may be seen as an unique case. Each patient is expected to be treated on an individual basis depending on his/her specific illness condition. However, interpretation of the diagnostic procedures physicians use will eventually result in the definition of the patient's case: the diagnosis labels each patient in a medical sense. The purpose of a patient classification system is not to classify a patient by this label. It aims to classify patients on a more aggregated level by clustering them into patient categories based on similarities between their illness characteristics. Categorizing patients on an aggregated level may produce information which cannot be detected by examining individual cases or labels. Patient classification systems must therefore not be regarded as a tool for describing individual patients but as a tool for describing a hospital population. This enables managers or researchers to understand and analyze medical care processes given to specific iypes of patients.

Although patient classification systems have been applied in various health care studies no comprehensive theory is available indicating how to measure illness characteristics of a patient population. Hornbrook (1982) and Wood (1981) have proposed a methodology to evaluate patient classification systems by specifying general criteria. Each author distinguishes a number of evaluation criteria, of which some are partly overlapping (table 2.1.).

Table 2.1 General criteria for patient classification systems.

$\begin{array}{ll}\text { Hornbook(1982) } & \text { Wood (1981) } \\ \text { * Reliability } & \text { * Medical Meaningfulness } \\ \text { * Validity } & * \text { Economic Meaningfulness } \\ \text { * Sensitivity } & * \text { Administrative Meaningfulness } \\ \text { * Cost-effectiveness } & * \text { Reliability } \\ \text { * Flexibility } & * \text { Practicality } \\ \text { * Acceptability } & * \text { Versatility }\end{array}$

The most important criteria mentioned by both authors are the medical meaningfulness and the economic meaningfulness of a patient classification system. 


\section{Medical Meaningfulness/Content Validity.}

Both authors explicitly mention that a patient classification system must contain medically relevant patient categories. Medical meaningfulness is defined by Wood as 'the extent to which knowledge of a patient's case type alone - without other information about the individual patient - conveys clinical expectations and enables clinicians to exchange information about those expectations. For a group of clinicians, a medically meaningful classification stimulates expectations as to the natural history of the disease, the appropriate ways to manage the case, the prognosis, the likelihood of complications and the risk of death'. Hornbrook describes this requirement more generally as the content validity of a system and defines it as 'the representativeness and comprehensiveness of the content of the measuring instrument. For patient classification systems it refers to the breadth of coverage of the various case types and the dimensions of the patient population relevant for the purpose'.

\section{Economic Meaningfulness/Predictive Validity.}

The other important requirement for patient classification systems aimed at analyzing costs of medical care is the economic meaningfulness of the patient categories. Wood says that "a classification is economically meaningful if, within its case types, the vectors of amounts of the various goods and services needed for the patient's clinical management are homogeneous. This means that the patients in any case type use about the same array of goods and services and that the required amount of any particular good or service is fairly constant from patient to patient'. Hornbrook describes this as predictive validity which he defines as 'the ability of the measure to predict some outcome or event that is hypothesized to be related to the fundamental concepts. Thus, a valid resource-intensity case-mix measure will predict total hospital costs; a valid length of stay case-mix measure will predict overall length of stay; and a valid mortality casc-mix measurc will predict overall death rates.'

Other criteria mentioned by both authors are the sensitivity and reliability of a classification system. Sensitivity, as described by Hombrook refers to the ability of a case-mix measure to discriminate adequately among hospitals with respect to the relevant dimension(s) of their outputs. How small a difference in the case mix can the index detect?' Reliability as described by Hornbrook refers to "the dependability, stability, consistency, predictability and accuracy of the measurement system. If a measure is reliable, repeated applications of the measure to the same set of hospitals provide the same or similar results'. According to Wood, a classification is reliable if any patient is placed in the same case type no matter who or what mechanism is entrusted with the assignment. Two qualities influence classification reliability: manipulation (of data or by management) and precision (data (quality)'.

Other requirements as described by the authors emphasize more pragmatic issues. According 10 Wood a classification must be administratively meaningful if it is to be used in hospital planning or administration. Wood points out the possibility of using the classification for internal hospital management. The measure has to correspond with the way the hospital is organized and has to fit in with other already existing procedures in measurement. Hormbrook emphasizes this by using the term acceptability: the users must believe in the usefulness of the measurement system for policy or administrative purposes. Wood also suggests that a patient classification must be versatile. He suggests that "its categories can be put together in different ways to form different uses. specifically classifications for review of medical care, for administrative control, and for prospective reimbursement.' Hornbrook describes this as the nexibility of a system. referring to the property of a measure to be used for multiple purposes. 
Hornbook also mentions the cost-effectiveness of a patient classification system. This criterium relates to 'selecting the least cost method of measurement, without adversely affecting any of the other properties of the measure'. Wood refers to this as practicality. Both emphasize the conscious decision a hospital has to make in this matter with respect to investments and expected benefits in the long run.

The criteria as discussed by both authors can be seen as general guidelines according to which a classification system can be evaluated. However, from these general criteria no fundamental and comprehensive theory for patient classification systems can be derived. According to Wood a complete theory should suggest various weighting siructures and techniques for mathematically combining cases. Both authors do not make clear how to distinguish or prioritize their general criteria. It is clear that all criteria can not be fulfilled in an optimal way within one and the same classification system. Nor do they present ways of operationalizing the criteria. The medical meaningfulness (or content validity) is, for example, an issue which is from a methodological point difficult to attain. Clinical recognizability may vary from one physician to another. In addition, the principles of economic and medical criteria can be counteractive to each other: a patient classification that is relevant to clinicians need not be economically homogeneous, or a patient grouping used by economists may be an unrecognizable amalgamation to physicians. Furthermore, the relative importance of each criterion depends on the specific purpose of a classification system. A system developed to predict death rates will tend to be less relevant to predict hospital costs.

In spite of the lack of a complete theory about patient classification systems one may conclude that at least these general criteria have to be fulfilled as much as possible. Further conceptualisation in this field is certainly necessary and may contribute to the development of theories on patient classification systems.

In recent years attempts have been made to develop patient classification systems that may be useful in analyzing medical care processes; these systems will be deseribed in the next paragraph.

\subsection{Review of patient classification systems.}

In this paragraph a number of patient classification, systems that have been developed during the past decade will be reviewed. Only those systems have been selected that are frequently used in analyzing costs of medical care, or that are likely to become widely used systems. Furthermore, only those systems are described which classify the greater part of the clinical hospital population. The systems under review are listed in table 2.2 . The systems will be briefly described in terms of conceptualization, classification method and classifying variables. For each classification system the criteria used to classify patients with Acute Myocardial Infarction will be given. In $\$ 2.3$. their medical meaningfulness/content validity and their economic meaningfulness/predictive validity will be reviewed and evaluated. 
Table 2.2. Review of Patient Classification Systems during last decade.

Patient Chssification System

Diagnosis Related Groups (DRG)

Patient Management Categories (PMC)

Disease Staging

Computerized Severity of Illness (CSI)

Acute Physiology and Chronic Health

Evaluation (APACHE)

Medical Illness Grouping System

(MEDISGRPS)
Researcher(s)

Fetter, Freeman, Nederstigt

Young

Gonella

Horn

Wagner, Draper

Brewster

\subsubsection{Diagnosis Related Groups.}

The DRG-methodology was developed by Fetter e.a. (1980) at Yale University. Originally, the Diagnosis Related Groups (DRG)-system was developed for utilization review, but since 1982 it has been used as a prospective financing system for Medicare patients in the U.S.A..

One of the most important criteria used when developing the DRG-system was that each patient category had to be medically interpretable. The researchers did make use of the Delphi method with pancls of physicians. The panels reached consensus on categories expected to be medically recognizable. Another important criterion was that (given the content validity of the categories) each group was expected to have a statistically narrow range in the distribution of length of stay and costs. Analysis of variance was applied to test the predictive validity of the length of stay by the system.

In addition some pragmatic criteria were applied: there must be a manageable number of groups which are mutually exclusive. the definitions of the group must be exhaustive and the groups must have sufficient numbers of patients. Furthermore, classes of patients are defined in terms of variables normally collected and available in hospital medical abstract records, i.e. computerized discharge data including amongst others diagnoses and surgical procedures as defined by the International Classification of Diseases, 9-th revision (the ICD-9-cm coding scheme).

The researchers ended up, in 1981, with a patient classification system containing 467 catcgories. The classification process is briefly explained by Averill e.a. (1986) in the DRGDefinition Manual: 'The process of forming the DRG's was begun by dividing all possible principal diagnoses into 23 Major Diagnostic Categories (MDC's). The 23 MDC's were formed by panels of physicians as the first step toward ensuring that the DRG would be clinically coherent. The diagnoses for each MDC correspond to a single organ system or eliology. and are in general associated with a particular medical specialty. Once the MDC's were defined each MDC was evaluated to identify those additional patient characteristics which would have a consistent effect on the consumption of hospital resources. Since the presence of a surgical procedure which required the use of the operating room would have a significant effect on the type of hospital resources used by a patient, most MDC's were initially divided into medical and surgical groups. Then, in general, the surgical patients were further defined based on the precise surgical procedure performed while the medical patients were further defined based on the precise principal diagnosis for which they were admitted to the hospital. Since a patient can have multiple procedures related to their principal diagnosis during a particular hospital stay, and a patient can be assigned to only one surgical class, the surgical classes in each $M D C$ were defined in a hierarchical order. 
Patients with multiple procedures would be assigned to the surgical class highest in the hierarchy.

Once the medical and surgical classes for an MDC were formed, each class of patients was evaluated to determine if complications, co-morbidities or the patient's age would consistently affect the consumption of hospital resources. The final variable used in the definition of the DRG's was the patient discharge status. The actual process of forming the DRG's was highly reiterative, involving a combination of statistics results from test data with clinical judgment'.

An example of the classification process for non-surgical patients with Acute Myocardial Infarction is outlined in figure 2.3. The DRG-system distinguishes three DRG's for these patients. A list containing specific cardiovascular diagnoses is used to separate complicated from non-complicated patients. Furthermore, patients who die during hospital stay are grouped into a separate DRG.

Figure 2.3. DRG-Classification of patients with Acute Myocardial Infarction.

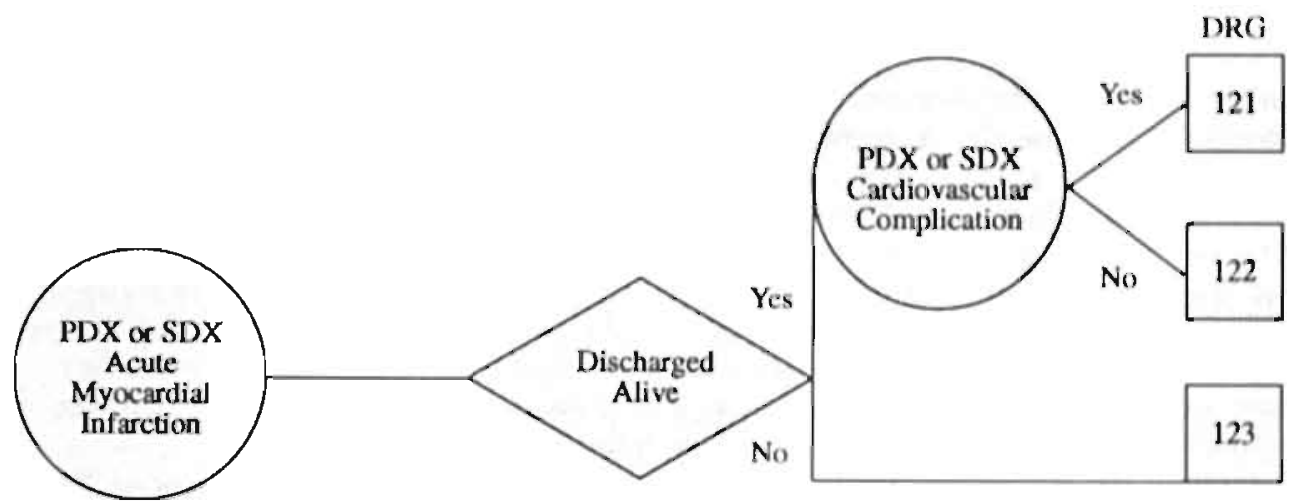

PDX $=$ primary diagnosis

Source: Health Systems International, 1986

SDX $=$ secondary diagnoșis

During a period of more than 10 years (1977 -1991) the DRG-system was subjected to modifications (Mc Guire 1990, Health Information Systems 1991). Recently the researchers have proposed the 'Refined DRG-system' in which the severity of illness is more specifically included. Surgical and diagnostic categories (Adjacent DRG's) have been subdivided by sets of specific complications and comorbidities (Health Systems Management Group, 1990).

Because of the expected differences in the organization of American and Dutch health care, a feasibility study for applying DRG's in Dutch health care was carried out (Nederstigt, 1985). Nederstigt recommends that only the American DRGi method must be applied, but that specific Dutch DRG-definitions need to be developed. In that way one will get a patient classification system that is expected to have more medical relevancy to Dutch clinicians.

The feasibility study took place at three general hospitals in the Netherlands. Most of the criteria and guidelines used by Fetter e.a. were not changed, except that in the Dutch DRGclassification a hierarchy in diagnostic categories has also been applied. The variance explained by the Dutch DRG-system concerning the length of stay turned out to be similar to that of the American DRG-system. Per MDC the variance explained varied between $5 \%$ and $70 \%$. The variance explained in length of stay for surgical DRG's was higher than for 
non-surgical categories. Differences in types of treatment within ambulatory care and clinical care did account for some improvements in medical meaningfulness: the same type of treatment performed as clinical care in the Netherlands may have been performed as ambulatory care in the U.S. In addition, differences in the definition of pediatric care caused some changes. Instead of using the criterion 'age 18 years and older', the Dutch DRG version, reduced the age criterion to ' 15 years and older'. Some changes occurred as a result of differences in medical definitions. Particularly in surgical categories changes such as creating specific new surgical categories or re-allocating operating room procedures within the existing categories have taken place. In addition, changes were made in the hierarchy of the surgical categories. In the diagnostic categories specific secondary diagnoses were used to define the ultimate DRG's (Fetter e.a. also refined the DRG-system on the basis of specific secondary diagnoses). Although the elinical relevance did increase, much heterogencity in length of stay within the Dutch diagnostic DRG's still existed. Nederstigt (1985) presumes that this is because the DRG-system does not specifically take into account the patient's severity of illness.

\subsubsection{Patient Management Categories.}

Patient Management Categories (PMC's) is a patient classification system developed by W. Young e.a. at Blue Cross of Western Pennsylvania. FMC's were designed to identify clinically specific patient types, each requiring a distinct diagnostic and treatment strategy. The original development of PMC's (Young. 1982) was not only based on medical discharge data, but also on the reasons for hospital admission. According to Young the principal discharge diagnosis alone, even if it is accurately identified, cannot reflect what happened during the patient's hospital stay. Adding other data, such as secondary or multiple diagnoses and procedures, will not solve the problem. Patient categorization in terms of all these variables at discharge may result in some sort of clinical similarity at discharge but dissimilarity with respect to the medical care during hospital stay.

Integrating the admission condition into a classification scheme is, according to Young. necessary to define groups of patients with similar medical care patterns. For each type of patient, the typical diagnostic and treatment strategies applied and the resources generally used within each hospital stay can then be identified.

In summary:

1. the PMC"s were developed using admission state and discharge diagnosis;

2. the PMC's were firsi defined by physicians and then applied in data analysis;

3. for every PMC. Patient Management Paths are developed which consist of diagnostic and treatment services for effective care for the typical patient in each PMC.

However, recent developments in the PMC-system have caused some important changes in the methodology. Because the reason for admission is not always computerized and commonly available in hospitals, the recently computerized PMC-version only uses dischatge data (The Pittsburgh Research Institute, 1988). It distinguishes about 800 different categories of which approximately 300 PMC's classify approximately 80 \% of a hospital population.

The PMC-assignment process (Young, 1986) is based on principal and secondary diagnoses and surgical procedures as coded according to the ICD-9-cm code at the patient's discharge from hospital. The PMC methodology takes into account the fact that several interrelated diagnoses often represent one manifestation of a single disease, and do not necessarily reflect disease complications or co-morbidities. For this reason, combinations of diagnoses are used to assign a patient to a PMC. The sequence of diagnoses listed on the discharge abstract is disregarded. A patient may be assigned to more than one PMC if unrelated co- 
morbid conditions are present. Eventually, only a single PMC will be assigned. In order to determine which PMC should be selected, an order of relative severity and/or difficulty of management was developed. This allows the assignment of a patient to a single PMC with the highest severity ranking.

As an example the PMC classification for surgical and non-surgical patients with acute myocardial infarction is presented in table 2.4. This system distinguishes eight different categories of AMI patients while using specific secondary diagnoses, diagnostic tests and surgical procedures in the classification process.

\section{Table 2.4. Patient Management Categories for patients with acute myocardial infarction.}

$\begin{array}{ll}0301 & \text { AMI: ACUTE MYOCARDIALINFARCTION } \\ 0302 & \text { AMI: TACHYRHYTHMIA } \\ 0303 & \text { AMI: BRADYRHYTHMIAHEART BLOCK } \\ 0304 & \text { AMI: HYPERTENSION } \\ 0306 & \text { AMI: CONGESTIVE HEART FAILURE WOPERATION } \\ 0307 & \text { AMI: CONGESTIVE HEART FAILURE WIO OPERATION } \\ 0308 & \text { AMI: CARDIOGENIC SHOCK } \\ 0309 & \text { AMI: CARDIAC CATHETERIZATION/PTCA }\end{array}$

Source: The Pittsburgh Research Instimte, 1988

\subsubsection{Disease Staging.}

Disease Staging was developed by Gonella e.a. (1984) for measuring disease severity. Severity is defined by Gonella as the likelihood of death or residual impairment as the result of a disease, without consideration of treatment. The idca behind 'Staging' is borrowed from clinical oncology, in which various stages in the course of diseases can be defined. Gonclla applied this concept to other medical and surgical problems in order to classify all hospitalized patients. The development of staging criteria was limited to the major diseases in each etiology-body system class.

According to Gonella (1984) diseases should be distinguished from health care problems and conditions. Classifying patients on the basis of conditions and health problems without considering the underlying causes is not justified. Approximately 420 diseases are identified in the Staging process. The diseases are characterized by four primary categorics of increasing levels of severity:

stage 1: conditions with no complications or problems of minimal severity;

stage 2: problems limited to an organ or system, with a significantly increased risk of complications over stage I;

stage 3: multiple site involvement, generalized systemic involvement, poor prognosis; stage 4: death.

Panels of medical consultants specified the medical staging criteria for the primary stages. and, if necessary, the substages within every primary stage category. Disease Staging pertains to two related approaches for measuring severity:

(1) Clinical Staging, a method based on a manual review of clinical measurements documented in the medical record. For each of the 400 disease categories clinical criteria have been developed indicating values (laboratory, radiographic findings, vital signs, etc.) which characterize the stages and which represent the severity icvels within the disease. 
(2) Coded Staging, an automated method based on the coded diagnostic data contained in the discharge abstract. Each of the criteria defined in the clinical staging method were converted into ICD-9-CM codes. Because of the lack of computerized data in most discharge abstracts such as laboratory findings. changes have been made in the medical criteria of the Coded Staging System.

The staging concept emphasizes the medical relevancy of the criteria. In patients with multiple non-related diseases the overall severity is captured by the highest stage level of only one disease. One important condition for applying the Disease Staging method is that the abstract data must be complete; when one or more secondary diagnoses are missing the severity can be underestimated. In the development of the Disease Staging mechanism no statistical or economic analysis was applied. However, using this classification system to analyze resource data has demonstrated that there is a relationship between Staging categories and costs (Conklin 1984, Garg 1978, Louis 1984).

The criteria for clinical and coded Disease Siaging of patients with Acute Myocardial Infarction are presenied in table 2.5. Four primary stages including 10 substages of the course of the illness of AMI patients have been distinguished.

Table 2.5. Staging criteria in Clinical and Coded Disease Staging for patients with Acute Myocardial Infarction.

\begin{tabular}{|c|c|c|}
\hline STAGH & $\begin{array}{c}\text { COMMON DESCRIPTION } \\
\text { OR } \\
\text { NAME OF THE CONDITION }\end{array}$ & SUPPORTING EVIDENCE OR CLUES \\
\hline 1.1 & 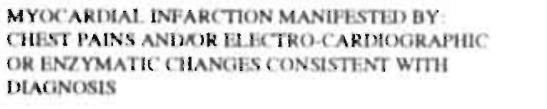 & $\begin{array}{l}\text { HISTORY, PHYSICAL EXAM. ABNORMAL ENZYMES } \\
\text { AND EKG } \\
\text { CHANGES CONSISTENT WTH MI (SEE REFERENGE } \\
\text { MATERLAL) }\end{array}$ \\
\hline 12 & 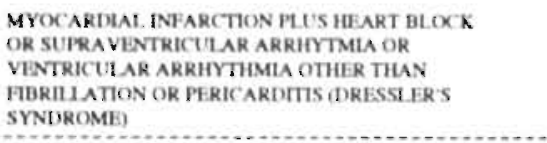 & \\
\hline 21 & $\begin{array}{l}\text { MYOCARDAL. INFARCTION PLUS EMBOLITO } \\
\text { OTHER ORGAN SYSTEMS LFADING TO } \\
\text { A FIRIFHERAL YASCULAR OCCLUSION } \\
\text { OR INFARCTIONOF GI TRACT OR INFARCTION } \\
\text { OF KIDNEY }\end{array}$ & $\begin{array}{l}\text { PHYSICAL FINDINGS AND ABNORMAL. LABORATORY } \\
\text { FINDINGS }\end{array}$ \\
\hline 22 & 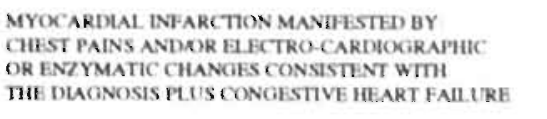 & \\
\hline 21 & CERHBRAL VASCULAR ACCIDENT (CVA) & \\
\hline 34 & PAPILLARY MUISCTE RITTURE & \\
\hline 25 & VENTRICULAR ANEURYSM & \\
\hline 31 & 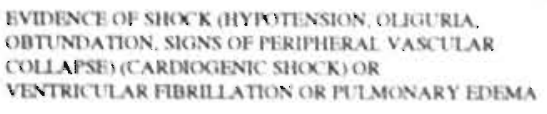 & $\begin{array}{l}\text { FHYSICAL, FINDINGS AND ABNORMAL. LABORATORY } \\
\text { FNDINGS }\end{array}$ \\
\hline 32 & CARDLAC ARREST & $\begin{array}{l}\text { SECONDARY TO MYYOCARDLAL: INFARCTION } \\
\text { WITH SICCESFII. RESTSCTIATION }\end{array}$ \\
\hline 40 & DEATH & \\
\hline
\end{tabular}




\subsubsection{Computerized Severity of Illness.}

The Computerized Severity of Iliness-system was developed by Horn c.a. (1986) Io measure the hospital case mix in relation to the patient's total burden of illness. The Computerized Severity of Illness (CSI) is determined by clinical criteria such as results (rom physical examination and various diagnostic findings. The CSI-system, modeled after the Original Geverity of Illness. (Hom, 1983,1985), was developed in order to facilitate widespread colkection of sewerity of illness data.

The CSI classification uses objective signs and symptoms, laboratory values, radiology findings etc , commonty available in the medical record, to assign a patient into one of four severily levels. The severity indicators are derived from discussions with medical consultans, in which morbidity and hemhood of death are used as guiding criteria.

For every $1 \mathrm{CD}-9$-cm code, specific sets of chinical criteria have been developed and put into a matrix, representing a gradation in severity. Several 1 CD-9-cm diagnostic codes can refer to one severity matrix. Altogether, more than 700 separate criteria sets, corresponding 10 over 10.000 icD-9-Cm codes, have been developed. The CS1 score can he expressed by an Admission-score (based on diagnostic tindings on admission) and a Maximum-score (throughout the entire hospital stay). An example of a severity matrix for patients with the diagnosis Acute Myocardial Infarction is presented in table 2.6. If at least two crieriat within a severity level (column) are present the patient win get the score belonging to this column. If one or more diseases are present during the hospital stay. the severty score is estublisted for each separate disease. computer algothm with weighting nies, aking into accoun the interactive effects of the condituns, can be applied to nroduce an overall Computerized Severity of lliness index. During the devetopment of the CSI no economic analysis was performed; the severity criteria and their corresponding severity level were derived from lengthy sessions with physicians. Use of this classification system to analyze resource data has demonstrated that there is a relationship between the CSI-score(s) and costs (Hom 1986, Horn 1991).

\subsubsection{Acute Physiology And Chronic Health Livaluation.}

According to its developers (Wagner e.a., 1984) APACHE II (Acute Physiology and Chronic Health Evaluation, second version) is a severity of illness classification that employs basic physiologic measures to stratify patients according to risk of death, independent of the diagnostic category assigned by physicians. Their original goal was to describe and evaluate intensive care unit (ICU)-patients. Because the system is not diagnosis-specific they clamed it could be also be used for other patient populations. APACHE II classifies patients into severity categories based on diagnostic data, collected early in the course of cach hospital stay (within 24 hours of ICU admission), rather than during or after hospital discharge. The researchers assume that the patient's severity on admission will reflect the burden of illness while in hospital, in the most valid way. 
Table 2.6. Computerized severity of illness criteria for patients with Acute Myocardial Infarction.

\begin{tabular}{|c|c|c|c|c|}
\hline CATEGORY & $!$ & 2 & 3 & 4 \\
\hline $\begin{array}{l}\text { CARDIO. } \\
\text { VASCULAR }\end{array}$ & $\begin{array}{l}\text { - EKG changes- } \\
\text { ST segment } \\
\text { changes } \\
\text { - C. } \\
>=2.84\end{array}$ & $\begin{array}{l}\text { - }>6 \text { PVCsimin } \\
\\
\text { - muffled heart } \\
\text { sounds } \\
\text { - C.O. } \\
2.7 .2 .3 \mathrm{~L}\end{array}$ & $\begin{array}{l}\text { - third degree } \\
\text { beart block } \\
\\
\text { - C.O. } \\
2.2-1.91\end{array}$ & $\begin{array}{l}\text { * life- } \\
\text { threatening } \\
\text { arrbythmias } \\
\text { and/or } \\
\text { hypotension } \\
\\
\text { - C. } \\
<=1.8 \mathrm{~L}\end{array}$ \\
\hline FEVER & & $\begin{array}{l}->=100.5 \text { and } \\
\text { or rigors }\end{array}$ & & \\
\hline $\begin{array}{l}\text { GENITO- } \\
\text { URINARY }\end{array}$ & & & $\begin{array}{l}\text { - U.O. } \\
\text { 499-100ce. }\end{array}$ & $\begin{array}{l}\text { U.O. } \\
c=99 \mathrm{ce}\end{array}$ \\
\hline $\begin{array}{l}\text { LABS } \\
\text { CIIIMISTRY } \\
\text { HEMATOLOKFY }\end{array}$ & $\begin{array}{l}\text { - CPK } \\
<3.0 \times \text { norm } \\
\text { - WBC } \times 1000 \\
45.11 .0 \\
\text { icu mm. } \\
\text { Bands } \\
<10 \% \\
\text { SED. RATE } \\
<=49 \mathrm{~mm} / \mathrm{hr}\end{array}$ & $\begin{array}{l}\text { - CPK } \\
3.0-49 \times \text { norm } \\
\text { WBC } \times 10 \times 0 \\
11.1 .20 .0 \\
/ \mathrm{cu} \text { mm: } \\
\text { Bands } \\
10-20 \% \\
\text { SED RATE: } \\
>=50 \mathrm{~mm} / \mathrm{hr}\end{array}$ & $\begin{array}{l}\text { - CPK } \\
5.0-9.9 . \times \text { norm } \\
\text { - WBC } \times 1000 \\
20.1-30.0 \\
\text { /cu cnm; } \\
\text { Bands } \\
21-40 \%\end{array}$ & $\begin{array}{l}\text { CPK } \\
>=10.0 \times \text { norm } \\
\text { WBC } \times 1000 \\
>=30.1 \\
\text { lcu mm: } \\
\text { Bands } \\
>40 \%\end{array}$ \\
\hline NEUROLOGY & & & - confusion & - unresponsive \\
\hline PAIN & $\begin{array}{l}\text { chest pain } \\
c=15 \mathrm{~min}\end{array}$ & $\begin{array}{l}\text { - chest pain } \\
\text { at rest }\end{array}$ & & \\
\hline $\begin{array}{l}\text { RAIJIOLOOGY } \\
\text { CIIIEST }\end{array}$ & $\begin{array}{l}\text { - normal } \\
\text { cardiothoracic } \\
\text { ratio }\end{array}$ & $\begin{array}{l}\text { - cardiothoracic } \\
\text { ratio } 50-54 \%\end{array}$ & $\begin{array}{l}\text { cardiothoracic } \\
\text { ratio } 55.59 \% \text {; } \\
\text { moderate } \\
\text { pulmonary } \\
\text { vascular } \\
\text { congestion }\end{array}$ & $\begin{array}{l}\text { cardiothoracic } \\
\text { ratio }>=60 \% \text {; } \\
\text { pulmonary } \\
\text { edema }\end{array}$ \\
\hline $\begin{array}{l}\text { RESPIRA- } \\
\text { TORY }\end{array}$ & & $\begin{array}{l}\text { - dyspnea on } \\
\text { exertion }\end{array}$ & $\begin{array}{l}\text { - peripheral } \\
\text { cyanosis } \\
\text { - dyspnea at } \\
\text { rest }\end{array}$ & $\begin{array}{l}\text { central } \\
\text { cyanosis } \\
\text { - apnea. }\end{array}$ \\
\hline SKIN & - diaphoretic & & & \\
\hline
\end{tabular}

Source: Health System International, 1987

Clinical criteria include: heart rate, blood pressure, temperature, respiratory rate, the Glasgow Coma Score, five variables obtained from routine blood samples and two variables obtained from arterial blood tests. The criteria were selected by physicians with regard to validity and specificity of measures, breadth of vital organ system coverage, objectivity, reliability and frequency of measurement. The scores on the 12 physiologic variables are added to form an Acute Physiology Score (APS), described in table 2.7. The full APACHE 11 score for ICU patients consists of the sum of points for APS. plus weights assigned for age category and for severely disabling chronic diseases. A patient can maximally be assigned to a total score of seventy-one points; relatively high scores indicate a high severity. APACHE II was developed as a means of predicting death or survival during hospital stay, but data analysis has also shown a relationship between the scores of APACHE 1I. total costs of intensive care episodes and total length of hospital stay (Wagner, 1984). 
Table 2.7. Criteria for grouping patients, severity of illness according to APACHE II.

THE ACUTE PAYSIOLOOY AND CHRONOC HENTH EVALUATON (APACHE) II SEVERTYY OF DISEASE CLASSHACATION SYSTEM

\begin{tabular}{|c|c|c|c|c|c|c|c|c|c|}
\hline \multirow{3}{*}{ BHYSIOLOCIC VARLABELE } & \\
\hline & \multicolumn{6}{|c|}{ high aboormal nage } & \multicolumn{3}{|c|}{ low abeormal raspe } \\
\hline & .4 & $\cdot 3$ & +2 & +1 & 0 & +1 & $\bullet 2$ & .3 & +4 \\
\hline temperature - rectal $\left(\mathrm{C}^{-}\right)$ & $241^{*}$ & $39^{\circ}-40.9^{*}$ & & $38.5-38.9+$ & $36=-38.4^{*}$ & $34^{*} \cdot 35.9 *$ & $32^{*}, 33.9^{*}$ & $\mathbf{3}+31.9$ & $\leq 20.0^{\circ}$ \\
\hline mean arterial pressure - mm $\mathrm{Hg}$ & 2160 & $130-159$ & $110-129$ & & $70-109$ & & $50-69$ & & $\$ 49$ \\
\hline beart rate (veatricular reapotase) & 2180 & $140-179$ & $110-139$ & & $70 \cdot 109$ & & $55-69$ & 40.54 & $\leq 39$ \\
\hline \multicolumn{10}{|l|}{ respiratory rate - (nov veatilated } \\
\hline of ventilated) & $\geq 50$ & 35.49 & & 25.34 & $12 \cdot 24$ & $10-11$ & $6-9$ & & $\$ s$ \\
\hline \multicolumn{10}{|l|}{ orygenation $\mathrm{A} \mathrm{aDO}_{2}$, or $\mathrm{PaO}_{2}\left(\mathrm{~mm} \mathrm{H} \mathrm{H}_{8}\right)$} \\
\hline$=\mathrm{FiO}_{2} \geq 0.5$ record $\mathrm{A}$ aDOH & 2500 & $350-499$ & $200-349$ & & $<200$ & & & & \\
\hline $\mathrm{bFO}_{2} \geq 0.5$ record only $\mathrm{PMO}_{2}$ & & & & & $\mathrm{MO}_{2}>70$ & $\mathrm{PO}_{2} 6 \mathrm{i}-70$ & & $\mathrm{mo}_{2} 55 \cdot 60$ & $\mathrm{PO}_{2}<33$ \\
\hline anterial pH & 27.7 & $7.6-7.69$ & 7.6 .69 & 75.7 .59 & 733.7 .49 & & 7.25 .732 & 7.15 .7 .24 & $<715$ \\
\hline serum sodium imMollt.) & 2180 & $160-179$ & $155-159$ & $150-154$ & $130-140$ & & $120-129$ & $111 \cdot 119$ & 5110 \\
\hline serum potassium (mMol/L) & 27 & $6-6.9$ & & 5.5 .5 .9 & 3.5 .5 .4 & 3.3 .4 & 2.5 .2 .9 & & $<25:$ \\
\hline \multicolumn{10}{|l|}{ serum creatinine (me/ $100 \mathrm{ml}$ ) } \\
\hline (double point score for acute tenal failure) & 235 & $2 \cdot 3.4$ & 1.5 .1 .9 & & $0.6-1.4$ & & $<0.6$ & & \\
\hline bematiogrit & 260 & & $50-599$ & 46.49 .9 & 30.45 .9 & & $20-290$ & & $<20$ \\
\hline white blood count (total/mm $\mathrm{mm}^{3}$ ) (in 1.000s). & 240 & & $20-39.9$ & $15-19.9$ & $3-149$ & & 1.29 & & $<1$ \\
\hline \multicolumn{10}{|l|}{$\begin{array}{l}\text { Gimagow corra score (CCS) } \\
\text { core }=15 \text { minus actual GCS }\end{array}$} \\
\hline \multicolumn{10}{|l|}{$\begin{array}{l}\text { A total acute physiology score (APS) } \\
\text { wim of the } 12 \text { individual vanable points }\end{array}$} \\
\hline (act preferred, use if Do ABGs) & $2 \$ 2$ & $4 \mathrm{~T}-51.9$ & & $32-40.9$ & $22 \cdot 3 r 9$ & & 18.21 .9 & 15.170 & $<15$ \\
\hline \multirow{2}{*}{\multicolumn{2}{|c|}{$\begin{array}{l}\text { B AGE POINTS } \\
\text { Aasign poiats to age as follows }\end{array}$}} & \multicolumn{5}{|c|}{ C CHRONICAL HEALTH POINTS } & \multicolumn{3}{|c|}{ APACHE IS SCOKE. } \\
\hline & \multirow{2}{*}{$\begin{array}{l}\text { Asaign points to age as follows } \\
\text { Age (mn) } \quad \text { Points }\end{array}$} & \multicolumn{5}{|c|}{ If the patiest has a history of severe organ } & \multicolumn{3}{|c|}{ Sum of $A, B \operatorname{esc}$} \\
\hline Age (mn) Pounts & & \multicolumn{5}{|c|}{ system inaufficiency of is immuno } & A APS pour & & \\
\hline$\$ 44$ & & comprom & 4 assign poit & as follows & & & B Age poit & & \\
\hline $45-54$ & & a. for noo & crative of en & gescy & & & C Orronic: & atth points & \\
\hline $55-64$ & & pontoperat & e patjeats - 5 & ints & & & Tout APA & IE II & \\
\hline $65-74$ & & of & & & & & & & \\
\hline 275 & & b. for elec & e postoperat & parieats -2 po & & & & & \\
\hline
\end{tabular}

Source: Wagner, 1984

\subsubsection{The Medical Illness Severity Grouping System.}

The Medical Illness Severity Grouping System (MEDISGR.PS) is also an admission oriented severity grouping system developed by Brewster e.a. (1985). MEDISGRPS classifies hospital patients into one of five admission scverity groups. It measures changes in scverity over the course of the inpatient stay and monitors the results of the care provided and the resources consumed. Assignment depends on the reason for admission and the patient's Key Clinical Findings (KCF's), which are assumed to be objective indicators of an abnormai condition. K.CF's include laboratory, radiology, pathology and physical examination resuits, Approximately $500 \mathrm{KCF}$ 's are distinguished in this system. The KCF's were derived by 
screening the illness information transfer between the residents at morning call and the residents coming on duty. It is assumed that during this process, information exchange is focused on relevant key clinical findings in newly admitted patients. Each KCF is assigned to a severity level based on the clinical perception of the potential degree of organ failure. The most severe KCF determines the patient's severity category. If two or more KCF's occur at the same severity level, the patient is placed into the next highest severity category, because the outcome and resource use of these patients is assumed to be the same as that of patients at the higher severity level.

A patient may be categorized into one of ive severity categories:

Severity group 0: patients with no KCF's;

Severity group 1: patients with 'minimal findings' indicating a low potential for organ failure;

Severity group 2: patients with either acute findings connoting a shor time course, with an unclear potential for organ failure, or severe findings with potential for future organ failure;

Severity group 3: patients with both acute and severe findings indicating a high potential for imminent organ failure

Severity group 4: patients with critical findings indicating the presence of organ failure.

Severity group 5: death.

An example of the MEDISGRPS classification for patients with acute myocardial infarction is presented in table 2.8. Like APACHE II, MEDISGRPS is constructed independently of the clinical diagnosis of physicians. According to Brewster the KCF's are the functional manifestation of the illness and therefore data collection and severity classification are independent of the final discharge diagnosis. MEDISGRPS therefore provides an indication of how ill a patient is relative to all other patients, irespective of the patient's diagnosis.

Table 2.8. Medisgrps criteria for patients with acute myocardial infarction.

\begin{tabular}{|c|c|c|c|c|}
\hline \multirow{2}{*}{$\begin{array}{l}\mathrm{KCF} \\
\text { categorie }\end{array}$} & \multicolumn{4}{|c|}{ severity group } \\
\hline & 1 & 2 & 3 & 4 \\
\hline I:KCi & $\begin{array}{l}\text { Ischemia } \\
\text { Airial } \\
\text { fibrillation }\end{array}$ & $\begin{array}{l}\text { Myocardial } \\
\text { infarction } \\
\text { (acute) } \\
\text { (extension) }\end{array}$ & $\begin{array}{l}\text { 3rd-degiee } \\
\text { heart biock }\end{array}$ & \\
\hline Chest $x$ ray & Cardiomegaly & & $\begin{array}{l}\text { Congestive } \\
\text { heart failure }\end{array}$ & \\
\hline $\begin{array}{l}\text { Physical exam } \\
\text { Cardial cathe- } \\
\text { terization }\end{array}$ & Rales & $\begin{array}{l}\text { Cardiomyo- } \\
\text { pathy }\end{array}$ & & Coma \\
\hline Laboratory & $\begin{array}{l}\mathrm{PO}_{2} 60-69 \\
\mathrm{CPK} 121-239\end{array}$ & $\begin{array}{l}\mathrm{PO}_{2} 45-59 \\
\mathrm{CPK} 2404\end{array}$ & $\mathrm{PO}_{2}<45$ & \\
\hline Vital signs & $\begin{array}{l}\text { Respirations } \\
25-32\end{array}$ & $\begin{array}{l}\text { Respirations } \\
>32\end{array}$ & & \\
\hline
\end{tabular}




\subsection{Evaluative comparison of patient classification systems.}

The patient classification systems presented in the previous paragraphs show a variety of ways to measure characteristics of a hospital population. The systems differ in goal, conceptualization, classification variables, classification method, moment of measurement during stay, number of patient categories, etc.. Because of these differences in goals and conceptual differences, results and applications may also be expected to be different. All authors have in common that they want to provide an instrument to measure hospital population characteristics by means of disease-related variables or indicators in order to analyze complex hospital care processes. A summary of goals and characteristics of the patient classification systems under review is presented in table 2.9. In this paragraph an attempt will be made to compare the medical meaningfulness (the content validity), the economic meaningfulness (the predictive validity), the reliability and the practicality of these systems.

Table 2.9. Summarized characterization of six patient classification systems.

\begin{tabular}{|c|c|c|c|c|}
\hline $\begin{array}{l}\text { Patient classifica- } \\
\text { tion system }\end{array}$ & Original purpose & Source & $\begin{array}{l}\text { Classification } \\
\text { variables }\end{array}$ & $\begin{array}{l}\text { Number of } \\
\text { parient care- } \\
\text { gories }\end{array}$ \\
\hline$D R G$ & $\begin{array}{l}\text { Utilization } \\
\text { Review }\end{array}$ & $\begin{array}{l}\text { Discharge } \\
\text { Abstract }\end{array}$ & $\begin{array}{l}\text { Diagnoses } \\
\text { Surgical Procedures } \\
\text { Age } \\
\text { Sex } \\
\text { Discharge situation }\end{array}$ & $467^{*}$ \\
\hline$P M C$ & $\begin{array}{l}\text { Financing } \\
\text { Abstract }\end{array}$ & Discharge & $\begin{array}{l}\text { Diagnoses } \\
\text { Surgical Procedures } \\
\text { Age } \\
\text { Sex }\end{array}$ & $>800$ \\
\hline $\begin{array}{l}\text { Disease } \\
\text { Staging }\end{array}$ & $\begin{array}{l}\text { Quality } \\
\text { Assurance }\end{array}$ & $\begin{array}{l}\text { Discharge } \\
\text { Abstract }\end{array}$ & Diagnoses & $>1800$ \\
\hline CSI & $\begin{array}{l}\text { Quality } \\
\text { Assurance }\end{array}$ & $\begin{array}{l}\text { Medical } \\
\text { Record }\end{array}$ & $\begin{array}{l}\text { Specific set of criteria } \\
\text { per diagnosis }\end{array}$ & $4 / 5$ \\
\hline APACHE II & $\begin{array}{l}\text { Quality } \\
\text { Assurance }\end{array}$ & $\begin{array}{l}\text { Medical } \\
\text { Record }\end{array}$ & $\begin{array}{l}12 \text { Clinical } \\
\text { Variables }\end{array}$ & 71 \\
\hline MEDISGRPS & $\begin{array}{l}\text { Quality } \\
\text { Assurance }\end{array}$ & $\begin{array}{l}\text { Medical } \\
\text { Record }\end{array}$ & $\begin{array}{l}500 \text { Key clinical } \\
\text { findings }\end{array}$ & $4 / 5$ \\
\hline
\end{tabular}

\section{Medical Meaningfulness/Content Validity.}

The requirement for patient classification systems to be medically meaningful is an important one. The medical meaningfulness of a classification can be defined as the degree in which a representative panel of physicians from a specific medical specialism may reach consensus on collapsing a number of similar or adjacent cases into a smaller number of aggregated patient groups, in such a way that these groups are still recognizable by other physicians not in the panel. The content validity refers to the degree in which the 
classification covers the range of all relevant medical case types.

However, the purpose of this collapsing procedure is not a medical one: the goals are set by economists who for instance want to explain costs of medical care, by epidemiologists who for instance want to explain survival or death of patients, by researchers who for instance want to compare the efficacy of alternative treatment types or who are interested in the effects of quality of care on the patient's state of body or mind. The resulting classification of patients will be different for each goal. It is very important that the classification of patients is validated by a panel of physicians, so the independence of measurement of the system in relation to its function is guaranteed.

It can be concluded that all patient classification systems have used clinical expertise to develop criteria to classify patients. However, it is not always clear which specific rules have been used by physicians in order to judge the clinical recognizability. It is likely that clinical recognizability will differ depending on the type of variables used to develop a patient classification system. More basic clinical indicators such as those used by CSI, APACHE II and MEDISGRPS may result in other ways of 'recognizing' patient types than the discharge diagnosis and surgical procedures that are used by DRG, PMC and Disease Staging.

Regarding the complexity of the matter, very few studies have been performed to judge the medical meaningfulness of a system. In a study conducted at the University of Michigan (Thomas e.a. 1986), a panel of physicians did evaluate the clinical meaningfulness by comparing the content validity of PMC, MEDISGRPS, APACHE and Disease Staging. The panel of physicians rated the clinical credibility of both APACHE II and Clinical Staging as high. However APACHE II was thought to be more valid as a severity measure for intensive care patients than for general hospital patients. The panel expressed reservations about the credibility of severity systems, such as Coded Disease Staging and PMC's, based on ICD-9cm codes. Thomas e.a. (1989) has evaluated also the construct validity of Apache II, Disease Staging (Clinical and Coded). MEDISGRPS and PMC's. Severity scores assigned 10 characteristics of individual patients by a panel of physicians were compared with the scores derived from each of the severity systems. MEDISGRPS compared most favorably with the ratings of the panel, suggesting that its score(s) will approximate the information used by experienced clinicians in evaluating patient's severity. Ranking behinc MEDISGRPS in descending order of construct validity were PMC, Clinical Staging and Apache II. The construct validity results for Coded Disease Staging were lower than for the other severity systems. In another study performed by Charbonneau e.a. (1988) it was found that physicians did not feel comfortatile with the concepts of DRG's, particularly with regard to patients with multiple illnesses. Disease Staging was thought to fit in relatively easily in the process of evaluating the patient's condition because of its familiarity with oncology. The idea of PMC's was thought to he more difficult to understand because it does not explicitly take the designated principal diagnosis into account in the categorization of patients. On the other hand, PMC's were found to be more consistent with standard medical nomenclature.

\section{Economic Meaningfulness/Predictive Validity.}

Another important requirement of patient classification systems is the degree in which it is able to explain medical care costs. A statistical technique which is often applied to measure the predictive validity is analysis of variance. The degree in which a system is able to explain variations in the dependent variable (such as length of stay or medical care costs) can be expressed by the $\mathrm{R}^{2}$ or Eta'; the higher these proportions the better the predictive validity. Results from the studies as described in this paragraph must be interpreted with care: $R^{2}$ values are not comparable across studies because they may be influenced by differences in populations. in operationalization and measurement and in time. Results from a study in which the different classification systems have been applied to the same data set of AMI patients will be described in table 8.7. (paragraph 8.3). 
Various studies (Mitchell e.a.,1984, Mitchell e.a. 1985 west e.a.,1985 , Coffey, 1985, Frank and Lave, 1985) showed that in general DRG's will. explain 16 to 18 percent of the variation in length of stay or costs in untrimmed data sets (outliers included). Between 26 to 33 percent of the variation in costs will generally be explained in trimmed data sets (outliers excluded). Within the DRG classification the surgical DRG's will provide most of the explanatory power. In trimmed data sets surgical DRG's will explain from 48 to 57 percent, while the nonsurgical DRG's tend to explain only from 7 to 16 percent of the variation in costs.

The predictive validity of the Computerized Severity of Illness has been tested by Horn (1987) in patients with 30 common disease conditions in 6 hospitals across the United States. Analysis of these data showed that five CSI levels explain 30 \% of the variance in length of stay. When CSI was determined within a group of patients undergoing a specific operating room procedure the predictive value increased 1067 percent. In another study (Horn, 1991) performed in five teaching hospitals using a sample of 2378 patients classified into 27 high-volume DRG's, it was found that the DRG's explained $27 \%$ of the variance in length of stay, while DRG's adjusted for admission-CSI-scores explained $38 \%$. DRG's adjusted for maximum-CSI-scores throughout the hospital stay explained $54 \%$ of the variance.

Comparing Disease Staging with PMC's and DRG's, Calore (1985) has found that neither PMC's nor Disease Staging explain costs better than DRG's. If one distinguishes nonsurgical patients from surgical ones, both PMC's and Disease Staging perform equally well as DRG's. Coffey (1985) found similar results and suggests that the failure of Disease Staging to improve on DRG's may be caused by the fact that both rely on the same medical information. According to Coffey co-morbidities and disease-specific severity indicators based on ICD-9-cm codes are insufficient to predict differences in hospital costs. Similar results were found by Calore and lezzoni (1987). After controlling for DRG's, both Discase Staging as well as PMC's add litte to the predictive value regarding costs for either pneumonia or prostatic disease. The authors conclude that application of either system as a DRG-modifier will not improve on the results of DRG's, but may identify patients within DRG's that clearly do not belong to that group. Also Charbonneau (1988) concluded that the Disease Staging system and the PMC system did not increase statistical homogeneity in length of stay within some specific DRG's (in this case acute myocardial infarction and respiratory problems).

Wagner (1984) has studied the relationship between the APACHE-score on admission and the total costs of treatment over the entire course of the ICU stay in 12 hospitals. The APSscore alone accounted for 38.6 percent of variance explained, and diagnostic variables and hospital identifiers together accounted for 24 percent of the variance explained regarding ICU costs. Data analysis for three diagnostic categories (drug overdose, peripheral vascular surgery and diabetes) has shown that a consistent and substantial increase in costs was significantly correlated to an increase in APS scores for each of the three diagnostic categories.

Brewster e.a. (1985) have studied the relation between the admission Medisgrps-score and costs for abdominal pain and chest pain; the mean total costs for both patient groups increase significantly for each successive severity group. The same results were found using the Medisgrps-score after 10 days; an increase in severity from admission to the second review had a significant effect, on costs. The researchers also tested the capability of Medisgrps to refine DRG's. The additional variance explained was 44 \% for DRG 140 'angina pectoris' and $40 \%$ for DRG 243 'medical back problems'. Brewster indicates that besides the 
admission severity, the increase in severity is an important factor causing cost differences within DRG's. Iezzoni e.a. (1988) found different results; the admission Medisgrps score alone explained only $3 \%$ of costs using trimmed data. Addition of Medisgrps score to DRG's modestly improved the explanatory power for both costs and length of stay. Using untrimmed data the explanatory power was higher. Iezonni suggests that the relatively better performance among untrimmed cases might result from the ability of admission Medisgrps score to predict the costs of severely ill outliers. Within individual DRG's the Medisgrpsclassification on admission explained the variance in costs ranging from 0 to $21 \%$. Iezonni did not use a second review score in this study. This may account for the differences between her results and the results found by Brewster.

Thomas e.a. (1986) have compared the predictive validity of four severity measurement methods: Apache II, Disease Staging (Clinical and Coded), Medisgrps and PMC's. The study focuses on the explanation of cost variations within a limited number of DRG's. To obtain a broad representation of cases, adjacent DRG's (ADRG's: diagnosis or surgical categories not subdivided into DRG's using age or complication and co-morbidity criteria) rather than single DRG's were chosen. The authors conclude that in almost all ADRG's at. least one of the severity measures (and usually several of the measures) provides higher $\mathrm{R}^{2}$ values than those obtained with DRG's. The increase in variance explained, however, will vary per severity measure and per ADRG. In a recent study performed by the same author (Thomas, 1991) APACHE II, Medisgrps, CSI, Disease Staging and PMC's were compared in terms of how well they are able to explain variations in observed costs among patients. All severity systems were found to improve on DRG's for some types of cases, but to offer litile or no improvement in others. Indicators of maximum severity, especially maximum CSI, did explain greater proportions of cost variation than measures of admission severity and measures based on discharge abstracts.

\section{Reliability and practicality.}

In two articles by Thomas e.a. $(1986,1989)$ the inter-rater reliability of four severity measures (Apache II, Disease Staging (Clinical and Coded), Medisgrps and PMC's) as well as the potential for measurement error have been examined. The highest observed reliability was achieved by Apache II and Medisgrps. Clinical Disease Staging was found to be more reliable than Coded Disease Staging and PMC. Remarkable is that the latter two systems which were found to be relatively more unreliable, are the ones which use medical discharge data exclusively.

Resulis of the comparison for the potential for measurement error showed that Apache II and Clinical Staging were viewed as relatively less erroneous. Measurement erior in patients* discharge records by listing additional diagnoses and/or procedures, in either Coded Disease Staging or PMC's is likely to be no larger than that in DRG's.

The same authors have also compared the more practical issue of the costs of implementation and operation of patient classification systems. Their conclusions are that neither Coded Disease Staging nor PMC's have any additional time requirement over that required for the discharge abstract. Apache $1 \mathrm{I}$ requires about 11 minutes and Clinical Staging required about 12 . The admission review with Medisgrps required on average 16 minutes per record. Computer processing costs of PMC's and Coded Disease Staging are negligible and no additional data entry is required. Medisgrps. Apache II and Clinical Staging do require computer processing time for data entry but costs are supposed to be relatively low. 


\section{Conclusions.}

Examining and evaluating the patient classification systems described in literature one may conclude that each system has advantages and disadvantages. Generally speaking a major distinction can be made between (1) illness-specific classification systems that classify patients by discharge data; their original purpose is prediction of length of stay and/or costs of medical care and (2) severity of illness classifications based on specific clinical indicators, originally developed to predict mortality; these systems are used for quality assurance programs.

Patient classification systems relatively easy to apply because of their use of computerized discharge variables (DRG's, PMC's and Coded Disease Staging) will predict variations in hospital cost to some extent. The predictive power turns out to be much higher for surgical patients than for diagnostic (non-surgical) patients. Severity measures using clinical indicators (such as CSI, Medisgrps and Apache II) may sometimes improve cost prediction, although these systems were originally not developed for this purpose. Measurement of changes in a patient's severity of illness during hospital stay may contribute even more to the explanation of costs than one unique, period-bound score intended to represent a patient's severity of illness during his or her whole hospital stay. Assuming that the severity of illness is useful in explaining variations in cost amongst patients it is reasonable to expect that its value will be greatest when examining patients with similar medical conditions. It may be assumed that the addition of a severity measure hased on the results of clinical findings will improve the explanatory power of patient classification systems, which are based on medical discharge data.

The various severity criteria used in severity systems are mainly derived from sessions with panels of physicians in order to predict mortality. Studies in which sets of severity indicators themselves are also empirically tested to explain costs of medical care are rare. It is likely that within a similar diagnosis group, mortality or quality of eare will be explained by other severity indicators than those explaining costs of medical care. As can be seen from the examples of classifying patients with acute myocardial infaretion, these systems each use different severity criteria to categorize patients into a group. It may be concluded that severity of illness is not an absolute or universal notion and much is still unclear about how to define and operationalize this rather complex concept.

The measurement of severity of illness in relation to cosis of medical care must be seen as an ongoing process which will cost years of sudy and analysis by many researchers. In the forthcoming chapters an attempt will be made to measure this concept for patients with Acute Myocardial Infarction (AMI). The severity of illness will be defined and operationalized independently of already existing systems. We feel that systems that are not originally developed for cost explanation are less suitable in the context of this study. So we have made a fresh stari at defining and measuring severity of illness. In the fortheoming chapters relations between the severity of illness and cost of medical care will be investigated and finally a Severity of IIIness classification specifically developed to explain cost of medical care in AMI patients will be proposed. 



\section{Explanation of medical care costs.}

\subsection{Study objective and definition of problem.}

A precondition for setting up models for medical management is having insight into the factors that will cause cost differences in medical care. These factors will be manifold.

It is very plausible that illness characteristics of a patient are the most important factors causing costs differences between clinical patients. Next to this, factors not related to the patient's illness may produce cosi differences. Characteristics of internal hospital processes or the way in which hospital and medical departments are organized may also be responsible for cost differences. In addition, there may be external factors responsible for cost differences. The medical care given in hospital is only part of the overall care given to a patient. Other types of care are provided by health care facilities like home care, general practitioners, ambulatory care and long term facilities. The extent to which health care was provided by other health care facilities outside the hospital will influence the medical care that patients receive in hospital (Van de Ven c.a., 1980).

The main interest of this study is to explain differences in costs of medical care caused by illness characteristics of clinical patients. Classification of patients in terms of their illness may be seen as an important step toward reaching this goal. Litcrature (see chapter 2) reveals that patient classification systems using discharge diagnosis and surgical procedures do not quite succeed in achicving a high level of homogeneily in medical care costs within defined patient categories. Economic homogeneity is generally considered to be an important goal for evaluating the accomplishments of a classification system. Literature also indicates that once a patient group has been delineated, more sophisticated instruments which aim to measure a patient's severity of illness may increase the homogeneity of costs.

It is plausible that the typical medical deficiencies of a patient play a central role in the explanation of cost differences within patient groups defined by diagnosis. Thus, a patient classification system should place the characteristics of a patient regarding his/her typical medical deficiencies in a more prominent position. However, a person's illness condition may change during hospitalization as a result of 'natural' autonomous processes or in response to medical treatment. A prior illness condition will, for example be followed by certain medical care (and medical care costs). This medical care will generally ameliorate the condition of a patient in a next phase. The cyclic character of the causal relations between severity of illness and (costs of) medical care is presented in diagram 3.1.

The dynamics of change in the severity of illness may follow certain patterns in specific groups of patients. For instance, patients may be severely ill on admission but may not develop complications during hospitalization. Other patients may initially have a moderately severe condition, but may develop severe complications. Consequently, a different process of diagnostics and treatment will be part of the patient's hospital stay. The illness condition of a patient. and the changes therein during hospitalization may be seen as the most important factor for differences in costs. Therefore, a longitudinal, dynamic model describing the patient's stay will be appropriate in order to study the severity of illness in relation to medical care costs. Although some of the severity systems described earlier may have found ways to incorporate changes in severity of illness during hospital stay, they generally fail to do this in a reliable and valid fashion. In these systems patients are assigned to severity levels based on a set of global physiological indicators. Severity of illness is usually measured by a unidimensional index using the patient's most deviating scores on certain severity indicators. The mutual relationships between the severity indicators are: not medically validated; the individual 
effects of specific severity indicators on specific costs are also not empirically analyzed or tested.

Diagram 3.1. Dynamic causal model of relations between severity of illness and costs of medical care during hospital stay for pre-determined diagnosis groups.

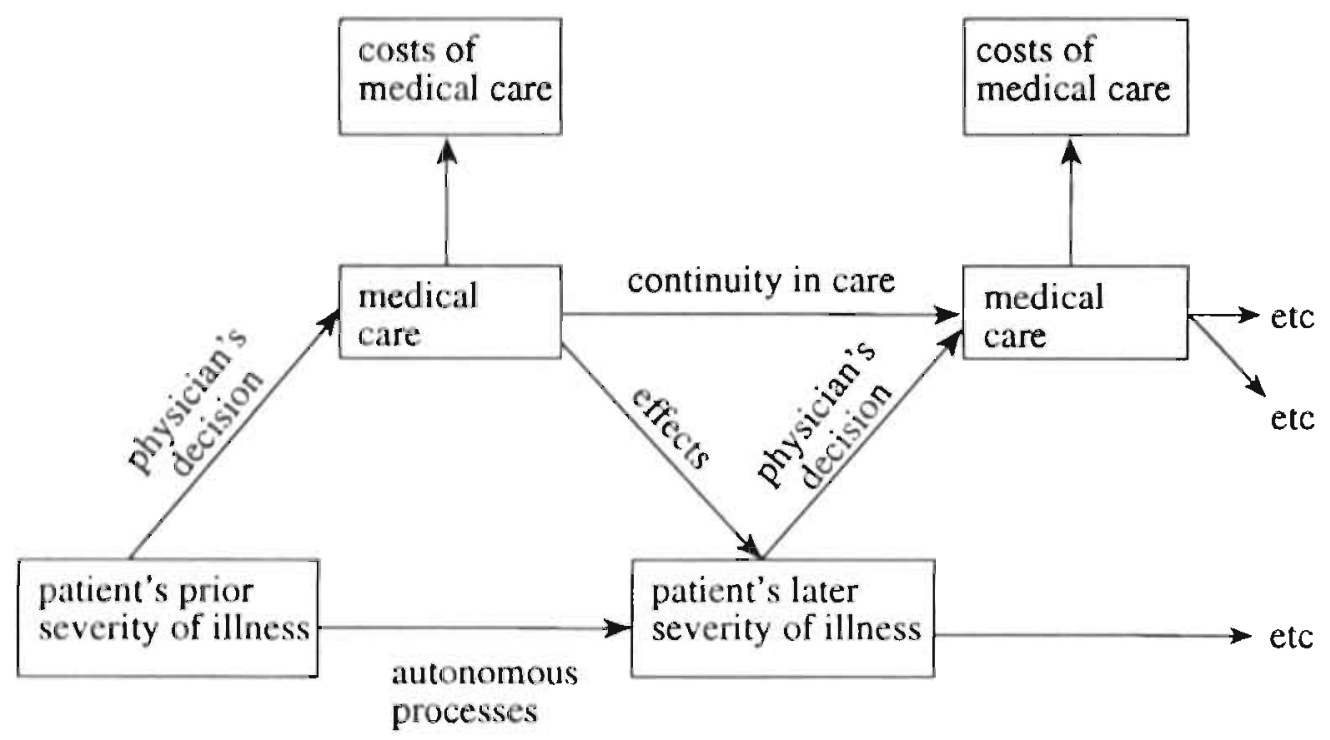

It is assumed that measurement models which, first of all, specify the interrelations between medically defined indicators of severity of illness offer a better approach to explaining cost diflerences. Subsequently, these measurement models should be condensed and trimmed by ceonomists to be used in the explanation of costs during hospital stay. This latter process should be done without impairing the medical meaning of the indicators and should result in a more refined and flexible classification of patients.

The objective of this study is to investigate the factors which may explain the differences in costs between clinical patients with similar discharge diagnoses. Applying a longitudinal multivariate model of explanation is assumed to be effective in reducing the heterogeneity of costs within patients with similar discharge diagnoses. The DRG-system will be used to calegorize patients into discharge diagnosis groups. For a selected number of DRG's the effects of severity of illness on differences in costs of medical care during hospitalization will be investigated. In a dynamic model, relations are hypothesized between, on the one hand the different indicators describing the severity of illness of a patient, and on the other hand costs of medical care in hospital. By using a flexible and dynamic definition of Severity of lllness. a new patient classification system will result, aimed at explaining differences in costs in a more detailed and clinically valid way. 


\subsection{Factors which explain differences in medical care costs.}

In general, medical care costs for clinical patients can be divided into three major cost categories: treatment costs, diagnostic costs and nursing costs. The classification using (1) severity of illness may have different effects on each of these costs categories. But next to this, (2) background characteristics (3) contra-indications for treatment, (4) research protocols and (5) admission and discharge characteristics of patients are also assumed to play an important role in explaining differences in cosis.

\section{Severity of Illness.}

At present, diagnosis groups are specified after discharge according to the International Classification of Diseases, 9th revision, clinical modification, (ICD-9-cm) coding scheme. These codies are used to categorize patients according to the DRG classification. However, the retrospectively given codes obviously fail to measure a patient's total burden of illness during hospital stay in an adequate and valid way. Diagnosis codes do not take into account the periodical changes in a patient's health state during hospital stay. The classification process places the patient into a category. which crudely summarizes the severity of illness. The patient's complications or co-morbidities (secondary diagnoses) are not sufficient to describe the severity of illness. A better approach to defining a patient's total burden of illness during hospital stay would be provided by a severity of illness classification defined in terms of a time-dynamic set of scores that deviate from 'normal' on a complex of clinically interrelated medical deficiencies. The deviations from 'normality' may be weighted by a panel of physicians. A high score on a weighted index will correspond with a more severe illness condition. It stands to reason that in comparison with relatively mild cases, more severe cases are medically complex (have higher scores for many deficiencies) and have a relatively bad prognosis. Consequently, patients with more severe conditions may need more intensive medical care, and may have a longer hospital stay. In both instances, this will produce higher costs of care.

Most costs in medical care are generated by the diagnostic or treatment policy of the physicians. It may be assumed that the physicians' decisions in these matters are cventually caused by and based on severity characteristics of patients. In other words: a severity of illness classification will explain the differences in costs of medical care between patients more successfully than the DRG-classification.

\section{Background characteristics of patients.}

Clinical patients will differ in their pre-hospital medical history or in individual characteristics. From a clinical point of view, a patient's medical history before hospital admission (ineluding former hospital stay) may require the physician to pay special medical attention to that patient. For example: in comparison to other patients, patients with chronic deficiencies like diabetes mellitus will need special care. when they are admitted to hospital. In addition to this, individual patient characteristics may influence a physician's decision policy in providing care. Differences may exist between costs for young patients and old patients with the same medica! problern. Ultimately, a thorough understanding of the severity of illness during hospital stay can only be acquired if cat takes into account the complex interplay between decision making policy (protocol) and these patient characteristics. Thus, the conclusions from above may be stated as follows: specific background characteristics of patients may also be responsible for differences in medical care costs, given their severity of illness. 


\section{Contra-indications for medical treatment.}

Specific patient characteristics, such as high-risk indications, may restrict a physician's decision to provide certain care. These restrictions from specific medical care will be applied if a patient has one or more so-called contra-indications. Contra-indications may be connected to specific individual patient characteristics or events in the individual medical history. Consequently, it may be concluded that contra-indications for diagnostic or therapeutic services will constrain or preclude specific care to be given to a patient. In such instances, cost of medical care may be relatively low, in spite of the patient's severity of illness.

\section{Research protocols.}

In many university hospitals clinical trials are held in combination with (or next 10) patient care. If patients participate in such research projects, a specific research protocol has to be followed. Consequently, for these patients the costs of diagnostic tests and medical treatment may differ, in type and amount, from treatment or tests given to patients not in the research project. In other words: costs of medical care may vary between patients with similar diagnosis depending on the patients' involvement in and compliance to a specific research protocol. Enrollment of patients in a research project may partly be responsible for differences in costs of medical care, regardless of the patient's severity of illness.

\section{Admission and discharge characteristics of patients.}

The way patients enter and leave the hospital will difler from one patient to another. If patients are transferred from other hospitals, they will generally have lower costs than patients who are admitted regularly or who come in by ambulance. Patients will show differences in discharge too: if patients are transferred to other departments of the same hospital their costs at the admission department will generally be lower than patients who have a 'regular' discharge. Also individual decision making of physicians to discharge a patient may vary from patient to patient.

When patients die during hospital stay, they will generally have lower costs than nondeceased patients. The purpose of this study is, among others, to develop a severity of illness classification which explains costs of medical care. Such a classification is not intended or fit to explaiñ a non-regular admission or discharge (like mortality). Thus, characteristics of moment of admission to and discharge from the hospital may be seen as additional factors in costs explanation. 


\subsection{Models for explaining differences in medical care costs.}

It may be clear from the above that many factors relevant for explanation must be included in the analysis of the problem; usually this is done in multivariate models. In the development of such cost-explanation models several methodological issues have 10 be considered. A patient's condition, measured in terms of his/her severity of illness, will be subject to changes during hospital stay as a result of autonomous processes or medical treatment. The objective of this study is to develop a model which will explain medical care costs in terms of the possible changes in severity of illness. The steps in a physician's decision-making process as well as the patient's changing condition will have to be translated into the model's concepts. To realize this objective the model should be representative for the illness condition of the patients in all relevant time periods during hospital stay. With these considerations in mind, two different models aimed at explaining differences in cosis of medical care are proposed. Firstly a period-specific model will be presented which can be seen as a specification of the model in Diagram 3.1, and secondly a dynamic model for the whole stay will be given. Both models will be used to analyze differences in medical care costs for patients with acute myocardial infarction (see chapter 7).

(1) A model explaining differences in period-specilic costs of medical care using a period-specific Severity of Illness definition.

The first model (diagram 3.2) explains differences in period-specific costs of medical care by a patient's period-specific severity of illness. In this model the hospital stay has been divided into several time periods (0-2). The number of time periods will strongly depend on the type of illness within a patient population (for instance chronic versus acute illness). For each time period costs of medical care are distinguished into diagnostic costs and treatment costs. It is assumed that the costs for the first diagnostic investigations will depend only on the direct cause of admission. The provision (and costs) of medical treatment on admission may be directly dependent on the patient's severity of illness on admission and on specific: indications or contra-indications. Costs for further diagnostic investigations will be influenced by the severity of illness at admission and the given medical teatment. Contra. indications are assumed to affect costs of treatment in every period, regardless of the patient's severity of illness. Costs for additional medical treatment in a next phase of hospital stay may be explained on the same basis. It is also expected that former medical treatment and the period-specific severity condition of a patient will form the basis upon which a physician makes decisions. If patients are admitted to a research project in which a specific research protocol has to be followed, this will constrain the physician's freedom of choice in diagnostics or treatment. 
Diagram 3.2. Period-specific model explaining differences in costs of medical care during hospital stay for pre-determined diagnosis group(s).

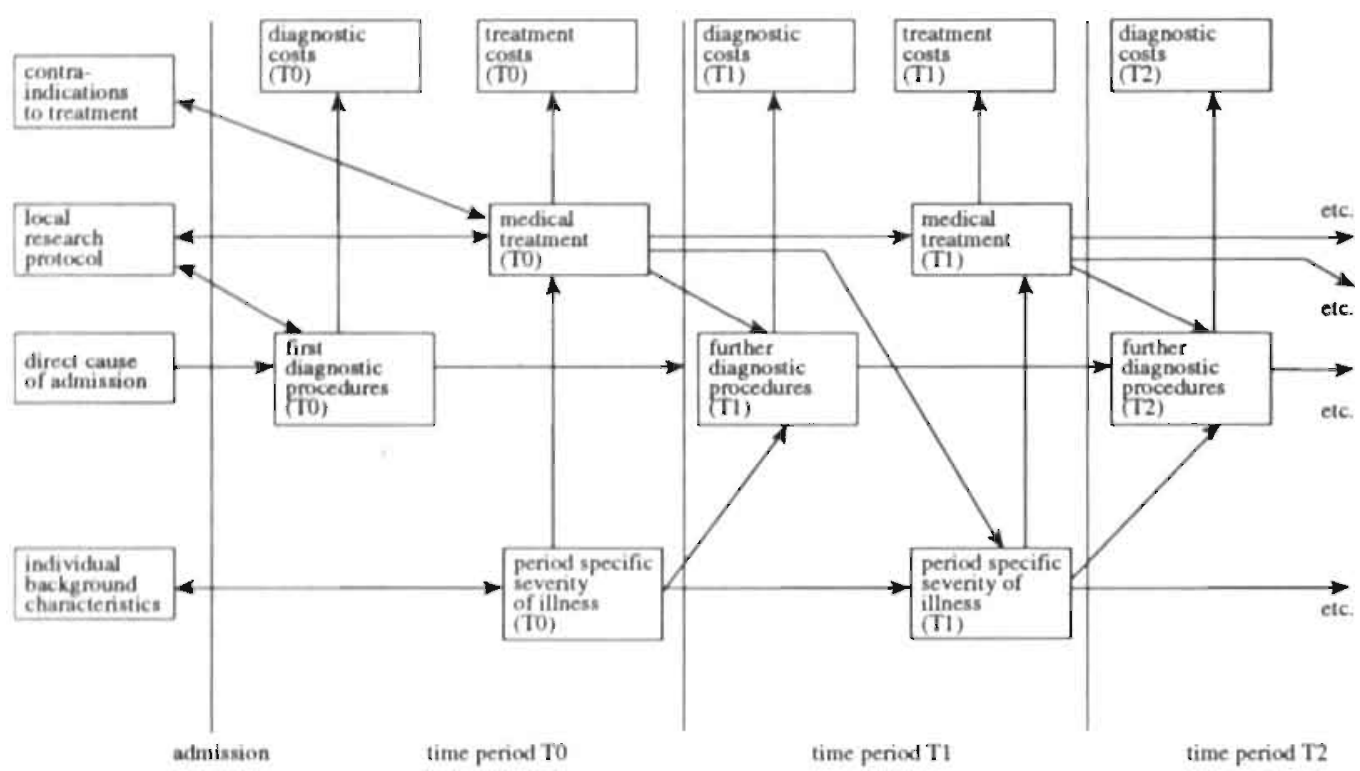

This model concentrates on period-specific analyses of costs; costs made within a cerain lime-period during a hospital stay are explained on the basis of a period-specific severity of illness. This approach is relevant if medical care costs can be ascribed to a specific time period within the hospital stay. However, if costs of medical care pertain to the entire hospital stay, this model will be too laborious. Consequently, an aggregated model for the tot:l hospital stay is also proposed.

\section{(2) A model explaining differences in costs of medical care during total hospital stay using a dynamic Severity of Illness definition.}

The second model (diagram 3.3) explains differences in total costs of medical care by a dynamic definition of severity of ilmess. The illness condition of a patient and the changes therein during hospital stay may be seen as the most important causal factor for differences in costs of medical care accumulated over all periods of hospital stay. Patients, with a similar diagnosis may show different courses of illness during their hospital stay: If the severity of the illness is measured repeatedly over several time periods, a severity pattern may be estublished for each patient. Patients with similar severity patterns may be grouped into the same severity category. This severily category then will summarize the degree of severity plus the (possible) changes herein during hospital stay. In this case we will speak of a dynamic Severity of Illness grouping. These severity categories may explain differences in the accumulated total costs of diagnostic procedures. medical treatment and nursing care during hospital stay. Possible influences of background characteristics, local research protocols, contra-indications and type of admission and discharge are taken into account in the same way as in the first model. 
Diagram 3.3. Model explaining differences in summarized cosis of medical care during total hospital stay for pre-determined diagnosis group(s).

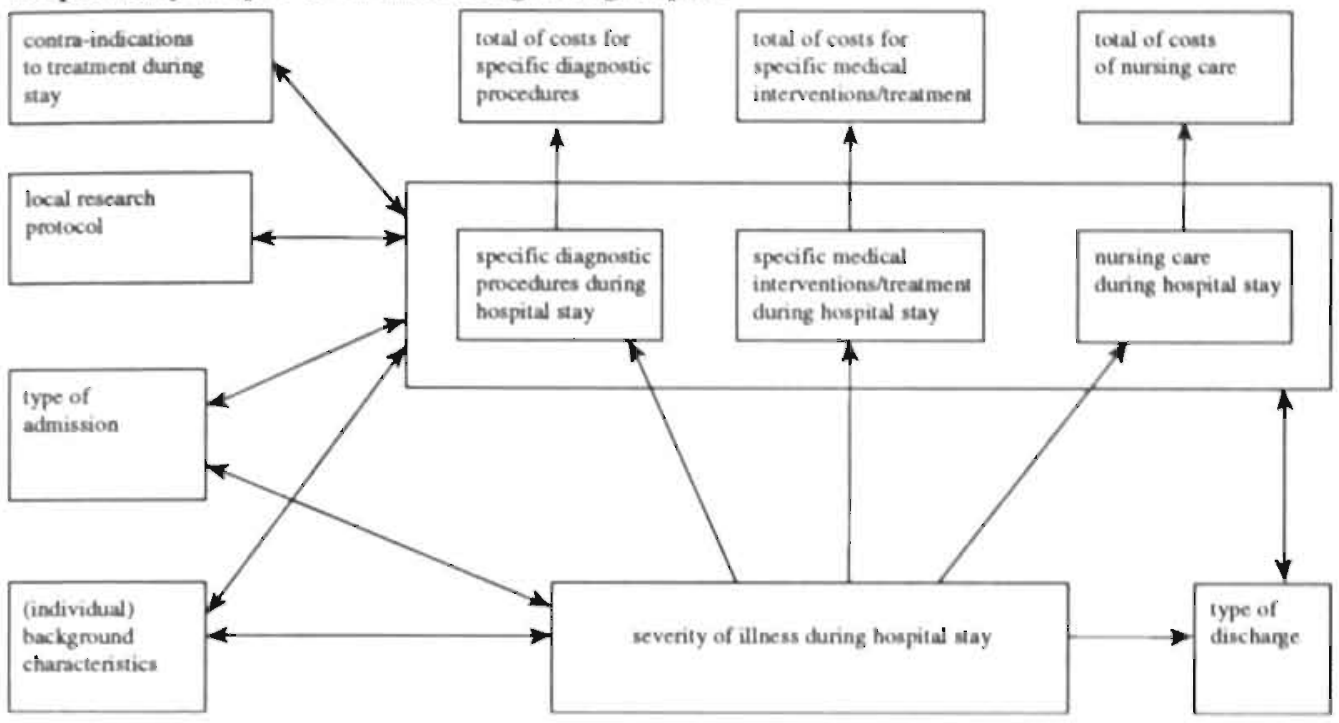

\subsection{Adaptation of the models to patients with Acute Myocardial Infarction (AMI).}

\section{Hospital Time periods.}

To assess the effects of severity of illness in relation to hospital costs it is proposed to divide the patients' hospital stay in specific hospital time periods. The way the time periods will be distinguished may be very crucial for period-specific costs analysis and the establishment of a dynamic severity of illness classification.

This study concentrates on patients with acute myocardial infarction who have not undergone cardiac surgery during hospital stay. For a full description of the patient population see chapter 4. Establishing the length of the time periods is strongly dependent on the type of patients under investigation. The patient population in question is characterized by an acute and sometimes life-threatening situation. From sessions with cardiologists it was concluded that the hospitalization period of these patients can be distinguished into four different phases:

\section{(0): The acute phase of myocardial infarction.}

When a patient with suspected myocardial infarction is admitted to hospital, the first hours of symptom appearance: are very crucial. Diagnostic tests are performed to ascertain the patient's type of diagnosis and severity of illness. Next, medical intervention will be performed. One of the most important interventions is the administration of thrombolytic drugs which may have a dramatic impact on the course of the illness. This intervention is only effective, if applied within the first six hours of symptom appearance. There may be a delay between the acute symptom appearance and the moment of admission in hospital, but this is very difficult, if not impossible, to measure.. For reasons of practicality the first six 
hours after admission to the hospital will be defined as the acute phase of the infarction. If patients are already hospitalized the first six hours after typical ischemic pain will be relevant.

\section{(1): Evaluation phase.}

The evaluation phase is defined as the period starting from six hours and ending three days after the moment of hospital admission (or ischemic: pain during hospital stay). During this period the severity of myocardial infarction is expected to become clinically manifest. Patients will normally stay monitored intensively in a coronary care unit during this period. Further specific diagnostics may be applied to assess the magnitude and impact of the infarction.

\section{(2): First follow-up phase.}

The first follow-up phase starts after three days and ends ten days after the patient's moment of hospital admission (or ischemic pain). During this period patients are generally placed in medium or low care wards. Specific tests may be applied to exclude any risks of postinfarction complications. In case of a minor infarction, and if there appear to be no further complications, patients are normally discharged from hospital within this period.

\section{(3): Second follow-up phase.}

The second follow-up phase theoretically starts ten days after the moment of hospital admission (or ischemic pain) and ends when the patient is discharged from hospital or is transferred to another medical department in the same hospital (see 4.2). It is assumed that these patients will develop complications during their hospital stay.

Thus, hospital stay for Acute Myocardial Infarction-patients is divided into four hospital lime periods: the acute phase of infarction (henceforth TO), the evaluation phase (henceforth T1) the first follow-up phase (henceforth T2), and the second follow-up phase (henceforth T3). These four hospital lime periods will be incorporated into the general models cxplaining hospital costs (figure 3.2 and 3.3 ).

\section{Medical care costs.}

The major objective of this study is to investigate the relation between severity of illness indicators in patients with myocardial infarction and costs of medical care. Patients with myocardial infarction will gencrally reccive various types of medical care. Diagnostic procedures mainly consist of laboratory tests, electrocardiographic tests (ECG's), heart catheterization, echocardiography and excrcise tests. Major medical interventions such as administration of drugs (amongst others thrombolytic therapy) and performance of Percutaneous Transluminal Coronatry Angioplasty (PTCA or dottering) may take place. The length of the patient's stay in hospital may be seen as an indicator for nursing care costs. Nursing care costs may be extra high, if patients stay at the Coronary Care Unit (CCU). The measurement of medical care costs for the patient population at hand is described extensively in chapter 6 .

The relation between the individual patient's background characteristics, contra-indications, enrollment in medical trials, type of admission and discharge and the patient's severity of illness for AMI patients will be empirically analyzed for different types of costs. In chapter 5 and 6 the models discussed in the previous paragraph (figures 3.2 and 3.3) will be further specified and operationalized for patients with acute myocardial infarction. In chapter 7 , the relations as described in this chapter will he investigated and analyzed. 


\section{Patient population and data collection.}

\subsection{Patient population.}

The survey population consists of patients admitted to and discharged from the Department of Cardiology of the University Hospital Maastricht between the first of January 1987, and the 30-th of April 1988. Patients were retrospectively selected from the Diagnosis Related. Groups System, categorized in the DRG's 'Acute Myocardial Infarction' (DRG 121, 122 and 123). These three DRG's are defined as hospital admissions of patients with a primary' or secondary diagnosis of acute myocardial infarction, in which no cardiac surgery was performed during hospital stay'. Patients with cardiovascular complications (like heart failure, rhythm and conduction disorders) are categorized as DRG 121 'Complicated AMI': patients having no other cardiovascular problems are categorized in DRG 122 'Noncomplicated AMI'. Patients, who died during hospital stay are grouped under DRG 123 'AMI deceased'.

In total 557 hospital admissions were categorized into these three DRG's. For patients with multiple hospital stays during the investigated period only the last hospital stay was taken into account $(n=10,1.8 \%)$. This was done because the unit of observation and analysis of this study is the patient and not the admission (period). The aim is to investigate differences between patients rather than differences between hospital admissions. During the clinical data collection (see $\$ 4.3$ ) it appeared that a total of 49 patients $(8.8 \%$ ) had incomplete or missing medical records; they had to be excluded from further study. There were 6 patients (1.1\%) who were admitted and discharged by departments other than cardiology; they were also excluded. For the remaining patient population close observation of the medical record revealed that 28 patients $(5.0 \%$ ) were misclassified as DRG's 121,122 or 123 , because of coding errors or inappropriate diagnosis. The most frequently found errors were:

patients with unstahle angina pectoris were coded as having acutc myocardial infarction $(n=17)$;

patients with an old myocardial infarction were coded as having acute myocardial infarction $(n=7)$;

patients with suspected acute myocardial infarction as recorded in medical record abstracts, but whose diagnosis was not confirmed in the patient's medical record $(n=4)$.

In total 464 patients $(83.3 \%$ ) of the original patient population as defined by the DRG's were investigated. About $30 \%$ of them were women with a mean average age of 68.1 ycars and $70 \%$ were men with a mein age of 60.5 ycars. The patients had a mean length of stay of 11.5 days with large variations betwecn patients (std.dev. 9.6). A large group of 310 patients $(66.8 \%)$ was classified as DRG 122 'Non-complicated AMl' with a mean length of stay of 10.1 days ( std.dev $=4.7$ ). The second largest group of 102 patients $(22.0 \%)$ was classified as DRG 121 'Complicated AMI' with a mean length of stay of 18.1 days (std dev $=16.1$ ). The smallest group of 52 patients (11.2\%) died during hospital stay and were grouped under DRG 123 'AMI deceased' with a mean length of stay of 6.4 days (std.dev=7.7).

\subsection{Hospitalization characteristics of the patient population.}

The way patients enter and leave the cardiology department may differ from one patient to the next. Hospitalization flow characteristics for the patient population at hand are presented in figure 4.1. The hospital stay at the cardiology department is divided into four time periods

1 Patients with cardiac surgery are placed in other DRG's. Costs of these patients must be analyzed separately from patients who had not have surgery. 
(T0 to T3) as defined in our model of explanation. The first period (T0) concerns the first six hours of stay during acute myocardial infarction. Almost all patients in the population $(n=447)$ were admitted to the hospital during this period. A sub-group of them, namely 14 patients, was already staying in hospital. During the first six hours of the infarction 10 of the 447 patients died. In the second time period (T1) 6 'new' patients entered the cardiology department. The latter group of patients came from other hospitals: they were admitted after the acute phase of the infarction. In total 28 patients 'left' the department in this period: 19 patients died, 3 patients were transferred to other departments, and 6 patients were discharged from hospital. Between 3 days and 10 days after the infarction (period T2) another 8 patients were admitted to the cardiology department. In this same period a large group of patients $(n=238)$ was regularly discharged from hospital, 14 patients died and 4 patients were transferred to other departments. 167 patients stayed longer than 10 days (period T3), while 3 patients were admitted from other hospitals at a later stage in the infarction. The majority of these patients $(n=162)$ were discharged from hospital; in this period 7 patients died and only 1 patient was transferred to another department.

Flgure 4.1. Hospltalization flow characteristics of patient population.

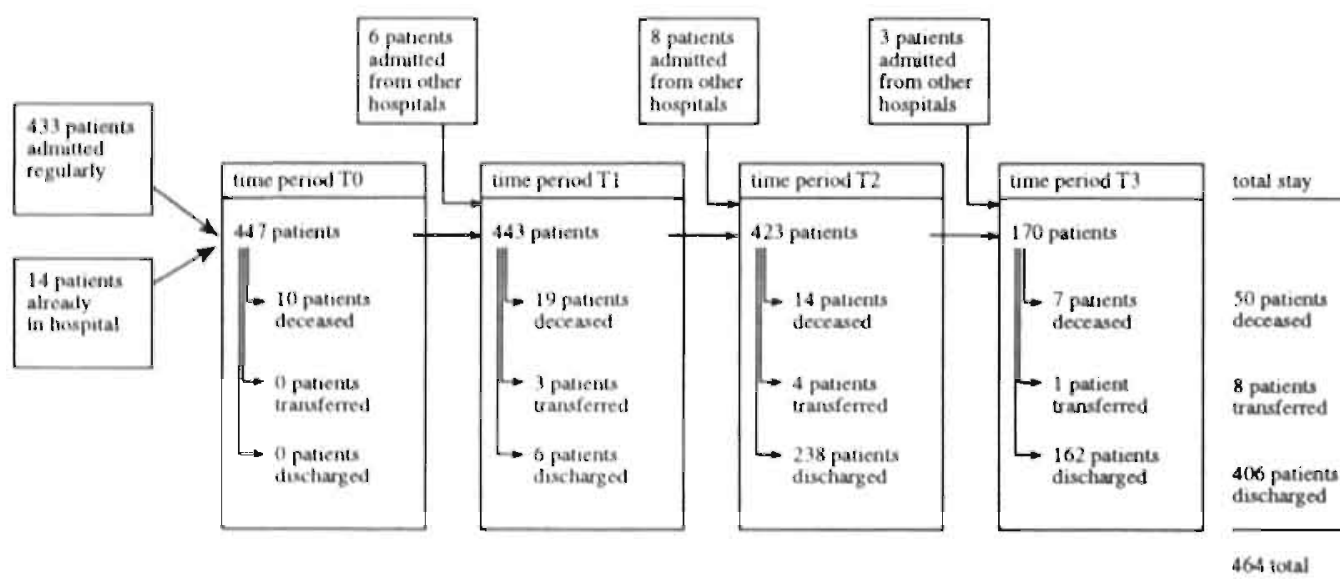

\section{* The admission.}

AMI patients are usually admitted to the hospital in the regular way: they enter the hospital directly arriving from non-hospital environment (home, work, etc.). Some patients may get the infarction while already in hospital. Patients may also enter the hospital via other hospitals during various stages of their illness. For the patient population, the type of admission was specificd as: 'from home'. 'already in hospital' and 'admitted via another hospital". If patients already were in hospital when they began to show symptoms of an acute infaretion this was indicated with a special variable (ADINTO; internal admission in TO). Furthermore, if patients were admitted via another hospital, the stage in the infarction upon entering the hospital was important. A distinction was made between patients entering the hospital during the evaluation phase of the infarction (T1), the first follow-up phase: (T2) and the second follow up phase (T3). For the patients entering the hospital in this irregular way, separate variables were constructed (ADEXT1: admission external in T1, ADEXT2: admission external in T2 and ADEXT3: admission external in T3) 


\section{* The discharge.}

The majority of the patients were discharged from the cardiology department in the regular way (during T2). Patients with a non-regular discharge were characterized respectively by variables HOMEl. (discharged to home within three days) and HOME3 (discharged to home after 10 days). Due to the severity of AMI it was assumed that no patient would be discharged during the acute phase (TO). Patients might also have been transferred to another medical department in the same hospital. In this case, the moment of transfer was characterized by the following variables: TRANS1: transferred during T1, TRANS2: transferred during T2 and TRANS3: transferred during T3. For deceased patients also separate variables were constructed namely: DEATHO; deceased during T0, DEATH1: deceased during T1, DEATH2: deceased during T2 and DEATH3; deceased during T3.

\subsection{Data collection.}

Two basic sources were used to obtain the necessary data in this study: computerized data from the hospital administration deparments and non-computerized data from medical records of patients.

\section{* Computerized data.}

The computerized data collection comprises partly medical discharge variahles and partly billing variables.

Medical discharge variables have been collected in Dutch hospitals during the past decades and are used for general and specific hospital purposes. In the majority of Dutch hospitals these data are registered and coded according to national guidelines from the 'Landelijke Medische Registratie' (LMR registration). For every hospital admission there is a medical abstract record which contains information on general and specific patient characteristics (e.g.: age and sex), clinical characteristics (e.g.: diagnosis and surgical procedures), medical specialty (e.g.: admission and discharge specialty) and hospital stay (e.g.: length of stay). These data are collected by trained medical record personnel from the physician's discharge letter and from information in the patient's medical record. Some of this information is used for categorizing patients into DRG's according to the 1985 version of the Yale DRGgrouper. Finally, the patient abstract record is extended with specific DRG variables like the MDC code (Major Diagnostic Category) and the DRG-code (Diagnosis Related Group).

The computerized billing variables measure the type and amount of resources consumed during an episode of patient care. The hospital billing administration office normally sends. out bills or submits claims for payment to insurance companies. All hospital activities that can be charged are collected, coded and entered into the billing system. For this survey, the billing records are converted into individual patient-reseurce records to obtain information on the resource use per patient. Subsequently the number and type of services are calculated for each patient.

\section{* Medical Record Data.}

The repository of clinical information in a hospital is the patient's medical record. While a patient is in hospital, physicians and nurses enter clinical and other information inte the patient's record. Transfers among hospital departments, laboratory test results, operating room notes, medication orders, standardized anamnesis forms and ali other relevant clinical information of a patient are gathered in the medical record. The patient's medical record is a handwritten and/or typed documentation file in which several papers, documents and reports regarding to the patient's hospital stay are compiled. The content may differ from one medical department to another. After discharge the records are kept in the central archive of 
the medical administration department.

In this study medical records were the main source of clinical information. The data required for this study were registered using a specially designed registration form containing more than 600 items (see appendix 1). Each registration form consisted of a title page on which patient number, name, admission and discharge date were noted. Next, items concerning the medical background, physical examination, results of diagnostic tests, medication and therapeutic interventions were collected and coded according to a previously constructed code book. In this registration form the patient's cardiovascular and cardiac history and risk factors were collected. If patients were participating in a research program during the hospital stay this was also recorded. Next, items concerning the current hospital stay, repeated for every defined hospital period (T0-T3), were collected. These items are collected from (1) the medical history, like the duration of ischemic pain and Angina Pectoris Class and from (2) the physical examination, like rales, edema and blood pressure. Furthermore, (3) ECG data, like the location of Q-waves and ST-changes, but also the type and frequency of several rhythm and conduction disorders were included. Next, (4) coronary stenosis measured by coronary angiography. (5) different enzyme rates and the duration of an enzyme peak were collected. Also, (6) wall motion abnormalities and heart failure measured by echocardiography, (7) administrated drugs, (8) treatment such as thrombolytic therapy and PTCA and (9) specific relevant complications, such as CVA, mitralis insufficiency etc. were included. Finally, (10) results from exercise tests, according to the Bruce protocol and thallium test were recorded. As general guideline, the most deviating score in each observed time period has been taken as the definite score. In case of missing, unknown or illegible information special codes were ped

cuitutions of eitors in wata gathering were made atter the registration form was completed. Still missing data were traced as much as possible and incorrect codes were rectified. Subsequently, the coded data were entered into a database. Due to the medical complexity of interpretation and registration, this part of data collection was performed by specially instructed medical personnel. The time spent transcribing scores from an average medical record took approximately an hour and a half per patient.

\subsection{Quality of data.}

- Medical Discharge Data.

A study on the quality of medical abstract records in The Netherlands has been performed by the 'Stichting Informaticvoorziening voor de Gezondheidszorg' (Gemert, 1986). In administrative variables, like age, insurance, acuteness indication at admission, the pereentages of misclassifications were lying between $0.5-1.5 \%$. Unfortunately the percentage for 'misclassificd diagnosis" was much higher: sequencing errors occurred in 3.4 $\%$ of the cases, a "vague' principal diagnosis occurred in $4-6 \%$ of the cases and a similar pereentage of the cases showed an inconsistent relation between diagnosis and surgical procedures. With respect to the surgical procedures only $1-2.5 \%$ appeared to be incorrect. In our research population we found comparable results. These results corroborate the results from other studies outside the Netherlands (Connell 1984, Corn 1980, Doremus 1983. Johnson 1984. Lloyd 1985. Young 1986). 


\section{* Billing Daa.}

Expectations are that billing data are more reliable than medical discharge data, since billing data are carffully processed by both the hospital and the insurance companies. There appears to be no adequate literature on the quality of billing data in Dutch hospitals. In the hospital under observation the percentage of misclassified billing data lies approximately between 1 and $1.5 \%$, as estimated by experienced administrative staff members from the billing deparment.

\section{* Clinical Data.}

The data colection has been performed by two physicians. For the interpretation of ECG data they were instructed by a cardiologist. But in case of doubt, scoring difficulties or if contradictory results were observed by the physicians comparing their interpretation and the results as described in the medical record, an experienced cardiologist was always consulted for a second opinion. In the latter case the opinion of the cardiologist was always followed. Some of the information, like the results of echocardiograms and heart catheterizations, was obtained fron the reports of the cardiologist(s). The intra-rater or inter-rater agrecment in the inierpretation of these tests had not been investigated. So the data reliability may be influenced by the accuracy of the interpretation made by these cardiologists.

To measure the accuracy of data collection (interpretation and transcription), an aselectly drawn subsample of 17 patients was independently re-scored by one physician.

Data scoring on the patient's background characteristics was found to be consistent in 96.5 $\%$ of the items; deviations occurred in the scoring of pre-stay angina pectoris, pre-stay coronary insufficiency and pre-stay hypertension.

In the demacation of time periods T0 to T3 one case was not consistent: one patient was scored by the first rating as having 'missing" values in time periods T0 and T1, while according to the second rating this patient did in fact have scores in T0 and TI. Closer examination revealed that this patient had a subacute infarction and as a result of this it was unclear how to demarcate the correct time periods. Consequently, there was a systematic misclassification in all items for this case. So it had to be excluded when determining the reliability of the data.

The agreement in the scoring of items related to physical examination (ischemic pain, palpitations, dyspnea, blood pressure etc.) was lowest in the first six hours of hospital admission, but improved in the other time periods (percentage of agreement in T0: $92.2 \%$; in T1: $94.8 \%$; in T2: $95.8 \%$; in T3: $97.1 \%$ ). Disagreement of scores mostly occurred for the duration of ischemic pain before hospital admission and the presence or absence of ischemic pain during stay.

The reliability of ECG-interpretation from the medical records of patients differed according to variables type. Agreement in the scoring of myocardial necrosis in the wall segments (by location of Q-waves) was 96.2 . \% in $\mathrm{T} 0,93.7 \%$ in $\mathrm{T} 1,92.5 \%$ in $\mathrm{T} 2$ and $100 \%$ in $\mathrm{T} 3$. Agreement in scoring of myocardial ischemia (by location of ST-segment changes) was somewhat lower $(92.7 \%$ in T0, $91.7 \%$ in T1, $87.5 \%$ in $\mathrm{T} 2$ and $98.0 \%$ in T3). Deviations occurred in the exact location of myocardial necrosis and ischemia in each wall segment.

Agreement in the scoring of rnythm disorders was $93.0 \%$ in $\mathrm{T} 0,95.8 \%$ in $\mathrm{T} 1,97.9 \%$ in $\mathrm{T} 2$ and $99.3 \%$ in T3. Differences appeared to exist in the presence or absence of sinustachycardia and ventricular escape rhythm. The agreement in scoring of conduction disorders proved to be: higher than that of rhythm disorders; $98.8 \%$ in T0, $96.6 \%$ in $\mathrm{T} 1$; $99.4 \%$ in $\mathrm{T} 2$ and $98.9 \%$ in T3. 
Agreement in scores of coronary stenosis measured by heart catheterization was $96.9 \%$ in $\mathrm{T} 0,93.7 \%$ in $\mathrm{T} 1,100 \%$ in T2 and $94.2 \%$ in T3. Differences occurred in the scoring of stenosis before and after PTCA and in one patient the time of catheterization was inconsistent: in one case catheterization took place in period $\mathrm{TO}$ and in the other case in period T1.

Agrecment in the scoring of the echocardiography data was almost total. The only difference was that in one case the echocardiogram data belonging to one patient was reported to be missing, while in the other case these data were not missing. This difference may be due to the fact that in one case the data of the echocardiogram were not found in the patient's medical record, but in records from the heart function department where echocardiography normally takes place. Strictly speaking, misclassification appeared to occur in about $7 \%$ of the cases.

Agreement in the scoring of enzyme values and the time it takes to reach an enzyme peak was $93.7 \%$ in $\mathrm{T} 0 . \mathrm{T} 1$ and $\mathrm{T} 2$, and $96.1 \%$ in $\mathrm{T} 3$.

Differences did occur in the registration of the highest enzyme peak within a time period and in some cases the number of hours after which a peak was reached was different. Sometimes these problems were caused by differences in demarcating the hospital time periods.

Agreement in the scoring of variables of the exercise ECG according to the Bruce protocol was $100 \%$ in T1, $90.6 \%$ in T2 and $100 \%$ in T3.

Differences in $\mathrm{T} 2$ existed because the data of one patient were reported to be missing in one case, while in the other case these were present. The same explanation can be given as for the scoring of echocardiography variables.

Agreement in the scoring of the thallium test could not he investigated. For all patients who had a thallium test, an independent scoring of results was performed by a single cardiologist specialized in interpreting thallium tests results. These scores were used in the data analysis.

The agreement in the scoring of medical interventions (i.e. thrombolytic therapy. PTCA etc.) that were performed during the various hospital time periods was $97.9 \%$ in $\mathrm{T} 0.98 .9 \%$ in $\mathrm{T} 1.100 \%$ in $\mathrm{T} 2$ and $100 \%$ in $\mathrm{T} 3$

The agreement in the scoring of types and amount of drugs administered during each time period was $96.2 \%$ in T0. $95.6 \%$ in T1. 95.() \% in T2 and $99.4 \%$ in T3. Differences in scoring did exist concerning the exact number of daily doses given within a time period.

The reliability of our data was the lowest for the interpretation of ECG data. Other inacuracies in scoring were related to the demarcation of the hospital time periods and might be caused by differences in rounding off days of stay when defining the hospital time periods.

Taking into consideration the retrospective character of the data collection and assuming that the intra-rater and inter-rater agreement is high when test results were interpreted by cardiologists, it is expected that the reliability of the data -as far as it could be establishedwill be sufficient for the purpose of this study. 


\section{Medical care costs and severity of illness: definitions and operationalization.}

In this chapter the methodology of defining and operationalizing the research concepts will be described. Attention will be paid to the measurement of costs of medical care in hospitals (5.1.) and on the measurement of the severity of illness of patients with myocardial infarction (5.2).

\subsection{Costs of medical care in hospitals.}

Costs of medical care are assessed, monitored and reported by means of specific accounting systems used in the financial-administrative departments of hospitals. Economists generally distinguish between costs made at ancillary departments (laboratories, operating rooms etc.), costs made in nursing departments or medical departments (for instance cardiology. gynaecology, internal medicine), and costs made by overhead departments (management, financial administration etc.). The costs of each hospilal depariment (labor, materials, equipment) will be calculated and (if defined) output measures are determined (number and type of services, number of patient days, etc.). However, most accounting systems do not provide adequate information about the actual costs of the total of medical care delivered to a patient during his or her stay at the hospital.

Fetter and Freeman (1986) follow a more generalized approach to this problem by stating that a hospital produces specific goods and services for its patients. These include "for example, X-rays, drugs, and laboratory tests ordered by physicians, as well as nursing care, operating room facilities and certain hotel and social services. However, since the real business of the hospital is to treat individual patients, these are only 'intermediate products'. The specific set of 'intermediate products" provided for each patient results in the "Tinal product' of the hospital (see figure 5.1).

Figure 5.1. Defining the hospital product.

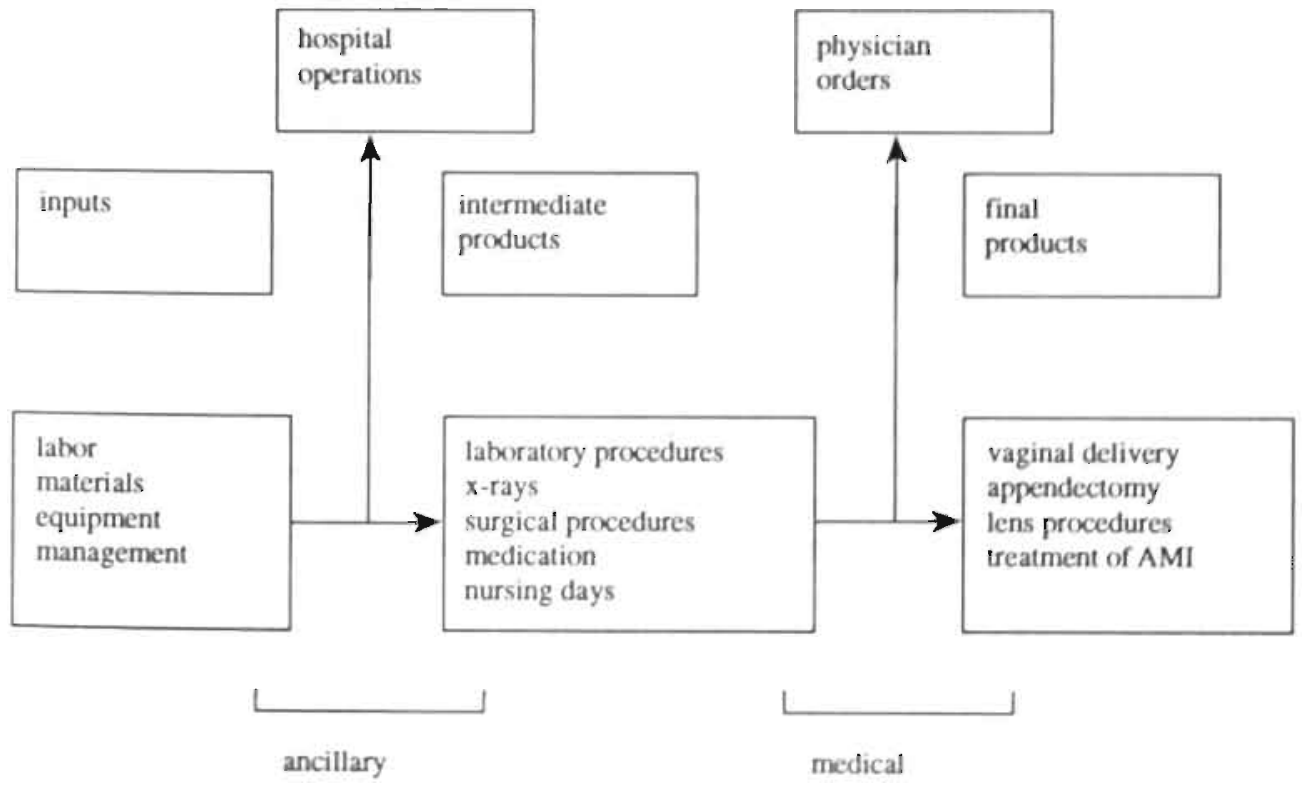


Assessing the costs of the intermediate products is an essential step in measuring the costs of the final hospital product (the total of medical care delivered to a patient). The costs per service multipiied by the number of services, added up for al! services are supposed to give the most reliable estimation of the real costs made during a patient's hospital stay. However, because of the complexity of calculation methods and the enormous variety in different services which a hospital may offer, the real costs for each hospital service are generally not available. For example, in a university hospital more than $600 \times$ different services can be distinguished. Assessing the real costs of all individual services may prove to be a goa! which is difficult to attain. As a substitute for the real costs per service, charges (tarifís) or specific costs weights may be used. However, this may affect the reliability of the determination of costs. For instance, the charges may not cover the real sum of money spent by the hospital to perform a service. In Dutch Health Care the charges per service are established at a national level by a uniform tariff system. Charges may not be exactly representative of the real cosis which may vary from hospital to hospital.

So, to determine the hospital costs per patient it is obvious that the real costs per service need to be assessed. These efforts towards an approximative calculation of the costs of medical services are being made at the University Hospital Maastricht.

The real costs per service have been estimated through extensive cost allocation studies carried out at the ancillary department level. Cost allocation studies have been performed at the elinical chemistry department (Limpens, 1986), bacteriology, virology and immunology department (Limpens, 1988; Roberts, 1989), haematology department (v.d. Kar, 1989), the heart function laboratory (Limpens, 1989), the iung funciion laboratory (Limpens, 1989), radiology department (Jetten, 1987), nuclear medicine (Wenemoser, 1988), operating room inclusive sterilization and recovery (Roberts, Kamm,1990), clinical pharmacy department (Brouwers, 1988; Hupperichs, 1988), physical therapy department (Steenbakkers, 1988), pathology department (Maes, 1987), gastro-enterology department (Schelling, 1988). hemodynamic laboratory (Coelen, 1988), department for tissue characterization (Roberts, 1989), blood transfusion department (Kamm, 1989) and the department of neurophysiology (Slabbers, 1987).

The cost allocation process is restricted to those hospital departments delivering services directly related to patient care. Indirect costs like general administration, maintenance, education and research either cannot be allocated to patient care or can only be allocated using arbitrary allocation-keys. These costs are therefore excluded from the cost allocation process.

In cost allocation studies the departmental costs (the inputs) are allocated to the various services performed by the department (the intermediate products). For each service cost prices are assessed. The methodology used in this allocation process will be further outlined in paragraph $5.1,1$.

The set of given services may differ by type and amount for each individual patient. The; use of hospital resources can be determined for each paient from information in the bill abstract. It is assumed that cost prices per service combined with the information derived from the bill abstract add up to a reliable measure of the real costs of medical care per patient. Results, from an application of this method for the patient population in question are described in paragraph 6.1. 


\subsubsection{Cost allocation of departmental costs to medical care services.}

In the cost allocation process the following definitions can be made (Limpens, 1986):

\section{* Indirect and direct services for patient care.}

Indirect services do not have any direct relation to patients but they do support patient care departments and the hospital in its totality in performing the care process adequately (for instance cleaning personnel or the goods and services of technical equipment suppliers). Direct services are those services which can be directly ascribed to the process of patient care (laboratory tests, X-ray's, surgical procedures, etc.). These are defined as the products of the ancillary departments.

\section{* Product related and non-product related costs.}

Costs of direct services can be specified into product and non-product related costs. If there is a direct relation between the departmental costs and a part of the production, these are called product related costs or specific costs. If the direct relation is not present we will speak of non-product related costs or general costs.

\section{* Components in Cost Allocation.}

In the cost allocation process four different components can be distinguished:

1. overhead costs: costs of management, administration and inventory;

2. capital related costs: costs of machines and instruments;

3. material costs: costs of materials and means;

4. personnel costs: costs of manpower.

\section{* Fixed and Variable Costs.}

During a period of time fixed costs can be regarded as independent of the size of production while variable cost may be changing with the level of production in the same period.

Figure 5.2. General process of cost allocation of departmental costs to medical care services.

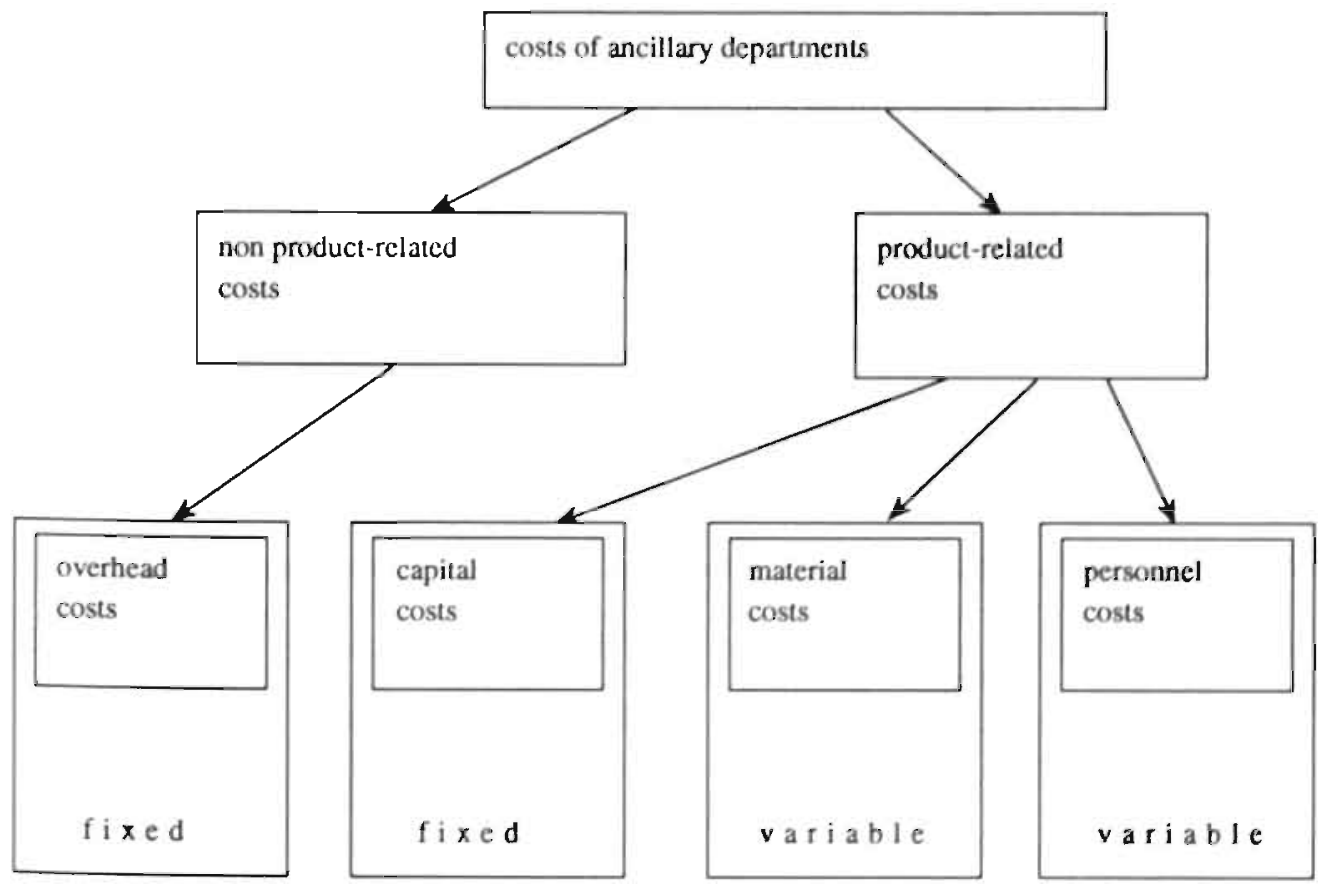


The main objective of the cost allocation process is to estimate a reliable cosi price for each service, keeping in mind the operating costs of the department in charge.

The mix of services will vary per department; some departments only perform a restricted number of different services "while others offer a large diversity of services. In addition, high and low frequency services occur in both situations. Consequently, establishing a reliable cost price for all individual services is a goal difficult to attain, especially if cost prices need to be revised or kept up to date.

In the development of the cost allocation system for hospital services at the University Hospital Maastricht some general principles have been applied (Limpens, Roberts 1988). Cost-allocation and the establishment of a cost-price per service are made on an annual basis. Cost allocation includes personnel, material, capital (depreciation, interest and maintenance) and overhead costs. A distinction is made between product related and nonproduct related costs. Costs per service are calculated in such a way that number of services times the costs per service equals the operating costs. For ancillary departments with a widespread mix of services, a distinction is made between (1) routine services for which cost prices are calculated separately for each service, and (2) non-routine services for which cost prices are derived from costs of routine services. Although the cost prices of non-routine services may be somewhat less reliable, it is assumed that the overall effect may be neglected, since routine services ( $20 \%$ of the service mix) generate the majority of costs ( 80 \% of the department costs). No distinction is made between urgent and non-urgent services. In addition, no distinction is made between services produced within or outside regular working hours. Thirdly, no distinction is made between services for patient care, research or cducation.

\section{Product related personnel costs.}

Allocation of product related personnel costs to the various services and the establishment of a cost price per service will be illustrated by a fictive example (table 5.3). A distinction will be made between routine and non-routine services. For routine services the average time in minutes spent by each category of personnel (analysts, assistants, technicians, physicians) is established through empirical studies. The average time (AT) of a service comprises the duration of all activities necessary to complete the service. For non-routine services the average time per service is assumed to be equal to the average time calculated over all routine services. It is supposed to be equal for all types of non-routine services (see example table 5.3): AT (non-routine services) $=\{(10 * 6)+(20 * 3)+(30 * 6)+(40 * 3)\} /(10+20+30$ $+40)=4.2$.

Multiplication of the total Number of services (Ns) from both routine and non-routine services by the Average Time per service will give the Total Time (TT) spent by a member of the personnel to perform certain services.

The Workload Proportion (WP) is defined as the relative weight in personnel costs of a service; this comprises the total time needed by a person to perform that type of service divided by the performer related 'total' time for all services ( 483 minutes in the example).

For instance, the Workload Proportion for service 'a' can be represented as:

$$
w_{p_{a}}=\frac{N a * A T a}{\sum N s * A T s}=\frac{10 * 6}{483}=0.12
$$


The service related Salary Costs (SC) are defined as the Workload Proportion (WP) multiplied by the Total Salary Costs (TS) of the performer (for the example set on $\mathrm{f} .1 .000,-$ for 483 time units).

The service related Salary Costs for service ' $\mathrm{a}$ ' may be written as:

$$
S C_{a}=\frac{N a_{* A T a * T S}}{\sum N s_{*} A T s}=\frac{10 * 6 * 1000}{483}=124.22
$$

The Cost Price of Personnel Costs (CPP) for each service may be calculated as service related Salary Costs (CS) divided by the number of services (Ns) a performer produces and can be seen as the estimated personnel costs of the service. So, the cost price of personnel costs for service ' $\mathrm{a}$ ' may be written as:

$$
C P P_{a}=\frac{1}{N a}\left(\frac{N a * A T a * T S}{\sum N s * A T s}\right)=\frac{A T a * T S}{\sum N s * A T s}=\frac{6 * 1000}{483}=12.42
$$

Table 5.3. Fictive example of cost allocation of product related persomel costs for routine and non-routine services in Dutch Guilders.

Total Salary costs (TS) of analyst have been set on $f 1000$.

$\begin{array}{rrrrrrr}\text { Service Number Average } & \text { Total Workload } & \text { Salary } & \text { Cost } \\ & & \text { Time } & \text { Time } & \text { Proport. } & \text { Costs } & \text { Price } \\ \text { (s) } & \text { (Ns) } & \text { (ATs) } & \text { (TTS) } & \text { (WPs) } & \text { (SCs) Personel } \\ & & & & & & \text { (CPPs) }\end{array}$

\begin{tabular}{|c|c|c|c|c|c|c|c|}
\hline \multirow{3}{*}{$\begin{array}{l}\text { Non- } \\
\text { Routine }\end{array}$} & $e$ & 5 & & & & & \\
\hline & $f$ & 5 & 42 & 63 & 0.13 & л. 130.43 & 77.8 .70 \\
\hline & $g$ & 5 & & & & & \\
\hline Total & & 115 & & $483^{*}$ & 1.00 & n 1000 . & \\
\hline
\end{tabular}

$\begin{array}{rrrrrrrr}\text { Routine } & a & 10 & 6 & 60 & 0.12 & f l .124 .22 & f 1.12 .42 \\ & b & 20 & 3 & 60 & 0.12 & f l .124 .22 & f .6 .21 \\ & c & 30 & 6 & 180 & 0.37 & f .372 .67 & f .12 .42 \\ & d & 40 & 3 & 120 & 0.24 & f .248 .45 & f .6 .21\end{array}$

* Performer related 'total' time for all services. 


\section{Product related material costs.}

Allocation of production-related material costs and calculation of a cost price per service will also be presented by a fictive example (see table 5.4.). The material costs per service are the average material costs to perform a service given the machinery setup. These include all types of material necessary to complete a service. The average material costs of routine services have been estimated using specific expertise of performing personnel and by independent observers. Costs of product related materials, which can not directly be allocated to a type of service, are equally divided over routine care services. The average material costs of non-routine services are derived from the average costs of routine services (see example table 5.4). Calculation of a cost price for material costs per service is made following the same rules as for personnel costs.

Table 5.4. Fictive example of costs allocation of product related material costs for routine and non-routine services in Dutch Guilders.

Total Material Cost (TMC) has been set on $f 2000_{2}$.

\begin{tabular}{|c|c|c|c|c|c|c|c|}
\hline & ervice & Number & $\begin{array}{r}\text { Average } \\
\text { Material } \\
\text { Cost } \\
\text { (MCs) }\end{array}$ & $\begin{array}{l}\text { Total } \\
\text { Cost } \\
\text { (TCs) }\end{array}$ & $\begin{array}{r}\text { Material } \\
\text { load } \\
\text { Proport. } \\
\text { (MPs) }\end{array}$ & $\begin{array}{r}\text { Material } \\
\text { Cosis } \\
\text { (MCs) }\end{array}$ & $\begin{array}{r}\text { Cost } \\
\text { Price } \\
\text { Material } \\
\text { (CPMs) }\end{array}$ \\
\hline Ronutine & $\begin{array}{l}a \\
b \\
c \\
d\end{array}$ & $\begin{array}{l}10 \\
20 \\
30 \\
40\end{array}$ & $\begin{array}{r}\text { fl. } 10.00 \\
\text { fl. } 50.00 \\
\text { f1. } 5.00 \\
\text { fl. } 25.00\end{array}$ & $\begin{array}{r}\text { f. } 100 \\
\text { f. } 1000 \\
\text { fl. } 150 \\
\text { ת. } 1000\end{array}$ & $\begin{array}{l}0.03 \\
0.39 \\
0.06 \\
0.39\end{array}$ & $\begin{array}{l}\text { fl. } 77.29 \\
\text { fl. } 772.94 \\
\text { f. } 115.94 \\
\text { f. } 772.94\end{array}$ & $\begin{array}{r}\text { fl. } 7.73 \\
\text { fl. } 38.65 \\
\text { f. } 3.87 \\
\text { fl. } 19.32\end{array}$ \\
\hline $\begin{array}{l}\text { Non- } \\
\text { Routine }\end{array}$ & $\begin{array}{l}e \\
f \\
g\end{array}$ & $\begin{array}{l}5 \\
5 \\
5\end{array}$ & fl. 22.50 & fl. 337.5 & 0.13 & fi. 260.86 & f. 17.39 \\
\hline Tota! & & 115 & & ff. 2587.5 & 1.00 & ค. 2000 ,- & \\
\hline
\end{tabular}

\section{Product related capital cost}

Capital costs refer to the equipment (machinery and hardware) used to perform a service. Allocation of product related capital costs to the various seivices and the calculation of a cost price per service is made by applying the same rules used for determining personnel and material costs. Routine and non-routine services are calculated in different ways. For routine services the average use, in minutes, of the equipment per type of service is determined by independent observers. The estimated use of equipment for non-routine services is derived from the average use of routine services. Costs of equipment which ean not be allocated to a specific type of service are equally divided over routine services. If more than one type of machinery is used to perform a service, a cost-price is calculated for each type of machinery and subsequently added for all machinery to obtain an overall cosi-price per service. 


\section{Overhead costs}

Overhead costs include all costs which are not allocated to the production related personnel, material and capital costs. These consist of:

personnel costs: management, secretarial offices and administration offices of departments:

- material cosis: office materials, office machinery and equipment and furniture. travelling expenses, xerox use, literature etc.

- capital costs: computers for administrative purposes.

The total overhead costs are equally divided over routine and non-routine services. Therefore the overhead costs consist of a uniform fixed amount for each type of service.

\subsection{Severity of Illness.}

Severity of illness is a familiar and often used term in patient care. Yet, little has been done to define, operationalize and measure this difficult and complex concept in a reliable and valid way. Studies in this research field have not ye! succeeded in overcoming the lack of 'golden' standards in the measurement of a patient's severity of illness. Severity of illness is a concept which cannot be measured entirely on its own; medically speaking il will always be associated with a patient's type of medical deficiencies and, in a more general way, with the type of diagnosis. Nonetheless, a more formal definition is necessary to delineate it.

The severity of illness of a patient within a clinically homogeneous diagnosis category is defined as the degree of pathologic deviation from the "normal' state of health measured by structural and functional medical deficiencies which belong to this diagnosis category. A diagnosis category is defined as a set (or syndrome) of medical deficiencies or complications that intrinsically belong to each other.

The structural and functional medical deficiencies may include clinical and non-clinical concepts (indisposition, malfunctioning in daily life or quality of life). In this study we will restrict ourselves to clinical concepts of severity of illness.

In the coming paragraphs an attempt will be made to operationalize the concept for patients with Acute Myocardial Infarction (AMI). A general and formal terminology will be developed and be used in chapter 6 to operationalize and measure severity of illness in AMI patients. In this chapter, some preliminary concepts and assumptions in defining Severity of Illness will be given. The difference between structural and functional deficiencies will be outlined. In addition, the difference between units of observation and characteristics of units of observation will be given. The assumptions and terminology will be then specified for AMI patients (5.2.1.). Next, a short overview of the relations that exist between the medical deficiencies will be presented using a causal model. In doing so a distinction will be made between:

* the severity of deficiencies causing AMI;

* the severity of the AMI deficiencies themselves;

* the severity of deficiencies caused by AMI.

Some methodologic considerations on the use of techniques of observation (ECG, echocardiograms, etc.) pertaining to unidimensional and multidimensional severity scales will be presented (5.2.2.). A measurement approach is proposed consisting of three interrelated elementary scales of Severity: the Intensity Scale, the Extent Scale and the Location Scale (5.2.3.). In the next paragraph it will be described how the principles above are used to determine the severity of illness (and changes therein) in AMI patients during their hospital stay. Some rules for categorizing patients into dynamic Severity Illness Categories will be given (5.2.4). Finally a summary will conclude this chapter (5.2.5). 


\subsubsection{Assumptions and terminology regarding severity of illness in AMI patients.}

\section{* Structural versus Functional deficiencies.}

A distinction can be made between medical deficiencies of a more 'structural' and a more 'functional' nature. 'Structural' medical deficiencies can be ascribed to organic subunits of the human body like the heart muscle or' coronary artery system. 'Functional' medical deficiencies can be ascribed to the heart and specific organic processes taking place within and between organic subunits. These processes fulfill certain vital needs of the organism, like the coronary oxygen supply to the heart by the coronary vessels or the cardiac output capacity of the cardiac ventricle; survival of the whole organism (and its organic subunits) is at stake, if these processes (slowly) degenerate or come to a stop.

\section{* Units of observation and Characteristics of units of observation.}

From the definitions above, some general methodologic rules can be derived. A distinction can be made between (1) units of observation and (2) characteristics of units of observation. The principal unit of observation in our study is the patient with Acute Myocardial Infarction. Structural units and functional processes within the heart first will be specified. The structural units of observation of the heart relevant for the purpose of measuring Severity of lilness are listed in scheme 5.5. The heart can be clinically subdivided into four main parts (units): (a) the left ventricle (b) the coronary artery system (c) the conduction system and $(d)$ the cardiac pacemaker. As the second row of the scheme illustrates, the four structural units of the heart are built up of smaller subunits: the left ventricle consists of wall segments, the coronary artery system of arteries, the conduction system of conduction fibers and the cardiac pacemaker of automatic fibers. The pathological characteristics of these subunits relevant for defining severity of illness are named the structural cardiovascular and cardiac deficiencies. These are mentioned in the third row of the scheme. Wall segments of the left ventricle may show myocardial ischemia or myocardial necrosis. Within the arteries, arteriosclerosis or coronary stenosis might be present. Structural medical deficiencies within conduction or automatic fibers are considered to be less relevant in AMI patients, but may be important in other diagnostic groups.

The functional processes within the heart can also be seen as units of observation. Five types of functional processes may be discemed within the heart, relevant for AMI patients. Left ventricle contraction, coronary artery perfusion, conduction as a process, rhythm behavior and cardiac function are the relevant processes which may show pathological characteristics. These pathological characteristics are entered in the last row of the scheme. The functional deliciencies are called respectively: abnormal wall motion, hypoperfusion of the coronary arteries (combined or not combined with insufficiency of the collateral circulation), cardiac conduction disturbances. cardiac arrhythmia and heari failure. 
Scheme 5.5. Structural units of observation and functional processes within the heart and their medical deficiencies.

\begin{tabular}{|c|c|c|c|c|c|}
\hline structural unit & Ieft ventricie & $\begin{array}{l}\text { coronary } \\
\text { system }\end{array}$ & $\begin{array}{l}\text { conduction } \\
\text { system }\end{array}$ & $\begin{array}{l}\text { cardiac } \\
\text { pacemaker }\end{array}$ & \\
\hline $\begin{array}{l}\text { structural } \\
\text { subunit }\end{array}$ & $\begin{array}{l}\text { I.v. wall } \\
\text { segments }\end{array}$ & $\begin{array}{l}\text { coronary } \\
\text { artery }\end{array}$ & $\begin{array}{l}\text { conduction: } \\
\text { fibcr }\end{array}$ & $\begin{array}{l}\text { (automatic) } \\
\text { fibers. }\end{array}$ & \\
\hline $\begin{array}{l}\text { characteristic of } \\
\text { (sub) unit }\end{array}$ & $\begin{array}{l}\text { myocardial } \\
\text { ischemia; } \\
\text { myocardial } \\
\text { necrosis }\end{array}$ & $\begin{array}{l}\text { artenosclerosis: } \\
\text { coronary } \\
\text { stenosis }\end{array}$ & - & - & - \\
\hline $\begin{array}{l}\text { functional } \\
\text { process unit }\end{array}$ & $\begin{array}{l}\text { Ief ventriculat } \\
\text { contraction }\end{array}$ & $\begin{array}{l}\text { coronary } \\
\text { artery } \\
\text { perfusion }\end{array}$ & conduction & $\begin{array}{l}\text { thythm } \\
\text { behavior }\end{array}$ & $\begin{array}{l}\text { cardiac } \\
\text { function }\end{array}$ \\
\hline $\begin{array}{l}\text { charactenstic } \\
\text { of functional } \\
\text { process unit }\end{array}$ & $\begin{array}{l}\text { abnormal } \\
\text { wall motion }\end{array}$ & $\begin{array}{l}\text { hypoperfusion } \\
\text { of arteries: } \\
\text { insufficiency } \\
\text { of collateral } \\
\text { circulation }\end{array}$ & $\begin{array}{l}\text { cardiac } \\
\text { conduction } \\
\text { disturtances }\end{array}$ & $\begin{array}{l}\text { cardiac } \\
\text { arrhythmia }\end{array}$ & hcart fallure \\
\hline
\end{tabular}

\subsubsection{Severity of Acute Myocardial Infarction: a causal model.}

Subsequently, the relationships hetween the medical deficiencies observed in patients with Acute Myocardial Infarction must be considered. By delining causes and consequences of the characteristics mentioned above one can hypothesize a causal model. This model can explain changes in dependent deficiencies, given particular changes in other deficiencies.

The general idea behind the causal model is that structural deficiencies will influence functional deficiencies, and not the other way afound. The relations between structural deficiencies can be regarded as a causal chain model (see diagram 5,6.): coronary arteriosclerosis (sometimes combined with coronary spasm) may lead to coronary stenosis. Coronary stenosis in turn may lead to myocardial ischemia. When this process of myocardial ischemia becomes irreversible this may lead to myocardial necrosis. Diagram 5.6. also visualizes the relations between myocardial ischemia and necrosis and the functional cardiac deficiencies; all direct relations between coronary stenosis and the functional deficiencies are supposed to 'pass' through the processes of myocardial ischemia and myocardial necrosis. Myocardial ischemia and necrosis can cause functional deficiencies of the heart (abnormal wall motion and-indirectly-heart failure). At the same time it can directly lead to specific functional complications (rhythm and conduction disorders). In their tum these complications may also lead to heart failure. Heart failure may cause several body deficiencies in other organs than the heart (lungs, kidney, liver, etc.). 
Diagram 5.6. Causal Model specifying relations between deficiencies in patients with Acute Myocardial Infarction.

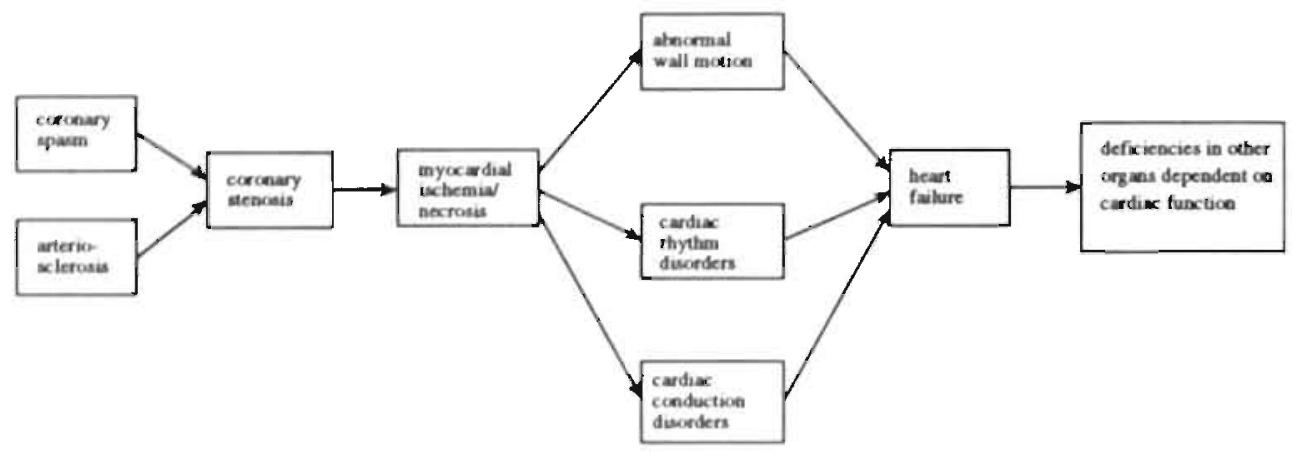

If one wishes to use the causal model presented in diagram 5.6. as a guideline for multivariate analysis in empirical research, its concepts must certainly be further specified into indicators. For example. 'Myocardial Necrosis' may be measured by means of three indicators, namely by the appearance of significant Q-waves in ECG leads, by certain biochemical changes in the blood serum, and by certain radiotopic changes observed in the myocard, when a so-called thallium test is performed. Patients who show significant Qwaves in ECG, who have these characteristic changes in their blood serum, and who show these thallium-negative areas in their myocard will be labeled as: 'having an Acute Infarction'. Patients who have no significant Q-waves, and no changes in blood serum, and no thallium-negative areas in the myocard will be labelled as 'Normal'. Patients with contradictory symptoms (Q-waves in ECG, but no blood serum changes, or: no Q-waves in ECG, but significant thallium changes) will be placed into 'in between' categories (i.e. 'suspected AMI').

\subsubsection{Defining Severity of Illness: an operationalization.}

The patient's overall severity of illness may be determined by a combination of scores on medical deficiencies. The measurement of the severity of a deficiency can be very clemental: the dichotomous scale is a widely used method of assigning scores to a patient. If no deficiency is present the score is ' 0 ', if a deficiency is found the score is ' 1 '. However, when medical professionals talk about the severity of an illness or deficiency, as a 'real life'concept, they think in terms of gradation. Therefore, the use of an ordinal measurement scale with more than two scores is a more realistic approach to defining and measuring the severity of a deficiency. The degree to which the severity will vary can be operationalized into rank scores, and patients with the same rank score can be categorized as belonging to the same group. Of course, one of the aims of social-medical research must be to investigate if severity scales approach interval measurement, where the length of the distances between the (rank) seores can be ascertained. Until this goal will be reached, let us assurne that a polytomous ordinal rank scale can be applied to the concept of severity of a medical deficiency. For instance, one could assume that for a certain deficiency four stages or rank scores exist, and that the resulting scale would describe the deficiency in a valid and reliable way. The minimum rank score ('no deficiencies') could be set at ' 0 ', the category "low level of deficiency' could be set at rank score ' 1 ', the category 'moderate' could be set at ' 2 ', and the maximum rank score ('3') could be given to the category 'life threatening deficiency'. However, a single deficiency can be measured with the help of multiple indicators which are 
the outcome of various medical techniques. As we have seen in 5.2.2., 'myocardial necrosis' can be measured by certain ECG-abnormalities, by certain changes indicated by a thallium test and by certain biochemical changes in the blood serum of patients. In principle, 'myocardial necrosis' seems to be a multi-attribute concept, which can be measured as a multidimensional scale. Furthermore, a unit of observation in medical science can be analyzed into several subunits, and in many instances sub-subunits can be distinguished. Techniques of observation are being used that can screen certain deficiencies within an organ at a sometimes microscopical level. Later on in the causal process deficiencies in one subunit may cause other types of deficiencies in other subunits of the same organ (see diagram 5.6.). Thus, in the first place we must state which deficiency at what levell of observation we are interested in (as we have done in scheme 5.5). When several subunits can be discerned that are relevant for a more precise (and medically valid) measurement of the deficiency, the concept of severity becomes necessarily a complex, multidimensional one. Naturally, this will often generate a situation, where more than one indicator must be used for one specific deficiency.

Indicators for specific medical deficiencies may imply several dimensions of severity of illness. Three independently defined, but related dimensions may be discerned:

(I) the intensity of a deficiency

(2) the extent of a deficiency and

(3) the location of a deficiency.

When an indicator measuring the deficiency for a unit is used, the Intensity of a Deficiency can be defined as the total of observed deviations times the specific weights of the degree of the deficiency, which its subunits get ascribed. For instance, when we measure the intensity of abnormal wall motion in the left ventricle (see $\$ 6.2 .6$ ), we can give certain weighted scores to every cardiac wall segment, while at the same time the technique of e.g. echocardiography will indicate that certain cardiac wall motion abnormalities are or are not present. The deficiency to be measured (the process of abnormal wall motion) in the higher unit (the left ventricle) is measured by a single indicator (contraction behavior) at the observational level of a subunit (left ventricular wall segment). Each wall segment is given a numerical weight ranging from ' 0 ' (in this case Normokinctic Function) 10 ' 3 ' (in this case Dyskinetic Function): these weights are multiplied with the observed deviations to a score for each subunit and eventually all scores can be aggregated or added up a total score for the unit. The example above is a one concept-one indicator case. When the concept to be measured is indicated by several indicators (e.g. myocardial necrosis), a more complicated situation exists.

When an indicator measuring the deficiency is used for a unit the Extent of the Deficiency can be defined as the spread of the observed deviations scores of this indicator over the. subunits, irrespective of any weight given to the degree of the deficiency. Using the same example as before, we can measure the extent of abnormal wall motion in the left ventricle by giving a score of ' 1 ' to every wall segment that shows signs of abnormal contraction. A score of ' 0 ' is given to every wall segment that shows no abnormalities at all. These ' 0 ' - and ' $l$ '- scores of all wall segments are then totaled for the entire left ventricle. One can interpret this aggregated total value as the spread of any abnormal wall motion over the left ventricle: the resulting absolute number can be seen as the extent of abnormal wall motion in the left ventricle of a patient. 
When an indicator measuring the deficiency is used for a unit, the Location of a Deficiency can be defined as the total of observed deviations times the specific weights, expressing the relative vulnerability of its subunits. In the above example one can measure the location of abnormal wall motion of the left ventricle by giving a weight to specific vulnerable cardiac wall segments. These weights for vulnerable sites, in the left ventricle can be given independently of the intensity weights. For the chosen example, it means that abnormal wall motion in the anterosepial segment can be considered more severe than in the other segments.

Together; the Intensity . Extent and Location scales can be interpreted as a three-dimensional measure of the concept of severity of a medical deficiency. Each dimension is independently defined with regard to the other two.

In empirical research, situations may arise in which one or even two of the three dimensions of the measure collapse into a much simpler concept of severity of illness. For instance, when the deficiency indicator can only be measured by a dichotomous scale $(0-1)$, the intensity dimension disappears and a two-dimensional concept of severity of illness remains. This often happens, when the degree of measurement in research is rather crude. A further degeneration of the three dimensional measure may occur, if the unit and subunit of observation are one and the same: in this case the extent and location dimensions collapse into a single unidimensional scale. This applies for instance, for the measurement of cardiac conduction disorders: only a gradation measuring the type of conduction disorder can be defined and given weights for these disorders only the intensity scale remains. The most extreme degree of this degeneration in measurement occurs when the above restrictions are combined. In this case only a crude unidimensional score remains which can be used to register the absence of presence of the deficiency.

\subsubsection{Severity of AMI patients during hospital stay; changes in severity and rules to obtain dynamic Severity of Illness Categories.}

As we have seen in previous paragraphs, severity of illness for AMI patients may be ascertained by various cardiovasculat and cardiac deficiencies. Each deficiency can in turn be measured by one or more indicators and each indicatos can be expressed in terins of one or more severity dimensions. The patient's severity of illness must be seen as a dynamic concept. In general the severity of illness will change while a patient is in hospital.

To measure changes in severity of illness, the condition of a patient needs to be measured at various moments during hospital stay. For AMI patients, who generally experience a lifetheatening situation, these measuremenis should focus on the first part of their hospital stay, in which indices may show extreme variations. The later stay period can generally be described as an after-crisis situation. Based on these considerations, four time periods have been distinguished (see $\$ 3.3$ ). When severity of illness is measured repeatedly within these four periods certain changes in severity may be observed: patients may stabilize soon after crisis or at a later point, they may develop complications, or they may deviate consistently in some way (table 5.7). 
Table 5.7 Some patterns of changes in severity of illness.

\begin{tabular}{llllll} 
Pattern & TO & $T 1$ & $T 2$ & $T 3$ & Description \\
1 & deviating & normal & normal & normal & early stabilization \\
2 & deviating & deviating & normal & normal & stabilization after 3 days \\
3 & deviating & deviating & deviating & normal & stabilization after 10 days \\
4 & deviating & normal & deviating & normal & incidental complication \\
5 & deviating & deviating & deviating & deviating & consistently deviating \\
\hline
\end{tabular}

The various severity patterns which occur during a hospital stay may be measured for each medical deficiency belonging to the AMI diagnosis. For instance the severity of myocardial ischemia may be measured repeatedly by daily ECG. Severity patterns of myocardial ischemia during hospital stay which appear to be similar in a medical way may then be grouped into one severity category. Severity patterns may be seen as dynamic severity categories. Subsequently, the dynamic severity categories may be placed in a rank order of increasing severity. For instance, the severity pattern 1 in table 5.7. may be grouped into one dynamic severity category A. Severity patterns 2 and 3 into category B and 4 and 5 into two categories $C$ and $D$. A rank order in severity may be given in which it is assumed that category A is less severe than category B and category C is more severe than B, etc. In this process of categorizing and subsequent ranking medical specialists need to be consulted regularly.

The process of grouping severity patterns into dynamic severity categories and ranking the categories according to their severity may involve methodological problems. Conseguently, some additional rules need to be specified.

\section{(1) Problems involving indicarors measured only once during hospital stay.}

Evidently, severity indicators must be repeatedly measured during the patient's hospital stay in order to determine changes in severity. Therefore the clinical observation technique by which the indicators is being measured must be applied several times during a patient's hospital stay (and preferably once during each of the four time periods). Examples of such severity indicators are those which are based on results of ECG (which provides information about myocardial ischemia and necrosis plus rhythm and conduction disorders). If during a period more than one ECG is made for a patient, the one with the most deviating results from the 'normal' situation will be selected for classification.

However, some cardiac deficiencies are only measured once during the whole hospital stay. Examples of such deficiencies are cardiac wall motion and heart failure which are estimated by echocardiography. If an observation technique is applied only once, it will be impossible to categorize patients according to the changes in severity of this particular deficiency. Thus, next to the more 'dynamic' severity classification of patients, a more 'static' classification must be used which characterizes their illness condition during the whole stay. In chapter 6 , severity of illness for both the dynamic and static classification will be dealt with.

\section{(2) Problems in ranking categories of patients.}

In the above line of thought it has been assumed that the grouped patterns of a deficiency can be placed into a rank order of increasing severity. This ordinal scale construction will be used in the forthcoming data-analysis to explain differences in costs. However, there are 
situations in which severity patterns of a deficiency can not be placed into a single unidimensional rank order. For instance, cardiac rhythm disorders may be so diverse and may or may not be important at different moments during hospital stay, that forcing them into a unidimensiona! scale seems impossible at this time of research. We did not feel confident enough to hypothesize which underlying dimensions can be found within all these types of rhythm disorders. Furthermore, this problem should better be solved in a more medical (cardiological) research setting. For the time being our solution is a simple one: all patients are categorized into one uniquely defined nominal group and this nominal category will be used as a factor in the analysis of medical care costs.

\section{(3) Problems with missing values.}

A typical medical deficiency may not always be measured in all patients. For instance, the severity of coronary stenosis can only be measured for those patients who have had at least one heart catheterization during their hospital stay. For patients who have not undergone catheterization during their stay or for those patients, whose results of catheterization are lost, illegible or otherwise unknown, no information about the severity of coronary stenosis. can be transcribed. In this case, the grouping of patients into severity categories may be limited to lower numbers of patients.

Missing data in indicators of severity of illness may of course also result when patients are discharged early, are transferred to another medical department or if they die during hospital stay. This problem also arises when patients are admitted from another hospital during a later phase of their illness period (see $\$ 4.2$ ) In the eventual cost analysis some correction has to be made for patients who have these types of missing values for indicators. To overeome these difficulties, which may seriously narrow down the number of patients in data analysis, we will regard 'missing values' as a separate subcategory of patients in each analysis. Sometimes extra (dummy) variables are constructed for the sole purpose of supplementing the numbers of patients in the categories of severity of illness. In $\$ 7.1$. a precise description of the solution for this typical 'missing values' problem will be given.

\subsubsection{Summary.}

To measure a patient's severity of illness within a homogeneously defined diagnosis group a conceptual distinction must be made between (1) structural and functional medical deficiencies and (2) units of observation and characteristics of units of observation. Relations between the medical deficiencies may be hypothesized in a causal model. The severity of a medical deficiency may he measured with single or multiple indicators. It is assumed that three interrelated severity dimensions can be discerned: the intensity, the extent and the lexation of a deficiency. Sometimes all three dimensions can be distinguished in measuring severity of illness, sometimes they may collapse into one or two dimensions. The severity of a medical deficiency may he measured cither per time period or summarized for the whole hospital stay. In the latter case it will be condensed into dynamic severity categories. These may be obtained by defining various patterns of severity of illness. Patients having similar severity of illness patterns can be categorized within the same severity group.

The proposed methodology for measuring dynamic sexerity categories will be applied for the AMI patient population. Measurement of the severity of illness per time period and the determination of dynamic severity categories for each deficiency as outlined in the causal model of patients having acute myocardial infarction (diagram 5.6.) will be more extensively described in paragraph 6.2. 


\section{Measurement of research concepts.}

The methodology proposed in chapter 5 will be applied to operationalize and measure the research concepts outlined in the models of explanation (chapter 3.2.). The operationalization and measurement process is described for the patient population at hand: 464 patients with acute myocardial infarction.

This chapter will concentrate on operationalizing the three major research concepts:

- Cost of medical care (6.1)

- Severity of illness (6.2)

- Background characteristics (6.3)

\subsection{Costs of medical care.}

The costs of medical care for the patient population at hand are measured by (1) the type of service, (2) the number of services and (3) the cost-price of each service (see 5.1.1). For each patient, resource data have been collected from our hospital billing administration. Resource data consist of the number and type of services given to a particular patient during his or her hospital stay. In order to measure the exact costs of a hospitalized patient, ambulatory care services and services requested by physicians other than cardiologists are excluded. Results from cost price studies of medical care services have been used to measure the financial impact of the medical care delivered. Medical costs are calculated by multiplying the service-specific-cost-price by the amount of services performed. In our research strategy the service-specific-cost-price includes (1) product-related personnel costs ${ }^{2}$ and (2) product-related material costs. Product-related capital costs and overhead costs are not taken into account because of their fixed character. Cosis are limited to variable costs which are assumed to be dependent on characteristics of the patient population. Overhead and capital costs in hospital care are assumed to show less variations due to illness or patient characteristics. Patient characteristics may only influence these costs on a long term basis. For example, severely ill patients may require more laboratory tests. This will mainly affect costs of materials needed and time of the laboratory personnel, but in general this will have no direct impact on the costs of the machinery or the departmental administration. Our research strategy concentrates on explaining medical care cost differences on the hasis of specific illness and patient characteristics. Obviously only variable costs which are strongly related to illness and patient characteristics will be taken into account.

The only exception to the procedure described above is the calculation of the costs of drugs which are not recorded in the billing system; the types and amounts of drugs are derived from the patient's medical record and cost-prices are estimated on the basis of daily doses.

Costs of medical care pei patient will be divided over the four hospital time periods defined in our model of explanation (chapter 3.3). For some specified categories of services, costs made in time period T0 (first six hours of hospital admission) had to be included in T! because they are recorded per twenty-four hours.

Various hospital costs will be described in the following sections: costs of diagnostic services (6.1.1); costs of medical interventions (6.1.2); costs of nursing care (6.1.3) and other costs. like physical therapy (6.1.4). Within these four major groups of medical care more detailed costs categories will be defined on the basis of their medical relevancy to the hospital care process.

2 Physician salary costs have been exluded from the personnel cost-price because they đo not represent costs of ancillary departments. 


\subsubsection{Costs of diagnostic services.}

\section{* Costs of Laboratory Services.}

Laboratory services can be distinguished into routine and specific tests. Routine laboratory tests are tests that are normally performed irrespective of the specific illness of a patient. These services provide an overall impression of the patient,'s physiologic health condition. These include: general hematology tests (like hemoglobin " hematocrit, counts of leukocytes, crythrocytes, thrombocytes), general blood chemistry tests (like glucose, urea, creatinine, sodium, potassium) and general urine chemistry tests (like protein, glucose, urobilin). Specific laboratory tests include all extra tests considered necessary given the patient's health condition and/or discase. For the patient population at hand these include specific blood chemistry tests (like Creatine Phospho-Kinase (CPK), Serum Glutamic Oxalacetic Transaminase (SGOT, also known as ASAT), Serum Lactic Dehydrogenase (LDH), Serum Glutamic Pyruvatic Transaminase (SGPT, also known as ALAT), repetitive determination of daily glucose and urine acid levels), specific hematology tests (like reticulocyte, eosinophile couni, blood gases and hematologic coagulation) and other blood tests (like hormone tests and iests from microbiology and immunology). Costs of all (routine and specific) laboratory services for the patient population at hand are presented in appendix. 2. Per patient a mean number of 80.2 laboratory tests are made during the total hospital stay (mean costs: fl. 218.34). The greater part (75\%) of these tests takes place during the acute phases of the hospital stay (in time periods T(1 and T1).

\section{* Costs of Routine Diagnostic Services.}

Rouline diagnostic services are normally performed for each AMl patient. These involve ECG-registrations (appendix 3) and radiology tests (X-thorax) (appendix 4). When patients are admitted to the Coronary Care Unit, ECG tests are performed on a very regular basis and. are included in the costs of intensive care. For the patient population at hand, most patients spend their first days of stay in the intensive care ward. This may explain why the costs of ECG tests are relatively low during the first days of the hospital admission. On average, 7.5 ECG's are made per patient (mean costs: $\mathrm{fl} .47 .86$ ) and 2.1 radiology tests are performed per paticnt (mean costs: fl. 46.11).

\section{* Costs of Specific Diagnostic Services.}

Specific diagnostic services for AM! patients include all additional non-laboratory tests considered necessary given the patient's specific health condition and/or disease. Because of large differences in technique, in what is measured and number, these costs are distinguished into five categories.

\section{Heart Catheterization.}

During a heart catheterization several activities may take place. Normally a session consists of left catheterization with (1) pressure measurement and $\mathrm{O}_{2}$-saturation measurement, (2) left, ventriculography and (3) coronary arteriography. Next to these activities, less regular activities may occur, like right heart pressure measurement with Swan-Ganz catheters. Costs are calculated for every separate activity. For the costs of catheterization (appendix 5) the unit of measurement is the catheterization activity. A catheterization is generally done during the acute phase of the stay: in $54.5 \%$ of the patient population the catheterization occurs at the very beginning of the hospital admission in T-0 and 29.3 \& in T-1. Relatively few catheterizations are performed in the following two periods. 


\section{Holter Electrocardiogram or 24 hours ECG.}

This test is normally performed on some patients to detect (severe) rhythm and/or conduction disorders. The total costs for our patient population are relatively low (see appendix 7). This test is only performed 72 times in total (distributed equally over all time periods) which amounts to a mean of .16 tests per patient (mean costs: fl. 8.43).

\section{Echocardiography.}

In some cases echocardiography is performed in combination with a Doppler test to estimate cardiac valve function during the same session and using the same machinery. Costs of Doppler tests are also included under the heading 'costs for echocardiography' (see appendix 8). Echocardiography for AMI patients is normally performed three days after admission. In total 417 times a test is made (total costs: $\mathrm{fl}, 20,201.05$ ), which amounts to a mean of .90 tests per patient (mean costs: fl. 43.54).

\section{Exercise electrocardiogram using the Bruce Protocol.}

An exercise ECG is usually performed to detect post-myocardial infarction angina and is normally planned just before patients leave the hospital. Therefore, costs due to this test only occur in time periods T2 and T3. Costs of this test (appendix 6) are generally made beiween three and ten days after the admission. For the patient population at hand in total 206 Bruce protocols are applied which amounts to a mean of .44 tests per patient (mean costs 11.17 .20 ).

\section{Nuclear Cardiography.}

This category consists for the greater part of the so-called thallium test. During this test (wo conditions are (simultaneously) investigated normally: (1) thallium uptake at rest and (2) thallium uptake during exercise. In appendix 9 costs of nuclear cardiography are given. The number of services is based on the number of (research) activities during a session. Like the Bruce protocol, a thallium test is performed normally to measure post-myocardial infarction angina or to ascertain the magnitude of myocardial necrosis. In general this test is planned a few days before patients check out the hospital. This explains why costs of this test only occur in time period T2 and T3. In total 235 activities are performed which amounts to a mean of .51 per patient (mean costs: 1.37 .07 ).

\subsubsection{Costs of medical interventions.}

Medical interventions in myocardial infarction may be distinguished into three major types.

\section{* Drugs.}

Thrombolytic therapy is a relatively new and very effective treatment in cardiology. Administration of thrombolytic drugs within the first hours of the (suspected) infarction leads to reperfusion of totally occluded or severely stenotic coronary arteries. Because of their impact on the patient's survival severity of illness and the ensuing medical care costs, the costs of thrombolytic therapy are distinguished from costs of other cardiovascular medical drugs.

\section{Thrombolytic Therapy.}

During the time period under study several thrombolytic drugs were administered. However, the most common and most frequently used thrombolytic drug was 'streptokinase'. Other thrombolytic drugs such as 'APSAC', 'urokinase'. 'pro-urokinase', and 'rt-PA' were applied. less frequently. These other thrombolytic drugs may generally be considered a consequence of specific research protocols. Alternative drugs might also have been used in case of contra- 
indications to the use of streptokinase. Although the costs of the different types of thrombolytic drugs are varying considerably (streptokinase: fl. 557.55 and urokinase: fl. 1813.98 per dose), the costs of streptokinase are thought to be relevant for setting a costprice for thrombolytic therapy. This decision is made because streptokinase was the most frequently used drug. Because thrombolytic therapy is only successful if administered within about the first six hours of the infarction, almost all costs for thrombolytic therapy are made in T-0 (appendix 10). In total 150 thrombolytic therapies are given (almost one third of the patients).

\section{Other Drugs.}

Other drugs include Sympathoplegic Drugs, Nitrates, Calcium Antagonists, Diuretics, Glycosides, Anti-Arrhythmic Drugs, Sympathomimetic Drugs, Vasodilator Drugs, Drug treatment of hypertension. Anti-Coagulants and Acetylsalicylic Acid. The calculation of these costs is based on the cost price of daily doses for each type of drug therapy. The accumulated costs of these drugs are presented in appendix II. On average 38.2 daily doses of these drugs are administered per patient, amounting to a mean cost of fl. 71.60 per paticnt.

\section{* Percutaneous Transluminal Coronary Angioplasty (PTCA).}

PTCA is performed during the heart catheterization session. Costs of PTCA are calculated as extra-costs made on top of the costs of a heart catheterization. As illustrated in appendix 12, PTCA is performed most frequently during the acute phase of the myocardial infarction; that's why $60.9 \%$ of all PTCA applications are performed in T0. The cost-price of PTCA is 11. 3677.24 and the mean costs per patient are f1. 649.86 .

\section{* Other Medical Interventions.}

Other medical interventions relevant to this study include resuscitation, a temporary (external) pacing system and the use of an intra-aortic balloon pump. These types of interventions in patients are extremely rare and the cost price per intervention type tends to vary considerably. We have decided to regard the 'other medical interventions' as a rest category and have combined the costs (appendix 13).

\subsubsection{Costs of nursing care.}

Costs of nursing care are indicaled by the patient's length of stay. The mean length of stay per patient amounts 1011.45 days (std.dev: 9.60). Costs of nursing care at the Coronary Care Unit (CCU) are calculated separately. These costs are calculated on the basis of the total material and personnel costs of this department over the year 1988 divided by the total number of CCU days in that year. This results in the average costs per CCU-day. It is assumed that the average daily costs per type of patient do not differ significantly, because in mos! cases standard procedures will be applied in patients with AMI. For each patient the aciual number of $\mathrm{CCU}$-days is multiplied with the average costs. The costs of this intensive nursing care are described in appendix 14. The total number of days for the whole patient population can be divided into $45 \%$ of days at the coronary care unit and $55 \%$ of days at the medium care unit or the 'normal' care unit. The mean costs of CCU per patient amount to fl. 1392.02 (std.dev. 1577.40). 


\subsubsection{Other Costs.}

Other costs besides those of diagnostic tests, medical interventions and nursing care are costs for physical therapy. Costs of physical therapy are described in appendix 15. This type of care concerns the physical rehabilitation of patients. In total fl. 11,176.93 is spent, which amounts to a mean of fl. 24.09 per patient.

There may be other types of costs made during a patient's hospital stay that are not mentioned in one of the categories above. These are excluded from this study for lack of any direct relation to AMI. These costs are sometimes related to specific and less frequent problems involving consultation of other medical specialists, kidney dialysis etc. These services amount to less than one percent of the total amount of services. Another important cost category concerns physician salaries. These will however not be investigated in this study because at the time of investigation no adequate workload measures had been available and no allocation of time spent by physicians to patient care for the 464 AMI patients could be made. Although the total costs of a patient's hospital stay might be somewhat higher because some costs have been excluded, it is expected that the cost calculation and measurement is fairly representative for the patient population at hand.

\section{Summary.}

The costs of the patient population concerning the total hospital stay are summarized per category in table 6.1. As can be seen from this table costs may vary considerably per type of medical care. The mean costs per patient are relatively high for heart catheterization and PTCA. Costs of laboratory services and thrombolytic therapy are moderately high. Extremely high costs are made as a result of CCU-stay. In almost all categories a large variation in costs is observed. Explanations for these differences in costs of medical care and length of (CCU) stay will be given in chapter 7 .

Table 6.1 Cost of medical care in patients having Acute Myocardial Infarction ( $n=464)$.

\begin{tabular}{|c|c|c|c|c|c|}
\hline Variable & Description & $\begin{array}{l}\text { Number of } \\
\text { senvices }\end{array}$ & $\begin{array}{l}\text { Total } \\
\text { costs }\end{array}$ & $\begin{array}{l}\text { Mean costs } \\
\text { per patient }\end{array}$ & Sid Dev \\
\hline$C L A B$ & Costs of laboratory services & 37214 & $101,310.34$ & 218.34 & 183.16 \\
\hline CECG & Costs of ECG's & 3492 & $22,209.12$ & 47.86 & 42.60 \\
\hline CRADIO & Costs of radiology & 962 & $21,396.40$ & 46.11 & 46.17 \\
\hline CCATH & Costs of heart catheterization & 857 & $269,612.54$ & 581.06 & 750.17 \\
\hline CHOLTER & Cosis of holter ECG tests & 72 & $3,909.60$ & 8.43 & 25.34 \\
\hline CECHO & Costs of echocardiography & 417 & $20,201.05$ & 43.54 & 32.76 \\
\hline CBRUCE & Costs of Bruce ECG tests & 206 & $7,982.50$ & 17.20 & 20.58 \\
\hline CTHAL & Costs of thallium tesis & 235 & $17,200.68$ & 37.07 & 71.18 \\
\hline CTHROM & Costs of thrombolytic therapy & 150 & $83,632.50$ & 180.24 & 280.89 \\
\hline CDRUGS & Costs of drugs & 17709 & $33,223,40$ & 71.60 & 79.14 \\
\hline CPTCA & Costs of PTCA & 82 & 301.533 .68 & 649.86 & 1424.79 \\
\hline COTHER & Costs of other interventions & 95 & $14,631.44$ & 31.53 & 257.26 \\
\hline CPHYS & Costs of physical therapy & 1118 & $11,176.93$ & 24.09 & 30.32 \\
\hline $\operatorname{CCCU}$ & Costs of coronary care & 2228 & 645.897 .20 & 1392.02 & 1577.40 \\
\hline LOS & Length of stay (in days) & $531 !$ & $\longrightarrow$ & 11.45 & 9.60 \\
\hline
\end{tabular}




\subsection{Severity of Illness of patients having AMI.}

The patient's severity of illness was measured by observing the cardiovascular and cardiac deficiencies as outlined in the causal model for patients with Acute Myocardial infarction (see chapter 5.2.1). In defining the research concepts, background information was obtained and distracted from several standard works on cardiology (Fuster 1975, Gensini 1975, Plotnick 1982 "Braunwald 1984, Willems 1985, Factor 1986, Gorlin 1986, Hurst 1986). Furthermore the definitions and terminology used in this paragraph are derived from sessions with the panel of cardiologists. The different concepts that will be operationalized in the next paragraphs are:

Severity of coronary stenosis (6.2.1)

Severity of myocardial ischemia (6.2.2)

Severity of myocardial necrosis (6.2.3)

Severity of ischemic pain $(6.2 .4)$

Severity of cardiac wall motion abnormalities (6.2.5)

Severity of cardiac rhythm disorders (6.2.6)

Severity of cardiac conduction disorders (6.2.7)

Severity of heart failure (6.2.8)

Severity of physical deficiencies and specific cardiac complications (6.2.9)

We will summarize the main points of paragraphs 5.2.1. to 5.2.4. The severity of these cardiovascular and cardiac deficiencies is operationalized and measured by means of specific indicators. For each indicator a severity rank order was constructed. Sometimes a typical deficiency can be measured by a set of distinct indicators. For example, 'myocardial necrosis' can be measured by three indicators, namely: the appearance of significant Qwaves at the ECG, certain biochemical changes in the blood serum and certain changes of the uptake of nuclides in the myocardium at nuclear cardiography. In other situations a typical cardiac deficiency can be measured by one only indicator. For example "cardiac wall motion abnormalities' will be assessed using echocardiography. For deficiencies which can be measured by multiple indicators, a separate scale, comprising a severity rank order, was constructed and described for each indicator.

Sevcrity of a cardiovascular or cardiac deficiency can be secn as a property or characteristic of the heart as a whole, but it can also concern a part or a process within the heart (see chapter 5.2.1.). Thus, the unil of observation may be differentiated into several subunits. For example, the left ventricular wall (unit) consists of several wall segments (subunits). If several subunits can he discerned a more precise measurement of the deficiency can be made using the characteristics of all (relevant) subunits. Data collection of medical deficiencies was always performed on the lowest level of measurement.

For cach indicator measuring a certain cardiovascular or cardiac deficiency severity o[ illness is operationalized by three independently defined dimensions (see chapter 5.2.2.), namely:

* the intensity of the deficiency

* the extent of the deficiency

* the location of the deficiency

The dimensions in severity of an indicator are operationalized by attaching weights to the scores of cardiovascular or cardiac deficiencies. The weights form a rank order of increasing severity. The severity weights that were used were derived from professional judgements of three cardiologists; two from the University Hospital Maastricht and one from the Catharina Hospital Eindhoven. Independently from each other, all three cardiologists judged the 
severity of each deficiency. In case of discordance over the severity weights, each cardiologist was confronted with the weights of the other two. The severity weights were applied after consensus was reached between the three cardiologists.

For the patient population at hand severity-indices are constructed for each defined time period. However, during hospital stay a patient's severity of illness is subject to changes. Therefore, specific severity categories are established which will reflect alterations in severity during hospital stay. The severity categories are based on the results measured by the time specific severity indicators. (T0 to T3). If severity indices are measured repeatedly during the patient's hospital stay (like ischemia measured by ECG) severity categories are determined representing the severity trend with respect to the patient's total hospital stay. In case there was a large number of different severity categories, these will be categorized into smaller numbers. If severity indices are measured only once during the hospital stay (like wall motion abnormalities measured by the echocardiogram) no changes in severity can be measured. In this case the index measured is thought to be representative for the condition of the patient during whole hospital stay (see $\$ 5.2 .4$.).

\subsubsection{Severity of coronary stenosis.}

A stenosis in the coronary arteries is measured by means of coronary angiography. This is a diagnostic method involving injection of a X-ray sensitive dye into the blood. X-rays taken during and after the injection show the inner dimensions of the primary arteries and their branches, outlined by the contrast medium. This test visualizes and delineates coronary artery stenosis in terms of location, extent and degree of stenosis.

The severity of coronary stenosis depends amongst other things on the specific location of the stenoses in a coronary artery. Cardiologists usually restrict themselves to detect stenosis in specific parts of the artery and its branches. In the context of this study only stenoses in the three primary arteries are thought to be relevant. The three primary coronary arteries are: (1) the circumflex branch ( $\mathrm{RCX}$ ) which provides normally blood to the anterobasal, lateral, inferior and posterior wall of the left ventricle of the heart and in some cases to the AV-node. The RCX can be subdivided into five arterial segments: proximal, marginal branch, mid and distal, posterior lateral branch and posterior descendens branch.

(2) the left anterior descending branch (LAD) which is supplying normally blood to the septum and the anterobasal, anterior and apical wall of the left ventricle of the heart. The LAD can be subdivided into five arterial segments: proximal, mid, distal, first diagonal branch and second diagonal branch.

(3) the right coronary artery (RCA) which supplies generally blood to the sinus and AVnode, the right ventricular wall and a part of the inferior, posterior and apical wall of the left ventricle of the heart. The RCA can be divided into four arterial segments: proximal, mid, distal and posterior descendens branch.

The degree of stenosis for every arterial segment is recorded as a percentage of narrowing or obstruction varying from $50 \%$ stenosis to $100 \%$ stenosis (occlusion).

In acute situations, haemodynamically significant stenoses in the coronary system can be treated by thrombolytic drug therapy or Percutanous Transluminal Coronary Angioplasty (PTCA). These treatments are performed to reduce the degree of stenosis or to remove a specific occlusion in a coronary artery so that arterial blood flow will not be hindered anymore. Usually these interventions will reduce considerably the severity of stenosis.

If drug therapy was applied the last scores of coronary stenosis during the heart catheterization were taken. In case PTCA was performed two different scores were recorded: stenosis before and after PTCA. If no intervention (drug therapy or PTCA) has 
taken place, the last score of coronary stenosis during heart catheterization was recorded. If a stenosis was measured in the Left Main Coronary Artery the severity of this type of stenosis was recorded for both proximal LAD and proximal RCX.

The intensity of coronary stenosis.

First, the weights of the degree of the stenosis were determined separately for each arterial segment. Next, the degree of the stenosis with respect to each coronary artery and subsequently to the coronary artery system was calculated.

If there was not a significant degree of stenosis $(<70 \%)$ a severity weight of ' 0 ' was given. Patients got severity weight ' 1 ' when the degree of stenosis varied between 70 and $99 \%$. The highest severity weight of ' 2 ' was given in case of $100 \%$ stenosis (occlusion).

These weights were applied to measure the 'intensity of the stenosis' for each coronary arterial segment of RCA, LAD and RCX. Empirical combinations in weighted scores over arterial segments revealed that for every coronary artery the severity score of the stenosis did range from 0 'no significant stenosis' to 3 'combination of occlusion in one arterial segment and significant stenosis in another arterial segment'. The scores of the 'intensity of stenosis' per ariery (0-3) were again aggregated into one overall severity score by adding the individual stenosis $z$-scores of the three primary arteries (ranging from -1.00 thru 3.00). Stenosis $z$-scores were used because the number of arterial segments varies per primary artery. The sum of the $\mathbf{z}$-scores represents the degree of stenosis with respect to the coronary afiery system.

\section{Severity Categories.}

The severily categories were based on seores after PTCA was performed. Because PTCA is performed during the same catheicrization session the score afier PTCA was assumed to be relevant for measuring the severity of coronary stenosis. From the patient population at hand only 182 patients were catheterized ( 119 patients only once and 63 patients twice). Thus a actual changes in severity of the degree of coronary stenosis could only be measured for the 63 patients. For the remaining group of patients the single 'intensity' score was used. It is presumed that the degree of the stenosis measured by this single indicator was representative for the stenosis condition during the whole hospital stay.

Six severity categories hased on the degree of coronary stenosis were distinguished (table 6.2.). In total 39 patients had ' $n$ ' $^{\prime}(n=23)$ or 'decreasing to no significant coronary stenosis' $(n=16)$. The majority of the patients had significant sienosis varying from low degree of stenosis $(n=20)$ to moderate stenosis $(n=37)$ and highly severe stenosis $(n=57)$. For 29 patients the degree of stenosis did increase during hospital stay. 
Table 6.2 Severity categories describing the (change in) degree of coronary stenosis.

Severity category

0
1
2
3
4
5

Subtotal of relevant cases

Missing cases

Total
Description

Frequencies

no sig. stenosis

23

decreasing to no sig. stenosis

16

(consistent) low degree of stenosis

20

(consistent) moderate degree of stenosis

increasing stenosis

(consistent) high degree of stenosis

The extent of coronary stenosis.

Extent measures the spread of stenosis within the coronary artery system. The exient of stenosis comprises the number of coronary arteries with any kind of stenosis, regardiess the degree and the specific location. If no significant stenosis $(<70 \%)$ is present in any of the three arteries, patients received severity weight ' 0 '. If a significant stenosis is present in only one artery patients get severity weight ' 1 '. If there were two arteries present with a significant stenosis patients get severity weight ' 2 '. The highest severity weight of ' 3 ' is given to patients who had a significant stenosis in all three primary arteries.

\section{Severity Categories.}

The same rules are applied that are used for the severity trends concerning the intensity of stenosis. The extent is distinguished into 5 severity categories (table 6.3.). Most patients $(n=82)$ had significant stenosis in only one artery, in 42 patients stenosis was present in two arteries and in a small group of 20 patients three arteries showed stenosis.

Table 6.3 Severity categories describing the extent of coronary stenosis.

Severity category

Description

Frequencies

0

I

no sig. stenosis

2

decreasing to no sig. stenosis

Subtotal of relevant cases

Missing cases 
The location of coronary stenosis.

It is generally assumed that the location of stenosis (or occlusion) in a coronary artery is important, when one is interested in measuring the severity of this deficiency. It stands to reason that a stenosis in the beginning (proximal segment) of an artery will reduce or obstruct the blood supply over a larger area than when a stenosis occurs at the end (distal segment) of an artery. Therefore each arterial segment is given a severity weight. The severity weights were determined with the help of the panel of cardiologists, who used mortality risk as one of the principal criteria. The severity weights for the location of stenosis are given in table 6.4 .

Table 6.4 Severity weights for the location of coronary stenosis.

\begin{tabular}{|c|c|c|c|c|c|c|c|c|c|}
\hline $\begin{array}{l}\text { primary } \\
\text { comonary } \\
\text { artery }\end{array}$ & prox & marg & mid & dist & $\begin{array}{c}\text { mid+ } \\
\text { dist }\end{array}$ & $\begin{array}{l}\text { post } \\
\text { desc }\end{array}$ & $\begin{array}{c}\text { post } \\
\text { lat }\end{array}$ & $\begin{array}{l}\text { first } \\
\text { diag }\end{array}$ & $\begin{array}{l}\mathrm{sec} . \\
\text { diag }\end{array}$ \\
\hline$R C A$ & 5 & - & 4 & 3 & - & 3 & & & \\
\hline$L A D$ & 8 & - & 5 & 2 & - & - & - & 2 & 2 \\
\hline$R C X$ & 6 & 1.5 & - & - & 4.5 & 3 & 1.5 & - & - \\
\hline
\end{tabular}

First, the severity for each coronary artery is calculated by taking the highest severity weight occurring within the artery. It is expected that the highest severity weight within the segments of an artery is sufficient to indicate the severity of the stenosis of that artery. Next, the location based severity score with respect to the stenosis concerning the overall coronary artery system is obtained by taking the sum of the individual segments scores. Theoretically this total score may vary between 1.5 - 19 for each patient. The total score for a patient will give the overall severity based on the location of the coronary artery stenosis.

Severity Categories.

The scores of the lecation of coronary stenosis were recoded into six categories (table 6.5.). The largest category of patients has stenosis in minor $(n=50)$ or moderately severe locations $(n=43)$. About 30 patients show significant stenosis in severe locations and 2 I patients have stenosis in highly severe locations.

Table 6.5 Severity categories describing the lecation of coronary stenosis.

\begin{tabular}{cclc}
\hline Severity category & Score & Description & Frequencies \\
0 & 0 & no sig. stenosis & 23 \\
1 & $x-0$ & decreasing to no sig. stenosis & 15 \\
2 & $1.5-5.0$ & stenosis in minor locations & 50 \\
3 & $5.5-9.5$ & stenosis in moderately severe locations & 43 \\
4 & $10.0-13.0$ & stenosis in severe locations & 30 \\
5 & $13.5-19.0$ & stenosis in highly severe locations & 21 \\
Subtotal of relevant cases & & 182 \\
Missing cases & & & 282 \\
Total & & 46.4 \\
\hline
\end{tabular}




\subsubsection{Severity of myocardial ischemia.}

The etiology underlying myocardial ischemia can be regarded as the discrepancy between the myocardial need for oxygen and the amount of oxygen in the blood delivered to the myocardium via the coronary arteries. Different indicators to measure the severity of myocardial ischemia are presented in the model below.

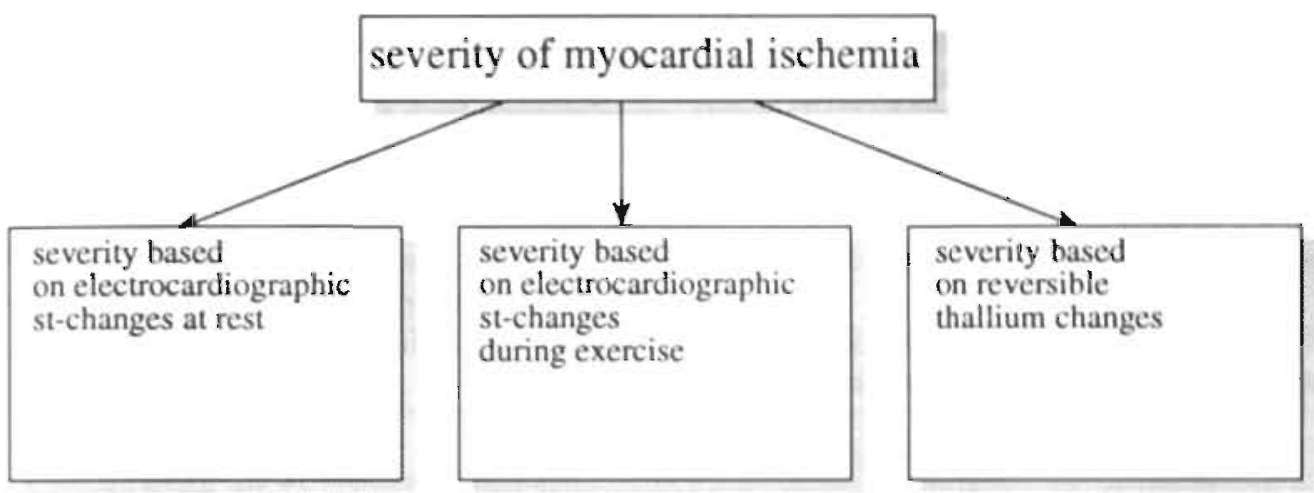

\subsubsection{Severity based on electrocardiographic ST-changes at rest.}

When myocardial ischemia is present this can be detected by elevation or depression of the ST-segment (henceforth ST-change) in several leads of the ECG. ST-changes in distinct leads are an indication for the location and the extent of the ischemic segments of the myocardium. ST-changes are recorded separately for the following left ventricle wall segments: anterior, septal, lateral, posterior and inferior ${ }^{4}$. The wall segments scores are distinguished into: (0) no ST-segment changes and (1) significant ST-segment changes (a ST elevation or depression with a sum of at least $3 \mathrm{~mm}$.) indicating myocardial ischemia. Because of this rather crude dichotomy the intensity of the ischemia in the left ventricle cannot be calculated.

The extent of myocardial ischemia.

The number of ischemic wall segments according to ST-changes are used to construct a severity index. When ischemic wali segments or no ST-changes are present patients are given severity weight ' 0 '. If ST-changes conrespond with only one wall segment or two adjacent wall segments, it is likely that myocardial ischemia is occuring in a rather small area, so a severity weight of ' $\mathrm{l}$ ' is given. If patients have three wall segments according to significant ST-changes, they get severity weight ' 2 '. The highest severity weight of ' 3 ' is given to patients with ST-changes corresponding with four or five ischemic left ventricular wall segments.

4 significant ST-segment changes are defined as ST elevation or depression with a sum, of at least $3 \mathrm{~mm}$.:

Septal : $\quad$ ST-changes in leads V1-V2

Anterior: ST-changes in leads V2-VS

Lateral : $\quad$ ST-changes in leads I, AVL, V6

Posterior: $\quad$ ST-changes in leads V1, V2 and T-wave changes in leads VI, V2

Inferior: ST-changes in leads II, III, A.VF. 


\section{Severity Categories.}

Ten different severity patterns could be discerned (table 6.6.; a to j). Only a few patients had no ST-changes at all, or their ST-changes on admission (T0) disappeared subsequently $(\mathrm{n}=$ $25)$; these severity patterns are combined in one category with weight. ' 0 '. A large group of patients ( $\mathrm{n}=187$ ) had consistent ST-changes corresponding with one or two wall segments; they form one category with weight ' 1 '. Patients with consistent ST-changes corresponding with three or more wall segments $(n=210)$ were put together in the category with weight ' 2 '. The three different patterns of increasing ST-changes concerning 40 patients are combined into the category with weight ' 3 '. This category also includes a small heterogeneous group of five patients with increasing as well as decreasing ST-changes. Eventually four different severity categories can be distinguished on the basis of the extent of ST-changes measured by the ECG at rest.

Tuble 6.6 (Combined) Severity patterns and categories describing the extent of ST-changes during the total hospital stay measured by ECG at rest.

\begin{tabular}{|c|c|c|c|c|}
\hline $\begin{array}{l}\text { Severity } \\
\text { pattern }\end{array}$ & Description & Freq. & $\begin{array}{l}\text { Severity } \\
\text { category }\end{array}$ & $\begin{array}{r}\text { Combined } \\
\text { freq. }\end{array}$ \\
\hline$a$ & no sig. ST.changes in TO-T3 & 9 & & \\
\hline$b$ & $\begin{array}{l}\text { disappearance of ST-changes in I or } 2 \\
\text { wall segments }\end{array}$ & 8 & 0 & 25 \\
\hline$c$ & $\begin{array}{l}\text { disappearance of ST-changes in } 3 \text { wall } \\
\text { segments }\end{array}$ & 8 & & \\
\hline$d$ & $\begin{array}{l}\text { consistent ST-changes in } 1 \text { or } 2 \text { wall seg- } \\
\text { ments }\end{array}$ & 187 & 1 & 187 \\
\hline$e^{\prime}$ & consistent ST-changes in 3 wall segments & 178 & & \\
\hline$f$ & $\begin{array}{l}\text { consistent ST-changes in } 4 \text { or } 5 \text { wall seg- } \\
\text { ments }\end{array}$ & 32 & 2 & 210 \\
\hline$g$ & $\begin{array}{l}\text { increasing } S T \text {-changes from } 0 \text { in } T 0 \text { to } 1 \\
\text { or } 2 \text { wall segments in } T 1-T 3\end{array}$ & 17 & & \\
\hline$h$ & $\begin{array}{l}\text { increasing } S T \text {-changes from } 1 \text { or } 2 \text { in } T 0 \\
\text { to } \geq 3 \text { wall segments in } T I-T 3\end{array}$ & 13 & & \\
\hline i & $\begin{array}{l}\text { increasing } S T \text {-changes from } 1 \text { or } 2 \text { in } T 0 \\
\text { or } T I \text { to } \geq 3 \text { wall segments in } T 2-T 3\end{array}$ & 5 & 3 & 40 \\
\hline$j$ & $\begin{array}{l}\text { increasing ST-changes in } T 1 \text { and decre- } \\
\text { asing } S T \text {-changes in } T 2 \cdot T 3\end{array}$ & 5 & נ] & \\
\hline Subtotal & & 462 & & 462 \\
\hline Missing cases & & 2 & & 2 \\
\hline Total & & 464 & & 464 \\
\hline
\end{tabular}

The location of myocardial ischemia.

A distinction must be made between ST-changes in the anteroseptal wall segments and those in other wall segments in the left ventricle. ST-changes in the anteroseptal wall segments are. assumed to be more severe. Therefore patients with ST-changes in leads V1 - V2 will get a severity weight ' 2 ' in comparison to patients with ST-changes in other leads, who get ' 1 '. If no ST-changes were present patients receive a severity weight of ' 0 '. 


\section{Severity Categories.}

There appear to be seven different patterns in the location of ST-changes during hospital stay (table 6.7.; a to g). Patients with no ST-changes at all or disappearing ST-changes during hospital stay $(n=23)$ are combined in one category with weight ' 0 '. Patients with consistent or increasing ST-changes in non-anteroseptal lead's $(n=280)$ are put together in the category with weight ' 1 '. Patients with consistent anteroseptal ST-changes $(n=150)$ all remain in one category' with weight ' 2 '. Patients with increasing ST-changes from non-anteroseptal to anteroseptal during hospital stay $(n=9)$ were put into a higher severity category (3). Eventually four different severity categories are distinguished based on the location of STchanges measured by the ECG at rest.

Table 6.7 (Combined) Severity patterns and categories describing the location of ST-changes during the total hospital stay measured by ECG at rest.

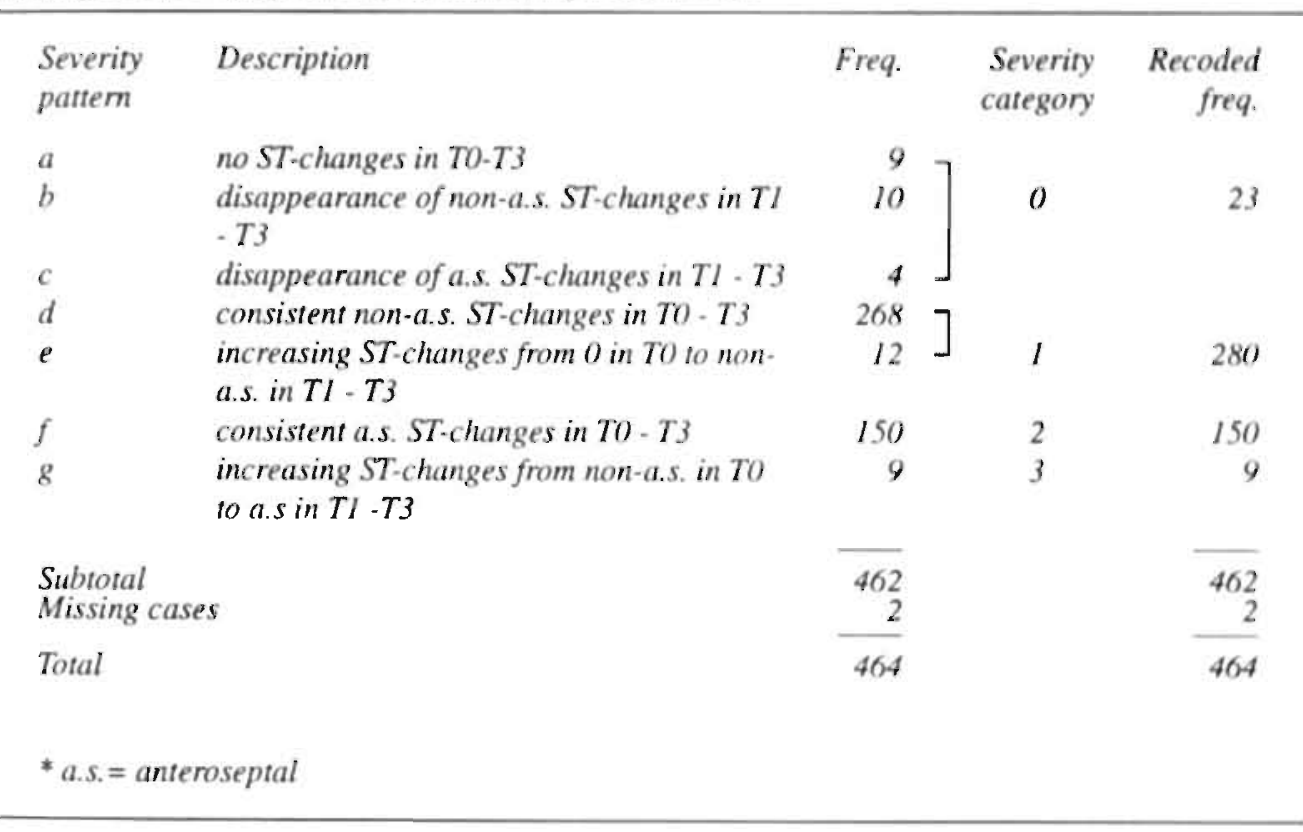

\subsubsection{Severity based on electrocardiographic ST-changes during exercise.}

Myocardial ischemia can also be detected by ST-changes during exercise. The exercise test (according to the so-called Bruce-protocol) is designed to gauge the functional capacity of the heart. The basis of this test is the increased myocardial oxygen consumption caused by exercise. The Bruce test is performed normally just before a patient's discharge from hospital. During the course of a progressive increase in exercise load the patient's electrocardiogram is monitored for evidence of additional myocardial ischemia.

Results of the Bruce test are measured by (a) typical ST-changes in the ECG (b) the level of exercise load and (c) the absence/presence of ischemic pain during the test. Results of the ECG-findings are recorded into: (0) no typical ST-changes and (1) presence of typical ST changes. Also the highest achieved level of exercise load is recorded (ranging from I "low effor' to 5 'very high effort'). 
To measure severity of myocardial ischemia during exercise even four dimensions are assumed to be important. The scale representing these four dimensions are the intensity, extent and location of myocardial ischemia plus the degree of additional ischemia compared to the necrotic zone.

The intensity of myocardial ischemia.

This dimension refers to the presence or absence of ST-changes in and outside the necrotic zone and to the highest exercise load reached by a patient while being tested. The STchanges are measured at the maximum degree of exercise load when ST-changes occur. It is to be expected that ST-changes occurring during a low exercise load are more severe then during a high exercise load. First a distinction was made into patients with no ST-changes during any kind of exercise load (severity weight ' $0^{\prime}$ ) and patients with ST-changes. The latter were further distinguished into severity groups according to their maximal level of exercise load reached during the Bruce test. Only few patients endured an exercise load of stage 4 and 5 ; since these form adjacent categories they are given the same severity weight of '1'. Patients with ST-changes occurring during an exercise load at stage 3 and stage 4 are respectively given severity weights ' 2 ' and ' 3 '. The highest severity weight is given to patients with ST-changes enduring a minimum of exercise; they were given severity weight ' 4 '. The number of patients in each severity category are presented in table 6.8.

Table 6.8 Severity categories describing the intensity of ST-changes during exercise.

Severity category

0

1

2

?

4

Subtotal of relevant cases

Missing cases

Total
Description

Frequencies

$\begin{array}{ll}\text { ST-changes at stage } 2 & 53 \\ \text { ST-changes at stage I } & 25\end{array}$

\section{The extent of myocardial ischemia.}

If is assumed that the severity of ischemia during exercise increases when patients have. ECG-leads which show ST-changes. The number of ischemic wall segments according to ST-changes is used to develop a severity scale. When no ischemic wall segments are piesent the severity weight ' 0 ' is given to the patients. If patients have one or two ischemic wall segments, severity is assumed to be about equal and they are given severity weight ' 1 '. When three ischemic wall segments are suspected according to the ST-changes the given severity weight is ' 2 ' and patients with four ischemic wall segments are categorized into severity group ' 4 '. There are no patients with ST-changes in all five wall segments in our population. The number of patients in each severity category is presented in table 6.9 . 
Table 6.9 Severity categories describing the extent of ST-changes during exercise.

Severity category

0

I

2

3

Subtotal of relevant cases

Missing cases

Total
Description

no ST-changes

ST-changes in 1 or 2 wall segments

$S T$-changes in 3 wall segments

$S T$-changes in 4 wall segments
Frequencies

$\begin{array}{r}70 \\ 105 \\ 21 \\ 7 \\ \hline 203 \\ 261 \\ \hline 465\end{array}$

The location of myocardial ischemia.

If ST-changes corresponding with the anteroseptal wall segments of the left ventricie are present it is assumed that the condition of those patients is more severe. Patients with 'anteroseptal' ST-changes are given severity weight ' 2 ', in contrast to other patients which are given severity weight ' 1 '. If no ST-changes were present the severity weight is ' 0 '. The number of patients in each category is presented in table 6.10.

Table 6.10 Severity categories describing the location of ST-changes during exercise.

Severity category

0

I

2

Subtotal of relevant cases

Missing cases

Total
Description

no ST-changes

non-anteroseptal ST-changes

anteroseptal ST-changes
Frequencies

70

100

33

203

261

464

The degree of additional ischemia.

One of the specific purposes of applying a Bruce test is to detect the presence of additional ischemia, on top of the ischemia at the necrotic zone. To measure the severity of additional ischemia, results from the ECG at rest are used together with results from the ECG during exercise. The necrotic zone is identified using typical Q-waves in the ECG at rest (see \& 6.2.3.). A distinction is made between four severity categories with different severity weights for additional ischemia. Patients with no ST-changes are again given severity weight ${ }^{\prime} 0$ '. If ST-changes appear only in the leads with $Q$-waves, a severity weight of ' 1 ' is given. If patients had ST-changes in the leads with Q-waves plus in the leads with no Q-waves or if ST-changes exclusively occur in the leads with no Q-waves, additional ischemia is suspected, and the patient is given severity' weight ' 2 '. The majority of patients who had no. Q-waves at all during hospital stay $(n=56)$ had no exercise ECG and were classified as missing cases $(n=35)$. Of the remaining patients who had no Q-waves at all, 11 patients had no ST-changes during exercise and in 10 patients ST-changes were present; they are classified in severity category ' 2 '. The number of patients in each category is presented in table 6.11 , 
Table 6.11 Severity categories describing additional ischemia during exercise compared to myocardial necrosis.

Severity category

0

I

2

Subtotal of relevant cases

Missing cases

Description

no ST-changes

ST-changes on same location

ST-changes on same and additional location

or ST-changes only on additional location
Frequencies

70

46

87

Total

\subsubsection{Severity based on 'reversible thallium changes'.}

Thallium outlines cardiac locations with reversible and non-reversible myocardial ischemia. Particularly during exercise, the nuclides 'decrease' in ischemic zones and 'increase' in nonischemic zones. These ischemic zones are visualized by a reduced nuclear activity during exercise followed by a normal nuclear activity after exercise (so-called redistribution). The thallium test is performed normally just before a patient's discharge from hospital.

According to the thallium test the left ventricular wall can be subdivided into seven wall segments: septal, anterior, apical, lateral, posterior, inferior and basal. For each wall segment. the scores (0) which means no thallium changes present at all, or (1) which stands for (partially) redistributed thallium changes are recorded.

From a clinical point of view a distinction has to be made between partial redistribution and complete redistribution. 'Complete redistribution' means that the cardiac area was encounterd by acute reversible myocardial ischemia; partial redistribution means that the cardiac area may he injured by 'subacute' reversible myocardial ischemia or myocardial necrosis. However, only few patients in our population showed partially redistributed thillium.

The extent of myocardial ischemic.

It is assumed that the severity of myocardial ischemia increases, if more wall segments show typical thallium changes. The extent of reversible thallium changes is measured by the number of wall segments with thallium changes. If no thallium changes occurred then patients are given severity weight ${ }^{\circ} 0^{\circ}$. Patients with thallium changes in one or two wall segments are considered to be about equal as far as severity is concerned and are given severity weight ' 1 .". Patients with thallium changes in more than two wall segments did not occur in our data. The number of patients in each category is presented in table 6.12 . 
Table 6.12 Severity categories describing the extent of reversible thallium changes.

Severity category

0

I

Subtotal of relevant cases

Missing cases

Total
Description

no reversible thallium changes

reversible thallium changes in I or 2 wall segments
Frequencies

67

19

86

378

464

The location of myocardial ischemia.

As was mentioned before it is assumed that myocardial ischemia in the anteroseptal wall of the left ventricle has a more severe prognosis than in other wall segments. If no reversible thallium changes are present, patients are allocated to the lowest category with severity weight ' 0 '. When reversible thallium changes are present in anterior, septal or apical wall segments, patients get the highest severity weight of ' 2 ', in comparison 10 patients with thallium changes in the other four wall segments who receive severity weight ' 1 '. The number of patients in each category is presented in table 6.13.

Table 6.13 Severity categories describing the location of reversible thallium changes.

Severity category

o

I

2

Subtotal of relevant cases

Missing cases

Total
Description

no reversible shallium changes

reversible thallium changes non-anterosep-

ral

reversible thallium changes anteroseptal

Frequencies

67

16

3

86

378

464

\subsubsection{Severity of myocardial necrosis.}

Myocardial necrosis is generally caused by thrombotic occlusion of coronary arteries. The location and the extent of the infarction will depend on the anatomical structure of the arteries, the site of current and previous occlusions and the adequacy of the collateral circulation. Acute myocardial necrosis is characterized by irreversible injury of the heart muscle. The severity of these irreversible changes is very important with respect to the remaining ventricular function. Several techniques can be used to measure the heart's irreversible damage. Among the techniques of observation used by cardiologists, results from electrocardiograms, laboratory tests and thallium tests are: supposed to be primary guidelines in specifying and detecting the severity of myocardial necrosis. 


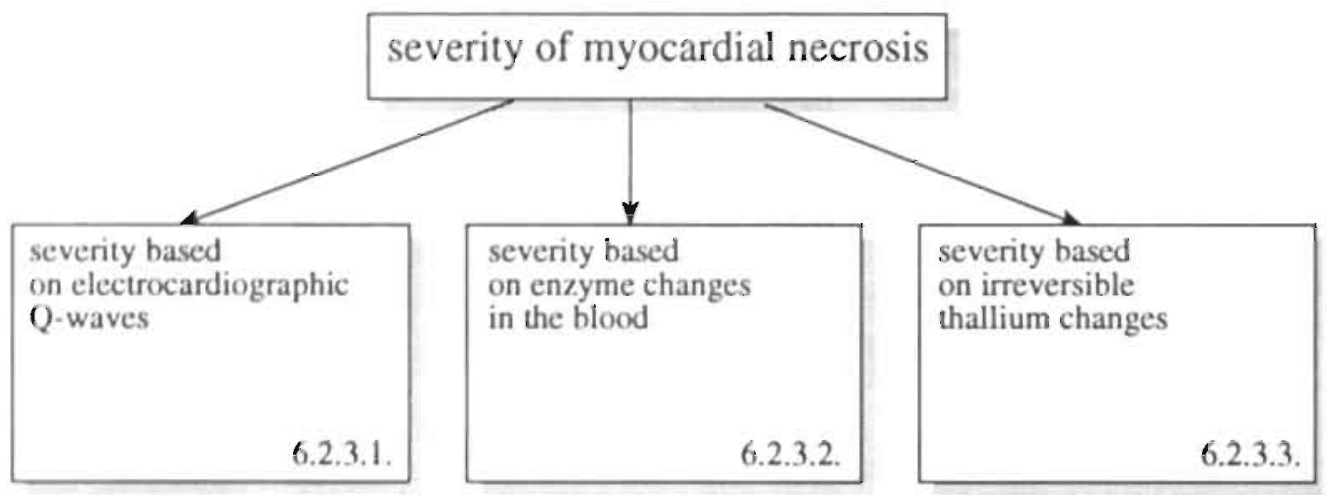

\subsubsection{Severity based on electrocardiographic Q-waves.}

The electrocardiogram (ECG) is a graphic record of the electrical currents generated by the heart. The ECG can show which portion of the heart has probably been damaged. It measures to a certain extent the magnitude of the damage. Specific ECG characteristics for myocardial necrosis are most frequently the disappearance of the so-called R-wave and the formation of the so-called Q-wave. These Q-waves are not always present at the moment of admission but can develop within several hours. Q-waves on the ECG give an indication of presence as well as location of myocardial necrosis.

Characteristic Q-waves on the ECG are collected for each lime period. If more than one ECG was performed in one period, the one most. deviant from the normal situation is considered to be relevant. The necrotic wall segments of the left ventricle, observed by the ECG, can be distinguished into: septal, anterior, lateral, posterior and inferiors.

The results of the ECG interpretation were used to distinguish the characteristic Q-waves in each group of leads, corresponding with each wall segment into: 0: no characteristic Qwaves and 1: Q-waves indicating necrosis.

\section{The extent of myocardial necrosis.}

Necrosis extending over more than two wall segments is assumed to be more severe than necrosis restricted to one or two wall segments. Although the extent of necrosis is difficult to measure using ECG, its data are often used to predict to some degree the magnitude of necrosis. In this study extent refers to the number of wall segments with typical Q-waves. It is assumed that severity of myocardial necrosis will increase when $Q$-waves are found in the ECG leads representing more wall segments.

If no Q-waves are present patients get a severity weight of ' 0 '. Patients with $\mathrm{Q}$-waves corresponding with one or two wall segments are considered to be equivalent as far as severity is concerned: they are given a weight of ' 1 '. If there are three or four (or five) wall segments with Q-waves, a severity weight of respectively ' 2 ' and ' 3 ' is given.

5 Typical Q-waves are defined:

Septal Q-waves in leads V1-V2 (V3)

Anterior: Q-waves in leads V2-VS

Lateral: Q-waves in leads 1, AVI., Vo

Posterior: Inversed $\mathrm{Q}$ or high $\mathrm{R}$-waves in leads VI, V2 and T-wave changes in V!, V2

Inferior: Q-waves in leads II, III, AVF. 


\section{Severity Categories.}

As far as the extent of necrosis determined by significant Q-waves in the left ventricle is concerned, there appear to be four different severity categories in the patient population at hand (see table 6.14.). Patients with no significant Q-waves present during the total hospital stay are grouped into one category with severity weight $0^{\prime}$ ': the number of patients in this category is 56. Most patients $(\mathrm{n}=331$ ) have consistent $\mathrm{Q}$-waves corresponding with 1 or 2 wall segments with no changes during hospital stay; they form the second category with severity weight ' 1 '. Patients with consistent Q-waves corresponding with three wall segments $(n=46)$ and patients with increasing $Q$-waves in wall segments $(n=29)$ are grouped into one category with severity weight ' 2 '. Q-waves corresponding with four or five wall segments do not occur in the population. In total three severity categories representing myocardial necrosis are distinguished based on the extent of significant Q-waves measured by the ECG at rest.

Table 6.14 (Combined) Severity patterns and categories representing the extent of Q-waves during the total hospital stay measured by ECG at rest.

\begin{tabular}{|c|c|c|c|c|}
\hline $\begin{array}{l}\text { Severity } \\
\text { pattern. }\end{array}$ & Description & Freq. & $\begin{array}{l}\text { Severity } \\
\text { Caregory }\end{array}$ & $\begin{array}{r}\text { Combined } \\
\text { freq }\end{array}$ \\
\hline$a$ & no $Q$-waves in $T O-T 3$ & 56 & 0 & 56 \\
\hline$b$ & $\begin{array}{l}\text { consistent } Q \text {-waves in } 1 \text { or } 2 \\
\text { wall segments in TO-T3 }\end{array}$ & 331 & $I$ & 311 \\
\hline$c$ & $\begin{array}{l}\text { consistent } Q \text {-waves in } 3 \text { wall } \\
\text { segments in } T O-T 3\end{array}$ & 46 & & \\
\hline$d$ & $\begin{array}{l}\text { inaeasing } Q \text {-waves from } O \text { in } \\
T O 101,2 \text { or } 3 \text { wall segments in } \\
T I-T 3\end{array}$ & 29 & 2 & 75 \\
\hline \multicolumn{2}{|c|}{ Subtotal of relevant cases } & 462 & & 462 \\
\hline \multicolumn{2}{|c|}{ Missing cases } & 2 & & 2 \\
\hline Total & & 464 & & 464 \\
\hline
\end{tabular}

The location of myocardial necrosis.

A separate severity indicator is constructed which refers to the location of necrosis in the myocardium. It is assumed that Q-waves corresponding with the anteroseptal site will be more severe than in the other sites of the left ventricle. If no Q-waves are present, these patients are given severity weight ' 0 '. In case Q-waves are present corresponding with nonanteroseptal wall segments, patients receive severity weight ' 1 '. Patients with Q-waves corresponding with the anteroseptal wall segment are given the highest severity weight of ' 2 '.

\section{Severity Categories.}

The severity of myocardial necrosis measured by the location of Q-waves during hospital. stay can be represented by three different categories (table 6.15.). Once again patients with 
no significant Q-waves during stay $(\mathrm{n}=56)$ form one category and are given severity weight ' 0 '. Patients with consistent non-anteroseptal Q-waves $(n=275)$ form a second category with severity weight ' 1 '. Patients with consistent anteroseptal $Q$-waves $(n=122)$ and patients with a deterioration from non-anteroseptal to anteroseptal Q-waves in a later phase of the stay $(n=9)$ are brought together in one category with severity weight ' 2 '.

Tuble 6.15 (Combined) Severity patterns and categories representing the location of Q-waves during the total hospital stay measured by ECG at rest.

\begin{tabular}{|c|c|c|c|c|}
\hline $\begin{array}{l}\text { Severity } \\
\text { pattem }\end{array}$ & Description & Freq. & $\begin{array}{l}\text { Severity } \\
\text { category }\end{array}$ & $\begin{array}{r}\text { Combined } \\
\text { freq. }\end{array}$ \\
\hline$a$ & no Q.waves $T(0 \cdot T 3$ & 56 & 0 & 56 \\
\hline$b$ & consistent non a.s. ${ }^{*} Q$-waves in TO- $T 3$ & 275 & l & 275 \\
\hline 6 & consistent a.s. Q-waves in TO-T3 & 122 & & \\
\hline$d$ & $\begin{array}{l}\text { increasing } Q \text {-waves from } 0 \text { or non } \\
\text { a.s. in To to a.s. } Q \text {-waves in } T 1-T 3\end{array}$ & 9 & 2 & I3I \\
\hline \multicolumn{2}{|c|}{ Subrotal of relevant cases } & 462 & & 462 \\
\hline \multicolumn{2}{|c|}{ Missing cases } & 2 & & 2 \\
\hline Toral & & 464 & & 464 \\
\hline
\end{tabular}

\subsubsection{Severity of myocardial necrosis measured by enzyme changes in the blood.}

When hear muscular cells die, typical cellular enzymes are secreted into the blood. Within the lirst days of a myocardial necrosis there will be an abnormal rise of these enzymes in the blood. After several days the enzyme release will return to its normal level. The enzyme release can be detected through laboratory analysis of the blood. The enzymes most commonly analyzed are Serum Glutamic Oxalacetic Transaminase (SGOT), Lactic Dehydrogenase (LDH) and Creatine Phosphokinase (CPK). The enzyme release during the course of the infaretion can often be used to estimate the size of the infarction. However, not only the value of enzymes but also the time-span in hours in which these enzymes "peak" is an important indicator of the severity of myocardia! necrosis.

For all patients the following data have been recorded for each time period of their stay:

- hours of pain

: the duration of ischemic pain from onset of pain to the hospital admission:

- SGOT-value : the highest SGOT-value measured in a time period;

- SGOT-peak value time : the time in hours after which the SGOT-peak is reached,

- CPK-value measured from the moment of hospital admission;

- CPK-peak value time : the time in hours after which the CPK-peak is reached.

- LDH-value measured from the moment of hospital admission;

the highest LDH-value measured in a time period; the time in hours to reach the LDH peak is considered to be of less importance as far as severity of necrosis is concerned. 
The mean duration of ischemic pain from onset of pain to the admission at the hospital is approximately four hours, but looking at the median, most patients have only one or two hours of pain. The mean time after which an enzyme peak is reached in hospital, is 20 hours for SGOT and 18 hours for CPK.

\section{Types of patients.}

Since the enzyme release pattern can differ for certain types of patients, for each type a different approach to data analysis must be taken. Our research population can be distinguished into:

1. patients with acute myocardial necrosis whose enzyme peak is reached during the acute phase (T-0);

2. patients with acute myocardial necrosis whose enzyme peak is reached within three days (T-1); this is the common pattern (see figure 6.16);

Figure 6.16. Enzyme release pattern in patients with acute myocardial necrosis measured by a peak in $\mathrm{T}-1$.

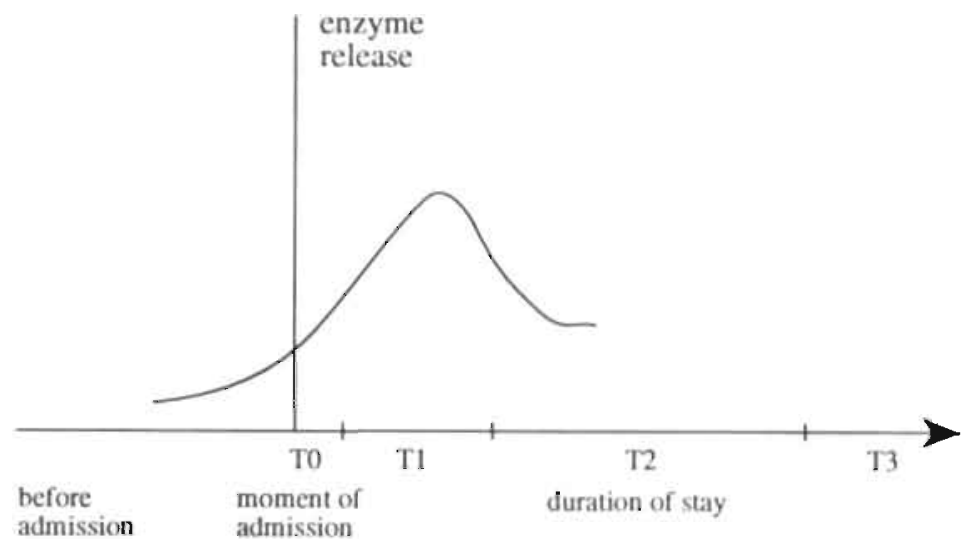

3. patients with acute myocardial necrosis whose enzyme peak is reached after three days (T-2);

4. patients with recurrent myocardial necrosis show in general two enzyme peaks, one in the first part of the stay and another caused by a second increased enzyme release (see figure 6.17); 
Figure 6.17. Enzyme release pattern in patients with recurrent myocardial necrosis measured by peaks in T-1 and T-2.

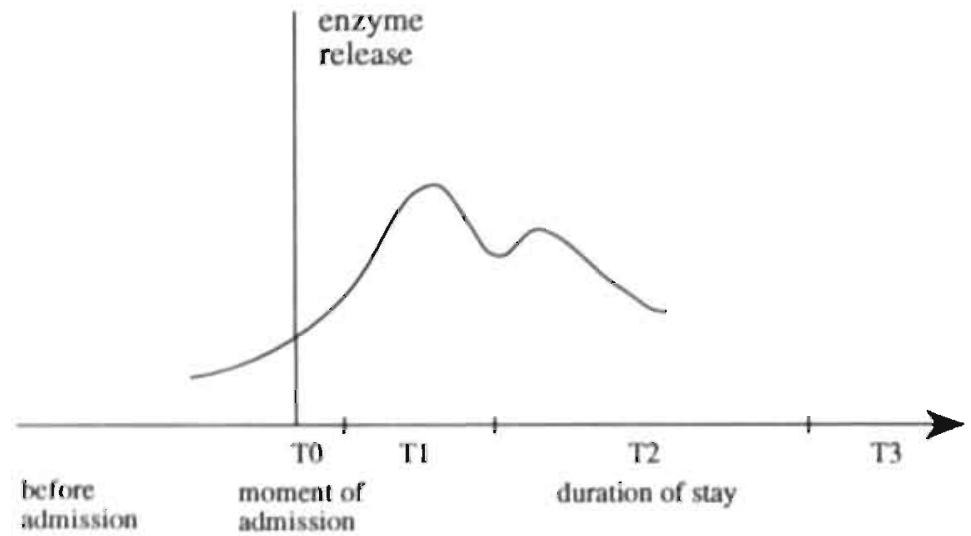

5. patients, with subacute myocardial necrosis (necrosis happening jusi before admission to the hospital) who have an enzyme peak just before hospital admission (see figure 6.18).

Figure 6.18. Enzyme release pattern in patients with subacute myocardial necrosis measured by a peak before admission.

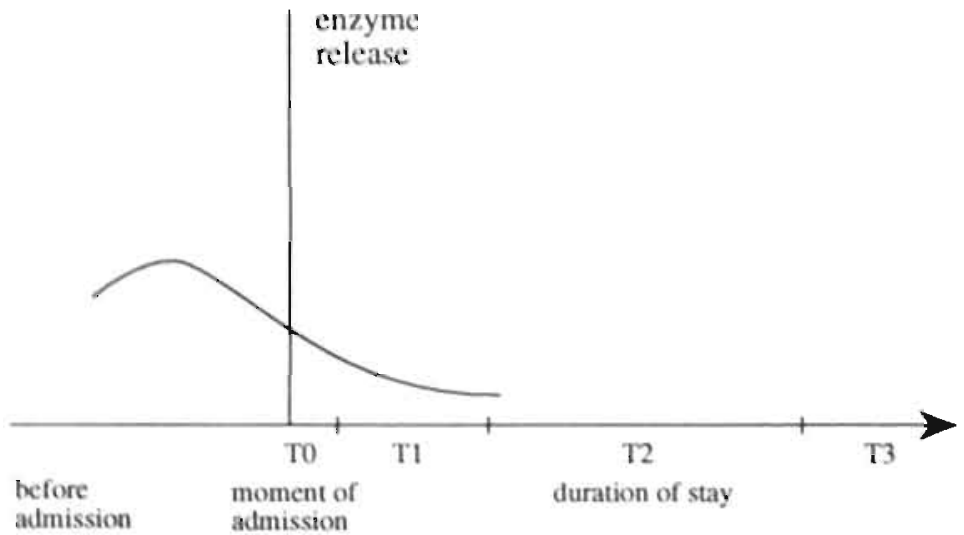

Measurement of severity of myocardial necrosis.

A generally accepted rule in cardiology (De Zwaan e.a., 1988) is that the highest enzyme peak value during stay times the time-span in hours to reach this peak will give an indication of the total amount of enzymes released during the phase of development of necrosis. If the total amount of enzymes increases, the infaret-size and severity of necrosis is assumed to increase. So, to measure the severity of necrosis based, on enzymatic changes, the following 
general rule is applied; an area measure is calculated multiplying the highest enzyme value during stay with the time in hours after which the peak was reached from the very first moment the patient is complaining of typical ischemic pain.

Because of the different types of enzyme curves, it will be necessary to adapt this general rule to more specific situations. A further description of constructing the SGOT-area index is given below.

If the enzyme peak falls in time periods $\mathrm{T}-0$ or $\mathrm{T}-1$ the following formula is applied:

SGOT area measure $=$ the highest SGOT-value during hospital stay (SGOTV) * (pain before admission in hours (PBA) + the time in hours until the SGOT-peak is reached starting from the moment of admission (SGOTT)).

If the enzyme peak falls in time period T-2 or T-3, the formula was shortened to: SGOT= SGOTV * SGOTT. It is assumed that if the enzyme peak occurs after 72 hours, the duration of pain before admission (PBA) is cvidently irrelevant.

If patients have enzyme values below or equal to their so-called normal value, they are expected to have no necrotic abnormalities. Therefore, all enzyme values have to be corrected by their normal values. This implies that in both formulas described the SGOTV has to be replaced by: (SGOTV minus 'normal' value). If the enzyme scores are below this standard, the calculated arca measure is set to zero: no significant enzyme release. If a negative area measure is obtained, the patients concerned also receive a zero value. Mean 'normal' enzyme values, that are used in our analysis are: SGOT: $20 \mathrm{U} / \mathrm{l}, \mathrm{CPK}: 90 \mathrm{U} / \mathrm{h}$ and LDH :250 Un.

If a patient is also suffering from liver disorders certain types of enzymes are expected to be unspecific to predict the severity of myocardial necrosis. The enzymes SGOT and LDI, are particularly influenced by liver disorders. The presence and amount of cell destruction in the liver is specifically measured by the enzyme called Serum Glutamic Pyruvatic Transaminase (SGPT). If the SGPT-score is higher than $100 \mathrm{U} /$, the SGOT and LDH scores are considered to be unreliable: consequently eacht type of enzyme measure is considered as missing for these patients.

The same formula is applied if patients have recurrent necrosis during their hospital stay $(\mathrm{n}=11)$. The duration of the second enzyme peak then starts from the moment that the patient experiences heavy ischemic pain for the second time during hospital stay and ends when a second enzyme peak is reached. Two area measures could be calculated but the latter is used as the most relevant.

Missing data.

In applying the ruies above, we did encounter considerable problems with regard to missing data. The duration of ischemic pain before admission to the hospital was unknown (i.e. lacking in the medical record) for 121 of the 464 patients $(26 \%)$. Another problem concerns patients with so-called subacute infarction whose cmyme peaks are unavoidably underestimated because they had their enzyme peak before hospital actmission. If one of more scores in the formulas were missing, the patients concerned are generally excluded from further data analysis. To minimize this loss of cases, theoretical values have been substituted for missing data. In three specific instances substitution of theoretical values for missing data has taken place: 
- Patients with unknown duration of ischemic pain before hospital admission, while their peak value time was known. In this situation the mean duration in which an enzyme peak is normally reached ( 24 hours in case of SGOT and 20 hours in case of (PK) has been substituted for the total peak value time (including duration of ischemic pain).

- Patients, with an unknown peak value time, while the duration of ischemic pain before hospital admission was known. For a large part this concemed patients with a subacute infarction, who on admission already had the highest enzyme score. In case an enzyme peak is reached in period T-0, a period of 4 bours is substituted for the unknown peak value time, because it is expected that the first laboratory results would be available by that time. In some cases the duration till the enzyme peak is reached in T-1 was unknown; the total duration is then substituted using the same rule as described for the situation above.

- Patients with both unknown duration of ischemic pain and unknown peak value time. If boch variables were unknown and the enzyme peak occurs in T-0 or T-1, the same rule as described above is applied for the total time period concerned.

Substitution of theoretical values for missing data could imply that the reliability of the severity-indices is affected, certainly in the cases of patients with a sub-acute infarction. On the other hand, the number of patients included in analysis is salvaged as much as possible and the severity-indices may have become more representative. In order to study the effecl of the substitution, the severity indices in both instances (missing data excluded and included) have heen calculated. Correlations between the three enzyme severity-indicators did increase somewhat (especially in T-0) due to the inclusion of patients with substituted data. Comparing the oufcome of both versions it was concluded that the inclusion of the theoretic values does no substantially harm to the original means and standard deviations. Thus, more patients could be included in subsequent analyses. The enzyme release with inclusion of theoretical values will henceforth be used.

\section{Severity Categories.}

The enzyme release is a reliable and often used measure for the patient's severity of myocardial necrosis with respect to his or her hospital stay. Therefore, this measure is taken as an indicator for the severity of myocardial necrosis. If the enzyme peak falls in period TO then the area measure of $\mathrm{TO}$ is taken, if the enzyme peak falls in Tl the area measure from Tl is taken and so on. An exeeption is made for patients with a recurrent infarction while in hospital. For these patients $(n=12)$ the enzyme output can rise again and a change in severity of necrosis measured by enzyme values will then be relevant. For these patients the enzyme release of the first infaretion is taken and the second infaretion is seen as a complication; a separate variable is constructed which distinguished patients who have a recurrent infarction while in hospital from patients who have only one infarction. The three types of enzyme release for the patient population at hand are presented in tables 6.19-21. 
Table 6.19 Severity categories, indicating myocardial necrosis estimated by total SGOT-release.

Severity category

0
1
2
3
4
5
6

Subtotal of relevant cases

Missing cases

Total

\section{Description}

no sign. SGOT changes

$<1500$ (Un * hours)

$1500-3000$ (U/ * hours)

$3000-6000$ (U// * hours)

$6000-9000$ (U/ * hours)

$9000-12000($ Un * hours $)$

$>12000$ (Un * hours)
Frequencies

\section{2}

83

84

96

46

28

18

Table 6.20 Severity categories indicating myocardial necrosis estimated by total CPK-release.

Severity category

0
1
2
3
4
5

Subtotal of relevant cases

Missing cases

Total

\section{Description}

$$
\begin{aligned}
& \text { no sig. CPK changes } \\
& <10000(\text { Uh * hours) } \\
& 10000-20000(\text { U/ * hours }) \\
& 20000-40000(\text { U/ * hours) } \\
& 40000-60000(\text { Un * hours) } \\
& >60000(\text { Un * hours) }
\end{aligned}
$$

Frequencies

Table 6.21 Severity categories indicating myocardial necrosis measured by the IDII-peak during stay.

Severity category

0
1
2
3
4
5

\section{0}

1

3

4

5

Subtotal of retevant cases

Missing cases

Total
Description

Frequencies

no sign. LDH changes

$<700(\mathrm{U} / \mathrm{l})$

$700-1400(\mathrm{U} / \mathrm{l})$

130

$1400-2100(U / l)$

54

$2100-2800(U / l)$

20

$>2800$ (U/)
85

74

60

56

390

74

464

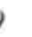




\subsubsection{Severity of necrosis measured by 'irreversible thallium changes'.}

Another method to measure myocardial necrosis is the so-called thallium test (see also $\$$ 6.2.2.3). In necrotic parts of the heart the thallium uptake is severely reduced compared to other parts. Irreversible thallium changes located by 'dark spots' measure myocardial necrosis or sometimes severely injured tissue which possesses a longer' lasting recovery time.

Scores on the thallium test for each wall segment are: $(0)$ no irreversile thallium changes and (1) presence of irreversible thallium changes.

The extent of myocardial necrosis.

It is assumed that severity of myocardial necrosis will increase, if irreversible thallium changes are extended over more wall segments. The number of wall segments with irreversible thallium changes is used as an indicator for the extent of myocardial necrosis. The number of wall segments with irreversible thallium changes is connected with a severity weight. If no irreversible thallium changes are present, a severity weight of ' 0 ' is given. In case one or two wall segments have irreversible thallium changes, severity is assumed to be equal and patients are given a severity weight, of ' 1 '. When three or more wall segments

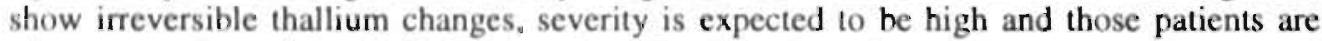
given a severity weight of ' 2 '. The number of patients in each severity category is presented in table 6.22 .

Table 6.22 Severity categories describing the extent of irreversible thallium changes.

Severity category

()

1

2

Subtotal of relevant cases

Missing cases

Total
Description

no irreversible thallium changes

irreversible thallium changes in 1 or 2 wall segments

irreversible thallium changes in 3 wall seg-

ments
Frequencies

22

56

8

The location of myocardial necrosis.

This indicator refers to the location within the left ventricle where irreversible thallium changes may be observed. As discussed before, it is assumed that myocardial necrosis present in the anteroseptal wall segment of the left ventricle is more severe than when present in other wall segments. A severity indicator is constructed to measure the severity of irreversible thallium changes based on their iocation in the left ventricle. If no irreversible thallium changes are present these patients are given a severity weight of ' 0 '. Patients with irreversible thallium changes in the anteroseptal wall segment are given a higher severity weight (severity weight of ' $2{ }^{\circ}$ ) than the remaining patients (severity weight of ' 1 '). The number of patients in each severity category is presented in table 6.23 . 
Table 6.23 Severity categories describing the location of irreversible thallium changes.

Severity category

0

I

2
Description

no irreversible thallium changes

irreversible thallium changes non-anterosep-

tal

irreversible thallium changes anteroseptal
Frequencies

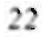

43

21

\subsubsection{Severity of ischemic pain.}

Patients with myocardial ischemia often exhibit prolonged chest pain. Usually, patients with myocardial infarction will experience severe typical pain in the precordial area and radiation to the left shoulder and arm. In some cases the pain will be absent or minor and will be overshadowed by the symptoms of acute complications. The presence or absence of typical ischemic pain is measured for each time period. Subsequently, a trend in ischemic pain is distinguished in the following way (see table 6.24.): patients with no ischemic pain during the entire hospital stay are grouped in severity category ' 0 ' $(\mathrm{n}=55)$; patients suffering from ischemic pain only during the acute phase of the stay (TO and T1) are grouped in severity category '1' $(\mathrm{n}=307)$. Patients with (prolonged) ischemic pain during the acute phase of the stay as well as later on during hospital stay (T2 or T3) are grouped in severity category '2' $(\mathrm{n}=95)$.

Table 6.24 Severity categories describing ischemic pain during stay.

Severity category

\section{0}

2

Subtotal of relevant cases

Missing cases

Total

\section{Description}

no ischemic pain at all

acute ischemic pain only in $T 0$ or $T I$

prolonged ischemic pain in $T 2$ or $T 3$
Frequencies

$\begin{array}{r}55 \\ 307 \\ 95 \\ \hline 457 \\ 7 \\ \hline 464\end{array}$

\subsubsection{Severity of cardiac wall motion abnormalities.}

Due to myocardial injury abnormal wall motion in the cardiac wall segments can occur. The cardiac wall motion is measured usually by echocardiogram. Impaired contraction of specific cardiac segments can be monitored and distinguished, by this technique. Mildly injured zones lose part of their ability to contract (hypokinetic segments). Severely injured segments may fail to contract (akinetic segments) or can bulge in opposite directioni (dyskinetic segments). So, abnormal cardiac wall motion can affect the heart's pumping 
performance (cardiac function: see $\$ 6.2 .8$ ).

By means of echocardiography it is possible to visualize the contractile pattern of the seven left ventricular wall segments: septal, anterior, lateral, posterior, inferior, basal and apical. For each wall segment, four types of wall motion abnormalities are distinguished:

0 : No wall motion abnormalities;

1 : Hypokinetic abnormalities: reduced wall motion;

2: Akinetic abnormalities: absence of wall motion;

3 : Dyskinetic abnormalities: paradoxical wall motion;

The intensity of cardiac wall motion abnormalities.

The intensity (or degree) of left ventricular wall motion abnormalities is measured by the type of segmental wall motion abnormalities. It is obvious that if no wall motion abnormalities are present, patients are categorized in the lowest severity group of ' $\theta$ '. Hypokinetic deviations are assumed to be less severe; patients with only this type of wall motion abnormality are also grouped in severity category ' 0 '. Akinetic or dyskinetic deviations are assumed to be more severe. Patients with either akinetic or dyskinetic or both wall motion abnormalities are assumed to be equal as far as severity is concerned; they are grouped in severity category " $\mathrm{l}$ ". The number of patients within each severity category is presented in table 6.25 .

Table 6.25 Severity patterns and categories describing the intensity in cardiac wall motion abnormalities during total hospital stay.

\begin{tabular}{|c|c|c|c|c|}
\hline $\begin{array}{l}\text { Severity } \\
\text { pattern }\end{array}$ & Description & Freq. & $\begin{array}{l}\text { Severity } \\
\text { category }\end{array}$ & $\begin{array}{r}\text { Combined } \\
\text { freq. }\end{array}$ \\
\hline$a$ & no wall motion abnormalities & 22 & & \\
\hline$b$ & hypokinetic wall motion & 62 & 0 & 84 \\
\hline$c$ & akinetic or dyskinetic wall motion & 156 & & \\
\hline$d$ & akmetic and hypokinetic wall motion & 61 & $l$ & 232 \\
\hline$e$ & $\begin{array}{l}\text { akinetic and dyskinetic (and hypoki. } \\
\text { netic) wall motion }\end{array}$ & 15 & & \\
\hline \multicolumn{2}{|c|}{ Subrotal of relevant cases } & 316 & & 316 \\
\hline \multicolumn{2}{|c|}{ Missing cases } & 148 & & 148 \\
\hline \multicolumn{2}{|l|}{ Total } & 464 & & 464 \\
\hline
\end{tabular}

The extent of cardiac wall motion abnormalities.

The extent of wall motion abnormalities refers to the number of wall segments with any kind of wall motion abnormality. As mentioned above, seven wall segments are distinguished and it is assumed that the severity of abnormal wall motion would increase when the number of wall segments with abnormality increases. If no wall motion abnormalities are present. patients will get a severity weight of "0". Patients, with wall motion abnormalities in one or two wall segments are considered to be equivalent as far as severity is concerned. They' get. a severity weight of ' 1 '. If there are three or four wall segmenis with wall motion abnormalities, a severity weight of ' 2 '. respectively ' 3 ' is given. Patients with wall motion abnormalities in five wall segments are given a severity weight of ' 4 '. Due to small numbers, patients with wall motion abnormalities in six or seven wall segments are recoded into the same category group and obtain a score of ' 5 '. The number of patients in each severity category is presented in table 6.26. 
Table 6.26 Severity categories describing the extent of cardiac wall motion abnormalities during total hospital stay.

\begin{tabular}{clr}
\hline Severity category & Description & Frequencies \\
0 & no wall motion abnomalities & 22 \\
$I$ & wall motion abnomalities in 1 or 2 walls & 126 \\
2 & wall motion abnormalities in 3 walls & 89 \\
3 & wall motion abnormalities in 4 walls & 40 \\
4 & wall motion abnormalities in 5 walls & 22 \\
5 & wall motion abnormalities in 6 or 7 walls & 17 \\
Subtotal of relevant cases & & 316 \\
Missing cases & & 148 \\
Total & & 464 \\
\hline
\end{tabular}

The location of cardiac wall motion abnormalities.

This severity dimension refers to the expected vulnerability of any kind of abnormality in the left ventricle. As discussed before abnormalities in the anteroseptal segments are thought to be more severe than abnormalities in the other segments, regardless of the extent or the intensity (degree) of kinetic abnormalities. Patients with anteroseptal wall motion abnormalities are placed in a higher severity category with a severity weight of ' 2 '. Patients with wall motion abnormalities in other segments of the left ventricle are given a severity weight of ' 1 '. Patients with no wall motion abnormalities at all are given a severity weight of ' 0 " (table 6.27.).

Table 6.27 Severity categories describing the location in cardiac wall motion aboormalities during total hospital stay.

Severity category

0

l

2

Subtotal of relevant cases

Missing cases

Description

no wall motion abnormalities.

non-anteroseptal wall motion abnomalities

anteroseptal wall motion abnormalities

Frequencies

Total

\subsubsection{Severity of cardiac rhythm disorders.}

Patients with myocardial infarction are vulnerable of getting sudden, unexpected disturbances in heart rhythm. A cardiac arrhythmia is defined as a deviation of the 'normal' rhythm of the heart. Rhythm disorders are usually recognized by specific ECGabnormalities. Both, myocardial ischemia as well as myocardial necrosis can cause rhythm disorders. Rhythm disorders may be present within the normal pacemaker cells in the sinus node or AV node, in parts of the conduction system or in the remaining tissue of the atria and ventricles. 
For every hospital period five types of cardiac rhythm disorders were recorded (see table 6.28 ) as (0) not present and (1) present.

\section{Severity indicators.}

Each type of cardiac rhythm disorder is given a severity weight representing the seriousness of the rhythm disorder with regard to morbidity and mortality. It is assumed that the severity of particular cardiac rhythm disorders will differ depending on time of occurrence during the stay in hospital. A difference in severity weights is made between cardiac rhythm disorders during the acute phase (time periods T- 0 and T-1) and the follow-up phase (time periods T-2 and T-3). The severity weights for each cardiac rhythm disorder specified for the period of incidence are presented in table 6.28.

Table 6.28 Severity weight of different cardiac rhythm disorders in relation to the phase of stay.

Type of Cardiac rhythm disorder

No relevani cardiac rhythm disorder

Sinustachycardia

Atrial fluter/-fibrillations

Non-sustained ventricular achycardia

Sustained ventricular tachycardia

Ventricular flutter/-fibrillations
Acute phase (TO/T)

Follow-up phase (T2/T3)

* seldomlv present in TI. mainlv present in TO

The severity of cardiac thythm disorders is measured by the most severe rhythm disorder occuring during the acute phase, respectively the follow-up phase of the stay. This means that if two or more different rhythm disorders are present only the most severe the one with the highest severity weight) is taken into account. It is expected that the rhythm disorder with the highest severity weight will be sufficient in measuring the overall severity of cardiac rhythm disorders.

\section{Severity categories.}

The severity categories are determined by the type of rhythm disorders and the appearance of these disorders in the acute phase or the follow-up phase. A multivariate analysis giving insight into the patterns of appearance of these characteristics provides many unique combinations of cases. Because of the uniqueness of each of these severity patterns, no rules, can he established to combine the many 'pattern' groups of rhythm disorders into one ordinal dimension or scale. All ten categories of rhythm disorders that occur in our population are presented in table 6.29 as a nominal scale (a to j). A large group of patients $(n=222)$ have no rhythm disorder at all during hospital stay; they are allocated to one severity category 'a'. The next largest group of patients with rhythm disorders concerns 
patients with sinustachycardia, exclusively within the first three days of the infarction $(n=105)$; they are grouped into the nominal category ' $b$ '. Patients with ventricular flutter/fibrillations, exclusively within three days of the infarction $(n=18)$ are grouped in severity category 'd'. Patients with atrial flutter/-fibrillations are divided over three different severity categories: patients with this arrhythmia, exclusively within the firs! three days of infarction ('c', $n=21$ ), patients with this arrhythmia within the first three days of infarction combined with sinustachycardia or non-sustained ventricular tachycardia in the follow-up phase ('g'. $\mathrm{n}=6$ ) and patients with recurrent atrial flutter/-fibrillations (first three days and later on) (' $h$ ', $\mathrm{n}=31$ ).

Patients having sinustachycardia or non-sustained ventricular tachycardia in a later phase of the infarction without any previous arrhythmia. $\left(\mathrm{e}^{\prime}, \mathrm{n}=18\right)$ are distinguished from patients with recurrent sinustachycardia or non-sustained ventricular tachycardia during hospital stay ('f, $n=26$ ). Patients with ventricular flutter/-fibrillations during the first days of the infarction combined with atrial futter/-fibrillations or sinustachycardia later on ('i', $n=7$ ) are distinguished from patients who had sinustachycardia or atrial flutter/-fibrillations in the beginning and still had ventricular flutter/-fibrillations or sustained ventricular tachycardia later on ('j', n=9).

Table 6.29 Severity categories describing type and time of appearance of cardiac rhythm disorders.

Nominal severity category

$a$
$b$
$c$
$d$
$e$
$f$
$g$

$h$
$i$
$j$

Subiotal of relevant cases

Missing cases

Total

\section{Description}

no rhythm disorder

acute sinustachycardia

acute atrial fluter/-fibrillations

acute ventricular flutter/-fibrillations

sinustachycardia or non-sustained ventricular

tachycardia in follow-up phase

recurrent sinustachycardia or non-sustained

ventricular tachycardia

acute $A F / F$ combined with sinustachycardia or

non-sustained ventricular tachycardia in foliow.

up phase

recurrent $A F / F$

acute $V F / F$ combined with $A F / F$ or sinustachy-

cardia in follow-up phase

acute sinustachycardia or AF/F combined with

$V F / F$ or sustained ventricular tachycardia in fol-

low.up phase
Frequencies

222

105

21

18

18

26

6

31

$A F / F=$ Atrial Flunter/.Fibrillations

$V F / F=$ Ventricular Flutter/-Fibrillations 


\section{Severity Categories.}

Conduction disorders during the first three days of the stay of AMI-patients have a medical significance that differs from conduction disorders after three days of the infarction. This important notion is taken into consideration when looking at the trends in conduction disorders for the patient population at hand. A rough distinction is made in acute conduction disorders (during the first three days of stay) and conduction disorders in the follow-up phase (T2/T3). The different types of conduction disorders in our patient population have been listed. There appear to be seven different categories of conduction disorders (table 6.31). Patients with no relevant conduction disorder during hospital stay $(n=372)$ are grouped in severity category 'a'. Patients with only Mobitz I AV-block during the first three days after infaretion $(\mathrm{n}=11)$ are grouped together in severity category 'b'. Patients with Mobitz II AV-block or a third degree block with nodal escape rhythm exclusively during the acute phase are grouped in category ' $\mathrm{c}$ ' $(\mathrm{n}=36)$. Patients with an acute right bundle branch block ( $\mathrm{n}=11$, also including a few patients who have the same conduction disorder after three days) are grouped in severity category 'd'. Patients with third degree block with ventricular escape rhythm exclusively during the first three days of infarction are placed in one severity category ('e', $\mathrm{n}=12$ ). If patients have intermittent (recurrent) right bundle branch block, they are grouped into a separate severity category (' $\mathrm{f}, \mathrm{n}=18$ ). Patients who have a third degree block with ventricular escape rhythm after three days in hospital (after having conduction disorders during the first three days of infarction as well) are grouped in another severity category ('g', $\mathrm{n}=4$ ).

Table 6.31 Severity categories describing type and time of appearance of cardiac conduction disorders.

Nominal severity category Description

Frequencies

$\begin{array}{llr}a & \text { no conduction disorder } & 372 \\ b & \text { acute Mobitz I AV-block } & 11 \\ c & \begin{array}{l}\text { acute Mobitz II AV-block or acute third de- } \\ \text { gree block with nodal escape rhythm } \\ \text { acute right bundle branch block }\end{array} & 36 \\ \text { acute third degree block with ventricular esca- } & 11 \\ \text { e } & \begin{array}{l}\text { pe rhythm } \\ \text { recurrent right bundle branch block } \\ \text { third degree block combined with ventricular } \\ \text { escape rhythm in follow-up phase }\end{array} & 12 \\ & \end{array}$

\subsubsection{Severity of heart failure.}

The main function of the heart is to provide an adequate amount of blood for the various body tissues. Heart failure is a condition in which the heart is unable to pump the required amounts of blood into the circulatory system. Heart failure can be restricted to either the left or the right ventricle of the heart.

By means of echocardiography the Left Ventricular Ejection Fraction (LVEF) and the Left Ventricular End Diastolic Dimension (LVEDD) can be obtained indicating the severity of left ventricular failure. The collapse of Vena Cave Inferior (VCI) can be used as a severity indicator for the pumping capacity of the right ventricle. 


\section{Severity Categories.}

Conduction disorders during the first three days of the stay of AMI-patients have a medical significance that differs from conduction disorders after three days of the infarction. This important notion is taken into consideration when looking at the trends in conduction disorders for the patient population at hand. A rough distinction is made in acute conduction disorders (during the first three days of stay) and conduction disorders in the follow-up phase (T2/T3). The different types of conduction disorders in our patient population have been listed. There appear to be seven different categories of conduction disorders (table 6.31). Patients with no relevant conduction disorder during hospital stay $(n=372)$ are grouped in severity category 'a'. Patients with only Mobitz I AV-block during the first three days after infaretion $(\mathrm{n}=11)$ are grouped together in severity category 'b'. Patients with Mobitz II AV-block or a third degree block with nodal escape rhythm exclusively during the acute phase are grouped in category ' $\mathrm{c}$ ' $(\mathrm{n}=36)$. Patients with an acute right bundle branch block ( $\mathrm{n}=11$, also including a few patients who have the same conduction disorder after three days) are grouped in severity category 'd'. Patients with third degree block with ventricular escape rhythm exclusively during the first three days of infarction are placed in one severity category ('e', $\mathrm{n}=12$ ). If patients have intermittent (recurrent) right bundle branch block, they are grouped into a separate severity category (' $\mathrm{f}, \mathrm{n}=18$ ). Patients who have a third degree block with ventricular escape rhythm after three days in hospital (after having conduction disorders during the first three days of infarction as well) are grouped in another severity category ('g', $\mathrm{n}=4$ ).

Table 6.31 Severity categories describing type and time of appearance of cardiac conduction disorders.

Nominal severity category Description

Frequencies

$\begin{array}{llr}a & \text { no conduction disorder } & 372 \\ b & \text { acute Mobitz I AV-block } & 11 \\ c & \begin{array}{l}\text { acute Mobitz II AV-block or acute third de- } \\ \text { gree block with nodal escape rhythm } \\ \text { acute right bundle branch block }\end{array} & 36 \\ \text { acute third degree block with ventricular esca- } & 11 \\ \text { e } & \begin{array}{l}\text { pe rhythm } \\ \text { recurrent right bundle branch block } \\ \text { third degree block combined with ventricular } \\ \text { escape rhythm in follow-up phase }\end{array} & 12 \\ & \end{array}$

\subsubsection{Severity of heart failure.}

The main function of the heart is to provide an adequate amount of blood for the various body tissues. Heart failure is a condition in which the heart is unable to pump the required amounts of blood into the circulatory system. Heart failure can be restricted to either the left or the right ventricle of the heart.

By means of echocardiography the Left Ventricular Ejection Fraction (LVEF) and the Left Ventricular End Diastolic Dimension (LVEDD) can be obtained indicating the severity of left ventricular failure. The collapse of Vena Cave Inferior (VCI) can be used as a severity indicator for the pumping capacity of the right ventricle. 


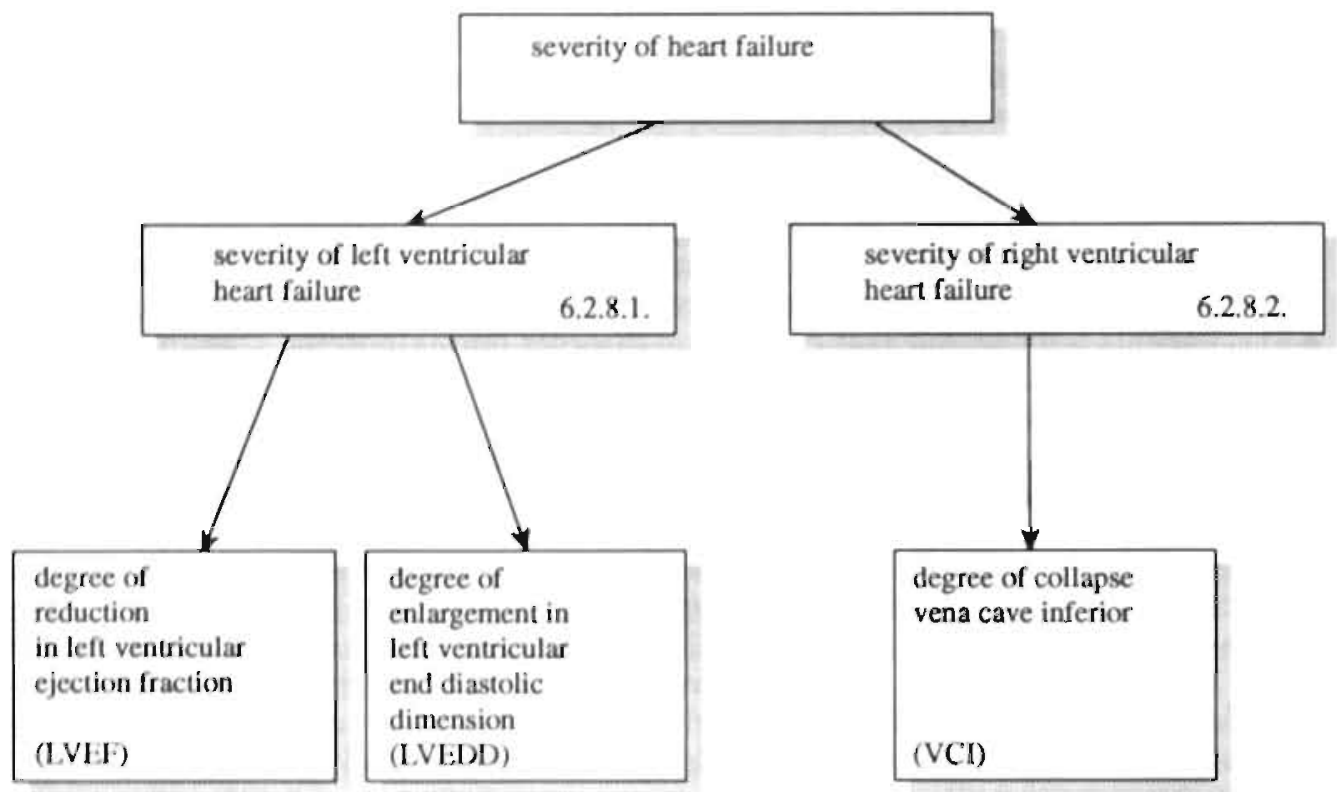

\subsubsection{Severity of heart failure in the left ventricle.}

Left Ventricular Ejection Fraction (LVEF).

Measurement of the LVEF by echocardiogram is in most cases periormed only once during hospital stay. For the entire patient population at hand only 7 patients had two echocardiograms; in those cases the last score is used. The LVEF measurement is a pereentage-index normally (in healthy persons) ranging between $40 \%$ and $70 \%$. If the LVEF decreases the severity of heart failure increases. The results of the LVEF are categorized into severity groups, illustrated in table 6.32. In total, 141 patients have no LVEF-score because of missing data or mostly because echocardiography has not been applied during hospital stay. Most patients $(\mathrm{n}=183$ ) have a 'normal' LVEF-value. In patients with impairment of pumping function, 81 showed a slight impairment, 50 patients showed a severe impairment and 9 patients showed a very severe impairment.

Table 6.32 Severity categories describing heart failure measured by the left Ventricular Ejection Fraction.

Severity category

0
1
2
3

Subtotal of relevant cases

Missing cases
Description

Frequencies

normal ( $L V E F>40 \%)$

slightly impairment $(30 \%<L V E F<40 \%)$

severe impaiment $(20 \%<L V E F<30 \%)$,

very severe impairment ( $L V E F<20 \%)$.
183

81

50

9 


\section{Left Ventricular End Diastolic Dimension (LVEDD).}

The second indicator to measure left ventricle heart failure is the Left Ventricular End Diastolic Dimension (LVEDD). This is the largest diameter at the end of the diastolic phase (expressed in millimeters). It is assumed that severity of heart failure will increase if the LVEDD increase. Patients are categorized into two severity' groups: '0' patients with 'normal' LVEDD of less than $55 \mathrm{~mm}(\mathrm{n}=282)$, and ' 1 ' patients with deviating LVEDD of more than $55 \mathrm{~mm}(\mathrm{n}=36)$.

\subsubsection{Severity of heart failure in the right ventricle.}

The pumping capacity of the right ventricle can be measured by the percentage of collapse in the Vena Cava Inferior (VCI-index). In normal conditions the VCI-index is expected io be higher than $50 \%$. The percentages of the VCI collapse are categorized into two severity groups: ' 0 ", patients having 'normal' VCI of more than $50 \%(n=281)$ and ' 1 ', patients with deviating VCI of less than $50 \%(n=37)$. During hospital stay, 18 patients show abnormal wall motion in the right ventricle while 76 patients appear to have significant ST-changes in the right ventricle as indicated by ECG-leads.

\subsubsection{Severity of physical deficiencies and specific cardiac complications.}

Specific cardiac complications relevant in the context of this study are: mitral insufficiency $(n=60)$, cardiac shock $(n=48)$ and pericarditis $(n=43)$. The previous cardiovascular and cardiac deficiencies $(\$ 6.2 .1-\$ 6.2 .8)$ will directly or indirectly cause specific deficiencies in other organs. In some cases deficiencies (measured by $0-1$ scores) like palpitations $(n=24)$. pre-collapse $(n=36)$, syncope $(n=39)$ and Cerebral Vascular Accident (CVA)/Transient Ischemic Attack (TIA) $(n=9)$ may occur. Consequences of left-sided heart failure like pulmonary edema (tabie 6.33) and dyspnea $(n=115)$, may be present. Right-sided heart failure can lead to hepatomegaly $(n=26)$, leg edema and ascites (table 6.34). Furthermore, a patient's condition may be determined by observing his or her blood pressure. Low blood pressure (systolic bp $<90 \mathrm{~mm}$ of mercury) is measured in terms of four severity categories: ' 0 ', no low blood pressure $(n=306)$, ' 1 ', low blood pressure in only one time period $(n=97)$, ' 2 '. low blood pressure in two time periods $(n=44)$, ' 3 ' sustained low blood pressure in three or four time periods $(\mathrm{n}=17$ ). High blood pressure (systolic bp $>150 \mathrm{~mm}$ of mercury) is also measured in terms of four severity categories: '0', no high blood pressure $(n=352)$, '1', high blood pressure in only one time period: $(n=77)$, ' 2 ', high blood pressure in iwo time periods $(n=22), 3^{\prime}$ ' sustained high blood pressure in three or four time periods $(n=13)$. Myocardial infarction may be followed by Angina Pectoris (A.P). The New York Heart Association (NYHA) has developed an international standard to measure AP given different stages of exertion. For the patient population, patients are categorized according to the NYHA-index into : I; no A.P ( $\mathrm{n}=304$ ), II; AP during heavy physical exertion ( $\mathrm{n}=\mathrm{I})$; III; AP during minor or moderate exertion $(n=12)$ and IV; AP at rest $(n=138)$. 
Table 6.33 Severity' categories deseribing lung edema in AMI patients during stay.

Severify category

$\begin{array}{ll}0 & \text { no lung edema } \\ 1 & \text { minor lung edema } \\ 2 & \text { moderate lung edema } \\ 3 & \text { severe lung edema }\end{array}$

Subtotal of relevant cases

Missing cases

Total
Description

no lung edema

moderate lung edema

severe lung edema
Frequencies

355

59

29

18

461

3

464

Table 6.34 Severity categories describing body edema in AMI patients during stay.

Severity category

0

l

2
Description

no edema

edema in ankles or feel

edema in legs or abdomen
Frequencies

443

16

5 
In table 6.35 descriptive statistics of all severity indicators as described in this chapter are presented.

Table 6.35 Descriptive statistics on indicators of severity of illness in AMI patients during stay $(86 \leq \mathrm{n} \leq 464)$.

\begin{tabular}{|c|c|c|c|c|c|}
\hline Variable & Legend & $\begin{array}{l}\text { Category } \\
\text { Range }\end{array}$ & Mean & Siddev & Freq. \\
\hline STENINT & coronary stenosis intensity/degree & 0.5 & 3.12 & 1.74 & 182 \\
\hline STENEXT & coronary stenosis extent & $0-4$ & 2.12 & 1.12 & 182 \\
\hline STENLOC & coronary stenosis location & $0-5$ & 2.58 & 1.51 & 178 \\
\hline ISEXT & ischemia extent ( $E C G$ ) & $0-3$ & 1.57 & .73 & 462 \\
\hline ISLOC & ischemia location ( $E C G$ ) & $0-3$ & 1.31 & .60 & 462 \\
\hline BRINT & ischemia intensity (Brice) & $0-4$ & 1.75 & 1.47 & 203 \\
\hline BREXT & ischemia extent (Bruce) & $0-3$ & $.83^{\prime}$ & .75 & 203 \\
\hline BRLOC & ischemia location (Bruce) & $0-2$ & $.82^{\prime}$ & .69 & 203 \\
\hline BREXP & $\begin{array}{l}\text { ischemia expansion (additional ischemia, } \\
\text { Bruce) }\end{array}$ & $0-2$ & 1.08 & .88 & 203 \\
\hline ISTHEXT & ischemia extent (thallitum) & $0-1$ & .22 & .42 & 86 \\
\hline ISTHLOC & ischemia location (thallinm) & $0-2$ & .26 & .51 & 86 \\
\hline NECEXT & necrosis extent (ECG) & $0-2$ & 1.04 & .53 & 462 \\
\hline NECLOC & necrosis location (ECG) & $0-2$ & 1.16 & .62 & 462 \\
\hline SGOT & SGOT-enzyme release & $0-6$ & 2.72 & 1.43 & 357 \\
\hline$C P K$ & CPK-enzyme release & $0-5$ & 2.75 & 1.39 & 390 \\
\hline$\angle D H$ & LDH-peak value & 0.5 & 1.83 & 1.00 & 408 \\
\hline NETHEXT & necrosis extent (thallium) & $0-2$ & .84 & .57 & 86 \\
\hline NETHLOC & necrosis location (thallitm) & 0.2 & .99 & .71 & 86 \\
\hline ISPAIN & ischemic pain & $0-2$ & 1.09 & .57 & 457 \\
\hline CWMINT & intensity of cardiac wall motion abnormality & $0-4$ & 1.95 & .93 & 316 \\
\hline CWMEXT & extent of cardiac wall motion abnormality & $0-5$ & 1.89 & 1.25 & 316 \\
\hline CWMLOC & location of cardiac wall motion abnormality & $0-2$ & 1.34 & .60 & 316 \\
\hline RHYTHM & cardiac rhythm disorders & 0.9 & - & - & 463 \\
\hline CONDUC & cardiac conduction disorders & 0.6 & - & - & 464 \\
\hline LVEF & left ventricular ejection fraction & $0-3$ & .64 & 84 & 323 \\
\hline LVEDD & left ventricular end diastolic dimension & $0-1$ & 11 & 32 & 318 \\
\hline $\mathrm{VCl}$ & collapse vena cava inferior & 0.1 & .12 & .32 & 318 \\
\hline ISCRV & ischemia in right ventricle & $0-1$ & .17 & .38 & 436 \\
\hline CWMRV & $\begin{array}{l}\text { cardiac wall motion abnormality in right } \\
\text { ventricle }\end{array}$ & $0-1$ & .06 & .23 & 316 \\
\hline$P A L P$ & palpitations & $0-1$ & .05 & .22 & 462 \\
\hline$D Y S P N$ & dyspnea & $0-1$ & .25 & .43 & $\$ 62$ \\
\hline PRECOL & precollapse & $0-1$ & .08 & .27 & 463 \\
\hline SYCOL & syncope/collapse & $0-!$ & .08 & .28 & 453 \\
\hline HBLPRES & high blood pressure & $0-3$ & .51 & .81 & 464 \\
\hline LBLPRES & lowblood pressure & $0-3$ & .34 & .70 & 464 \\
\hline$A P$ & NYHA-AP index & $0-3$ & .96 & 1.38 & 455 \\
\hline LEDEMA & lung edema & $0-3$ & .37 & .77 & 461 \\
\hline BEDEMA & body edema & $0-2$ & .06 & .27 & 454 \\
\hline HEPTO & hepatomegaly & 0.1 & .06 & .23 & 461 \\
\hline PERIC & pericarditis & 0.1 & .09 & .29 & 464 \\
\hline MITINS & mitral insufficiency & $0-1$ & .13 & .34 & 464 \\
\hline CSHOCK & cardiogenic shock & 0.1 & .10 & .30 & 464 \\
\hline$C V A$ & CVATTIA & $0-1$ & .02 & .14 & 464 \\
\hline
\end{tabular}




\subsection{Background characteristics of patients.}

Individual background characteristics may be related to a patient's severity of illness during hospital stay (6.3.1). Specific contra-indications for medical treatment may also be related to medical care costs (6.3.2). Next to individual background characteristics the enrollment of patients in a research protocol is taken into account (6.3.3).

\subsubsection{Individual background characteristics.}

It might be expected that severity of myocardial infarction will vary according to typical patient characteristics. For instance older patients are assumed to be different with regard to severity than younger patients. In addition, specific risk factors for cardiovascular disease and the patient's medical history are thought to be relevant.

The patiene's background characteristics can be distingtished into six categories:

(1) demographic patient characteristics; this concerns the patient's age and sex.

(2) risk factors; these are factors that might be related to myocardial infaretion (like smoking , high blood pressure, diabetes etc.).

(3) cardiovascular medical history; this concerns cardiovascular problems before the current hospital admission which might be related to the severity of the myocardial infarction during hospital stay.

(4) cardiovascular therapeutic history; this covers previous medical interventions with respect to cardiovascular problems, before the current hospital admission of the patient.

(5) non-cardiovascular medical history; this çoncerns former medical, non-cardiovascular complications.

Data on variables belonging to these five categories of background characteristics have been collected using the patient's medical record. Registration of these variables did result mostly in dichotomous variables. For the patient population at hand, results are presented in table 6.36. As can be seen from this table, risk factors like smoking and positive family history with regard to heart diseases affect almosi half of the patients. Nearly $50 \%$ of the patients had angina pectoris before hospial admission, $28 \%$ suffered from hypertension and $11 \%$ were known to have coronary insufficiency. In $25 \%$ of the cases patients suffered from (one or more) previous infarction(s). To a lesser degree, patients had thythm disorders (8\%), peripheral and cerebral atherosclerosis ( $9 \%$ and $6 \%$ resp.) or heart failure $(5 \%)$. Other cardiovascular deficiencies had occurred infrequently: $5 \%$ of all patients underwent coronary bypass surgery and $3 \%$ had received thrombolytic therapy. PTCA and pacemaker implantation was performed in $1 \%$ of the patient population. In $9 \%$ of the cases respiratory problems (COPD), in $4 \%$ kidney disorders and in only $1 \%$ liver disorders were present as non-cardiovascular deficiencies. 
Table 6.36 Descriptive Statistics on the patient's background characteristics.

I Individual patient characteristics:

- age Mean: 62.73 Std.dev.: 11.64 Min.: 31 Max.: 91

- sex Men: $70.0 \%$ Women: $30.0 \%$

2 Risk factors:

- family medical history (heart diseases)

Mean* Std.Dev, N

- smoking behaviour

- diaberes mellirus

$.42 \quad .49 \quad 387$

- disorders of lipometabolism

- other risk factors

$.49 \quad .44 \quad 449$

$.10 \quad .30 \quad 463$

$\begin{array}{lll}.05 & .21 & 463\end{array}$

$.26 \quad .44 \quad 449$

3 Cardiovascular medical history:

- angina pecioris

- hypertension

- old myocardial infarction

- coronary insufficiency

- peripheral arteriosclemsis

- cerebral arteriosclerosis

- cardiac rhythm disorders

- heari failure

- valvular heart defect( $s$ )

- cardiac conduction disorders

- cardiomyopathy

- congenital heart disorders

- other (pericarditis, mitral insufficiency, etc)

$\begin{array}{lll}.48 & .25 & 462\end{array}$

$\begin{array}{lll}.28 & .45 \quad 463\end{array}$

$.25 \quad .43 \quad 462$

$.11 \quad .32 \quad 463^{\prime}$

$.09 \quad .29 \quad 460$

$.06 \quad .24 \quad 461$

$\begin{array}{lll}.08 & .28 & 458\end{array}$

$\begin{array}{lll}.05 & .23 & 459\end{array}$

$.03 \quad .17 \quad 458$

$.01 \quad .11 \quad 458$

$\begin{array}{lll}.01 & .09 & 458\end{array}$

$.00 \quad .00 \quad 458$

$\begin{array}{lll}.02 & .15 \quad 457\end{array}$

4 Cardiovascular therapeutic history:

- coronary bypass surgery

- thrombolytic therapy

- pacemaker

- PTCA

- intra-aortic balloon counterpulsation

$05 \quad .2 ! \quad 46 !$

$03 \quad .17 \quad$ का

$01 \quad .08 \quad 461$

$.01 \quad .09 \quad 401$

$.00 \quad .00 \quad 461$

5 Non-cardiovascular medical history:

- COPD

$\begin{array}{lll}.09 & .29 & 463 \\ .04 & .20 & 463 \\ .01 & .09 & 463 \\ .06 & .24 & 458\end{array}$

* The mean yalue is the proporion of relevant cases scoring 'positively' on the background characteristic. 


\subsubsection{Contra-indications.}

Contra-indications to diagnostic or therapeutic services are very important in explaining decision-making in therapy and costs of treatment. It is quite clear that one treatment of patients having an acute myocardial infarction is very much influenced by specific contraindications. This treatment concerns thrombolytic therapy. In 1987-1988 at the Department of Cardiology in Maastricht thrombolytic therapy was normally not given to patients older than 75. Furthermore if the duration of ischemic pain before the moment of admission was more than six hours, or if patients were in coma also no thrombolytic therapy was given. The rules according to which decisions are made may' differ from hospital to hospital, from one country to another or from year to year. In our patient population 78 patients are older than 75 years, 52 patients had ischemic pain more than 6 hours before hospital admission and 11 patients were in coma on the moment of admission.

\subsubsection{Enrollment of patients in a hospital research program.}

A patient's involvement in a scientific research protocol during his or her stay in the hospital is also recorded. In the University Hospital of Maastricht projects for scientific and/or educational purposes are quite normal. If patients have been subject to a specific research protocol of a clinical trial involving specific patient care, this characteristic is specially recorded. One important research project was taking place during the period of investigation; it concerns a multi-center clinical trial to compare the efficacy of the experimental thrombolytic drug 'pro-urokinase' to the standard drug 'streptokinase' (PROurokinase In Myocardial Infarction). Henceforth this clinical trial will be called the PRIMIprotecol, (Meyer, 1989). For the patient population at hand 59 patients had been allocated to participate in this clinical trial. During the acute phase of the stay all patients involved in this trial did receive thrombolytic therapy followed by a heart catheterization. 


\section{Investigating hypothesized relations between severity of illness and costs of medical care.}

In this chapter results regarding the relationship between the patient's severity of illness and specific costs of medical care will be presented. Differences in costs observed in the research population at hand will be analyzed. First, methods used in data analysis will be outlined (7.1.). A distinction will be made between the relation of period-specific costs of medical care and the period-specific classifications of severity of illness (see model in figure 3.2.), and the relation of costs of medical care during total hospital stay and the (dynamic) classifications of severity of illness (see model in figure 3.3).

Next, in $\$ 7.2$. a period-specific cost analysis will be presented for costs of medical care made during the acute phase of the stay. Costs of thrombolytic therapy, heart catheterization and PTCA are generally made during this phase of hospital stay. It is assumed that differences in these costs depend on differences in severity of illness during this time period.

The second type of analysis concerns the patient's total hospital stay (\$ 7.3.). It stands to reason that changes in severity of illness may be relevant in explaining length of stay and costs of medical care during total hospital stay. The (dynamic) severity categories defined in chapter 6 are assumed to be more appropriate for this type of analysis. In $\$ 7.3$ the following dependent variables will be analyzed: the length of stay, the costs of the CCU and other costs of medical care (i.e. drugs, laboratory services, electrocardiograms, echocardiograms and exercise tests). Finally, the conclusions will be summarized (\$ 7.4.) and results from this chapter will be used to propose a Severity of Illness Classification (SIC) for patients with Acute Myocardial Infarction (see chapter 8).

\subsection{Methods used in data analysis.}

The main goal of the fortheoming analysis is to investigate which severity criteria have a substantial effect on length of stay or specific costs of medical care.

The multivariate modeís in chapter 3 will be translated into a path-analytic model. Path analysis is a method of causal data analysis: relations between variables are assumed to be causally asymmetric and will correspond to the hypothesized relations between concepts in a theoretical model. In such models one is usually interested in explaining a 'dependent' variable (i.e. specific costs of medical care or length of stay) and the other variables may be seen as the 'independent' variables (i.e. severity indicators) providing the explanation of the variations within the dependent variable. Correlations between the dependent variable and the independent (the explaining) variables can be differentiated into direct effects, indirect effects (via other factors) and other correlational rest relations. The statistical distribution of the dependent variable is usually assumed to be normal and the scale of measurement is at least the interval scale. If the measurement level of the predictors is also interval, relations are usually thoughe to be linear. No interactions are assumed to exist between the causal factors with regard to the dependent variable. In this case a linear regression analysis can be performed.

In this study the dependent (costs) variables are ratio-scales, but the independent (severity) variables will vary from nominal to interval-scales. In such case one can usually perform an analysis of covariance: the effects of the interval variables can be examined after controlling for the nominal variables and vice versa. For instance, the effect of enzyme release (independent interval variable) on length of stay (dependent variable) can be investigated 
separately for men and women (nominal variable). However, if we are interested both in the effects of sex (factor) and enzyme release (covariate) on e.g. the length of stay: a combination of analysis of variance and regression analysis should be performed. This is done in analysis of covariance, which is a combination (a hybrid) between regression analysis and analysis of variance. The analysis of variance-part in analysis of covariance is identical to a procedure referred to as dummy-regression analysis, in which the nominal variables are broken down into so-called dummy variables. One of the advantages of using dummy regression analysis is that all effects of interval as well as nominal independent variables on the dependent variable can be examined within the terminology of regression analysis.

\section{* Dummy-variable construction.}

Severity of illness measured by categories representing nominal variables can be broken down into dummy variables. Dummy variables (or '0- I' type variables) obey the 'simple contrast' assumptions in analysis of variance. One of the advantages of this contrast is that it will discriminate between patients who have no deficiencies ('normal') and patients with clinical deviations from 'normal'. Combinations of K-1 dummy variables will be used when the nominal variable contains $\mathrm{K}$ classes. All dynamic severity categories expressed in a nominal scale can thus be represented by dummy variables. This concerns the categories representing cardiac rhythm disorders (see $\$ 6.2 .6)$ and the categories representing cardiac conduction disorders $(\sec \$ 6.2 .7$.). All other severily categories are assumed to have an order; these categories can also be decomposed to dummy variables. Another advantage of using dummy variables is that patients having missing values on the severity indicators can be represented by a special dummy variable. This way, the total patient population can be included in the regression analysis and an unneccessary reduction in the number of patients can be avoided in data analysis. These missing categories concern the severity categories of coronary stenosis, myocardial ischemia measured by Bruce tests and thallium exercise tests, myocardial necrosis measured by enzyme rates and thallium exercise test, ischemic pain, cardiac wall motion abnormalities, the LVEF and specific physical deficiencies. All dummy variables used in the forthcoming analyses are described in appendix 16.

\section{* Procedures in data analysis.}

Firsi, all correlations between dependent and independent variables are screened for statistical significance. All independent variables having statistically significant correlations with the dependent variable in question will be presented in a corrclation matrix. In order to reject null hypotheses involving effects in the regression model a $95 \%$ probability level of statistical significance is maintained. In case of nominal variables first a "one-way" analysis of variance is made and $e^{2}$ is calculated. Subsequently, each variable will be tested for significant effects on the dependent variable. A general linear regression model will be applied using the least squares estimation (Schroeder e.a. 1986, Fox, 1991). The regression analysis is made by introducing blocks of independent variables. Variables having a significant effect in an analysis will be included into a next step in which a new block of variables is tested. The process will be repeated until all possible effects have been investigated. The eventual regression analysis will contain only those variables having a significant effect on the dependent variable. Only the results of the eventual regression analysis model will be presented in the fortheoming paragraphs. Of course, if one contrast in the $\mathrm{K}-1$ set of dummy variables belonging to a nominal variable with $\mathrm{K}$ categories appears to be statistically significant, the whole set will have to be included. All statistically significant effects will be interpreted and presented in a path-analytic diagram showing the relative importance of the variables (Beta-weights). As mentioned before: in multivariate data analysis values on indicators of cases may be missing for specific or unclear reasons (no test done or lost tesi scores). Several solutions may be applied to overcome this problem 
of missing data. The most valid. solution is to eliminate all cases that have a missing value for any variable considered relevant in analysis. This is sometimes called listwise deletion of missing cases". Another solution is to compute measures of association for pairs of variables separately which will result in different numbers of cases used for each measure. This latter way is called 'pairwise deletion of missing cases'. In the data analysis of this text a. listwise deletion of missing cases will always be applied. This procedure could only be applied because we have used the possibilities of defining 'missing category' dummy variables.

A typical problem associated with data used in a regression analysis is (multi)collinearity; it arises when two or more variables used as predictors in a regression function are highly correlated. In such a case the estimated parameters may be incapable of sorting out the independent effects of each predictor on the dependent variable. In our data matrix (multi)collinearity may appear in the variables that represent the severity dimensions of one and the same deficiency (intensity, extent and location). Therefore in the analysis each of the severity dimensions will be introduced in the analysis separately from both others to avoid (multi)collinearity.

Another problem in regression analysis is heteroskedasticity in the variance of dependent variable: this variance will tend to increase (or sometimes decrease) given the values of the predictor. Transformations of the dependent variable may be performed to deal with heteroskedasticity (for instance a $\log$ transformation). However given the explorative character of this study this type of solution for this problem has not been made in the forthcoming analysis. We assume that the results of the analysis will not differ very much when using a transformation. Another reason is that using a transformation will make the interpretation of regression coefficients somewhat more difficult. To avoid heteroskasticity as much as possible, many confounding factors will be introduced in regression analysis and also a residual analysis plus outlier identification will be made. An outlier identification is applied after the eventual model results have been determined. Patients with extremely high residual $z$-scores in their predicted values on the dependent variable are then removed from further analysis. These outliers will be removed from analysis for technical reasons only: their extreme scores on the dependent variable tend to have a disturbing influence on the slope of the regression paramelers.

\section{* The effect of severity of illness.}

The central hypothesis in this study is described in chapter 3. We will repeat this hypothesis here and place it within the context of the model of explanation of diagram 3.3. The length of a hospital stay and the costs of medical care of AMI patients are assumed to be explained by the patient's severity of illness during hospital stay. It is assumed that patients with a severe condition will stay in hospital for a longer period of time and that they will have higher costs ol medical care.

As we have seen in chapters 5 and 6 several cardiac and cardiovascular deficiencies can be measured in patients with acute myocardial infarction. The severity of each cardiac and cardiovascular deficiency can be measured by means of one or more severity indicators and each indicator can in turn be specified inte one or more severity dimensions (intensity. extent or location). The assumed effects of the categories reflecting severity of illness on length of stay and medical costs are described in path-diagram 7.1. This diagram can be seen as an elaboration of diagram 5.6. As can be seen in this diagram, the severity of the medical deficiencies may cause direct or indirect effects on the costs of hospital care. For instance one of the direct effects is that patients with severe heart, failure will have higher costs of hospital care; an indirect effect may result from one of the causes of heart failure, such as specific cardiac rhythm conduction disorders. However, these causes of heart failure may also directly affect the costs of medical care as well. It is to be expected that not all 
deficiencies are 'important' in explaining (specific) costs of medical care. The question then is which severity indicators are really relevant in explaining medical care costs and furthermore what the relative importance of each indicator is compared to other indicators.

Diagram 7.1 Causal relations between indicators of severity of illness and costs of hospital medical care in patients having AMI (see also diagram 5.6).

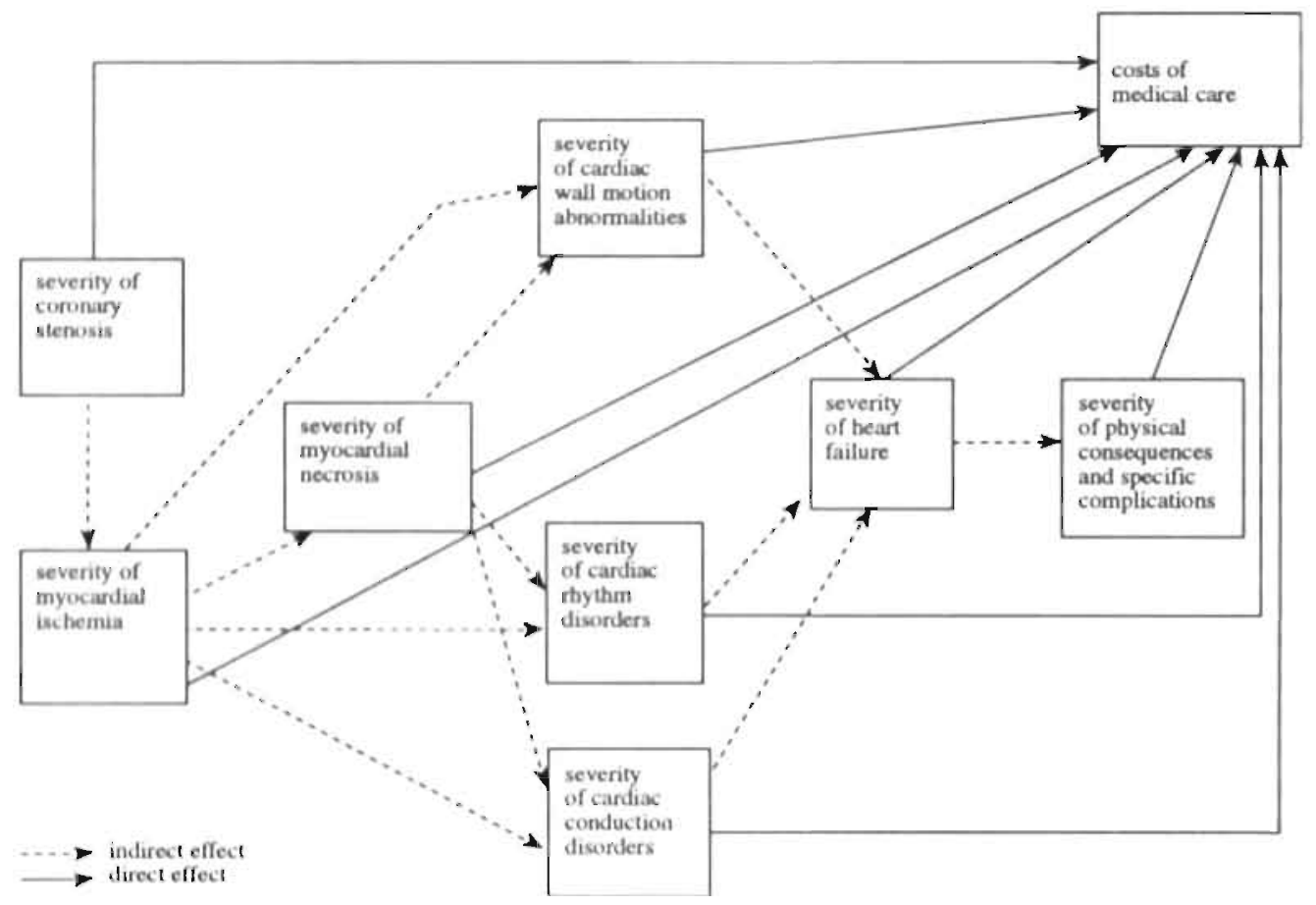

\section{* Controlling for conditional variables.}

Many faccors will play a confounding role in explaining the differences in length of stay or costs of medical care by means of severity of illness. When only the severity indicators are tesied in regression analysis the significant effects may be 'spurious' (not real) and regression coefficients may be overestimated or underestimated due to other factors which have a 'disturbing' effect on the relation between the severity of illness and costs. In order to approach the "real' relations between severivy and the costs as much as possible, these facturs must be included in regression analysis as controlling variables.

As we have seen in $\$ 4.1$. patients may be admitted to and discharged from the cardiology depariment in various ways. The regular hospital admission of AMI patients is the one in which the patient enters the hospital department arriving directly from home. However, not all patients are admitted in this regular way: some patients do get an infaretion while in hospital. Their direct reason for admission might be = for instance - unstable angina pectoris. During the period preceding the infarction these patients will also incur costs for medical care and their overall length of stay will send to be longer than patients who did get an infaretion outside the hospital. In both cases the patient's severity of illness might be similar, yet the costs of staying will be different. So a correction has to be made for this specific type 
of admission (regular or already in hospital measured by DADINTO).

Some other patients are admitted via other hospitals, while they are in various stages of the infarction, but this usually occurs when the acute danger seems to have subsided. This part of the population may have low costs, while at the same time they may have severe complications during their stay. This may also influence the effect of the severity of illness on costs. For instance, some of these patients may have very severe rhythm disorders and may need specialized treatment; it is for this reason that they have been transferred from the local hospital to our university hospital. However, compared to regularly admitted patients with similar rhythm disorders their length of stay and costs will tend to be rather low, because a substantial part of their costs have been made in previous hospital. Furthermore. the moment of entering the hospital during the illness episode may be relevant. Patients entering the hospital in time period $\mathrm{Tl}$ will have different costs in comparison to patients entering the hospital in T3 of the illness episode. Consequently, another correction has to be made for patients coming from other hospitals specified according to the moment of hospital entrance during the illness episode (measured by dummy variables DADEXT1.DADEXT2 and DADEXT3).

Patients may also have a regular or irregular discharge from hospital. The decease of the patient may be seen as a non-regular type of discharge. Patients who die during hospital stay will generally have lower costs compared to non-deceased patients. This study intends to explain costs of medical care. However, in the explanation of mortality by severity of illness a more epidemiologic definition of severity of illness will be necessary, in which clinical or non-clinical risk factors for decease and the actual cause(s) of death during hospital stay will have to be included. Within the economic analysis of costs the problem is: some patients may score very high on the severity indices and at the same time have very low cosis and a very short length of stay, because they have died during stay. For instance patients with specific severe rhythm disorders may have higher chances to die so their length of stay and costs will be very low. On the other hand, non-deceased patients with these same rhythm disorders are expected to have a high length of stay and are expected to have high costs. The 'real' effect of these specific rhythm disorders will be underestimated, if no distinction is made between deceased and non-deceased patients. Another example is patients having a cardiogenic shock. Patients with cardiogenic shock generally have a high risk of dying; their length of stay and costs may he very low despite this severe complication. One might misinterpret this complication as having a negative effect on costs. However, this effect will turn out to be spurious (not substantial or non-real), if a distinction is made betwecn nondeceased patients and deceased patients. Furthermore, patients who die rather soon after the moment of admission will have to be distinguished from patients who die after a prolonged spell of illness in hospital. Thus, the moment (or period) of death of patients will have to be included in each analysis (measured by dummy variables DDEATHO, DDEATH1, DDEATH2 and DDEATH3).

Physicians at the cardiology department will generally act within the boundaries of the medical protocol concerning patients with '(suspected) acute myocardial infarction'. However, a physician does have a certain freedom in treating a patient with $\mathrm{AMI}$ : he/she may be thinking it necessary to keep patients 'under observation', thus prolonging their stay. The reasons for acting this way may be because of specific co-morbidities (like diabetes) or the appearance of complications (like lung embolism, kidney or liver disorders) that are not incorporated in our severity of illness-indicators because of their rarity of the occurrence. Some of these reasons to keep patients in hospital longer may be uniquely related to individual patients with specific illness characteristics. In addition to this physicians may decide to prolong a stay in hospital if there are social indications to keep a patient longer under observation. All these factors are not incorporated in our model and cannot be explained by our severity indicators. The effect of some severity indicators on 
costs, as measured in this study, may be overestimated because of these specific circumstances. For the time being a simple solution is proposed in order to overcome this problem. A 'regular' discharge of AMI patients is expected to be in time period T2. Patients discharged in T3. will be distinguished from regularly discharged patients with a separate dummy variable (DHOME3); these patients may have specific complications or other reasons not captured by our severity indicators, which tend to postpone their discharge.

At the other extreme, there are some patients who leave the hospital relatively soon (within three days) after the moment of admission. This other type of non-regular discharge may also be due to factors not incorporated in our model. Patients may leave the hospital against medical advice or may be transferred to another hospital, because that hospital is nearer to the patient's home residence. They will have a very short length of stay and very low costs due to other reasons than their severity of illness. This coincidence of very low cosis and (moderate) severity of illness might undermine the positive relation between severity of iliness and costs (c.q. length of stay). Therefore again a correction should be made to prevent such an underestimation. Patients discharged in $\mathrm{T} 1$ will be distinguished by a separate dummy variable (DHOMEI).

Using DHOMEI and DHOME3 as typical 'confounders' in regression analysis may encounter some criticism. After all, the introduction of these conditional variables implies that we assume that the 'real' relation between severity of illness during stay and the dependent variables is obscured by two processes. The first process concerns the postponement of discharge by physicians by factors not included in our indices of severity of illness. For instance, patients who have an uncomplicated AMI may still stay very long in hospital for other reasons (clinical or non-clinical). The second process concerns the patients who appear to be (moderate) severe cases, but who are nonetheless discharged very early and usually will be transferred to other hospitals. The decision to use this solution in the multivariate analysis is preferred to that of not correcting for these situations of bias, which in our view is detrimental to finding the 'real' relationship between costs of care and severity of illness. In the following paragraphs we will also provide the reader with altemative models of analysis, in which we did not include these factors, so that a comparison of results will be possible.

Another 'non-regular' discharge type from the cardiology department is: transferral to another medical department in the same hospital. When patients are transferred to other medical departments of the University Hospital, they will generally have specific complications which need to be treated by physicians from other medical specialties. In this special case, the overall length of stay will tend to be much longer, but it will not only be caused by the severity of the cardiac problems. Thus, the effect of the severity indieators measured in this study may be overestimated. Furthermore, it is clear that when patients are transferred during an early phase of the stay they will have lower costs compared to patients transferred later on. Therefore, the moment of transfer needs 10 be incorporated into the cost analysis as well (measured by dummy variables DTRANS1, DTRANS2 and DTRANS3). Results from the analysis may thus be interpreted as the effect of the severity of illness on the length of stay and costs of care within the cardiology department only.

In cost analysis of medical care even the length of stay in days may play a confounding role. Patients with severe conditions may have apparently high costs, but these higher costs will be strongly related to their long length of their stay. So, specific care costs uniquely due to the severity of illness and costs due the length of stay need to be separated. In other words: there are costs directly caused by the severity of illness and there are costs simply related to length of stay. Corrections should be made to find the relation between costs and severity of illness by restricting it to what is uniquely and directly due to the severity of illness. 
Patients" background characteristics, such as age, sex, previous infarction or angina pectoris, are assumed to be related to the length of stay and the costs of medical care, as well as the severity of illness. For instance, the illness condition of older patients will usually be more severe and because of this these patients will have higher costs and a longer hospital stay. Thus. to determine the impact of severity of illness on the costs of medical care one should again statistically correct for old age.

All factors mentioned above may be strongly related to the severity of illness, the length of stay and the costs of medical care. To measure the effect of severity of illness on length of stay or costs of medical care, a multivariate analysis must be performed using the severity of illness indices as 'independent' variables together with all conditional factors related to severity of illness as well as to the dependent costs variables (c.q. length of stay). Only in such a model can the 'real' influence of severity of illness on the cost of medical care (c.q. length of stay) be determined. In the subsequent multivariate analysis a patient's hospital admission and discharge status together with his or her length of hospital stay and background characteristics will be regarded as conditional variables in the explanation of costs by severity of illness. The conditional variables can not be seen as an explanation of costs (they are only correlated to costs), but must principally be regarded as correction factors.

The relationships and effects between all variables are represented in a path-analytic model (diagram 7.2), which forms an extension of diagram 7.1 by incorporating the model in diagram 3.3. The severity of illness indicators can be operationalized into the many (dynamic) severity of iliness categories as described in $\$ 6$.

Diagram 7.2 Model explaining length of stay and costs of medical care by indicators representing severity of illness, given some conditional variables (see text).
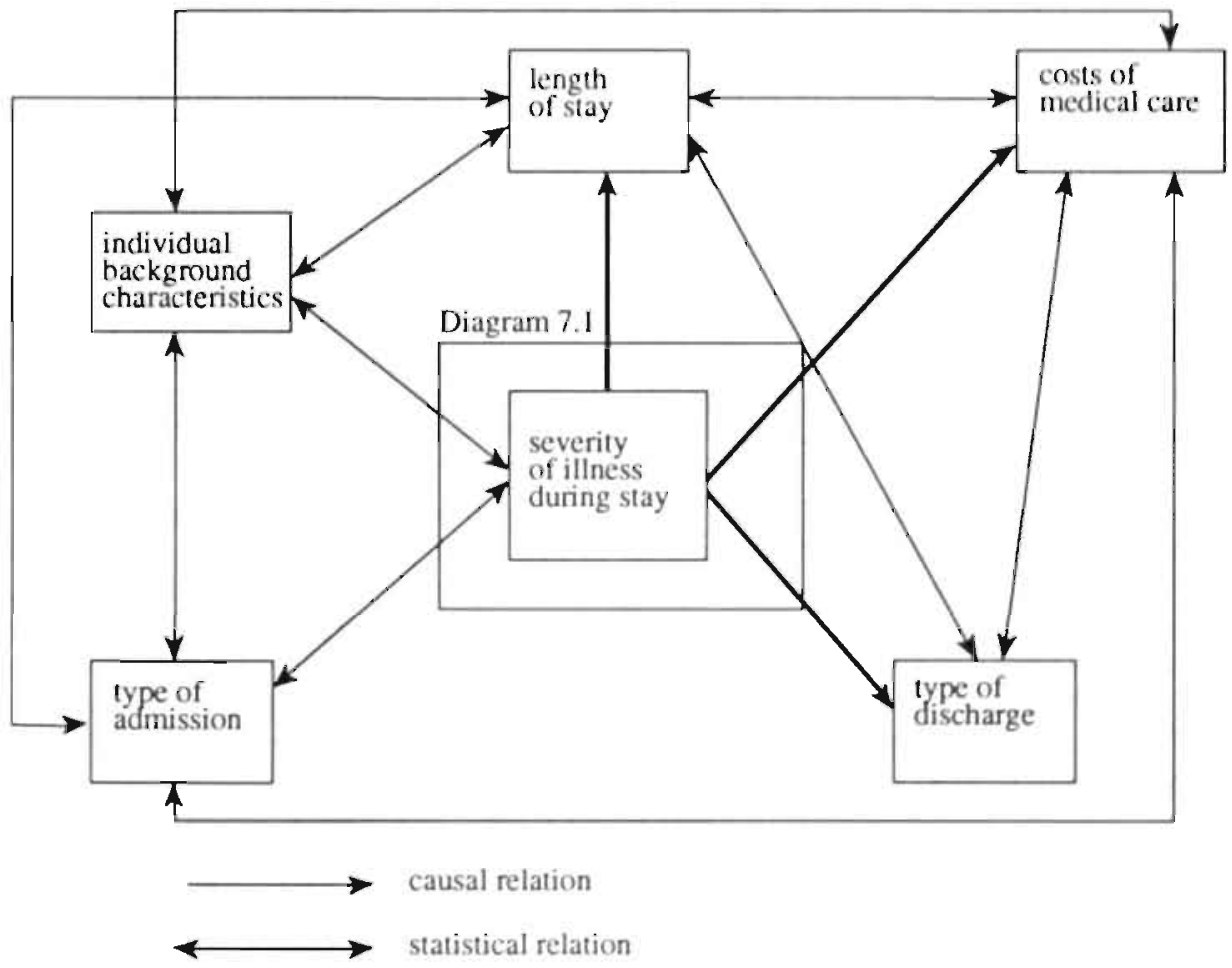


\subsection{Period specific cost analysis.}

In this paragraph results are presented regarding the multivariate analysis of:

* Costs of thrombolytic therapy in T0 $(7: 2,1)$,

* Costs of (acute) heart catheterization in T0 (7.2.2), and

* Costs of (acute) PTCA in T0 (7.2.3).

In these analyses time-specific severity indicators will be used to explain cost variations; within period T-0.

\subsubsection{Explanation of costs of thrombolytic therapy.}

Thrombolytic therapy is one of the most important interventions when patients suffer from acute myocardial infarction. To be effective it has to be administered within the first hours. that symptoms appear. Thus, the cost differences in thrombolytic therapy can only be investigated for the first six. critical hours of hospital admission (period T0). Several sources may provide an explanation for cost differences: (1) the individual medical history and personal characteristics of the patient; (2) earlier cardiovascular (or other) deficiencies, and pre-admission diagnostics and treatment of cardiologic nature; (3) the severity of illness as reported by the patient, for example, concerning the duration of ischemic pain; (4) the severity of illness as registered by the admission. ECG of the patient; this includes the determination of the severity of myocardial ischemia, myocardial necrosis, cardiac rhythm disorders and cardiac conduction disorders.

A pre-analysis had to be performed in order to remove all non-stochastic (fixed) relations in the explanation of the costs of thrombolytic therapy. Non-stochastic relations will exist. when there are (protocol-based) contra-indications for treatment. All patients at the University Hospital Maastricht over 75 years of age, in coma or with more than six hours of typically ischemic pain are contra-indicated for thrombolytic therapy during the period of the investigation. Patients with these characteristics are excluded from further analysis $(\mathrm{n}=128) .28 .5 \%$ of the variance in costs of thrombolytic therapy can be 'explained' using these three contra-indications for treatment (AGE: $11.2 \%$, COMA: $2.4 \%$. PAIN more than 6 hours: $11.0 \%$, AGE and COMA: $6 \%$, AGE and PAIN more than 6 hours: $3.3 \%$ ). Furthermore, all patients $(n=59)$ enrolled in the local PRIMI protocol did receive thrombolytic therapy for the mere reason they participated. Because the dependent variable is also constant for these patients. no statistical analysis could be performed for this group of patients 600 . From a statistical point of view the participation in this clinical trial alone 'explains' $16.6 \%$ of the variance in costs. In total 187 patients are removed from further regression analysis.

The remaining sample population $(n=277)$ is used for explaining costs of thrombolytic therapy seen as a stochastic dependent variable. In this population, presence or absence of ischemic pain was recorded for 184 patients; 93 patients had missing values on this variable. In order to include all patients in the regression analysis two dummy variables haven been constructed (DPAIN1: patients with no ischemic pain contrasted with patients with ischemic. pain, and DPAIN2: patients with no ischemic pain contrasted with patients with missing values). Four patients were removed as outliers because of extremely high costs. Some descriptive statistics of this subpopulation are given in table 7.3. 
Table 7.3 Descriptive statistics of the patient population used to explain costs of thrombolytic therapy (Listwise deletion of missing cases, $n=254$ ).

\begin{tabular}{llrrr}
\hline Variable & Description & Mean & Std.dev & Range \\
& & & & \\
CTHROMO & costs of thrombolytic therapy in TO & 169.02 & 270.74 & $0-1115.10$ \\
THROMO & thrombolytic therapy in TO & .29 & .46 & $0-1$ \\
ISEXTO & ischemia extent in TO & 1.48 & .68 & $0-3$ \\
ISLOCO & ischemia location in TO & 1.28 & .54 & $0-2$ \\
NECEXTO & necrosis extent in TO & .90 & .51 & $0-2$ \\
NECLOCO & necrosis location in TO & 1.06 & .65 & $0-2$ \\
DISPAINI & not having ischemic pain & .65 & .48 & $0-1$ \\
DISPAIN2 & no ischemic pain/having missing values & .30 & .46 & $0-1$ \\
AGE & patient's age & 59.77 & 9.35 & 31.74 \\
SEX & male(O)Vfemale(1) & .26 & .44 & $0-1$ \\
\hline
\end{tabular}

About $29 \%$ of the remaining patients receive thrombolytic therany and the mean costs per patient amount to 169.02 guilders (std.dev. 270.74), Variables showing significant correlations with the costs of thrombolytic therapy are presented in appendix 17.

The extent and the location of myocardial ischemia are positively correlated with costs of thrombolytic therapy (ISEXTO: $r=, 24 ; p<001$, ISLOC0; $r=, 11 ; p<.05$ ). Similar results are found for the extent and the location of myocardial necrosis (NECEXT0:r=.20;p<.01, NECLOCO: $r=19 ; p<.001)$. The dummy variable representing the contrast between no ischemic pain and typically ischemic pain is also highly positively correlated to costs of thrombolytic therapy (DISPAIN1: $\mathrm{r}=.31 ; \mathrm{p}<.001$ ). The contrast between no ischemic pain and missing values is highly negatively related (DISPAIN2:r=-.26;p<.(00)). All other variables (like severity of cardiac rhythm disorders, conduction disorders and background characteristics) show no significant correlations with the dependent variable.

All significantly correlated variables are used as independent variables in a multiple regression analysis. In the eventual regression analysis only three variables have significant parameters: the severity of necrosis location, the severity of the extent of ischemia and the presence of ischemic pain (table 7.4 ). $17 \%$ of the variance in costs of thrombolytic therapy can be explained by these indicators.

Table 7.4 Results from regression analysis: the dependent variable is costs of thrombolytic therapy in T0 (Listwise deletion of missing cases, $n=254$ ).

\begin{tabular}{|c|c|c|c|c|}
\hline Independen Variables & $B$ & $S E B$ & Beta & Sig $T$ \\
\hline ISEXTO & 68.44 & 23.94 & 17 & .005 \\
\hline NECLOCO & 71.64 & 24,82 & 17 & .004 \\
\hline DISPAINOI & 195.94 & 67.17 & .35 & .004 \\
\hline DISPAINO2 & 31.30 & 70.56 & .05 & NS \\
\hline CONSTANT & -144.18 & 75.82 & & NS \\
\hline \multicolumn{5}{|c|}{$\begin{array}{l}\text { Explained Variance }\left(R^{2}\right): . I 7 \\
B=\text { unstandardized (partial) regression coefficient: } S E B=\text { standard error belonging io } B ; B E T A= \\
\text { standardized (partial) regression coefficient; } S I G T=\text { significance of } B \text { or } B E T A \text { according to the } \\
T \text {-distribution (I degree of freedom) }\end{array}$} \\
\hline
\end{tabular}




\section{Discussion.}

It can be concluded that for patients with (suspected) AMI, who do not have contraindications and who are not involved in a randomized clinical trial, the probability of getting thrombolytic therapy will increase if patients show signs of anteroseptally located necrosis in the ECG. This probability will also increase, if patients show extensive left ventricular wall ischemia in ECG, and if patients indicate that they are having typical ischemic pain. This last effect turns out to be twice as important as the others. The relative importance of each effect on the costs of thrombolytic therapy (given by the beta parameters) is presented in diagram 7.7. Cardiologists did confirm that the criteria found empirically are also specified in the thrombolytic protocol which was used at the cardiology department during 1987-1988.

\subsubsection{Explanation of costs due to (acute) heart catheterization.}

For the patient population at hand the greater part of the costs due to heart catheterization are incurred during the acute phase of the infarction in $\mathrm{TO}$ (see appendix 5). Differences in costs in this time period will be analysed. Although heart catheterization may have been performed in $\mathrm{T} 1$ and sometimes in periods T2 and T3, these costs will not be discussed here.

Non-stochastic relations as a result of $100 \%$ indications for acute heart catheterization haven been eliminated from the eventual regression analysis. Enrollment in the PRIMIprotocol was an indication for applying a catheterization. Circumstances with contraindications for catheterization were not present. In total 59 patients have been excluded from regression analysis because of their enrollment in the PRIMI protocol (variance explained amounts to $13.1 \%$ ). In addition, 3 patients have been excluded from further analysis because of extremely high catheterization $\cos t s(m=1095.79$, std.dev. $=632.850)$. The remaining sample population is used in explaining costs for acute catheterization. Some descriptive statistics of this subpopulation are presented in table 7.5 . In this sample population $26 \%$ of the patients is given a catheterization and the mean costs per patient amount to 231.18 guilders $($ std.dev. $=400.96)$.

Table 7.5 Descriptive statisties of the patient population used to explain costs of acute heart catheterization during period T0 (Listwise deletion of missing cases, $n=378$ ).

\begin{tabular}{llrrr} 
Variable & Description & Mean & Std.dev & Range \\
CCATO & costs of heart catheterizations in TO & 231.18 & 400.96 & $0-1624.13$ \\
CATO & number of catheterizations in TO & .26 & .44 & $0-1$ \\
ISEXTO & ischemia extent in TO & 1.46 & .71 & $0-3$ \\
ISLOCO & ischemia location in TO & 1.26 & .56 & $0-2$ \\
NECEXTO & necrosis extent in TO & .90 & .53 & $0-3$ \\
NECLOCO & necrosis location in TO & 1.07 & .67 & $0-2$ \\
THROMO & thrombolyric therapy in TO & .21 & .41 & $0-1$ \\
FTHROM & former thrombolytic therapy & .03 & .18 & $0-1$ \\
AGE & patient's age & 63.61 & 11.96 & $31-91$ \\
SEX & male(O)yfemale(1) & .32 & .47 & $0-1$ \\
DADINTO & internal admission & .03 & .18 & $0-1$ \\
\hline
\end{tabular}


Variables showing significant correlations with the costs of acute heart catheterization are presented in appendix 18 . Both indices expressing the severity of myocardial ischemia measured by ECG on admission show significant correlations with the costs of acute heart catheterization (ISEXCT0: $r=.19 ; p<.001$ and ISLOCO: $r=.14 ; p<.01$ ). Similar results are found for the variables expressing the severity of myocardial necrosis measured by ECG (NECEXT0:r=.12;p<.01 and NECLOC0:r=.13;p<.01). A high correlation is found between catheterization costs and receiving or not receiving thrombolytic therapy during current admission (THROM:r=.50;p<.001). Earlier thrombolytic therapy, given before the current hospital admission, appears to be positively correlated with costs of acute catheterization as well (FTROM: $r=.12 ; p<.05)$. The patient's age and $\mathrm{sex}$ appear to be negatively correlated with the costs (AGE:-.20;p<.001 and SEX:r=-.20;p<.001). Furthermore, having an infarction while in hospital is moderately correlated with costs of acute catheterization (DADINT0: $r=.27 ; p<.001$ ).

Five independent variables do show significant parameters in the eventual regression analysis: former and current administration of thrombolytic drugs, the extent of ischemia, sex and type of admission (table 7.6). It may be concluded that the probability of getting a catheterization during the first six. hours of hospital stay for patients with (suspected) AMI will increase, if patients receive(d) thrombolytic therapy during the current or a previous hospital stay. Moreover, patients with ST-changes corresponding with many wall segments are likely to incur costs due to catheterization. Female patients have a significantly lower chance of receiving an acute catheterization. Lastly, patients who have an infarction while in hospital (DADINT0) are more likely to receive a catheterization during the acute phase of the infarction than other patients. As can be seen from the results of the regression analysis (table 7.6), $37 \%$ of the variance in costs of acute catheterization is explained by means of these indicators. All other factors offer no significant, additional explanation for the variance in costs of acute heart catheterization.

Table 7.6 Results from regression analysis; the dependent variable is costs of acute heart catheterization in T0 (Listwise deletion of missing cases, $n=378$ ).

$\begin{array}{lrrrr}\text { Independent Vuriables } & B & \text { SE B } & \text { Beta } & \text { Sig T } \\ \text { ISEXTO } & 66.35 & 23.60 & .12 & .005 \\ \text { THROMO } & 465.85 & 41.21 & .48 & .000 \\ \text { FTHROMO } & 340.19 & 94.41 & .15 & .000 \\ \text { SEX } & -92.18 & 35.86 & -11 & .011 \\ \text { DADINTO } & 609.36 & 93.85 & .27 & .000 \\ \text { CONSTANT } & 126.82 & 63.77 & & .048\end{array}$

Explained Variable $\left(R^{2}\right): .37$

$B=$ unstandardized (partial) regression coefficient; $S E B=$ standard error belonging to $B ; B E T A=$ standardized (partial) regression coefficient: SIG $T=$ significance of $B$ or BETA according to the T:distriburion (I degree of freedom) 


\section{Discussion.}

Costs of acute heart catheterization for patients with AMI are only partly explained by the patient's severity of illness; ischemia (measured by ECG) extensive over more wall segments has a moderate effect on the costs of acute heart catheterization. Medical treatment, i.e. thrombolytic therapy, has an effect on costs of acute heart catheterization, which is four times larger than the effect of the extent of ischemia. If thrombolytic therapy is given (particulariy during the current hospital stay) this certainly tends to increase the costs of acute heart catheterization. Cardiologists did confirme this conclusion. In 1987-1988 by protocol it was defined that to ascertain the clinical efficacy of thrombolytic therapy a catheterization was needed. In addition, female patients are less likely to receive an acute heart catheterization. There is no explanation for this finding. However, in other studies this apparently 'strange' relation was also found (Steingard 1991, Ayanian 1991). Lastly, patients who have an infarction while in hospital are more likely to receive a heart catheterization during the first hours that symptoms appear. The relative importance of these variables on the costs of acute catheterization (beta parameters) is presented in diagram 7.7 together with the analysis results for the costs of thrombolytic therapy (see $\$ 7.2 .1$ ).

Diagram 7.7 Path-analytic model combining the explanation of costs of thrombolytic therapy and costs of heart catheterization in T0 by severity of illness. Beta parameters are shown (Pairwise deletion of missing cases, $254 \leqslant n \leqslant 378$ ).

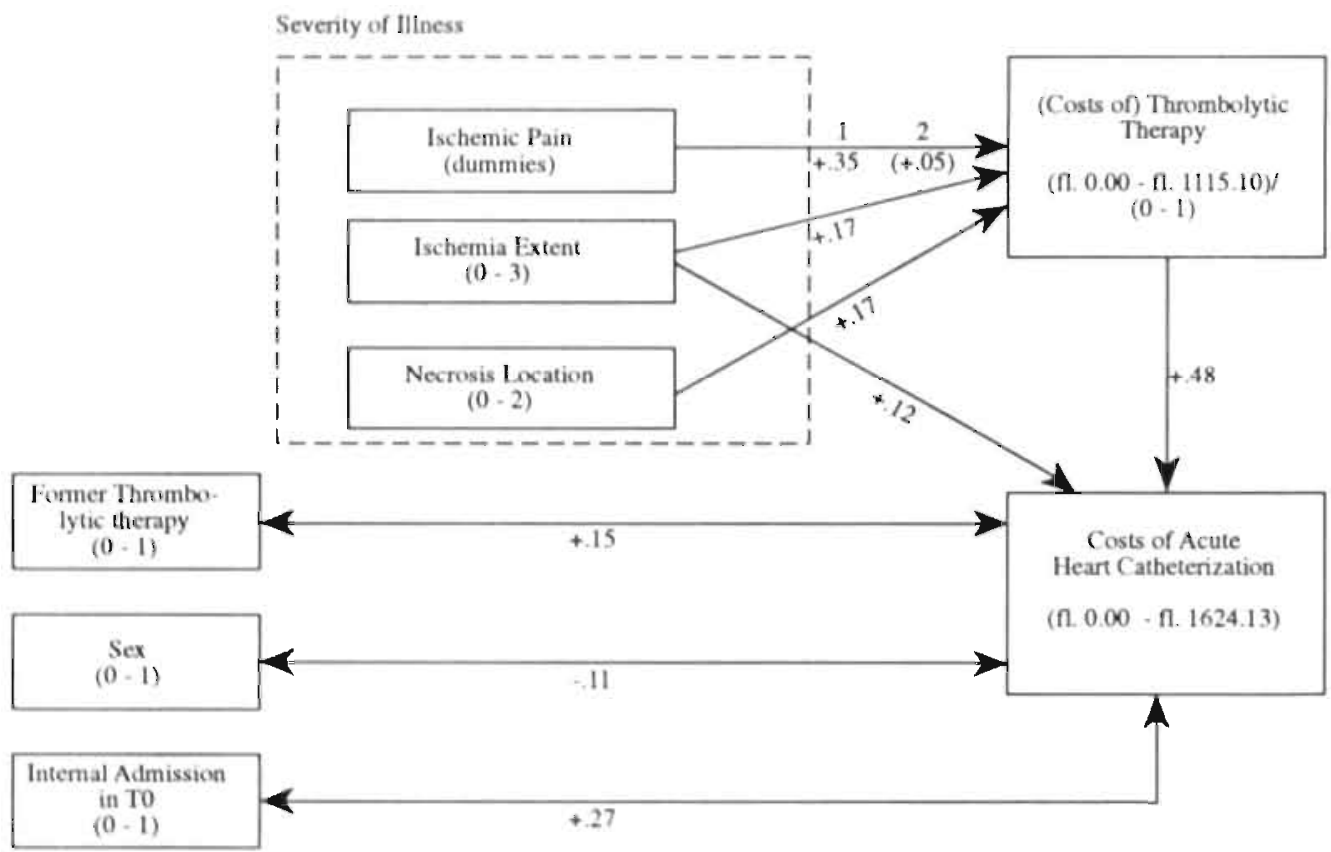

Explained Variance (R:2): 17 (costs of thrombolytic therapy)

37 (costs of acute heart catheterization) 


\subsubsection{Explanation of costs due to (acute) PTCA.}

Costs due to PTCA are incurred generally during the first six hours of hospital stay (sce appendix. 12). The decision to perform an acute PTCA is, amongst other factors, based on the insufficiency of coronary flow and the severity of coronary stenosis before PTCA and after thrombolytic therapy. Another important criterion in the decision making process may concern the severity of illness as indicated by the ECG. In addition, it is assumed that patients who do receive thrombolytic therapy but whose coronary angiogram still indicates a severe coronary stenosis, will have a higher chance of receiving a PTCA. (interaction effect).

Of the 159 catheterized patients in T0, 82 patients $(51.9 \%)$ receive only thrombolytic therapy and no PTCA, 18 patients $(11.4 \%)$ receive only' PTCA and no thrombolytic therapy, 32 patients: $(20.3 \%)$ receive both and 26 patients $(16.5 \%)$ do not get either of these treatments. Almost one third of the catheterized patients undergoes a PTCA. Costs of PTCA are constant for each patient. (fI. 3677.24). Therefore, in the regression analysis, the cost of PTCA can be substituted by a dichotomous variable 'PTCA: yes or no'. Some deseriptive statistics of the subpopulation used to explain costs of PTCA in TO are presented in table 7.8 .

Table 7.8 Descriptive statistics of patient populations used to explain costs of PTCA in TO.

\begin{tabular}{|c|c|c|c|c|c|c|c|c|c|c|c|}
\hline \multirow[b]{3}{*}{ Vwewhir } & \multirow[b]{3}{*}{ Descriptice } & \multirow[b]{3}{*}{ kangr } & \multirow{2}{*}{\multicolumn{3}{|c|}{ all calterusul AMI petimes }} & \multirow{2}{*}{\multicolumn{3}{|c|}{ 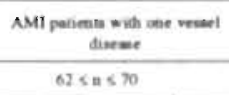 }} & \multirow{2}{*}{\multicolumn{3}{|c|}{ 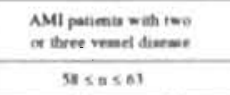 }} \\
\hline & & & & & & & & & & & \\
\hline & & & $m$ & ond der & a & m & ned are & $n$ & in & ost ine & $m^{\prime \prime \prime}$ \\
\hline CPTCAO & Couts of PTCA in To & 0.3672 .24 & 1217.79 & 1736.28 & 154 & 1172.45 & 1726.25 & $\infty$ & 145822 & 181459 & 4 \\
\hline NHTANO & Number of PTCA in T0 & $0-1$ & 3 & 47 & 152 & 32 & 47 & 60 & 4) & 40 & 57 \\
\hline THKOM & Thrombolytic therapy in $\mathrm{T} 0$ & 0.1 & 72 & 45 & 158 & nt & 39 & 70 & 3 & 45 & 62 \\
\hline STENINO & Stenosis intensiny in To & .93 .173 & .01 & 53 & 129 & .35 & 25 & 70 & 17 & 47 & 61 \\
\hline STENEXO & Stencosit extent in $\mathrm{TO}$ & 0.3 & 100 & $\pi$ & 129 & 97 & 17 & 70 & 233 & $\Delta$ & 63 \\
\hline STENLOOX & Stenosula bcation in $\mathrm{TO}_{0}$ & 0.19 & 8.67 & 4.42 & 129 & 540 & 2.10 & $x_{0}$ & 1237 & 313 & 63 \\
\hline NECEXO & Necrovas enent in To & a-3 & 1.07 & a & 150 & 107 & 90 & $\pi$ & 100 & $\omega$ & 62 \\
\hline SECTOO & Necross location in To & 0.2 & 124 & 50 & 150 & 129 & 52 & 70 & 113 & 53 & 62 \\
\hline ISCEXO & Lechemis event in TO & a.3 & 108 & $\pi$ & 159 & 160 & 63 & 70 & 105 & 73 & 62 \\
\hline 15quini & Lachemis bocation in TO & 0.2 & 135 & 36 & 159 & 130 & 55 & 70 & 126 & 57 & 62 \\
\hline INTER 10 & Throm - Simin in T0 & .93 .1 .34 & .05 & 41 & 129 & .29 & 26 & 70 & 20 & wo & 62 \\
\hline INTER:20 & Throm - Sienes in To & a. & 1.21 & क & 129 & so & a) & to & 179 & 114 & 62 \\
\hline ACE & Patienti Asge & y1:-91 & 99.40 & 9.67 & 194 & $36 \pi$ & 9.63 & 70 & 9913 & $\mathrm{~s} 3 \mathrm{~s}$ & 63 \\
\hline SEX & Male (O) Femaic (1) & 0.1 & 21 & .4 & 159 & 20 & 40 & 70 & 24 & 43 & 63 \\
\hline FCOR & Former coransy unoufficiency & 0.1 & 13 & 31 & $15 \mathrm{n}$ & as & 20 & 70 & 25 & 43 & 6) \\
\hline FAMI & Former AMII & $a_{1}$ & 20 & $\$ 0$ & 15s: & $\infty 6$ & 23 & \% & 36 & 4 & 61 \\
\hline EHYE & Former typenenaion & 0.4 & 23 & 42 & 158 & 29 & $\omega$ & 70 & 17 & 38 & 61 \\
\hline
\end{tabular}

(1) Differences in number of pursents occurs becouse of mining cats 
As can be seen from the correlations between the dependent and independent variables (appendix 19) there is a positive correlation between the coronary intensity score and PTCA $(r=.24, p<.001)$. Chances to get PTCA seem to decrease somewhat when patients get thrombolytic therapy $(r=-.21, p<.001)$. A first regression analysis shows a very low explained variance in the costs of PTCA. Subsequently, specific interaction effects have been tested. The first interaction effect concerns getting thrombolytic therapy and the intensity of coronary stenosis on the costs of PTCA (INTER1). The second interaction effect concerns getting thrombolytic therapy and the extent of stenosis (INTER2). The reason why this last term was calculated may not be very obvious at first, since the first interaction term has a reasonably high correlation with the dependent variable $(r=.31 ; p<.001)$ and the other term not at all $(r=-.03 ; p=.30)$. However, when both terms are used in multiple regression analysis, it turns out that the first interaction effect is not significant anymore, when the second interaction effect is included. This rather puzzling result may be explained by a confounding effect of the extent of stenosis (STENEX0). After several analyses the crucial variable in the analysis of performance of PTCA turns out to be the extent of stenosis (STENEX0), Interpretation of results will improve considerably if patients with one vessel discase $(n=70)$ are separated from patients with two or three vessel disease $(n=59)$. Descriptive statistics of these two subgroups of patients are also presented in table 7.8 . In the corrclation matrices for each subpopulation (tables 7.9 and 7.10) we see that for patients with one vessel disease there is a high negative correlation between costs of PTCA and thrombolytic therapy $(\mathrm{r}=-.39 ; \mathrm{p}<.001)$ and a high positive correlation between the intensity of coronary stenosis and costs of PTCA $(r=.35 ; \mathrm{p}<.001)$. For patients with two or three vessel discase only the intensity of coronary stenosis shows a significant correlation with costs of PTCA $(r=.22 ; p<.01)$.

Tuble 7.9 Correlation matrix of severity of illness, costs of thrombolytic therapy and costs of PTCA in To for patients with one vessel disease (List wise deletion of missing cases, $n=69$ ).

CPTCAO THROMO STENINO STENLOO NECLOO

$\begin{array}{lllll}\text { CPTCAO } & 1.00 & & & \\ \text { THROMO } & .39^{*} & 1.00 & & \\ \text { STENINO } & .35 * & .02 & 1.00 & \\ \text { STENLOO } & .12^{*} & .14^{*} & .05 & 1.00 \\ \text { NECLOO } & .23^{*} & .17^{*} & .07 & .42 * \\ { }^{*} P<.05 & & & & \end{array}$

Table 7.10 Correlation matrix of severity of illness, costs of thrombolytic therapy and costs of PTCA in T0 for patients with two or three vessel disease ( $L$ ist wise deletion of missing cases, $n$ $=58$ ).

\begin{tabular}{lccccc}
\hline & CPTCAO & THROMO & STENINO & STENLOO & NECLOO \\
CPTCAO & 1.00 & & & & \\
THROMO & -.02 & 1.00 & & & \\
STENINO & $.22 *$ & $-.33^{*}$ & 1.00 & & \\
STENLOO & $.16^{*}$ & .05 & $.41^{*}$ & 1.00 & 1.00 \\
NECLOO & $-14^{*}$ & $-.26^{*}$ & $-10^{*}$ & .05 & \\
$* p<.05$ & & & & & \\
\hline
\end{tabular}


Regression analysis using the data of patients with one vessel disease shows three significan! explaining factors: thrombolytic therapy, the intensity of coronary stenosis and the location of necrosis. In total, $35 \%$ of the variance in costs of PTCA for this subgroup of patients is explained by these factors (table 7.11).

For the second group of patients with multiple vessel disease (two or three vessel disease) the costs of PTCA can only be explained by the intensity of coronary stenosis (Beta: 22 ). For this subgroup only $5 \%$ of the variance in costs of PTCA could be explained.

Table 7.11 Results of regression analysis; the dependent variable is costs of PTCA in T0 for patients with one vessel disease (Listwise deletion of missing cases, $n=69$ ).

$\begin{array}{lrrrr}\text { Independent Variables } & B & \text { SE B } & \text { Befa } & \text { Sig } T \\ \text { STENINO } & & & & .0018 \\ \text { NECLOCO } & 2276.77 & 700.66 & .33 & .0074 \\ \text { THROMO } & 783.65 & 283.58 & .48 & .0001 \\ \text { CONSTANT } & -1876.74 & 445.85 & .43 & .0000\end{array}$

Explained Variance $\left(R^{2}\right):, 35$

$B=$ unstandardized (partial) regression coefficiem; $S E B=$ standard error belonging to $B ; B E T A=$ standardized (partial) regression coefficient; SIG $T=$ significance of $B$ or BETA according to the T-distribution (1 degree of freedom)

\section{Discussion.}

In spite of the relative failure to explain costs of acute PTCA by our severity indices in patients with two and three vessel disease, these costs can with a certain amount of success be explained in patients with one vessel disease. Patients with one vessel disease have a lower chance of getling a PTCA, if they receive thrombolytic therapy. However, chances of receiving a PTCA increase, if they have a total coronary occlusion. If these paticnts have anteroseptal necrosis, there is an additional chance of receiving a PTCA. The relative effect of these variables on the cosis of PTCA (beta parameters) is presented in diagram 7.12. Patients with two or three vessel disease also have higher chances of receiving PTCA, if they have total coronary occlusion; however the explained variance is rather low. For this group of patients thrombolytic therapy turns out to have no relation to the costs of PTCA. To interpret these results some cautionary remarks must be made regarding our model of explanation; the analysis is hampered in several ways because it appears that next to the intensity of coronary stenosis the decision to perform PTCA is also dependent on the antegrade coronary flow and the adequacy of collateral blood flow. It is not possible in this stage of the research to quantify the way cardiologists judge the (in)sufficiency of this collateral blood flow. In addition to this measurement probiem, there are many contraindications for performing PTCA which should be taken into account in data analysis. Some of these contra-indications are almost uniquely related to individual patients. In summary, our model of explanation is - in hindsight - not satisfactorily specified for all variables, that may be related to both the costs of PTCA and the characterisitcs of coronary stenosis. 
Diagram 7.12 Path analytic model used to explain costs of PTCA in T0 by severity of illness in patients with one vessel disease. Beta parameters are shown (Listwise deletion of missing cases, $n=69$ ).

Thrombolytic

therapy

Severity of illness

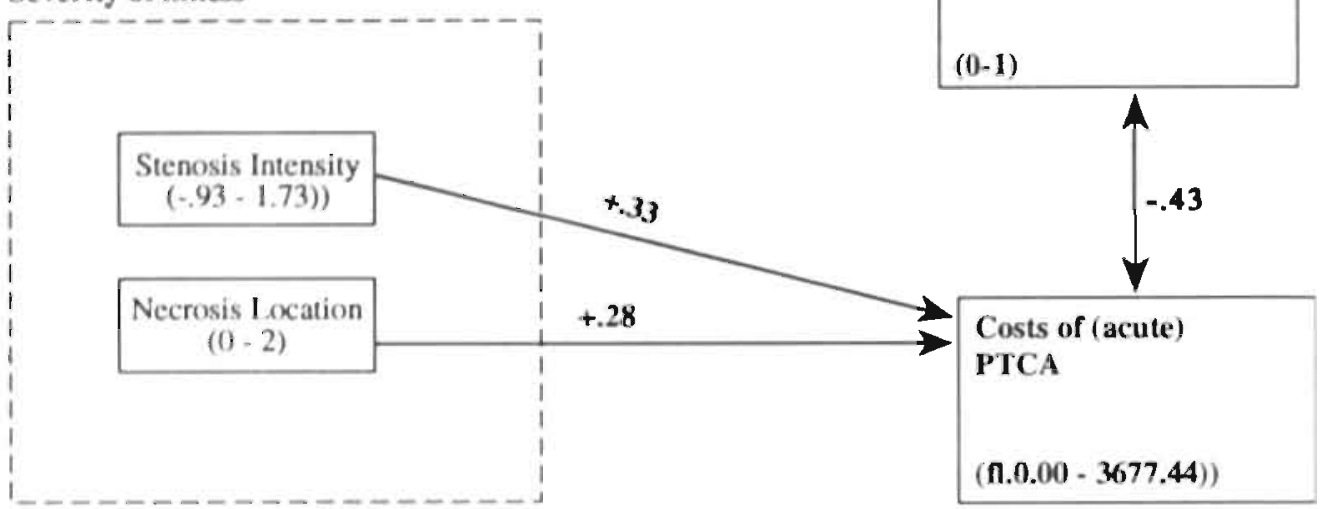

Explained Variance $\left(\mathbf{R}^{2}\right): .35$ 


\subsection{Analysis of length of stay and costs in medical care during total hospital stay.}

In this paragraph results of the multivariate analysis are presented for:

* Length of hospital stay (7.3.1);

* Costs of coronary care unit (7.3.2);

* Other costs of medical care (drugs, laboratory services, electrocardiogram, echocardiogram and exercise tests) (7.3.3).

In this analysis (dynamic) severity categories deduced from the severity indicators as shown in $\$ 6$ will be used to explain length of stay or cost variations. Furthermore all decisions and rules on methodology and/or statistics will be applied that are described in $\$ 7.1$.

\subsubsection{Explanation of length of stay.}

The overall length of stay is defined as the total number of days a patient is in hospital irrespective of the medical speciality. This means that the number of days could have been spent at other wards than those of the Department of Cardiology. For the total patient population of 464 patients the mean length of stay amounts to 11.45 days (st.dev. $=9.60)$ ). The duration of stay varies between 1 and 112 days. Seven patients were removed form analysis because of their extremely long stay $(m=66.29$, std.dev. $=29.32)$. In the remaining population, now amounting to 457 patients, the mean length of stay comes down to 10.61 days (st.dev. $=5.95$ ). Duration of stay now varies between 1 and 42 days.

Significant correlations between (dynamic) severity categories, background characteristics and length of stay are presented in appendix 20 . For nominal predictors special tables will be presented showing the relation with length of stay by means and standard deviations in each category.

\section{* Severity of coronary stenosis.}

Variance in length of stay explained by the dummy variables representing the categorics of intensity of stenosis, the extent of stenosis and location of stenosis (sec \& 6.2.1) turns out to be rather low ( $\mathrm{Eta}^{2}$ is resp. 0.16,0.11 and 0.13 ). If the three sets of dummy variables are seprarately used as predictors in the regression analysis, none in the sets of variables is statistically significant. If patients have a catheterization their length of stay seems to increase somewhat $(r=.09 ; \mathrm{p}<.05)$. This low positive correlation turns out to be nonsignificant in the eventual regression analysis.

It may concluded that the severity of coronary stenosis has no substantial effect on the length of stay of AMI patients.

\section{* Severity of myocardial ischemia.}

The severity of myocardial ischemia measured by the ECG at rest ( $\operatorname{tec} \$ 6.2 .2$. i.) is significantly correlated with length of stay. The correlation matrix (appendix 20) shows that patients with anteroseptal myocardial ischemia (ISLOC) have a significantly higher chance of staying in hospital longer in comparison to patients with non-anteroseptal ischemia $(r=27 ; p<.001)$. In the eventual regression analysis this effect remains significant (table 7.19). The extent of myocardial ischemia (ISEXT) has a lower but nonetheless significant correlation with the length of stay $(r=.11 ; p<.05)$. Patients with extensive myocardial ischemia seem to have a significantly higher chance of staying in hospital longer. In the eventual regression analysis this turns out to be a non-significant parameter. Furthermore, when patients do have extensive myocardial ischemia chances are high that it will be located 
in the anteroseptal side of the left ventricle $(r=.39 ; \mathrm{p}<.001)$.

The dummy variables comprising the severity of myocardial ischemia measured by the exercise test according to the Bruce protocol or measured by the thallium test (see \$6.2.2.2.) show no significant correlations with the length of stay. This is not surprising: normally these tests are performed when the moment of discharge has already been planned by the treating physician. These tests are usually made: one or two days before the patient is planned to leave the hospital. The results of these tests may be relevant for further treatment decisions in an outpatient setting, but the tests have no large consequences for the total duration of the current hospital stay.

It may be concluded that the location of myocardial ischemia has a substantial effect on the length of stay; anteroseptally located myocardial ischemia will contribute to an increase in the number of hospital days.

\section{* Severity of myocardial necrosis.}

According to appendix 20 the location of the severity of myocardial necrosis determined by means of ECG (see \$ 6.2.3.1.) Iurns out to be positively correlated with the length of stay (NECLOC:r=18:p<.001). it seems that patients with anteroseptal necrosis tend to stay in hospital longer than other patients. However, in the eventual regression analysis the socalled effect turns out to be non-significant. Furthermore, when patients have anteroseptal necrosis, chances are high that they will also have anteroseptal ischemia $(r=.63 ; p<.001)$. However, in the explanation of length of stay, anteroseptal ischemia appears to be a more important explaining factor than anteroseptal necrosis. The extent of myocardial necrosis (NECEXT) does not turn out to have a significant correlation with the length of stay at all $(\mathrm{r}=.05 ; \mathrm{p}=.15)$.

Severity of myocardial necrosis, measured by means of typical enzyme release (see 6.2 .3 .2 .), appears 0 have a significant relation with the length of stay. An increase in length of stay is generally moderately related with a patient's increase in enzyme release. As can be secn in table 7.13, oneway variance explained by the CPK severity categories amounts to 06 (p<.001). Only dummy variable DCPK5 (CPK release $>60000$ ) has a significant (positive) correlation with length of stity $(\mathrm{r}=16 ; \mathrm{p}<.001)$. However, in the eventual regression analysis this dummy variable has no significant effect anymore. The reasons for this are complicated. A high CPK enzyme release turns out to be correlated with pericarditis (PERIC: $\mathrm{T}=.25 ; \mathrm{p}<.(\mathrm{O}) 1$ ), mitral insufficiency (MITINS: $\mathrm{r}=.12 ; \mathrm{p}<.01$ ), a slightly impairment of the LVEF (DLVEFI:r=.11;p<.01) and sinustachycardia or atrial flutter/-fibrillations in the acute phase combined with ventricular flutter/-fibrillations or sustained ventricular tachycardia in follow-up phase of stay (DRHYTHM9:r=.12;p<.01). These severity indices turn out to be a better explanation for differences in length of stay than the CPK-index (table 7.19). Almost identical results are found when severity of myocardial necrosis is measured by SGOT or LDH enzymes. These results are therefore not presented here.

Within the set of dummy variables representing the severity of necrosis measured by thallium test the only variable significantly correlated with length of stay appears to be DNETHEXT1 (irreversible thallium changes in 1 or 2 wall segments: $r=09 ; p<.05$ ). In the eventual regression analysis the partial regression coefficient does not turn out to be significant. Thus, severity of myocardial necrosis measured by the thallium exercise test will have no consequences for the duration of the hospital stay.

It may be concluded that the severity of myocardial necrosis (measured by ECG, enzymes or thallium) has no substantial effect on the length of the hospital stay of AMI patients. 
Table 7.13 Mean length of stay of patients in categories representing myocardial necrosis estimated by total CPK release $(n=457)$.

\begin{tabular}{llrrr}
\hline Category & Description & Mean & Sid.dev & Freq. \\
0 & no sig. CPK changes & 5.89 & 5.11 & 9 \\
& $<10000$ & 9.75 & 6.70 & 85 \\
2 & $10000-20000$ & 11.07 & 4.99 & 72 \\
3 & $20000-40000$ & 10.87 & 3.97 & 105 \\
4 & $40000-60000$ & 11.20 & 5.53 & 59 \\
5 & $>60000$ & 13.70 & 7.20 & 56 \\
& missing & 8.44 & 6.48 & 71 \\
& & & & \\
Eta $2: .06(p<.001)$ & & & \\
\hline
\end{tabular}

\section{* Severity of ischemic pain.}

The severity of ischemic pain reported by the patient (see $\$ 6.2 .4$.) appears to have a significant correlation with the length of stay. Patients who have ischemic pain only in the acute phase of the stay (TO or $\mathrm{Tl}$ ) appear to have a significantly lower chance of staying in hospital longer (DISPAIN1:r=-.14;p<.(001). If patients also suffer from ischemic pain in a later phase of the stay (i.e. : after three days) the chance of staying in hospital longer will strongly increase (DISPAIN2: $\mathrm{r}=.34 ; \mathrm{p}<.001$ ). In total $13 \%$ of the non-linear variance in length of stay is explained by the severity of ischemic pain alone (table 7.14 ). In the eventual regression analysis, patients with ischemic pain after three days turn out to have significantly higher chances of staying longer in hospital (table 7.19).

It may be concluded that the severity of ischemic pain will increase the length of stay; patients who still have ischemic pain afier three days will generally stay longer in hospital.

Table 7.14 Mean length of stay of patients in categories representing severity of ischemic pain $(\mathrm{n}=457)$.

\begin{tabular}{|c|c|c|c|c|}
\hline Category & Description & Mean & Sid.dev & Freq. \\
\hline 0 & no ischemic pain & 7.82 & 6.44 & 54 \\
\hline$I$ & ischemic pain in TO or Tl & 10.02 & 4.89 & 306 \\
\hline \multirow[t]{2}{*}{2} & ischemic pain (also) in $T 2$ or $T 3$ & 14.68 & 6.88 & $9 !$ \\
\hline & missing & 3.67 & 2.94 & 6 \\
\hline$E t a^{2}: 13$ & $001)$ & & & \\
\hline
\end{tabular}

* Severity of Cardiac Wall Motion abnormalities (CWM).

Given the corrclations between the length of stay and three sets of dummy variables each representing the dimensions in severity of cardiac wall motion abnormalities (see 86.2 .5 .), the most important dimension appears to be the categories representing the extent of cardiac wall motion abnormalities. As can be seen from table 7.15 the mean length of stay of patients increases when more cardiac wall segments show any kind of wall motion abnormality. The variance explained exclusively by this dimension amounts to .12. The 
dummy variables representing the extent with significant correlations are: CWMEXT2 (CWM abn. in 3 wall segments; $r=.12 ; p<.01$ ), CWMEXT3. (CWM abn. in 4. wall segments: $r=.19 ; p<.001$ ) and CWMEXT4 (CWM abn. in 5 wall segments $: r=.19 ; p<.001$ ).

Variance explained by the intensity and location categories of cardiac wall motion abnor malities is somewhat lower, but still significant (Eta ${ }^{2}: 06 ; p<.05$ resp. .07;p<.001). However, in the eventual regression analysis the severity of cardiac wall motion abnormalities offers no additional explanation for length of stay. All three severity dimensions have been tested separately in regression analysis and none of them have a significant effect on the length of stay.

Why these effects are not significant anymore may be illustrated by the categories representing the extent of cardiac wall motion abnormalities. It turns out that if patients have CWM abnormalities in 3 wall segments, chances of having a LVEF between 30 and $40 \%$ (DLVEF1: $r=.24 ; p<.01$ ), of having pericarditis (PERIC: $r=.13 ; p<.01$ ), and of having recurrent sinustachycardia (DRHYTHM $5: \mathrm{r}=.14 ; \mathrm{p}<.01$ ) are significantly higher. If patients have CWM abnormalities in 4 wall segments chances of having an impaired LVEF increase (DLVEF1: $r=.19: p<.001$ and DLVEF2: $r=.21 ; p<.001$ ). Patients with CWM abnormalities in 5 wall segments appear to have significantly higher chances of suffering from mitral insufficiency (MITINS: $r=.15 ; p<.01$ ), from pericarditis (PERIC: $r=15 ; \mathrm{p}<001$ ), from rhythm abnormalities like recurrent atrial flutter/-fibrillations (DRHYTHM7: $r=.16 ; p<.01$ ) or from acute sinustachycardia or atrial flutter/-fibrillations combined with ventricular flutter/fibrillations or sustained ventricular tachycardia later on (DRHYTHM9:r=.15;p<.01). In addition, the mortality risk in a later stage of hospital admission is significantly higher for patients who have these extensive wall motion abnormalities (DDEATH $3: r=.15 ; p<.01$ ). As will be seen later on, in the eventual regression analysis these latter variables turn out to provide a better explanation of differences in length of stay than the extent of cardiac wall motion abnormalitics.

It may be concluded that the severity of cardiac wall motion abnormalities eventually has no substantial effect on the length of stay of AMI patients.

Table 7.15 Mean length of stay of patients in severity categories representing the extent of Cardiac Wall Motion abnormalities ( $n=457$ ).

$\begin{array}{llrrr}\text { Category } & \text { Description } & \text { Mean } & \text { Std.dev. } & \text { Freq. } \\ \text { 0 } & & & \\ 1 & \text { no wall motion abnormalities } & 9.45 & 2.85 & 22 \\ 2 & \text { wall motion abn. in I or 2 wall segments } & 10.49 & 4.27 & 126 \\ 3 & \text { wall motion abn. in 3 wall segments } & 12.19 & 5.56 & 88 \\ 4 & \text { wall motion abn. in 4 wall segments } & 14.42 & 6.27 & 38 \\ 5 & \text { wall motion abn. in 5 wall segments } & 15.80 & 6.20 & 20 \\ & \text { wall motion alm. in 6 or 7 wall segments } & 9.29 & 5.72 & 17 \\ & \text { missing } & 8.37 & 6.52 & 146\end{array}$

$E t a^{2}: 12(p<.0 O I)$ 
* Severity of cardiac rhythm disorders.

As can be seen from table 7.16, variance explained by categories comprising the cardiac rhythm disorders $(\S$ see 6.2 .6$)$ amounts to, 20 . The correlations between the dummy variables representing the categories of rhythm disorders and the length of stay are significant in four cases: when patients suffer from recurrent sinustachycardia or nonsustained ventricular tachycardia (DRHYTHM5: $r=.18 ; p<.001$ ), when patients have acute atrial flutter/-fibrillations at the beginning of their stay and sinustachycardia or nonsustained ventricular tachycardia in the follow-up phase (DRHYTHM6: $r=09: \mathrm{p}<.05$ ), when patients have recurrent atrial flutter/-fibrillations (DRHYTHM7:r=.21;p<.001) or when patients have sinustachycardia or atrial flutter/-fibrillations in the beginning combined with ventricular flutter/-fibrillations or sustained ventricular tachycardia in the follow-up phase (DRHYTHM9: $r=.28 ; \mathrm{p}<.001$ ).

In the eventual regression analysis the effects of five severity categories of rhythm disorders turn out to be significant (table 7.19). A relatively moderate increase in length of stay occurs for: (1) patients who have acute sinustachycardia or atrial flutter/-fibrillations combined with ventricular flutter/-fibrillations or sustained ventricular tachycardia in the follow-up phase (DRHYTHM9), (2) patients who have recurrent atrial flutter/-fibrillations (DRHYTHM7) and (3) patients who suffer from recurrent sinustachycardia or non-sustained ventricular tachycardia (DRHYTHM5). A relatively minor, but still significant increase in length of stay results for: (4) patients who have acute ventricular flutter/-fibrillations within 3 days followed by atrial flutter/fibrillations or sinustachycardia in the follow-up phase of their stay (DRHYTHM8) and (5) patients who only have acute sinustachycardia within the first three days of the stay (DRHYTHM1). The last (unexpected) effect can be explained because conditional dummy variables DDEATH1 and DDEATH3 are presumedly acting as suppressor variables on the original relation between DRHYTHMI and length of stay ( $r=$.$04 ; p=.18$ ). Patients who have sinustachycardia at the beginning of their stay tend to die sooner (in period T1), so their stay is relatively shon. At the same time, if patients with this rhythm disorder stay alive, they will have a longer hospital stay. This implies that the relationship between the occurrence of this rhythm disorder and length of stay tends to be obscured and underestimated by early decease during stay. If one corrects this, the relationship between severity of this rhythm disorder and length of stay turns out to be a positive and significant one. The effect caused by DRHYTHM 8 seems to occur by random chance only: the effect is only just statistically significant.

It may be concluded that the severity of cardiac rhythm disorders has a substantial increasing effect on the length of hospital stay for AMI patients; patients with (i) acute sinustachycardia (2) recurrent sinustachycardia or non-sustained ventricular lachycardia or (3) recurrent atrial fluttert-fibrillations or (4) acute ventricular flutter/-fibrillations combined with atrial flutter/-fibrillations or sinustachycardia in the follow-up phase or (5) acuse sinustachycardia or atrial flutterl-fibrillation in the beginning combined with ventricular flutter/-fibrillations or sustained ventricular tachycardia in follow-up phase will stay longer in hospital. 
Table 7.16 Mean length of stay of patients in severity categories representing type and time of appearance of cardiac rhythm disorders ( $\mathrm{n}=457$ ).

\begin{tabular}{|c|c|c|c|c|}
\hline Category & Description & Mean & Std.dev. & Freq. \\
\hline$a / 0$ & no rhythum disorder & 8.99 & 4.56 & 221 \\
\hline$b / l$ & acute sinustachycardia & 10.16 & 4.90 & 104 \\
\hline$c / 2$ & acute atrial flutter/-fibrillations & 11.24 & 4.15 & 21 \\
\hline$d / 3$ & acute ventricular flutter/-fibrillations & 9.76 & 5.55 & 17 \\
\hline$e / 4$ & $\begin{array}{l}\text { sinustachycardia or non-sustained ventricu- } \\
\text { lar tachycardia in follow-up phase }\end{array}$ & 12.17 & 5.31 & 18 \\
\hline$f / 5$ & $\begin{array}{l}\text { recurrent sinustachycardia or non-sustai- } \\
\text { ned ventricular tachycardia }\end{array}$ & 14.92 & 8.86 & 26 \\
\hline$g / 6$ & $\begin{array}{l}\text { acute } A F / F \text { combined with sinustachycardi- } \\
\text { a or non-sustained ventricular tachycardia } \\
\text { in follow-up phase }\end{array}$ & 15.00 & 6.54 & 6 \\
\hline 127 & recurrent $A F / F$ & 15.33 & 8.19 & 30 \\
\hline$i / 8$ & $\begin{array}{l}\text { acute VF/F combined with } A F / F \text { or sinus- } \\
\text { tachycardia in follow-up phase }\end{array}$ & 13.00 & 7.24 & 6 \\
\hline \multirow[t]{2}{*}{$j / 9$} & $\begin{array}{l}\text { acute sinustachycardia or } A F / F \text { combined } \\
\text { with VF/F or sustained ventricular tachy. } \\
\text { cardia in follow-up phase }\end{array}$ & 23.71 & 4.23 & 7 \\
\hline & missing & 1.00 & .00 & 1 \\
\hline \multicolumn{5}{|c|}{$E t a^{2}: .20(p<.001)$} \\
\hline \multicolumn{5}{|c|}{$A F / F=$ Atrial Flutter/-Fibrillations } \\
\hline \multicolumn{5}{|c|}{ VF/F $=$ Ventricular Flutter/-Fibrillations } \\
\hline
\end{tabular}

\section{* Severity of cardiac conduction disorders.}

As can be seen in table 7.17. the variance in length of stay explained by categories representing cardiac conduction disorders (see $\$ 6.2 .7$ ) amounts to $4 \%$. The only dummy variable that has a significant correlation with the length of stay appears to be DCONDUC5 (recurrent right bundle branch block $(\mathrm{RBBB}): \mathrm{r}=.13 ; \mathrm{p}<.05)$. However, in the eventual regression analysis the parameter belonging to this conduction disorder appears to be not significant. Patients who have a recurrent RBBB during their stay tend to have a severe impaired Left Ventricular Ejection Fraction (DLVEF2:r=.19;p<.001) and this will eventually cause a prolonged hospital stay. At the same time these patients tend to have sinustachycardia or atrial flutter/-fibrillations in the beginning combined with ventricular fluticr/-fibrillations or sustained ventricular tachycardia in the follow-up phase of stay (DRHYTHM9: $\mathrm{r}=.16 ; \mathrm{p}<.001$ ) and this will also result in a longer hospital stay. The third factor related to RBBB is pericarditis (PERIC: $\mathrm{r}=.13 ; \mathrm{p}<. .01$ ) and this factor tends to explain apart of the assumed effect of RBBB on the length of hospital stay too.

It may be concluded that the severify of cardiac conduction disorders has no substantial effect on the length of stay of AMI patients. 
Table 7.17 Mean length of stay of patients in severity categories representing type and time of appearance of cardiac conduction disorders $(n=457)$.

\begin{tabular}{|c|c|c|c|c|}
\hline Category & Description & Mean & Siddev: & Freq. \\
\hline$a / 0$ & no conduction disorder & 10.56 & 5.78 & 366 \\
\hline$b / l$ & acute Mobitz I AV-block & 11.27 & 2.65 & 11 \\
\hline$c / 2$ & $\begin{array}{l}\text { acute Mobitz II AV-block or acute third } \\
\text { degree block with nodal escape rhytlom }\end{array}$ & 10.78 & 6.34 & 36 \\
\hline$d / 3$ & acute right bundle branch block & 7.82 & 6.43 & II \\
\hline$e / 4$ & $\begin{array}{l}\text { acute thind degree block with ventricular } \\
\text { escape rhythm }\end{array}$ & 6.55 & 5.22 & II \\
\hline$f / 5$ & recurrent right bundle branch block & 14.33 & 7.84 & 18 \\
\hline$g / 6$ & $\begin{array}{l}\text { third degree block combined with ventri- } \\
\text { cular escape rhythm in follow-up phase }\end{array}$ & 13.75 & 6.95 & 4 \\
\hline
\end{tabular}

$\operatorname{Eta}^{2}: .04(p<.05)$

\section{* Severity of heart failure.}

The severity of heart failure of patients (see $\$ 6.2 .8$. i.) measured by the Left Ventricular Ejection Fraction (LVEF) is positively and significantly related to their length of stay. Variance explained by this indicator alone amounts to 17 (table 7.18). The dummy variables representing the LVEF severity categories that show a positive significant correlation with the length of stay are DLVEF1 $(r=.24 ; p<.001)$ and DLVEF2 $(r=.27 ; p<.001)$. Sirangely enough, scores on LVEF lower than $20 \%$ seems to have no correlation to length of stay (DLVEF3:r=-.056; $p=.12$ ). This may be explained by the high mortality risk of these patients within ten days; there is a moderately high correlation between this dummy variable and DDEATH2 $(r=.29 ; p<.001)$. This factor is also correlated 10 MITINS (mitral insufficiency: $r=.17 ; p<.001$ ) and DRHYTHM5 (recurrent sinustachycardia: $r=.20 ; p<.(0) 1$ ). For patients with an LVEF between $20 \%$ and $30 \%$ (DLVEF2) this effect is also not significant. This can be explained because these patients tend to have higher chances of dying in a later phase (DDEATH3: $r=25 ; \mathrm{p}<.001$ ), of having pericarditis (PERIC:r=.17; $p<.001$ ), of having anteroseptal ischemia (ISLOC: $r=.19 ; p<.001$ ) and two types of cardiac rhythm disorders, i.e. recurrent atrial flutter/-fibrillations and sinustachycardia or atrial flutter/-fibrillations in the beginning combined with ventricular flutter/fibrillations or sustained ventricular tachycardia in the follow-up phase (DRHYTHM7: $r=.17 ; p<.001$, resp. DRHYTHM9: $r=.31 ; p<.001)$ ). The only significant dummy variable in the eventual regression analysis is DLVEFI (table 7.19). This means that if patients have an LVEF between $30 \%$ and $40 \%$, chances of staying longer in hospital will increase significantly.

The severity of heart failure measured by the Left Ventricular End Diastolic Dimension (LVEDD) is positively correlated with the length of stay $(r=.15 ; p<.0() 1)$. However, in the eventual regression analysis the LVEDD has no significant effect on the length of stay. This may be explained by the correlations this factor has with the LVEF dummy variables (DLVEF1:r=.11; $<<.05$, DLVEF2: $r=.28 ; p<.001$ and DLVEF $3: r=15 ; p<.001$ ).

The severity of right sided heart failure (see $\$ 6.2 .8 .2$.$) , measured by the collapse of the$ Vena Cava Inferior (the VCI-index) shows a weak positive correlation with the length of stay (VCI:r $=13 ; p<.05)$. Patients with a VCI index less than $50 \%$ seem to have a higher chance of staying in hospital for a longer period of time. However, the effect is not significant in the eventual regression analysis. Patients with VCI lower than $50 \%$ have more 
chances of being in a. LVEF category lower than $30 \%(\mathrm{r}=.16 ; \mathrm{p}<.001)$, and are more likely to suffer from typical cardiac rhythm disorders, like recurrent atrial flutter/-fibrillations (DRHYTHM7: $r=.13 ; \mathrm{p}<.01$ ) or acute ventricular flutter/-fibrillations combined with atrial flutter-/fibrillations or sinustachycardia later on (DRHYTHM8; $r=12 ; p<.01$ ). Furthermore, these patients show a higher probability to have mitral insufficiency $(r=.13 ; \mathrm{p}<.001)$. All these factors have a direct effect on the length of stay, and the assumed effect of VCI on length of hospital stay will be explained by these deficiencies.

The correlations of right ventricular infarction, measured both by ECG ST-changes as well as by abnormal wall motion with length of stay appear not to be significant (ISRV: $r=.07 ; p=.09$ and CWMRV:r=.06; $=.13$ ). In the eventual regression analysis these factors have no substantial effect on the length of stay at all.

It may be concluded that the severity of left ventricle heart failure, measured by LVEF, has a substantially increasing effect on length of hospital stay; patients with an LVEF between 30 $\%$ and $40 \%$ will stay in hospital longer. Right-sided heart failure will not result in a longer hospital stay.

Table 7.18 Mean length of stay of patients in severity categories representing the Left Ventricular Ejection Fraction $(\mathrm{n}=457)$.

$\begin{array}{llrrr}\text { Category } & \text { Description } & \text { Mean } & \text { Std.dev. } & \text { Freq. } \\ & & & & \\ 0 & \text { normal }(L V E F>40 \%) & 9.97 & 3.37 & 183 \\ 1 & \text { slighluly impairment }(30 \%<L V E F<40 \%) & 13.77 & 6.09 & 79 \\ 2 & \text { severe impaiment }(20 \%<L V E F<30 \%) & 15.29 & 7.06 & 48 \\ 3 & \text { very severe impairment }(L V E F<20 \%) & 8.13 & 6.60 & 8 \\ & \text { missing } & 8.17 & 6.44 & 139\end{array}$

$\operatorname{Era}^{2}: .17(p<.001)$

\section{* Severity of physical deficiencies and specific cardiac complications.}

Physical deficiencies (see $\$ 6.2 .9$.) correlated with the length of stay are palpitations (PALP:r=.16:p<.001), pre-collapse (PRECOL: $r=.21: p<.001)$, syncope/collapse (SYCOL: $\mathrm{r}=15 ; \mathrm{p}<.(0) 1$ ), dyspnea (DYSP: $\mathrm{r}=.26 ; \mathrm{p}<.(0) 1$ ), edema in the lower abdomen or in the extremities (BEDEMA: $=10 ; p<.05)$ and lung edema (LEDEMA:r=15;p<.01). In the eventual regression analysis only pre-collapse offers an additional explanation of the duration of hospital stay (table 7.19). The parameters of all other physical deficiencies significantly correlated with the length of stay turn out to be non-significant in the eventual regression analysis. For instance, patients who have lung edema have a higher mortality risk: in period $\mathrm{T} 3$ (after ten days). Impairment of cardiac function is highly correlated with lung edema and this turns out to be one of the genuine factors influencing a longer stay. Patients having palpitations during their stay are more likely to suffer from specific rhythm disorders. like recurrent atrial flutter/ -fibrillations (DRHYTHM7:r=.18; $<<.01$ ) or atrial flutter/ fibrillations or sinustachycardia in the first part of their stay combined with ventricular flutter/-fibrillations or sustained ventricular tachycardia later on in their stay (DRHYTHM9: $r=.21: p<.01$ ). In addition to all this, the probability that these same patients have pericarditis increases (PERIC: $\mathrm{r}=13 ; \mathrm{p}<.01$ ) and they have a higher probability to die during period T3 (DDEATH3: $\mathrm{r}=.13 ; \mathrm{p}<.01$ ). 
Patients suffering from syncope/collapse tend to have atrial flutter/-fibrillations or sinustachycardia in the first part of their stay combined with ventricular flutter/-fibrillations or sustained ventricular tachycardia later on in their stay (DRHYTHM9: $r=.25 ; p<.01$ ). Patients with syncope/collapse also have more chance of suffering from mitral insufficiency (MITINS: $r=.19 ; \mathrm{p}<.01$ ) and/or CVA/TIA (CVA: $r=.21 ; p<.01)$.

Having dyspnea during stay is (cor)related to many factors in the medical model, which tend to explain the moderately positive correlation between dyspnea and length of stay. Dyspnea patients will have more anteroseptal myocardial ischemia (ISLOC: $r=.25 ; p<.01$ ), tend to suffer from ischemic pain in the later part of their stay (DISPAIN2: $r=.34 ; p<.001$ ) and will have cardiac rhythm abnormalities like recurrent atrial flutter/-fibrillations (DRHYTHM7: $\mathrm{r}=.21 ; \mathrm{p}<.01$ ). Also LVEF-score deviations tend to be higher in these patients (DLVEF1: $r=.24 ; p<.01$ and DLVEF $2: r=.27 ; p<.01$ ), and they will have a higher probability to die during period T3 (DDEATH3: $r=.28 ; p<.001$ ).

Patients with body edema are likely to have cardiac rhythm abnormalities like recurrent atrial flutter/-fibrillations (DRHYTHM7: $r=.21 ; p<.01$ ), to have high deviations on the LVEF score (DLVEF2: $r=.27 ; p<.01$ ) and to have mitral insufficiency (MITINS: $r=.29 ; p<.001$ ). These same patients will also have higher chances of dying within period T3 (DDEATH3: $r=.28 ; p<.001$ ).

Other cardiac complications correlated with the length of stay are: mitral insufficiency (MITINS; $r=.19: p<.05)$, CVA/TIA $(\mathrm{CVA}: \mathrm{r}=.21 ; \mathrm{p}<.001)$ and pericarditis (PERIC: $\mathrm{r}=.27$; $\mathrm{p}<.001)$. All assumed effects of these factors are also significant in the eventual regression analysis (table 7.23). Cardiogenic shock shows a significant negative correlation with hospital stay (CSHOCK:r=-.23:p<.001). This correlation may be explained by the high mortality rate of these patients which is considerable (in $T 0: r=44$, in $T I: r=47$ and in $T 2$ : $\mathrm{r}=.44$ ). Dying in hospital during these periods is presumedly the reason why this type of complication offers no additional explanation in the eventual regression analysis.

It may concluded that some typical physical deficiencies and cardiac complications have a substantial effect on the length of stay; patients with pre-collapse or pericarditis, mitral insufficiency or CVAITIA will stay longer in hospital.

\section{* Characteristics of admission and discharge.}

From the analysis it may be concluded that, apart from the manifold effects of the patient's severity of illness conditional factors like type of admission and type of discharge are significantly related to the length of stay: patients coming from other hospitals have a shorter length of stay, if patients are transferred to other medical departments their total length of stay will increase and if patients die in hospital within ten days their overall length of stay is of course shorter. If patients die after ten days their length of hospital stay will however increase importantly.

\section{* Background characteristics.}

From all individual background characteristics (see $\$ 6.3 .1$ ) the only variables showing significant correlations with the length of stay are: AGE $(r=14 ; p<.01)$ and SEX $(r=14 ; p$ $<.01)$. In the eventual regression analysis the parameters belonging to these variables turn out to be non-significant. Generally speaking, sex and age are correlated with one or more severity indicators: the older the patients the more severe their illness is and vice versa. Age is sometimes assumed to be a reliable predictor for the length of stay. For the patient population under survey only $2 \%$ of differences in length of stay can be explained that way. When a difference is made between deceased and non-deceased patients the age seems to be more relevant in the deceased group (LOS: $\mathrm{r}=.36 ; \mathrm{p}<.001$ ). Patients who die in hospital after a prolonged stay appear to be much older than patients who die acutely. 
It may concluded that individual background characteristics of patients have no extra predictive value for the length of stay, given their severity of illness.

Table 7.19 Results from the eventual regression analysis: dependent variable is total length of stay in hospital for AMI-patients (Listwise deletion of missing cases, $n=454$ ).

\begin{tabular}{|c|c|c|c|c|}
\hline Independent Variables & $B$ & $S E B$ & Beta & $\operatorname{Sig} T$ \\
\hline ISLOC & .70 & .25 & .07 & .006 \\
\hline DISPAIN & -1.13 & 1.49 &. .02 & NS \\
\hline DISPAINI & .78 & .50 & .06 & NS \\
\hline DISPAIN2 & 2.34 & .57 & .16 & .000 \\
\hline DRHYTHMI & 1.08 & .35 & .08 & .002 \\
\hline DRHYTHM2 & .17 & .69 &.$\infty$ & NS \\
\hline D)RHYTHM 3 & .02 & .78 & .00 & NS \\
\hline DRHYTHMA & 1.30 & .71 & .04 & NS \\
\hline IRHYTHMS & 2.37 & .64 & .09 & .000 \\
\hline DRHYTHMO & 1.79 & 1.32 & .03 & NS \\
\hline IКНКТНМ7 & 3.18 & .60 & .13 & .000 \\
\hline DRHYTHM 8 & 2.47 & 1.25 & .05 & .049 \\
\hline I)RНYTHMQ & 5.03 & 1.28 & .10 & .000 \\
\hline DECHO & .06 & .34 & .00 & NS \\
\hline I)LVMIS & 3.08 & 3.22 & .02 & NS \\
\hline DLVEFI & 1.40 & $.4 I$ & .09 & .001 \\
\hline DLVEF2 & .55 & .54 & .03 & NS \\
\hline DLVEF3 & -1.88 & 1.23 & -.04 & NS \\
\hline PRECOL & 1.40 & .57 & .06 & .014 \\
\hline PERIC & 2.28 & .50 & .11 & .000 \\
\hline MITINS & 1.10 & .43 & .06 & .011 \\
\hline$C V A$ & 3.59 & 1.34 & .08 & .008 \\
\hline IAAINTO & 3.19 & .84 & .09 & .000 \\
\hline I)AIDEXTI & -1.63 & 1.36 & -.03 & NS \\
\hline DADEXT2 & -2.94 & 1.10 & .07 & .008 \\
\hline DADEXT3 & -8.83 & 1.74 & -12 & .000 \\
\hline I HOMEI & -4.71 & 1.22 & .09 & .000 \\
\hline DHOME3 & 5.23 & .35 & .42 & .000 \\
\hline ITRANSI & 4.03 & 2.14 & -.05 & NS \\
\hline DTRANS2 & 21.08 & 1.87 & .29 & .000 \\
\hline DTRANS3 & 12.74 & 2.87 & .10 & .000 \\
\hline DIDEATHO & -6.32 & 1.11 & -15 & .000 \\
\hline DDEATHI & -5.94 & .75 & .20 & .000 \\
\hline DDEATH2 & -4.40 & .89 & -13 & .000 \\
\hline DDEATH 3 & 11.39 & 1.24 & .24 & .000 \\
\hline CONSTANT & 6.68 & 1.51 & & .000 \\
\hline \multicolumn{5}{|c|}{ Eyplained Variance $\left(R^{2}\right): .79$} \\
\hline \multicolumn{5}{|c|}{$\begin{array}{l}B=\text { unstandardized (partial) regression coefficient: } S E B=\text { standard error belonging to } B: B E T A= \\
\text { standandized (partial) regression coefficient: } S I G T=\text { significance of } B \text { or } B E T A \text { according to the } \\
T \text {-distribution } /(\text { degree of freedom) }\end{array}$} \\
\hline
\end{tabular}




\section{Discussion.}

It may be concluded that in patients with AMI, the severity of illness will significantly increase the length of stay at the hospital. Relevant severity indicators contributing each to an increase in length of stay are (1) anteroseptally located ischemia, (2) prolonged ischemic pain after three days of hospital stay, (3) specific cardiac rhythm disorders, (4) a LVEF-score between $30 \%$ and $40 \%$, (5) pre-collapse, (6) pericarditis, (7) mitral insufficiency and (8) CVAVTIA. The variance explained by these severity indicators is $46.9 \%$.

In almost all cases the effects presumed from other severity indicators that have significant correlations with the length of stay but that have non-significant parameters in the regression analysis turn out to be explained by the correlations with one or more medical deficiencies. The only exception to this is the presumed effects of a LVEF-score lower than $20 \%$ and cardiac shock which become non-significant because of the correction for deceased patients. So, these severity indicators still might be seen as factors indirectly causing a shorter length of stay. Inclusion of the "death" variables next to the severity of illness indicators increases the variance explained to $59.8 \%$. From the analysis it turns out that patients who die within ten days have a very shor length of stay. But patients who die after ten days will have an extremely long length of stay.

The additional variance next to severity of illness explained by the type of admission and transfer turns out to be low (total is $48.1 \%$ resp. $53.0 \%$ ). When 'transfer' variables are included in the regression analysis, they turn out to have a decreasing effect on the regression coefficient of CVA/TIA. This complication will increase the length of stay at the cardiology department with about four days while the overall length of stay increases by approximately ten days.

Next to the severity indicators introduction of other forms of non-regular discharge (DHOMEl and DHOME3) will increase the variance explained to $58.9 \%$. None of the above mentioned severity indicators become non-significant in the eventual regression analysis, except for two types of cardiac rhythm disorders (DRHYTHM4 and DRHYTHM6). In the eventual analysis these effects are interpreted as spurious (patients are expected to have typical complications not included in this study), but some caution is still in place. These two cardiac rhythm disorders might have substantial elfects on lenght of stay. But this can only be established if other factors influencing the length of stay, which we have not measured in this study, are included in the dalia-analysis.

When the DHOME-variables are introduced in the last step of the regression analysis the value of some regression coefficients of severity indicators like ISLOC (first B=1.4 now $B=0.7$ ), DISPAIN2 (first $B=4.1$ now 2.4) and DRHYTHM8 (first $B=4.9$ now 2.5 ) turns out to decrease. All other indicators show only a minor decrease in their regression coefficients. For the time being, the present solution involving a model in which the regression coefficients are subjected to a strict form of controll is preferred in spite of the risk of underestimating the effects of some indicators of severity in illness. In total $79 \%$ of the variance in length of stay is predicted by the severity of illness and conditional factors together. The relative importance of these variables on the length of stay (beta parameters) is presented in diagram 7.20 . 
Diagram 7.20 Path-analytic model in which an explanation is given of total length of hospital stay in patients having AMI (Listwise deletion of missing cases, $n=454$ ).

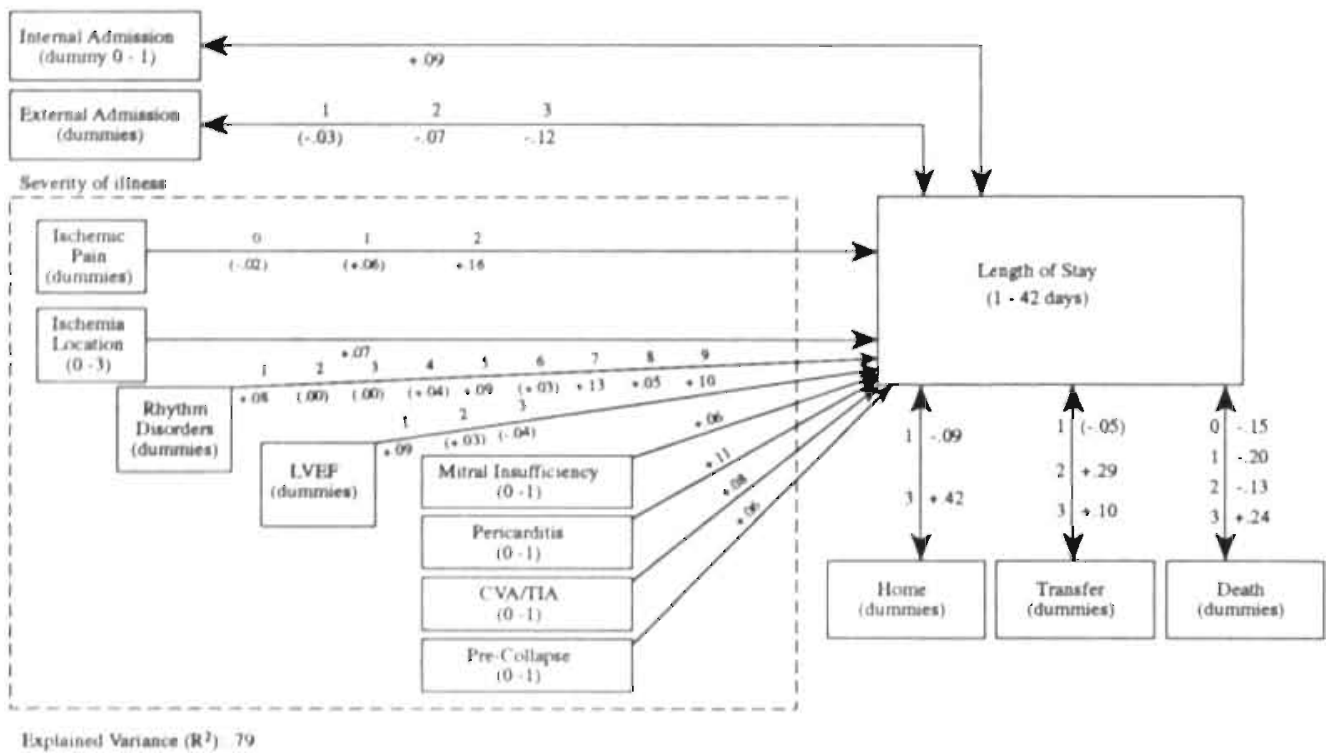

Liffects of dummy variables in brackets are not significant at the $5 \%$ level. Only beta parameters are shown.

\subsubsection{Explanation of costs of the coronary care unit.}

The costs of the coronary care unit of a patient are defined as the average material and personnel costs per CCU-day multiplied by the number of days the patient is admitted to the $\mathrm{CCU}$ (see $\$ 6.1 .3$.). For the total patient population of 464 patients the mean CCU costs amount to 1398.27 guilders (std.dev. = 1572.43); costs vary between 0.00 and 15074.80 guilders. Three patients have heen excluded from the analysis because of their extremely high $C C U$ costs $(m=11016.20$, sid.dev. $=3701.20)$. The mean $C C U$ cost of the remaining patients is 1335.68 guilders ( $\mathrm{std}$.dev $=1349.77 ; n=461$ ); costs now vary between 0.00 and 8407.10 guilders. Correlations between severity of illness, background characteristics, length of stay and CCU costs are presented in appendix 21.

\section{* Severity of coronary stenosis.}

Some correlations between CCU costs and the dummy variables representing the intensity, extent and location of coronary stenosis (see $\$ 6.2 .1$ ) appear to be statistically significant. If we resinict ourselves to correlations higher than 1.101 , three of them seem to be relevant. Paticnts having such a decrease in stenosis that no further deficiencies can be detected dering stay appear 10 have higher CCU costs (DSTENEXT1:r=.12;p<.01). Similarly. patients with decreasing scores on the location index to about 'normal' values seem to have higher CCU cosis (DSTENLOC1:r=.14:p<.01). The third correlation concerns the one between having a heart catheterization during stay and the CCU costs; having a catheterization appears to increase the $\mathrm{CCU}$ costs. When dummy regression analysis of CCU costs is done using each of the three stenosis indices separately, only patients who have decreasing scores on the index of location of stenosis appear to incur significantly more CCU costs, even if we control for type of stay (i.c. type of admission and type of discharge in hospital). However, this 'effect' is explained by the enrollment in the PRIMI-protocol; if. patients participate in this clinical trial they receive a catheterization twice. It is assumed that. 
this is the reason why they are kept under prolonged observation in CCU. The enrollment in PRIMI itself appears to have no relation with the CCU costs.

Therefore, it may be concluded that the severity of coronary stenosis has no substantial effect on the CCU costs.

\section{* Severity of myocardial ischemia.}

The extent of myocardial ischemia measured by ECG at rest (see $\$ 6.2 .2 .1$.) appears to be moderately correlated with CCU costs, (ISEXT: $r=.18 ; p<.001)$, and if the ischemia is located in the anteroseptal wall segment of the left ventricle CCU costs will rise substantially (ISLOC: $r=.32 ; p<.001$ ). In the eventual. regression analysis only the location of myocardial ischemia provides an additional explanation for differences in CCU costs (table 7.26). If patients tend to have a high score on the location index of myocardial ischemia, they will have higer CCU costs. As one can see from the correlation matrix in appendix 21 , the extent of ischemia appears to be highly correlated with the location of ischemia $(r=.37 ; \mathrm{p}<.001)$.

We can reasonably assume that the severity of myocardial ischemia measured by exercise test will have no effect on the CCU costs. These tests are normally planned just before the patient leaves the hospital and are certainly not performed when a patient is staying at the CCU.

It may be concluded that the location of myocardial ischemia has a substantial effect on CCU costs; patients with ST-changes corresponding with the anteroseptal wall segment will have higher $C C U$ costs.

\section{* Severity of myocardial necrosis.}

According to appendix 21 , the severity of myocardial necrosis (see $\$ 6.2 .3 .1$.) measured by the ECG at rest shows moderate correlations with CCU costs for both the extent of necrosis (NECEXT: $r=18 ; p<.001$ ) and the location of necrosis (NEC.LOC: $r=.30 ; p<.001$ ). However, in the eventual regression analysis, both effects turn out to be non-significant. The extent of necrosis appears to be correlated with the extent of myocardial ischemia $(\mathrm{r}=.22 ; \mathrm{p}<.(0) 1)$. Similarly, the location of the necrosis appears to be very highly correlated with the location of cardiac wall motion abnormalities $(r=61 ; p<.001)$.

As can be seen from table 7.21 variance explained by the severity of myocardial necrosis measured by CPK-severity categories (see $\$ 6.2 .3 .2$ ) amounts 10.18 . Patients with a relatively low CPK release tend to have a shorter CCU-stay with low costs (DCPK $1: r=-.15 ; \mathrm{p}<.01$ ). This is, however, not confirmed in the eventual regression analysis. If patients have a very high CPK release, chances of staying in the CCU resulting in relatively high CCU costs increase (DCPK4:r=.13;p<.01 and DCPK5:r=.36;p<.(0)1). The CPK relcase (DCPK4 and DCPK5: area measure $>40.000$ ) appears to have an significant increasing effect on CCU costs in the eventual regression analysis (table 7.26). Myocardial neciosis measured by the other enzymes (SGOT and LDH) shows about the same correlations with CCU costs, but these factors offer no additional explanation for costs like CPK does, even when included separately in the same analysis.

It may concluded that the severity of myocardial necrosis has a substantial effect on the $C C U$ costs. The indicator explaining $C C U$ costs is the CPK enzyme release; patients who have $a$ high enzyme release of more than 40.000 ( $U /$ *hours) will have higher $C C U$ costs. 
Table 7.21 Mean CCU costs of patients in categories representing myocardial necrosis estimatied by total CPK release $(n=461)$.

$\begin{array}{ll}\text { Category } & \text { Description } \\ 0 & \text { no sig. CPK changes } \\ 1 & <10000 \\ 2 & 10000-20000 \\ 3 & 20000-40000 \\ 4 & 40000-60000 \\ 5 & >60000 \\ & \text { missing }\end{array}$

Mean

612.01

907.22

1120.42

1292.13

1807.04

2650.51

801.25
Std dev.

$\begin{array}{rr}686.71 & 9 \\ 789.87 & 85 \\ 896.84 & 74 \\ 980.65 & 105 \\ 1652.35 & 60 \\ 2025.48 & 56 \\ 1073.18 & 72\end{array}$

$E_{t a}^{2}: .18(p<.001)$

\section{*. Severity of ischemic pain.}

Severity measured by ischemic pain (see $\$ 6.2 .4$ ) appears not to be significantly correlated with the CCU costs. No significant correlations are found if patients have only ischemic pain in the acute phase of the stay (DISPAINI: $r=07 ; p=.08$ ) or if patients apart from this initial pain, sulfer from pain after three days of hospital stay (DISPAIN2: $r=06 ; p=.09$ ). In the eventual regression analysis it turns out that patients with ischemic pain do not stay in the CCU longer than patients without ischemic pain.

It may concluded that the severity of ischemic pain of patients has no substantial effect on the stay or $\mathrm{CCU}$ costs.

\section{* Severity of Cardiac Wall Motion abnormalities (CWM).}

The importan dimension in the severity of cardiac wall motion abnormalities as measured by the intensity, the extent and the location (see $\$ 6.2 .5$ ) appears to be the location scale. Variance in $\mathrm{CCU}$ costs explained by this indicator alone amounts to .12 (table 7.22). In dummy regression analysis (which includes also patients who do not receive an echocardiography) the variance explained amounts to .18. Patients appear to have much higher CCU costs if they have wall motion abnormalities in the anteroseptal wall segment of the left ventricle (DCWMLOC $1: r=.40 ; \mathrm{p}<.001$ ). This effect remains significant in the eventual regression analysis (table 7.26). Variance explained by the intensity-index of wall motion abnormalities alone amounts to only .03 (if patients who do not have echocardiography are excluded from analysis). In dummy regression analysis $(n=461)$ the explained variance of this factor alone amounts to .10. In the eventual regression analysis the assumed effect of this factor was not significant anymore: this can partly be explained by the fact that patients who show typical akinetic or dyskinetic cardiac wall motion tend to have a low left ventricular ejection fraction (correlations of DCWMINTI with DLVEF1 and DLVEF2 are resp. 26 and .38 , both significant at $\mathrm{p}<.001)$ and these last factors do explain CCU costs better. The non-significance in the effect of the intensity dimension of CWM abnormalities is furthermore explained by the simultaneous emergence of typical cardiac rhythm disorders: paticnts who have akinetic or dyskinetic wall motion abnormalities tend to suffer somewhat more from recurrent sinustachycardia or non-sustained ventricular tachycardia during stay (DRHYTHM5: $r=.10: p<.05$ ) and they are more likely to have sinustachycardia or atrial fluter/fibrillation in the beginning combined with left ventricular flutter/-fibrillations or sustained ventricular tachycardia in the later phase of their stay (DRHYTHM9: $r=.13 ; p<.01$ ). Due to the complications mentioned above the intensity-dimension of cardiac wall motion abnormalities has no significant effect on CCU costs in the eventual regression analysis. 
Variance explained by the extent of cardiac wall motion abnormalities alone amounts to .10 $(n=313)$. In dummy regression analysis $(n=461$, with inclusion of patients who do not have echocardiography) the variance explained amounts to .17 . The effects of the first two dummy variables (DCWMEXT1 and DCWMEXT2) are not significant, but the three higher numbered ones representing four wall segments and more seem to have significant effects on the CCU costs. In the eventual regression analysis these seemingly important effects are actually explained by the same factors mentioned in the preceding analysis: this is hardly surprising, since there appears to be a strong positive correlation between the intensity and extent scales $(r=.59 ; \mathrm{p}<.00 ; \mathrm{n}=313)$.

It may be concluded that the severity of cardiac wall motion abnormalities has a substantial effect on the costs of patients in the CCU. The best indicator proves to be the location of wall motion abnormalities; patients with wall motion abnormalities in the anteroseptal side of the left ventricle will have higher CCU costs.

Table 7.22 Mean CCU cosLs of patients within severity categories representing the location of Cardiac Wall Motion abnorrnalities $(n=461)$.

$\begin{array}{llrrr}\text { Category } & \text { Description } & \text { Mean } & \text { Stdidev: } & \text { Freq. } \\ & & & & \\ 0 & \text { no wall motion abnormalities } & 1041.01^{\prime} & 570.47 & 22 \\ 1 & \text { non-anteroseptal wall motion abnormalities } & 1184.35 & 913.59 & 164 \\ 2 & \text { anteroseptal' wall motion abnormalisies } & 2214.20 & 1953.15 & 127 \\ & \text { missing } & 793.31^{\prime} & 676.60 & 148\end{array}$

$E t a^{2}: .12(p<.001)$

* Severity of cardiac rhythm disorders.

As can be seen from table 7.23 variance of CCU costs explained by the severity of cardiac rhythm disorders ( $\sec \S 6.2 .6$ ) alone amounts to .34. The correlations between the dummy variables representing the various severity categories and the CCU costs are significant for four variables: (1) sinustachycardia or non-sustained ventricular tachycardia in the follow-up phase of stay (DRHYTHM4:r=.16;p<.001), (2) recurrent sinustachycardia or non-sustained ventricular tachycardia (DRHYTHM5:r=.22; $<<.001$ ), (3) acute ventricular flutter/. fibrillations followed by atrial flutter/-fibrillations or sinustachycardia (DRHYTHM 8:r=.13;p<.01) and (4) acute sinustachycardia or atrial flutter/-fibrillations combined with ventricular Mutter/fibrillations or sustained ventricular tachycardia in the follow-up phase of stay (DRHYTHM9: $r=.45 ; p<.001$ ). Patients with these specific rhythm disorders have a significantly higher chance of incurring high $\mathrm{CCU}$ costs. The supposed effects belonging to these rhythm abnormalities turn out to be significant in the eventual regression analysis (table 7.26 ).

It may concluded that specific types of cardiac rhythm disorders have substantial effects on the CCU costs; patients with (1) recurrent sinustachycardia or non-sustained ventricular iachycardia, or (2) sinustachycardia or non-sustained tachycardia in follow-up phase, or (3) acute $V F / F$ combined with $A F / F$ or sinustachycardia in follow-up phase, or (4) acute sinustachycardia or AF/F combined with VF/F or sustained ventricular tachycardia in follow-up phase will have higher CCU costs. 
Table 7.23 Mean CCU costs of patients in severity categories representing type and time of appearance of cardiac rhythm disorders $(n=461)$.

\begin{tabular}{|c|c|c|c|c|}
\hline Category & Description & Mean & Std dev. & Freq. \\
\hline$a / 0$ & no rhytlum disorder & 878.84 & 670.10 & 222 \\
\hline$b / l$ & acute sinustachycardia & 1339.06 & 1132.37 & 105 \\
\hline$c / 2$ & acute atrial flutter/-fibrillations & 1408.09 & 1149.72 & 21 \\
\hline d/3 & acute ventricular flutter/-fibrillations & 1127.39 & 937.48 & 18 \\
\hline$e / 4$ & $\begin{array}{l}\text { sinustachycardia or non-sustained } \\
\text { ventricular tachycardia in follow-up } \\
\text { phase }\end{array}$ & 2399.73 & 1886.14 & 18 \\
\hline$f / 5$ & $\begin{array}{l}\text { recurrent sinustachycardia or non- } \\
\text { sustained ventricular tachycardia }\end{array}$ & 2564.50 & 1936.38 & 26 \\
\hline$g / 6$ & $\begin{array}{l}\text { acute } A F / F \text { combined with sinustachy- } \\
\text { cardia or non-sustained ventricular } \\
\text { tachycardia in follow-up phase }\end{array}$ & 2029.30 & 2425.48 & 6 \\
\hline $1 / 7$ & recurrent $A F / F$ & 1517.14 & 1333.24 & 30 \\
\hline$i / 8$ & $\begin{array}{l}\text { acute } V F / F \text { combined with } A F / F \text { or } \\
\text { sinustachycardia in follow-up phase }\end{array}$ & 2733.34 & 2055.75 & 7 \\
\hline \multirow[t]{2}{*}{ j/9 } & $\begin{array}{l}\text { acute sinustachycardia or } A F / F \text { combined } \\
\text { with } V F / F \text { or sustained ventricular tachy- } \\
\text { cardia in follow-up phase }\end{array}$ & 6170.72 & 1549.58 & 7 \\
\hline & missing & 289.00 & 0.00 & I \\
\hline \multicolumn{5}{|c|}{$E t a a^{t}: 34(p<.001)$} \\
\hline \multicolumn{5}{|c|}{ AF/F $=$ Atrial Flutter/-Fibrillations } \\
\hline \multicolumn{5}{|c|}{$V F / F=$ Ventricular Flutter/-Fibrillations } \\
\hline
\end{tabular}

\section{* Severity of cardiac conduction disorders.}

Variance of $\mathrm{CCU}$ costs explained by cardiac conduction disorders (see $\S 6.2 .7$.) alone amounts 10.06 (table 7.24). Two conduction severity categories appear to be relevant with respect to CCU costs: recurrent righi bundle branch block (DCONDUC5: $r=.15 ; p<.001$ ) and third degree AV-block combined with ventricular escape rhythm in the follow-up phase (DCONDUC6: $\mathrm{r}=16 ; \mathrm{p}<.001)$. However, in the eventual regression analysis only the first lype of conduction disorders appears to have a significant effect (table 7.26). Why the assumed effect of the other type (DCONDUC6) is non-significant is partly explained by the fact that patients who have high levels of total CPK enzyme release (DCPK5) typically develop a third degree AV-block combined with ventricular escape rhythm in the follow-up phase $(r=.18 ; p<.001)$, and these enzyme values turn out to have a substantial increasing effect on the CCU costs. Furthermore, this conduction disorder tends to be accompanied by acute sinustachycardia or atrial flutter/fibriltations combined with ventricular flutter/fibrillations or sustained ventricular tachycardia in the follow-up phase (DRHYTHM9: $\mathrm{r}=.18: \mathrm{p}<.001$ ) or acute ventricular flutter/-fibrillations followed by atrial flutter/fibrilhtions later on (DRHYTHM8: $r=.18 ; \mathrm{p}<.001$ ). These two factors provide a better explanation for CCU cosis.

It may concluded that a specific type of cardiac conduction disorder will have a substantial effect on the CCU costs: patients with recurrent right bundle branch block will have subsrantially higher CCU costs. 
Table 7.24 Mean CCU costs of patients in severity categories representing type and time of appearance in cardiac conduction disorders $(n=461)$.

\begin{tabular}{|c|c|c|c|c|}
\hline Category & Description & Mean & Stddev: & Freq. \\
\hline$a 0$ & no conduction disorder & 1247.35 & 1236.23 & 370 \\
\hline$b / I$ & acute Mobitz I AV-block & 1027.83 & 901.62 & II \\
\hline$c / 2$ & $\begin{array}{l}\text { acute Mobitz II AV-block or acute third } \\
\text { degree block with nodal escape rhythm }\end{array}$ & 1578.34 & 1499.89 & 36 \\
\hline$d / 3$ & acute right bundle branch block & 1502.21 & 1505.35 & II \\
\hline$e / 4$ & $\begin{array}{l}\text { acute third degree block with ventricular } \\
\text { escape rhythm }\end{array}$ & 1133.25 & 627.88 & 11 \\
\hline$f / 5$ & recurrent right bundle branch block & 2367.52 & 2428.02 & 18 \\
\hline$g / 6$ & $\begin{array}{l}\text { third degree block combined with ventricu- } \\
\text { lar escape rhythm in follow-up phase }\end{array}$ & 3623.75 & 2123.74 & 4 \\
\hline
\end{tabular}

$\operatorname{Eta}^{2}: .06(p<.001)$

\section{* Severity of heart failure.}

Variance of CCU costs explained by the severity of heart failure as measured by the Left Ventricular Ejection Fraction (see $\$ 6.2 .8 .1$.) amounts to .15 (table 7.25). Patients with a LVEF between $20 \%$ and $40 \%$ show moderately high correlations with CCU cosis (DLVEF1: $r=.26 ; p<.001$ and DLVEF2: $r=.28 ; p<.001)$. In a dummy regression analysis containing only the three LVEF-variables, two of the three are statistically significant (DLVEF1 and DLVEF2) and the third (DLVEF3) is just non-significant ( $\mathrm{p}=.06)$. When the length of stay and the type of admission and discharge are included in the analysis, all three effects are significant. When all other severity-indicators are added to the regression equation, then only the presence of an ejection fraction between $30 \%$ and $40 \%$ (DLVEF1) will cause a significant rise in CCU costs (table 7.26). Having an ejection fraction between $20 \%$ and $30 \%$ (DLVEF2) or less than $20 \%$ (DLVEF3) will not increase CCU costs at all. This rather puzzling effect may be explained by other severity indicators. In general, patients tend to have a low ejection fraction when they have cardiac wall motion abnormalities in the anteroseptal area. As we have seen above, patients with this abnormality tend to have higher CCU costs. Furthermore, specific rhythm disorders are associated with a low ejection fraction: recurrent sinustachycardia is positively correlated with an ejection fraction lower than $20 \%$ (DRHYTHM5:r=.20;p<.001) and ventricular fluter/-fibrillations or sustained ventricular tachycardia in the latter part of the stay is highly related to an ejection fraction between 20 and $30 \%$ (DRHYTHM9: $r=30 ; p<.001$ ). Both lactors will at the same time cause a sharp rise in CCU costs. Also, a LVEF score between $20 \%$ and $30 \%$ will be related to a longer length of stay (LOS: $r=.30 ; p<.001$ ) which naturally will be associated with a higher chance of incurring CCU costs.

The severity of heart failure measured by the Left Ventricular End Diastolic Dimension (LVEDD) appears to be correlated with CCU costs: patients with deviating LVEDD of more than $55 \mathrm{~mm}$ show a significantly higher chance of incurring CCU costs $(r=16 ; p<.001)$. But in the eventual regression analysis the LVEDD has no significant effect on CCU costs. This may be explained by the fact that patients showing LVEDD-deviations will generally suffer from cardiac wall motion abnormalities in the anteroseptal side of the left ventricle (CWMLOC1:r=.14;p<.001) and this factor will cause substantially high CCU costs. Furthermore there are positive correlations between the LVEF-dummy variables and the LVEDD, for instance the correlation between LVEDD and DLVEF2 is .28 ( $p<.001)$ and that 
with DLVEF3 is .17 ( $p<.001)$. Although the relative effect of these last dummy variables on the CCU costs is not significant, this will help to make the effect of LVEDD in the eventual regression analysis non-significant.

Severity of heart failure located in the right ventricle, measured by the collapse of the Vena Cava Inferior (VCI) index (see $\$ 6.2 .8 .2$.), seems to be somewhat weakly correlated with CCU costs $(r=.17 ; p<.001)$. In addition when patients have cardiac wall motion abnormalities in the right ventricle, chances are that they will have somewhat higher CCU costs (CWMRV: $r=.09 ; p<.05)$. However, in the eventual regression analysis both effects turn out to be non-significant, because patients with limited collapse of the vena cava inferior have higher chances of suffering from cardiac wall motion abnormalities in the anteroseptal side (DCWMLOC1: $r=.16 ; \mathrm{p}<.001)$. They also tend to have a low score on the LVEF index (LVEF2:r=.17; $<<.001$ ), and typical conduction disorders (DCONDUC6:r=.15; $<<.001$ ). They will - perhaps as a result of this - have a prolonged stay in hospital (DHOME3: $\mathrm{r}=16: \mathrm{p}<.001)$. Patients suffering from cardiac wall motion abnormalities in the right ventricle (CWMRV), will generally have supraventricular rhythm disorders and a high level of CPK enzyme release in the blood serum. Right-ventricular infarction measured by STchanges turns out to have no correlation with CCU costs at all (ISRV: $r=.00 ; p=.49$ ).

It may be concluded that severity of left ventricular heart failure, measured by LVEF, has a substantial effect on CCU costs: patients with a LVEF score between $30 \%$ and $40 \%$ will have higher $C C U$ costs. Right-sided heart failure will not lead to an increase in CCU costs.

Table 7.25 Mean CCU costs in patients within severity categories representing the left Ventricular Ejection Fraction $(\mathrm{n}=461)$.

$\begin{array}{llrrr}\text { Category } & \text { Description } & \text { Mean } & \text { Sid.dev. } & \text { Freq. } \\ & & & & \\ 0 & \text { normal }(L V E F>40 \%) & 1091.48 & 775.06 & 183 \\ 1 & \text { slightly impairment }(30 \%<L V E F<40 \%) & 2143.06 & 1842.53 & 79 \\ 2 & \text { severe impaiment }(20 \%<L V E F<30 \%) & 2493.14 & 2172.57 & 50 \\ 3 & \text { very severe impairment }(L V E F<20 \%) & 1811.88 & 1555.38 & 8 \\ & \text { missing } & 762.79 & 522.52 & 141\end{array}$

$E t a^{2}: 15(p<.001)$

\section{* Severity of physical deficiencies and specific cardiac complications.}

Physical deficiencies (see \& 6.2.9.) which are significantly correlated with CCU costs are palpitations (PALP: $r=.15 ; \mathrm{p}<.001)$, precollapse (PRECOL: $\mathrm{r}=.16 ; \mathrm{p}<.001$ ), syncope/collapse (SYCOL:r=.08:p<.05), dyspnea (DYSPN:r=.15:p<.01), low blood pressure (LBLPRES:r=.14;p<.01) and !ung edema (LEDEMA:r=.15;p<.0i). In the eventual regression analysis none of these physical deficiencies turns out to be an additional explanation for the CCU costs:

Patients who have palpitations during their stay are more likely to have atrial flutter/fibrillations or sinustachycardia in the first part of their stay combined with ventricular flutter/-fibrillations or sustained ventricular tachycardia later on in their stay (DRHYTHM9: $\mathrm{r}=.22 ; \mathrm{p}<.001$ ). In addition to this, these patients have a greater chance of suffering from pericarditis (PERIC: $r=13: p<.01$ ). Both confounding factors explain the assumed effect of palpitations on the CCU costs. 
Patients suffering from pre-collapse during their stay will also have higher chances of having atrial flutter/-fibrillations or sinustachycardia in the first part of their stay followed by ventricular flutter/-fibrillations or sustained ventricular tachycardia later on (DRHYTHM9:r=.19;p<.001). At the same time their length of stay is positively correlated with pre-collapse during stay (LOS: $r=.24 ; p<.001$ ) and their hospital discharge tends to be rather late (DHOME3:r=13; $<.001$ ). All three factors will explain any presumed effect of pre-collapse on the CCU costs.

Patients who have a syncope/collapse during their stay are more likely to suffer from anteroseptally located cardiac wall motion abnormalities (DCWMLOCl: $r=.20 ; \mathrm{p}<.001$ ) and will have a longer stay in hospital (LOS: $r=.19 ; \mathrm{p}<.001)$. At the same time these patients tend to be discharged rather late (DHOME3: $r=13: \mathrm{p}<.01)$.

Patients who have lung edema during their stay are likely to suffer from anteroseptally located cardiac wall motion abnormalities (DCWMLOCl:r=.25;p<.001) and at the same time are likely to have atrial flutter/-fibrillations or sinustachycardia in the first part of their stay followed by ventricular flutter/-fibrillations in the latter part of their stay (DRHYTHM9:r=20;p<.001). Also, these patients suffer from recurrent sinustachycardia during their stay (DRHYTHM5: $\mathrm{r}=.15 ; \mathrm{p}<.001$ ). Finally. they tend to have low a Left Ventricular Ejection Fraction, between $20 \%$ and $30 \%$ (DLVEF2:r=.32;p<.(O)1). All factors mentioned above will explain any assumed effect between lung edema and CCU costs. All other physical deficiencies caused by AMl hitherto not mentioned have no significant effect on the CCU costs.

Specific cardiac complications highly correlated with CCU costs are pericarditis (PERIC: $\mathrm{r}=.34 ; \mathrm{p}<.001)$ and mitral insufficiency (MITINS: $\mathrm{r}=11 ; \mathrm{p}<.05)$. Only pericarditis has a significant increasing effect on the CCU costs in the eventual regression analysis (table 7.26). Patients having mitral insufficiency are likely to stay longer in hospital (LOS: $r=.20 ; p<.001$ ). At the same time they tend to be in the highest category of the total CPK-release (DCPK5: $r=.22 ; p<.001$ ). Other complications like cardiogenic shock (CSHOCK:r $=-.05 ; p=.13$ ) and CVA/TIA (CVA: $r=-.03 ; p=.27$ ) show no significant correlations with CCU costs at all and turn out to have no effect on the CCU costs.

It may be concluded that only one specific cardiac complication related to AMI has a substantial effect on the CCU costs: patients with pericarditis will incur higher CCU costs.

\section{* Characteristics of admission and discharge, and length of hospital stay.}

From the results from regression analysis (table 7.26) it may be concluded that in addition to the patient's severity of illness, conditional factors like type of admission and type of discharge are also significantly related to the CCU costs. Patients admilted via other hospitals ten days after having stayed in the previous hospital have significantly lower CCU costs. If patients are discharged in $\mathrm{T} 3$ or, if patients are transferred to other medical departments in T3, CCU costs tend to be relatively high. If patients die within ten days their CCU costs are relatively lower.

\section{* Background characteristics.}

None of the patient's individual background characteristics (see $\$ 6.3 .1$ ) show significant comelations with the CCU costs. In the eventual regression analysis each presumed effect involving background characteristics is not statistically significant. In almost all cases the background variables are highly correlated with one or more indicators measuring severity of illness.

It may be concluded that individual background characteristics of patients will play no role in the explanation of $C C U$ costs at all. 
Table 7.26 Results from the eventual regression analysis: dependent variable is $\mathrm{CCU}$ costs for AMI patients (Listwise deletion of missing cases, $n=459$ ).

\begin{tabular}{|c|c|c|c|c|}
\hline Independent Variables & $B$ & $S E B$ & Beta & $\operatorname{Sig} T$ \\
\hline ISLOC & 316.70 & 76.01 & .14 & .000 \\
\hline$D C P K$ & -23.87 & 317.96 & .00 & NS \\
\hline$D C P K I$ & 50.32 & 316.08 & .01 & NS \\
\hline DCPK2 & 186.79 & 320.58 & .05 & NS \\
\hline$D C P K 3$ & 285.77 & 316.36 & .09 & NS \\
\hline$D C P K 4$ & 661.82 & 324.38 & .17 & .042 \\
\hline$D C P K 5$ & 1161.36 & 328.07 & .28 & .000 \\
\hline DECHO & $=98.58$ & 109.36 & .03 & NS \\
\hline DCWMMIS & 164.05 & 336.97 & .01 & NS \\
\hline DCWMLOCI & 350.77 & 118.71 & .12 & .003 \\
\hline DRHYTHMI & 203.46 & 102.90 & .06 & NS \\
\hline DRHYTHM2 & -139.67 & 201.33 &. .02 & NS \\
\hline DRHYTHM3 & -125.19 & 218.99 & .02 & NS \\
\hline DRHYTHMA & 738.69 & 211.72 & .11 & .005 \\
\hline DRHYTHMS & 1018.30 & 187.23 & .17 & .000 \\
\hline DRHYTHMO & 376.54 & 356.53 & .03 & NS \\
\hline DRHYTHM7 & 89.27 & 174.34 & .02 & NS \\
\hline DRHYTHMS & 1411.08 & 343.44 & .13 & .000 \\
\hline DRHYTHM & 3467.50 & 364.86 & .31 & .000 \\
\hline ICONDUCI & -160.95 & 260.79 &. .02 & NS \\
\hline DCONDUC2 & 114.54 & 151.68 & .02 & NS \\
\hline DCONDUC3 & 199.90 & 271.38 & .02 & NS \\
\hline DCONIUCA & -123.21 & 287.50 & -.01 & NS \\
\hline ICONDUCS & 537.77 & 215.78 & .08 & .013 \\
\hline DCONDUC6 & 202.51 & 4.4 .44 & .01 & NS \\
\hline DLVMIS & 391.17 & 943.02 & .01 & NS \\
\hline DLVEFI & 357.79 & 125.31 & .10 & .005 \\
\hline DLVEF2 & 123.50 & 165.42 & .03 & NS \\
\hline DLVEF3 & 187.98 & 376.76 & .02 & NS \\
\hline PERIC & 404.62 & 149.53 & .09 & .007 \\
\hline LOS & 2.11 & 6.16 & .01 & NS \\
\hline DADINTO & -377.06 & 241.75 & .05 & NS \\
\hline IDADEXTI & 9.69 & 370.65 & .00 & NS \\
\hline DADEXT2 & -554.57 & 321.65 & -.05 & NS \\
\hline DADEXT3 & -1486.23 & 504.02 & -.09 & .003 \\
\hline DHOMEI & -124.10 & 359.27 & .01 & NS \\
\hline DHOME3 & 710.23 & 105.65 & .25 & .000 \\
\hline DTRANSI & -333.45 & 496.17 & .02 & NS \\
\hline DTRANS2 & -437.31 & 499.53 & -.03 & NS \\
\hline DTRANS3 & 3342.88 & 846.01 & .12 & .000 \\
\hline DDEATHO & -313.13 & 347.34 & -.03 & NS \\
\hline DDEATHI & -508.66 & 237.36 & -.08 & .033 \\
\hline DDEATH2 & .537 .14 & 264.34 & .07 & .043 \\
\hline DDEATH3 & -55.92 & 366.40 & .00 & NS \\
\hline CONSTANT & 31.84 & 182.72 & & NS \\
\hline \multicolumn{5}{|c|}{$\begin{array}{l}\text { Explained Variance }\left(R^{*}\right): .67 \\
B=\text { unstandardized (partial) regression coefficient; SE } B=\text { standard error belonging to } B ; B E T A= \\
\text { standardized (partial) regression coefficient: } S I G T=\text { significance of } B \text { or BETA according to the } T \text {. } \\
\text { distribution (l degree of freedom) }\end{array}$} \\
\hline
\end{tabular}




\section{Discussion.}

It may concluded that the severity of illness of patients with AMI has an important increasing effect on CCU costs. Severity indicators explaining an increase in CCU costs are (1) anteroseptally located ischemia measured by ECG at rest, (2) very high enzyme release, (3) anteroseptally located cardiac wall motion abnormalities,(4) specific cardiac rhythm disorders, (5) specific cardiac conduction disorders, (6) a LVEF between $30 \%$ and $40 \%$ and (7) pericarditis. Variance explained by these severity indicators alone is $58.1 \%$.

In almost all cases the presumed effects of other severity indicators having significant correlations with the CCU costs but having non-significant effects in the eventual regression. analysis turn out to be explained by the correlations with one or more of the already mentioned deficiencies. Some of these severity indicators turn out to be also significantly correlated with the length of stay. Because of this their presumed effects on CCU cosis turn out to be non-significant in the eventual regression analysis. Introducing the length of stay as a conditional variable next to the severity indicators does increase the variance which can be explained to $59.0 \%$. Inclusion of the "death' dummy variables next to the severity of iliness indicators increases the variance explained to $60.1 \%$. Death within ten days of the stay is negatively associated with CCU costs.

Next to the severity of illness indicators, the variance explained by the type of admission and transfer turns out to be low (total is $58.5 \%$ resp. $59.3 \%$ ). However, the beta parameters are significant in the eventual regression analysis. One may conclude that patients coming from other hospitals will generally be admitted to the routine nursing unit and not to the CCU. Patients transferred to another medical department in the same hospital will have higher CCU costs, probably due to specific complications or co-morbidities. Other types of non-regular discharge (DHOME1 and DHOME3) in addition to the severity indicators, will increase the additional explained variance, to $63.5 \%$. All the presumed effects of severity indicators mentioned do stay significant in the eventual regression analysis when the conditional variables are introduced. When the DHOME-variables are introduced in the last step in regression analysis some regression coefficients of scverity indicators such as DRHYTHM8 (first $B=1693.5$ now $B=1434.1$ ) and DCONDUC6 (first $B=397.6$ now 200.87) turn out to decrease. All other significant effects of indicators show only a minor decrease in the regression coefficients. Similar to the previous analysis there is a preferrence for the solution in which the regression coefficients are subjected to strict control by confounding variables which may cause some underestimation of the effects. In total $67 \%$ of the variance in CCU costs is predicted by the severity of illness and conditional factors. The relative importance of these variables for the CCU costs (beta-parameters) is presented in diagram 7.27 . 
Diagram 7.27 Path analytic model in which an explanation is given of the CCU costs for patients having AMI (Listwise deletion of missing cases, $n=459$ ).

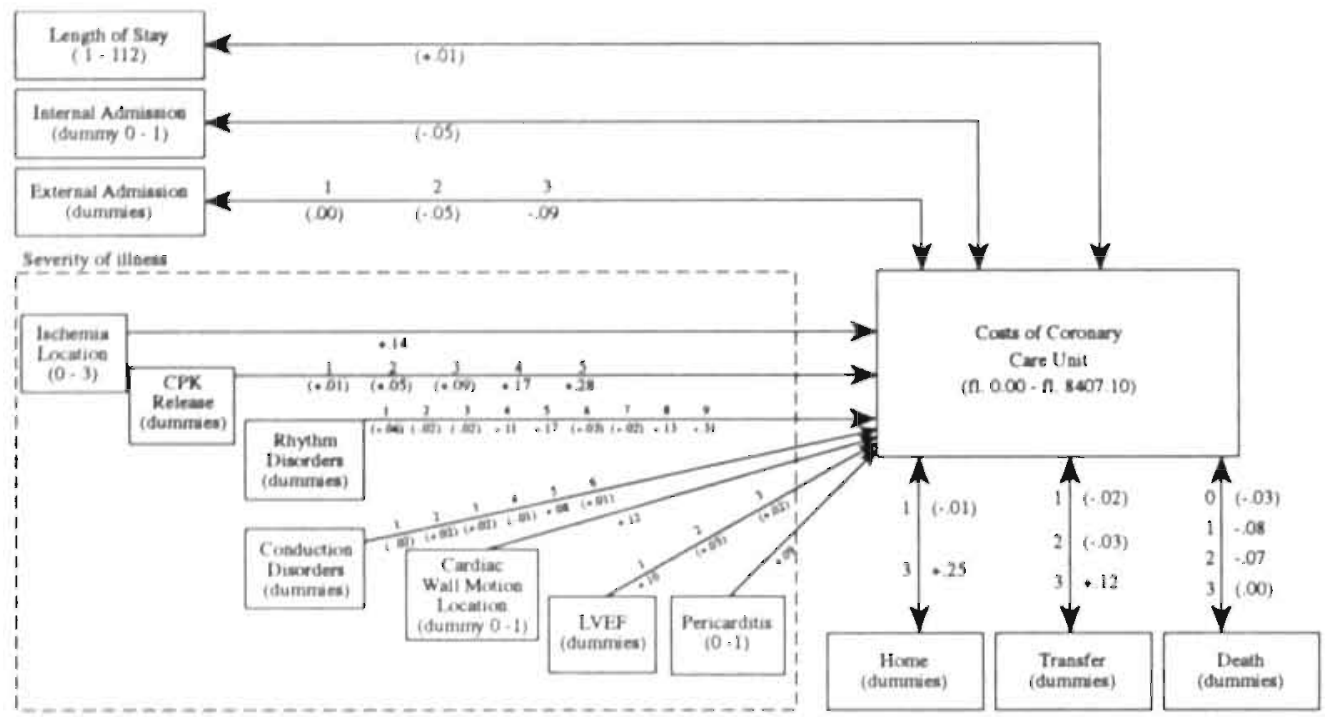

lexplained Variance $\left(\mathbb{R}^{2}\right)$; $67^{\circ}$

Effects of dummy variables in breakets are not significant at the $5 \%$ level. Only beta parameters are shown.

\subsubsection{Explanation of other costs of medical care.}

Relations between costs of drugs, costs of laboratory services, costs of ECG, costs of echocardiography and costs of exercise tests with severity indicators are analyzed in the same way as length of stay and $\bar{C} C U$ costs. For reasons of shortness only the results of each analysis will be summarized here. Correlation matrices and results from regression analysis will be presented in the appendices.

\section{* Explanation of costs of drugs.}

Severity indicators which have a substantial effect on the costs of drugs according to the results in appendix 27 are: (1) severity of ischemic pain; patients still having ischemic pain after three days incur higher costs for dnugs, (2) severity of rhythm disorders; patients with acute sinustachycardia of atrial flutterflibrillations combined with ventricular flutter/fibrillations or sustained ventricular tachycardia later on have higher costs for drugs, (3) severity of hear failure measured by Left Ventricular End Diastolic Dimension (LVEDD); patients with LVEDD of more than $55 \mathrm{~mm}$ have higher costs for drugs, and (4) one physical defieiency caused by AMI; patients with edema located in ankles or feet will have higher costs for drugs. All other severity indicators turn out to have no substantial effect on the costs of drugs. In almost all cases severity indicators as well as costs of drugs (appendices 22-26) appear to be highly associated with the length of stay. When length of stay of patients is introduced in regression analysis presumed effects of a number of severity indicators turn out not to be significant anymore. Costs of drugs have a very high positive correlation with the patient's length of stay $(r=78 ; p<001)$. One could say that - besides some functional medical deficiencies directly or indirectly caused by myocardial necrosis - the costs of drugs can be explained on a day-fo-day basis. The severity of illness may be seen as an indirect. faclor for costs of drugs; because more severe patients will stay in hospital Ionger their costs of drugs will rise accordingly. Furthermore, next to the patient's severity of illness and. 
length of stay, conditional factors like type of admission and type of discharge are in some cases significantly related to costs of drugs: patients who get an infarction while in hospital have lower costs of drugs, and if patients are transferred to other medical departments during the acute or post-acute phase of their stay, their costs for drugs will also be lower. Individual background characteristics of patients are irrelevant in the prediction of costs of drugs. In total $75 \%$ of the variance in costs of drugs is predicted by both severity of illness and these conditional factors.

\section{* Explanation of costs of laboratory services.}

Severity indicators having a substantial effect on costs of laboratory services delivered to AMI-patients are (appendix 33): (1) the location of myocardial necrosis; patients with anteroseptally located necrosis will have higher costs for laboratory services than other patients, (2) CPK-enzyme release; if the CPK enzyme release is moderately high (between 10.000 and $20.000 \mathrm{U} / 1 *$ hours $)$ or extremely high $\left(>60.000 \mathrm{U} / \mathbf{H}^{*}\right.$ hours) patients will also have higher costs for laboratory services, (3) severity of ischemic pain; patients with ischemic pain only in the beginning as well as patients with prolonged ischemic pain will have higher costs for laboratory services, (4) severity of rhythm disorders; patients with acute ventricular flutter/-fibrillations combined with atrial flutter/-fibrillations or sinustachycardia later on and patients with acute sinustachycardia or atrial flutter/-fibrillations combined with ventricular flutter/-fibrillations or sustained ventricular tachycardia later on will have higher costs for laboratory services, (5) severity of conduction disorders; patients with an acute third degree AV-block. with ventricular escape rhythm will incur higher costs for laboratory services, (6) severity of right sided-heart failure; patients with a collapse of the Vena Cava Inferior of less than $50 \%$ will incur higher costs for laboratory services and (7) one specific complication; patients with a C.VA or TlA during their stay will have higher costs for laboratory services.

All other severity indicators showing (significant) relations with the costs of laboratory services (appendices 28-32) also appear to be highly associated with the length of stay or with one of the severity criteria mentioned above and will therefore produce no substantial effect on these costs. The costs of laboratory services are moderately correlated to the patient's length of stay $(r=.36 ; p<.00 !)$. It may concluded that when patients stay in hospital longer, costs of laboratory services will increase. In addition, patients who get an infarction while in hospital have lower costs for laboratory services. This is also the case if patients die immediately within period TO. Furthermore, when patients are transferred to other medical departments their costs for laboratory services (requested by cardiologists) will naturally be lower. Data analysis also shows that patients attending the PRIMI-protocol will have higher costs for laboratory services, irrespective of their severity of illness, their length of stay and type of admission or discharge. Individual background characteristics of patients turn out to have no additional predictive value. In total $72 \%$ of the variance in costs of laboratory services is predicted by the severity of illness plus conditional factors.

\section{* Explanation of costs of ECG.}

The only severity indicator having a substantial effect on the costs of ECG of AMI-patients turns out be the severity of rhythm disorders (appendix 40); patients with sinustachycardia or non-sustained ventricular tachycardia exclusively in the follow-up phase and patients with acute sinustachycardia or atrial futter/-fibrillations combined with ventricular flutter/. fibrillations or sustained ventricular tachycardia later on will have higher costs for ECG. All other severity criteria having a significant associations with the costs of ECG (appendices 34-39) turn out to have no substantial effect on these costs, when at the same time the conditional factors are introduced in the regression analysis. An extremely high correlation 
is present between the length of stay and costs of ECG $(r=.87 ; p<.001)$, indicating that the longer the patient's hospital stay the higher the costs for ECG. This is not surprising since AMI patients routinely get at least one ECG per hospital day. So the question to explain the cost of ECG may be answered by explaining the patient's length of hospital stay. If patients die or are transferred, then chances of having high costs of ECG will naturally decrease. Individual background characteristics of patients have no predictive power whatsoever on the costs for ECG. In total $89 \%$ of the variance in costs of ECG is predicted by the combination of severity of illness and conditional factors.

\section{* Explanation of costs of echocardiography.}

Severity indicators having a substantial effect on costs of echocardiography are (appendix 44) lung edema and pericarditis; if patients suffer from lung edema or pericarditis costs of echocardiography will rise significantly. Other severity indicators that are significantly associated with these costs (appendices 41-43) turn out to have no substantial effect on costs of echocardiography; in almost all cases these costs also highly correlated with the length of stay at the CCU. The length of stay at the CCU turns out to be the crucial 'in between' factor in the data analysis since this variable is highly correlated with costs of echocardiography $(\mathrm{r}=.48 ; \mathrm{p}<.001)$; costs of echocardiography tend to rise, if patients stay in CCU longer. This is not surprising since specific results of echocardiography are normally used to decide whether or not a patient can leave the CCU. In addition to this, if patients die during stay, get discharged within three days or are transferred to other medical departments within ten days costs will naturally be lower. Again, individual background characteristics of patients do not secm to be relevant in predicting costs of echocardiography. In total $32 \%$ of the variance in costs of echocardiography is predicted by the severity of illness combined with the conditional factors.

\section{* Explanation of costs of exercise tests.}

From regression analysis (appendix 48 ) it may be concluded that the severity of illness has a low but negative effect on the costs of exercise tests (Bruce or thallium). It turns out that if patients have recurrent atrial flutter/fibrillations, or if they have edema in ankles or feet, or if they have a CVA or TIA an exereise test is generally not performed. Other severity indicators significantly associated to costs of exercise tests (appendices $45-47$ ) appear to be either closely related with (one of) the mentioned severity criteria or with (one of) the conditional factors. Patients will normally get an exercise test just before they leave the hospital. Patients coming from other hospitals or being discharged within three days, or deceased patients (the latter may be obvious) have lower chances of getting an exercise test. The same holds for older patients; they turn out to have significantly lower costs for exercise tests, In total $25 \%$ of the variance in costs of exercise tests is explained by the combination of severity of illness and conditional factors. 


\subsection{Conclusions.}

The results of the regression analysis regarding the differences in costs of ten types of specific medical care given to patients with Acute Myocardial Infarction explained by (1) the patient's severity of illness, (2) medical treatment, (3) contra-indications for medical treatment, (4) participation of patients in clinical trials, (5) background characteristics, and (6) conditional factors related to characteristics of the patient's hospital stay will now be summarized.

\section{Severity of illness.}

In general, the influence of the patient's severity of illness on medical care costs tends to show variations in strength: sometimes the effects are relatively large, sometimes no effect is to be found at all. Four degrees in the strength of the relative influence on costs and length of stay may be distinguished (A-D).

\section{A) Severity of illness in AMI-patients has a large effect on their length of stay and their CCU costs.}

Differences in length of stay and CCU costs are not explained by exactly the same severity indicators, although some indicators have an effect on both dependent variables. The patient's length of stay is, for the greater part, explained by the clinical causes and consequences of myocardial necrosis, but not so much by the severity of the necrosis itself. One of the causes of AMI, myocardial ischemia, will increase hospital stay, particularly if it is located in the anteroseptal side of the left ventricle. Consequences of AMi like specific cardiac rhythm disorders, heart failure in the left ventricle, pericarditis, mitral insufficiency and CVA/TIA will each increase the length of stay substantially. Furthermore, (still) experiencing ischemic pain after several days in hospital or having a pre-collapse during stay are additional factors that will prolong hospital stay. The stay in (and costs of) the CCU for patients with AMI, will increase partly as a result of the same factors that also have an effect on length of stay: anteroseptal myocardial ischemia, specific cardiac rhythm disorders, heart failure in the left ventricle and pericarditis. However, other factors, explaining the patients' length of stay have no additional effect on CCU cost. On the other hand high scores on some severity indicators, such as an extremely high enzyme release (CPK), anteroseptally located cardiac wall motion abnormalities and recursent right bundle branch block are relevant and typical for an increase in CCU costs.

\section{B) Severity of illness in AMI-patients has a moderate effect on costs of thrombolytic therapy and costs of laboratory services.}

Whether patients receive thrombolytic therapy is dependent on their acute severity condition on admission: patients with anteroseptally located necrosis (indicated by typical Q-waves in ECG) and extended myocardial ischemia over more wall segments in the left ventricle (indicated by typical ST-changes in ECG) have a higher chance of getling thrombolytic therapy. Furthermore, this chance increases if patients suffer from ischemic pain on admission.

Severity indicators related to myocaidial necrosis have an effect on costs of laboratory services: patients with anteroseptally located necrosis or with a moderately or extremely high enzyme release will generally get more laboratory tests. If patients suffer from ischemic pain there is an additional reason for doing more laboratory tests, probably to check for recurrent infarction. Furthermore, the consequences of myocardial necrosis also have an additional effect on the costs of laboratory services: if patients suffer from specific rhythm 
or conduction disorders, if they show right ventricle failure, or if they get a CVA/TIA, this will certainly lead to more laboratory services. Regardless of these severity indicators, the costs of laboratory services are also highly correlated to the patient's length of stay: if patients stay in hospital longer, costs will naturally rise.

\section{C) Severity of illness in AMI-patients has a relatively weak effect on the costs of acute catheterization, costs of acute PTCA, the total costs of drugs, the costs of ECG and the costs of echocardiography.}

The costs of acute catheterization turn out to be weakly influenced by the patient's severity of illness. Patients with ischemia extended over more wall segments have higher chances of receiving a catheterization. However, the results indicate that whether or not patients receive thrombolytic therapy may offer a better prediction for costs of acute catheterization.

One vessel disease during T0 will increase the costs due to (acute) PTCA. If these patients show occluded coronary stenosis this will certainly enlarge chances of getting a PTCA. Furthermore, if myocardial necrosis is located in the anteroseptal area chances that. PTCA will be performed will somewhat increase.

The total costs of drugs and ECG are strongly related to the patient's length of stay and the way patients are admilled to and discharged from the hospital. These costs will rise progressively with the length of stay. The costs of echocardiography are strongly correlated to the iength of stay at the CCU: if patients tend to stay at the CCU longer the costs of echocardiography will definitely rise. The severity of illness of AMI may be seen as the indirect cause of specific costs. As we have seen in previous data analysis, severely ill patients will stay longer at the hospital (c.q. at the CCU). Because of this they will presumedly have higher costs for drugs, ECG and echocardiography. Thus, severity indicators explaining the length of stay at the hospital or the costs of CCU may be considered indirectly responsible for increasing the costs of drugs, ECG or echocardiography. The severity of illness also has a direct effect on the latter costs. The costs of drugs, for instance, will increase additionally if patients still have ischemic pain after several days in hospital, if they have very severe cardiac rhythm disorders, if they have right-sided heart failure or if they have edema in the ankles or feet. The costs of ECG's will increase additionally if patients have severe cardiac rhythm disorders. The cosis of echocardiography will increase, if patients suffer from lung edema or pericarditis. As a general conclusion one might state that the severity of illness of AMI patients does have indirect as well as direct effects on these specific costs.

\section{D) The severity of illness will not increase costs due to exercise tests.}

In contrast to previous results, specific types of severity of illness will tend to decrease the costs made by exercise tests. If AMI patients have recurrent atrial flutter/-fibrillations, have edema in the ankles, feet or abdomen, or suffer from CVA/TIA during stay, they have lower chances of incuring costs for exercise tests.

\section{Medical treatment.}

Medieal treatment as such wil! sometimes have a strong relation to the costs for other medical care, regardless of the severity of illness. From the data analysis it is found that thrombolytic therapy is highly positively correlated with the costs due to acute heart catheterization: acute thrombolytic therapy appears to go hand in hand with subsequent acule heart catheterization, apparently to ascertain the effects of the treatment. On the other hand, acute thrombolytic therapy is negatively associated with the costs due to PTCA for patients with one vessel discase. Patients having had acute thrombolytic therapy seem to have lower chances of getting a PTCA. 


\section{Contra-indications.}

Obviously, contra-indications will have a restrictive impact on incurring costs of treatment. The only specific contra-indications in this study concern those for thrombolytic therapy. At the cardiology department in our study three major contra-indications for this form of treatment did exist at the time of investigation: having ischemic pain for more than six hours, being in coma, or being older than 75 years. All AMI patients with one of these contra-indications do not incur any costs for thrombolytic therapy at all.

\section{Participation of patients in clinical trials.}

Enrollment in a clinical trial will naturally influence the costs of medical care. In the patient population at the time of investigation this concerned only one specific project (i.c. PRIMItrial), in which 59 patients participated. Enrollment in this clinical trial involved a standard incurrence of costs for thrombolytic therapy, heart catheterization and laboratory services.

\section{Background characteristics.}

A patient's background characteristics turn out to have no predictive value for costs of medical care, if one takes into account the patient's severity of illness on admission or during hospital stay. The only exceptions are the patient's age and sex; older patients will have a lower chance of incurring costs for exercise tests. At the time of investigation, the patient's age (older than 75 years) did form a decisive contra-indication for thrombolytic therapy. It has also been found that female patients have a significantly lower chance to get an acute heart catheterization.

\section{Conditional factors related to characteristics of the patient's hospital stay.}

Although conditional factors have essentially been used in the regression analysis to distinguish real effects from spurious effects between severity of illness and costs, these factors themselves also turn out to have significant predictive power towards costs of medical care. These significant (partial) relations between conditional factors and costs could not be explained by the severity indices used in the data analysis. For instance, deceased patients differ very much in costs compared to non-deceased patients. Furthermore, patients who die very soon during their stay have a shorter length of stay and therefore relatively lower costs in contrast to patients who die after a long time in hospital. Because the actual cause of death of these patients has not been included in this study, the factor 'decease during hospital stay' may be regarded as an important "explanation' for costs.

Other non-regular forms of discharge not explained by the severity of illness turn out to have significant relations with particular costs. An extremely carly discharge will (quite naturally) lead to a very short length of stay and lower costs; circumstances not investigated in this study such as transfer to another hospital might be the reason for this significant (partial) relation. A non-regular late discharge will quite naturally be related to the length of stay and $\mathrm{CCU}$ costs, probably due to factors not captured by our severity indices, such as rare complications or comorbidities. Patients coming from other hospitals turn out to have a shorter length of stay and have lower CCU costs. If patients are transferred to another medical department within the same hospital because of specific complications, their total length of stay will increase. Although these factors will contribute in the prediction of medical care costs they must not be regarded as an explanation for these costs.

The results from the multivariate data analysis as outlined in this chapter will be used to propose a patient classification system for patients having Acute Myocardial Infarction. The methodology of this procedure will be outlined in chapter 8 . 



\section{Classifying acute myocardial infarction patients according to their severity of illness.}

In this chapter a Severity of Illness Classification (henceforth SIC) will be proposed for patients with Acute Myocardial Infarction. First, the empirically based conclusions of this analysis will be compared with the assumptions made by other classification systems used to explain costs of care (8.1.). Next the methodology of the system will be proposed in which patients will be categorized into groups to explain length of hospital stay (8.2.). Then, the predictive validity of the SIC will be compared to the predictive validity of the DRG's (8.3.). Some applications of a SIC will be given (8.4) and finally conclusions of this chapter will be shortly summarized (8.5.)

\subsection{Comparison of severity indicators empirically found in this study and assumptions of other studies.}

All severity indicators found to be relevant in explaining costs of care in AMI-patients have been summarized in table 8.1. If one compares the empirically found severity indicators in this study with the indicators used in other studies (see \$ 2.2) some interesting differences can be observed. The DRG system differentiates AMI patients with and without specific cardiovascular complications. Although some of the severity indicators found to be relevant in this study are included in the complication category (i.e. specific rhythm and conduction disorders and left ventricular heart failure), the DRG-system does not take into account the time at which these deficiencies occur during the patient's hospital stay. Specific illness characteristics such as the location of myocardial ischemia and necrosis, the (time of) appearance of ischemic pain, the total enzyme release and the location of cardiac wall motion abnormalities, which have important effects on the costs, are not specified by the DRG system. Furthermore, the DRG system categorizes patients who died during hospital stay into one separate group. However, one of the conclusions of chapter 7 is that not all patients who die in the hospital have a short slay or low costs.

The same remarks can be made for the Disease Staging system and the PMC system with one exception: both systems place patients with specific complications into separate categories. These systems also do not specify the time of occurrence of these complications nor do they use typical severity indicators obtained from medical history or diagnostic tests.

Disease-specific patient classification systems which place the severily of illness in a more central role (APACHE II, Medisgrps and CSI, see \$2.2.) are much more comparable with regard to the empirical results in chapter 7 . The severity indicators used by the APACHE IIsystem (table 2.7) do not match at all with the severity indicators found in this study. The APACHE II-system is expected to be less suitable for explaining costs of medical care in AMl patients because this system is developed for, and mainly used in, the Intensive Care Units in which patients with various types of iliness may lie. The MEDISGRPS-system uses more specific severity indicators (tabie 2,8). ECG-data that are expected to be relevant for the construction of severity categories in the MEDISGRPS-system are: ischemia/atrial fibrillations, myocardial infaretion and third degree heart block. Following the results in chapter 7. the location of ischemia and necrosis (in the anteroseptal area) turns out to be important. Atrial fibrillations are also found to be: important, certainly if this is followed by 
ventricular fibrillations. Other indicators distinguished by MEDISGRPS such as cardiomegaly, rales, cardiomyopathy, coma and respirations have either not been investigated in this study or turn out to have no direct effect on the costs of medical care. According to MEDISGRPS, heart, failure places a patient in a high severity category, but from our results a distinction needs to be made between left and right sided heart failure. Absolute enzyme values (i.e. CPK) are also used in the MEDISGRPS-system but only relatively high area scores are found to be relevant in our study.

The severity indicators used in the CSI-system (table 2.6) are more akin to the indicators found in our study. Still, the CSI-system makes no distinction in the location of ST-changes. A third degree block and life-threatening arrhythmias are mentioned as relevant indicators. Indicators like cardiac output ( more or less comparable with LVEF and LVEDD) are used. The CSI-system is the only system that uses chest pain as a severity indicator, but it cmphasizes the duration of pain rather than the incidence of pain during stay. In our study, the incidence of ischemic pain is found to be an important indicator. Severity indicators based on physical deficiencies, such as pulmonary edema, cyanosis and dyspnea are also found to be relevant. Other CSI-indicators, such as fever, urine output, white blood counts have not been investigated in this study. The cardiologists consulted did consider these indicators of less importance in measuring the severity of AMI-patients. Specific complications (i.e. pericarditis, mitral insufficiency and CVA/TIA) are distinguished separately: the CSI system applies a weighting rule to measure the overall severity of illness in case of such specific complications or other diseases.

It may be concluded that with regard to AMI patients, large differences exist among the indicators used in patient classification systems. Relevant indicators used in the Computerized Severity of HIness (CSI)-system are somewhat akin to those found in this empirical surdy.

Differences in indicutors used to classify patients are gencrally caused by the differences in the purposes for which a classification system is developed. There are systems which originally are developed to predict utilization of resources and costs (DRG and PMC) and systems which are developed to predict death rates and to analyze quality of care within discase specific categories (Disease Staging, CSI, APACHE II and MEDISGRPS). Differences also exist in the type of criteria used to classity patients: some systems use medical abstract data such as diagnosis and procedures (DRG. PMC and Coded Disease Staging), while others use specific clinical indicators obtained from diagnostic results and the patient's medical history (Clinical Disease Staging. CSI. APACHE II and MEDISGRPS). There are also differences in the methodology used to find criteria for costs differences. There are systems which use criteria derived from a combination of an empirical data analysis and professional judgement (such as DRG and PMC). Most systems are generally only based on the judgement of medical experts (Disease Staging, CSI, APACHE Il and MEDISGRPS). 
Table 8.1 Severity indicators explaining cost of medical care in patients with Acute Myocardial Infarction, which are relevant according to the results of the (dummy) regression analysis reported in chapter 7.

Admission indicators

* coronary stenosis intensity on admission.

* ischemia extent on admission

* necrosis location on admission

* ischemic pain on admission

Dynamic indicators

* ischemia location during stay (ECG at rest)

- necrosis location during stay (ECG at rest)

- CPX enzyme release $(10.000-20.000)$

* CPK enzyme release (40.000-60.000)

* CPK enzyme release ( $>60.000)$

* ischemic pain within 3 days

* prolonged ischemic pain (after 3 days)

* location of cardiac wall motion abnormalities

* acute sinustachycardia

* sinustachycardia or non-sustained ventricular tachycardia excl. in follow-sup phase

* recurrent sinustachycardia or non-sustained ventricular tachycardia

* recurrent artrial flutter/-fibrillations

* acute ventricular flutter/-fibrillations combined with sinustachycardia or non-sustained ventricular tachycardia in follow-up phase

* sinustachycardia or atrial flutter/-fibrillations in the beginning combined with ventricular flutser-fibrillations or sustained ventricular tachycardia in follow-up phase

* acute third degree block with ventricular escape rhythm

* recurrent right bundle branch block

* Left Ventricular Ejection Fraction between $30 \%$ and $40 \%$

* Left Ventricular End Diastolic Dimension $>55 \mathrm{~mm}$

* collapse of Vena Cava Inferior $<50 \%$

* pre-collapse

* lang edema

* body edema

* pericarditis

* mitral insufficiency

* cVartia 


\subsection{Severity of Illness Classification (SIC) for AMI patients.}

Based on the results of chapter 7 one may conclude that three separate classifications of AMI patients are possible: one explaining variations of acute care costs, another for explaining variations in length of stay and thirdly, one for CCU costs. A naive question that may arise is how to develop one overall classification system which will be able to explain each specific type of costs of medical care. Using only the criteria which have an effect on the length of stay (and related costs) will be at the expense of the predictive value of the $\mathrm{CCU}$ costs, and vice versa. The severity on admission may not be a good predictor for the $\mathrm{CCU}$ costs and total length of stay. Another question that may arise, for instance is, if the relevant severity criteria having an effect on the $\mathrm{CCU}$ costs can be substituted by severity criteria having an effect on the length of stay. Therefore, all correlations between the different indicators have been tested for statistical significance. However, we did not succeed in finding adequate substitutions for the different indicators that could also be confirmed from a medical point of view.

So, for the time being priority will be given to develop a classification system based on indicators having an effect on length of stay. This choice has been made because this dependent variable is also often used in other classification systems. Another reason is that overall length of stay is generally assumed to have a relative high correlation with the overall costs of medical care. The total length also includes the length of stay at CCU and it is also correlated to other costs of medical care (drugs, ECG, laboratory services). So, in this chapter priority will be given to the development of only one severity of illness classification: a severity of illness classification explaining length of stay (SIC-LOS). However, the same methodology can be applied if one wishes to explain acute care costs and CCU costs in more detail.

\section{Methodology of a SIC-system.}

In order to classify patients into severity categories, the combination of indicators found to be relevant in regression analysis need to be examined. Relevant severity indicators will be used as severity criteria for a classification of AMI patients. For instance, it seems plausible to statc that patients with anteroseptally located ischemia have a shorter length of stay than patients who, next to this deficiency. also have prolonged ischemic pain after three days. If on top of these deficiencies, patients also have specific cardiac rhythm disorders, hospital stay will be even longer. Thus, the combinations in which the severity criteria occur in patients is crucial to the classification process. In order to develop the SIC-system, the following rules have been applied:

1. The number of categories of patients used in the classification system must be limited, At the same time all categories should have clinical' relevance or be 'clinically valid' regarding their expected duration of stay. This means that the classification is of no (clinical) value, if the cardiologists involved in this study do not individually endorse the classification proposed or do not reach a consensus with regard to the classification. So, the classification is developed in cooperation with an experienced cardiologist.

2. The measurement level of the criteria used in the classification is generally considered as an ordinal scale.

3. Additive procecdings leading to unidimensional scales will be avoided, and more general techniques of non-parametric scale construction will be used. Thus a multidimensional analysis with crosstabulations will be proposed to find the empirical combinations of severity criteria (score patterns). 
4. The categories must be defined as mutually exclusive. If patients have patterns of scores which provide difficulties for classification and at the same time have low or unique frequencies, these patients will be placed in 'adjacent' categories as much as possible. Cases defined as 'non-classifiable' should not exceed a maximum of $5 \%$.

5. Only severity indicators found to be significant in explaining length of stay in regression analysis (see $\S 7.3 .1$.) will be used as criteria in the classification process.

6. Scores for relevant severity criteria will be recoded into a dichotomous (2 classes) or trichotomous ( 3 classes) rank: order using the values of the unstandardized partial regression coefficients (see below). The reason for this reduction is that otherwise the combination of various severity criteria may lead to numerous types of severity categories containing only very small numbers of patients.

7. If patients have missing values for a severity criterion they will get the label (or score) belonging to the category representing the lowest severity for that criterion. In doing so all patients can be classified.

8. Only score patterns having a relevant number of patients will form a separate severity category. The minimum number per category will be set at $1.5 \%$ of the patient population (this is an arbitrary choice).

9. Deceased patients will form two additional categories; score-patterns are assumed to be not relevant for these patients. This will be done because deceased patients differ very much in length of stay and costs compared to non-deceased patients.

Severity criteria relevant for the SIC-LOS classification will be described and a redefinition of scores for these criteria will be presented according to rule 6.

\section{The location of myocardial ischemia measured by ECG at rest.}

Four combined severity categories describing the location of myocardial ischemia have been distinguished (sce table 6.7). The partial regression coefficient for this indicator is +.70 (see table 7.19). Patients with no ST-changes or disappearing ST-changes are -if no other deficiencies are present- expected to stay 6.7 days, which constitutes the intercept value of the regression function (obscrved: $m=8.8$; std.dev. $=2.9 ; n=23$ ). Thus, the length of stay for patients with non-anteroseptal ST-changes (observed: $m=9.7$; std.dev. $=4.7 ; n=277$ ) is expected to increase with .70 day. The length of stay for patients with anteroseptal STchanges (observed: $m=12.4 ; \mathrm{std}$ dev $=7.7 ; n=148$ ) is expected to rise with 1.4 days and for patients with ischemia extending to the anteroseptal wall segment this will be 2.1 days (observed: $m=15.9$; std.dev $=4.7 ; n=7$ ). According to rulc 6 , a new distinction will be made between $(0)$ patients having no ST-changes or having ST-changes corresponding with the non-anteroseptal wall segment and patients with ST-changes corresponding with the anteroseptal wall segment ( 1 ). This recoded criterion will be used in the pattem analysis for the construction of a severity classification.

\section{Ischemic pain.}

Three classes in the severity of ischemic pain have been distinguished (sec table 6.24). Only prolonged ischemic pain after three days in hospital turned out to produce a significant increase in length of stay $(B=+2.4$. see table 7.19). Patients without ischemic pain (observed: $\mathrm{m}=7.7$; std.dev. $=10.0 ; \mathrm{n}=54$ ) or patients with ischemic pain in the first three days of hospital admission (observed: $m=10.0$; std.dev. $=4.9 ; n=306$ ) are expected to have no substantial increase in length of stay. So, the expected length of stay for patients with prolonged ischemic pain after three days in hospital (observed: $m=14.68$; std.dev. $=6.8$; 
$\mathrm{n}=91$ ) will increase with 2.4 days. Therefore, a distinction will be made between $(0)$ patients without ischemic pain or with ischemic pain restricted to the first three days in hospital and (1) patients with prolonged ischemic pain still present after three days in hospital. This recoded criterion will be used in the pattern analysis for the construction of a severity classification.

\section{Cardiac rhythm disorders.}

Ten types of cardiac: rhythm disorders have been distinguished (see table 6.29). Only five types of rhythm disorders turn out to produce a significant increase in length of stay (see table 7.19):

1: acute sinustachycardia (observed; $m=10.16$; std.dev $=4.9 ; n=105 ; B=+1.1$ );

2: recurrent sinustachycardia or non-sustained ventricular tachycardia (observed: $\mathrm{m}=14.9 ;$ std.dev $=8.9 ; n=26 ; B=+2.4)$;

3. recursent atrial flutter/-fibrillations (observed: $m=15.33$; std.dev. $=8.2 ; n=31 ; B=+3.2$ );

4. acute ventricular flutter/-fibrillations combined with atrial flutter/-fibrillations or sinustachycardia in follow-up phase (observed: $m=13.0$; std.dev. $=7.2 ; n=7 ; B=2.5$ );

5. acute sinustachycardia or atrial flutter/-fibrillations combined with ventricular flutter/fibrillations or sustained ventricular tachycardia in the follow-up phase (observed: $\mathrm{m}=23.7$; std.dev, $=4.2 ; \mathrm{n}=9 ; \mathrm{B}=+5.0)$.

After consulting our cariologists and given the values of the partial regression coefficients a distinction is made into three classes: (0) patients who have no cardiac rhythm disorder at all or who have cardiac rhythm disorders with no significant effects on the length of stay according to the results in chapter 7, (1) patients with acute sinustachycardia and (2) patients who hase the other cardic thytho disorders monicaned above. This reculed criterion wiw be used in the patiern analysis for the construction of a severity classification.

\section{Left Ventricular Ejection Fraction.}

The Left Ventricular Ejection Fraction score was previously combined into four classes (see table 6.32 .). The LVEF-score lying between $30 \%$ and $40 \%$ has a significant effect on the length of stay of patients (see table $7.19 ; \mathrm{B}=+1.4$ ), a $L V E F$-score higher than $40 \%$ or lower than $30 \%$ will not lead to a substantial increase in length of stay (see table 7.19). Only patients with a LVEF-score between $30 \%$ and $40 \%$ (observed; $m=13.77$; std.dev $=6.1$; $\mathrm{n}=81$ ) show an additional increase in hospital longer. Therefore, a distinction will be made between (0) patients with a LVEF-score higher than $40 \%$ or patients with LVEF-score lower than $30 \%$. and (1) patients with a LVEF-seore between $30 \%$ and $40 \%$. This recoded criterion will be used in the pattern analysis for the construction of a severity classification

\section{Specific complications and physical deficiencies.}

Specific complications related to AMI and leading to a significant increase in length of stay are (see table 7,19) :

1. pre-collapse (observed: $m=15.16 ;$ std.dev. $=8.2 ; n=32 ; B=+1.4$ );

2. mitral insufficiency (observed: $m=13.7$; std.dev $=9.2 ; n=56 ; B=+1.1$ );

3. pericarditis (observed: $m=15.7 ;$ std.dev $=8.5 ; n=42 ; B=+2.3$ );

4. CVA/TYA (observed: $m=19.3 ;$ std.dev. $=15.8 ; n=8 ; B=+3.6$ ).

One new complication criterion is constructed; a distinction will be made between patients with none of these complications during stay $(0)$, patients with pre-collapse or mitral insufficiency (the length of stay will approximately increase with 1 day); henceforth type 'I" complications and patients with either pericarditis, or CVA/TIA, or both pre-collapse combined with mitral insufficiency; henceforth type ' 2 " complications. The expected length. of stay in the last instance will approximately increase with 2 or 3 days. This recoded criterion will be used in the pattern analysis for the construction of a severity classification. 


\section{SurvivalDecease during stay.}

Although the death of a patient during hospital stay can be seen as a consequence of severity of illness, the analysis has shown that it is an important factor in explaining differences in length of stay of AMI patients. Patients who die during their hospital stay have a different length of stay from patients who do not. Furthermore, a distinction must be made between patients who die early during stay and patients who die from complications after a prolonged spell of illness. A distinction will therefore be made between: $(0)$ surviving patients, (1) patients who die within ten days (observed in period $T 0: m=1.7 ;$ std.dev $=1.9 ; n=10 ; B=-6.3$, in T1: $m=2.4$; std.dev $=.8 ; n=19 ; B=-5.9$, in $T 2: m=6.1 ;$ std.dev. $=2.4 ; n=14 ; B=-4.4)$ and (2) patients who die after ten days in hospital (observed: $m=24.0 ;$ std.dev. $=6.0 ; n=7 ; B=+11.4$ ). This recoded criterion will be used in the pattern analysis for the construction of a severity classification.

\section{Pattern analysis.}

A pattern analysis even with the six recoded severity criteria described above still turns out to lead to numerous severity categories. Theoretically 74 different patterns (including the two 'deceased' categories) may occur (i.e.; in the order of the criteria mentioned: $2 * 2 * 3 * 2 * 3+2=74) ; 59$ of these patterns are empirically observed in the population in question. To reduce the number of severity patterns, a final pairing of criteria is proposed.

Firstly, the criteria location of ischemia and ischemic pain will be combined into one new criteron; from a clinical point of view both severity criteria are representatives of the impaired flow in the coronary artery system.

Anteroseptally located ischemia and prolonged ischemic pain after three days will each lead to an approximate increase in length of stay of nearly two days. Clinically speaking the criteria can be regarded as closely related to each other, so our assumption will be that the weights of the criteria are comparable. If patients have no anteroseptal ischemia and have no prolonged ischemic pain both severity criteria will be used to place a patient into the lowest category. If patients have only anteroseptal ischemia or only prolonged ischemic pain they are placed into the middle category. If patients have both anteroseptal ischemia and prolonged ischemic pain they are placed into the highest category.

Secondly, the relevant cardiac rhythm disorders and the LVEF-critcrion will be combined into one new criterion; both represent functional deficiencies as a consequence of myocardial ischemia and/or necrosis, and may as such be regarded as closely related to each other.

Acute sinustachycardia and a LVEF-score between $30 \%$ and $40 \%$ will each lead 10 an increase in length of stay of approximately one day. If patients have neither rhythm disorders nor a LVEF-score between $30 \%$ and $40 \%$, both severity criteria will be used to place a patient in the lowest category. If patients have either acute sinustachycardia or a LVEF-score between $30 \%$ and $40 \%$, they will be placed into the next category. If patients have either severe rhythm disorders (see point 3 , rhythm category ' 2 ') or acute sinustachycardia combined with a LVEF-score between $30 \%$ and $40 \%$, they will be placed in the next highest category. The highest severity category consists of patients having severe rhythm disorders combined with a LVEF-score between $30 \%$ and $40 \%$. 
The remaining four severity criteria (Location Ischemia/Ischemic pain, Rhythm disorders/LVEF, Complications and Survival/Death) can theoretically be combined into 38 different patterns (including two "deceased' categories i.e. in the order of the criteria mentioned: $3 * 4 * 3+2=38$ ). Empirically 37 patterns can actually be found in our patient population. The observed patterns will be used to construct a severity classification by applying the nine methodological rules described earlier.

Eventually, 17 severity categories are distinguished (see for the results table 8.2., and for an exhaustive description of each severity pattern see appendix 49). The categories in this classification will described below.

Severity category ' 1 ' includes patients who survive, who have no anteroseptally located ischemia, no prolonged ischemic pain, no relevant cardiac rhythm disorders, no relevant LVEF-score and no relevant complications $(\mathrm{n}=108)$.

All other severity categories include AMI patients with deviations from this lowest severity category: The severity categories. will be described below by giving the deviations from this: lowest severity category. Category ' 2 ' to ' 15 ' include patients who survive, category ' 16 ' and ' 17 ' include the patients who die during stay.

Severity category ' 2 " includes patients :

(a) with either anteroseptally located myocardial ischemia $(\mathrm{n}=4 \mathrm{1})$, or

(b) with prolonged ischemic pain $(\mathrm{n}=17)$.

In total 58 patients have been grouped in this category.

Severity category ' 3 ' includes patients with a single complication of type '1' $(n=12)$.

Severity category ' 4 " includes patients:

(a) with either acute sinustachycardia $(n=42)$, or

(b) with a LVEF-seore between $30 \%$ and $40 \%(n=14)$,

In addition, a rest category of 7 patients has been included who have type 'I' complications on top of the above deficiencies.

In total 63 patients are grouped in this category.

Severity category ' 5 ' includes patients with anteroseptally located myocardial ischemia combined with prolonged ischernic pain $(n=13)$.

Severity category ' 6 ' includes patients:

(a) with acute sinustachycardia combined with a LVEF-score between $30 \%$ and $40 \%$ $(n=5)$, or

(b) with severe rhythm disorders $(n=8)$, or

(c) with severe rhythm disorders combined with a LVEF-score between $30 \%$ and $40 \%$ $(n=2)$.

In total is patients are grouped in this category.

Severity category ' 7 ' includes patients with solely a complication of type '2' $(n=12)$.

Severity category ' 8 ' includes patients:

(a) with anterosepta!ly located myocardial ischemia combined with acute sinustachycardia $(\mathrm{n}=10)$, or

(b) with anteroseptally located myocardial ischemia combined with a LVEF-score between $30 \%$ and $40 \%(n=13)$, or 
(c) with prolonged ischemic pain combined with acute sinustachycardia $(n=6)$, or

(d) with prolonged ischemic pain combined with a LVEF-score between $30 \%$ and $40 \%$ $(\mathrm{n}=5)$.

In addition, a rest category of 4 patients has been included who have a type 'I' complication on top of the above combinations of criteria.

In total 38 patients are grouped in this category.

Severity category ' 9 ' includes patients with anteroseptally located myocardial ischemia combined with prolonged ischemic pain, combined with either acute sinustachycardia $(n=6)$, or a LVEF-score between $30 \%$ and $40 \%(\mathrm{n}=2)$.. In addition, 1 patient has been included who has a type ' 1 ' complication on top of the above deficiencies. In total 9 patients are grouped in this category.

Severity category ' 10 ' includes:

(a) patients with anteroseptally located myocardial ischemial combined with either: (1) acute sinustachycardia combined with a LVEF-score between $30 \%$ and $40 \% \quad(n=5)$, or: (2) severe cardiac rhythm disorders $(n=5)$ or: (3) severe cardiac rhythm disorders combined with a LVEF-score between $30 \%$ and $40 \%(n=1)$; or

(b) patients with prolonged ischemic pain combined with either: (1) acute sinustachycardia combined with a LVEF-score between $30 \%$ and $40 \%(\mathrm{n}=0)$ or : (2) severe cardiac rhythm disorders $(n=3)$, or: (3), severe cardiac rhythm disorders combined with a LVEF-score between $30 \%$ and $40 \%(n=1)$.

In total 15 patients are grouped in this category.

Severity category ' 11 ' includes:

(a) patients with acute sinustachycardia, a LVEF-score between $30 \%$ and $40 \%$, and a lype ' 1 ' complication $(n=2)$; or

(b) patients with a type 'l' complication combined with either: (1) severe cardiac rhythm disorders $(n=8)$, or: (2) severe cardiac rhythm disorders combined with a LVEF-score between $30 \%$ and $40 \%(\mathrm{n}=0)$.

In total 10 patients are grouped in this category.

Severity group ' 12 ' includes patients:

(a) with a type ' 2 ' complication combined with acute sinustachycardia and/or a LVEFscore between $30 \%$ and $40 \%(n=4)$; or

(b) with a type ' 2 ' complication combined with severe cardiac rhythm disorders ( $\mathrm{n}=2)$; or

(c) with a type '2' complication and severe cardiac rhythm disorders combined with a LVEF-score between $30 \%$ and $40 \%(n=2)$.

In total 8 patients are grouped in this category.

Severity category ' 13 ' includes:

(a) patients with anteroseptally located myocardial ischemia and a type ' 1 ' complication combined with: either acute: sinustachycardia and a LVEF-score between $30 \%$ and 40 $\%(n=2)$ or: with severe cardiac rhythm disorders $(n=3)$, or: (3) with severe cardiac rhythm disorders combined with a LVEF-score between $30 \%$ and $40 \%(\mathrm{n}=0)$; or

(b) patients with prolonged ischemic pain and with a type ' 1 ' complication combined with either: acute sinustachycardia and a LVEF-score between $30 \%$ and $40 \%(n=0)$, or: with severe cardiac rhythm disorders $(n=2)$, or: severe cardiac rhythm disorders and a LVEF-score between $30 \%$ and $40 \%(n=2)$.

In total 9 patients are grouped in this category. 
Severity category ' 14 ' includes:

(a) patients with anteroseptally located myocardial ischemia and prolonged ischemic pain combined with a type ' 1 ' complication, and with: either acute sinustachycardia combined with a LVEF-score between $30 \%$ and $40 \%(n=0)$, or: with severe cardiac rhythm disorders $(n=1)$, or: severe cardiac rhythm disorders combined with a LVEFscore between $30 \%$ and $40 \%(n=1)$; or

(b) patients with anteroseptally located myocardial ischemia and prolonged ischemic pain combined with a type ' 2 ' complication and with: either acute sinustachycardia combined with a LVEF-score between $30 \%$ and $40 \%(n=0)$, or: with severe cardiac rhythm disorders $(n=4)$, or: severe cardiac rhythm disorders combined a LVEF-score between $30 \%$ and $40 \%(n=1)$.

In total 7 patients are grouped in this category.

Severity category ' 15 ' includes:

(a) patients with anteroseptally located myocardial ischemia combined with a type ' 2 ' complication and with; either acute myocardial sinustachycardia and/or a LVEF-score between $30 \%$ and $40 \%(n=3)$, or: severe cardiac rhythm disorders $(n=3)$, or: severe cardiac rhythm disorders combined with a LVEF-score between $30 \%$ and $40 \%(n=0)$; or

(b) patienis with prolonged ischemic pain combined with a type '2' complication with: either acute sinustachycardia and/or a LVEF-score between $30 \%$ and $40 \% \quad(n=1)$, or: with severe cardiac rhythm disorders $(n=1)$, or: severe cardiac rhythm disorders combined with a LVEF-score between $30 \%$ and $40 \%(n=3)$.

In total 11 patients are grouped in this category.

Severity group '16' includes patients who die in hospital during the acute and post-acute phase of their stay, i.e. periods To to T2 $(n=43)$.

Severity group '17' includes patients who die in hospital during period T3 $(n=7)$.

In total 438 patients have been now classified in one of these 17 nominal severity categories. lligher category numbers do not stand for an linear increase in severity of illness, but in general the measurement level of SIC-LOS will lie between the nominal and ordinal scale. A remaining gfoup of 19 patients $(4.2 . \%)$ can not be classified because of their atypical pattems in severity; logether they form a rest category (category ' 18 ').

The age distribution in the severity categories (appendix 50) varies considerable. Patients in SIC-LOS $\%$ are in average 60.1 . years compared to an average age of 80.6 years in category ' 17 '. Also the distribution of female and male patients in some severity categories tends to vary considerably. For instance $27.6 \%$ of the men are grouped into the lowest SICLOS category ' 1 ' compared to only $17.5 \%$ of the women. In some severity categories large differences in age exist between male and fernale patienis. For instance in categories $2,4,8,9$, and 11 (appendix 50) the average age of women is about six years higher than that of men. Women with AMI appear to be relatively more severe but this must also be seen in relation with the fact that they are relatively older compared to the men with AMI. 
Table 8.2 Severity of Illness, Classification of AMII patients concerning Length of Stay (SICLOS), $n=457$.

\section{SIC Location myocandial ischemial \\ cat. Ischemic pain \\ I Location ischemia (0) and Ischemic pain (0)}

2 Location ischemia (1) or Ischemic pain (l)

3 Location ischemia $(0)$ and Ischemic pain (O)

$4 \quad$ Location ischemia $(0)$ and Ischemic Pain (0)

$5 \quad$ Location ischemia ( $(1)$ and Ischemic pain (I)

6 Location ischemia $(0)$ and Ischemic pain (0)

$7 \quad$ Location ischemia (0) and Ischemic pain (O)

8 Location ischemia (1) or Ischemic pain (I)

$9 \quad$ Location ischemia (1) and Ischemic pain (I)

10 Location ischemia (I) or Ischemic pain (I)

$11 \quad$ Location ischemia $(0)$ and Ischemic pain (0)

I2 Location ischemia (0) and Ischemic pain (0)
Candiac rhythm disor-

ders/LVEF-score

Complication

Frequency

Rhythm disonders ( $O$ ) Complications ( 0 ) 108 and LVEF (O)

Rhythm disonders $(0) \quad$ Complications $(0)$ and $L V E F(O)$

Rhythm disonders (0) Complications (1) and LVEF $(O)$

Rhythm disonders (I) Complications or $\operatorname{LVEF}(\mathrm{l})$ (O or 1 )

Rhythm disonders $(0)$ Complications (O) and LVEF (O)

Rhythm disonders (I) Complications (0) 15 and LVEF ( I ) Rinythm disonders (2) Rhythm disorders (2) and LVEF (I)

Rhythm disorders (O) Complications (2) 12 and LVEF (O)

Rhylhm disorders (l) Complications 38 or LVEF (I) (Oor $)$

Rhyllim disorders (1) Complications 9 or $\operatorname{LVEF}(I)$ (Oor I)

Rhythm aisorders (I) Complications (0) 15 and $L V E F(I)$ Rhythm disorders (2) Rhythun disorders (2) and LVEF (I)

Rhythin disorders (I) Complications(I) 10 and LVEF (I) Rhythm disorders (2) Rhythm disorders (2) and LVEF (I)

Rhythm disorders (1) Complications (2). andior LVEF (I) Rhythm disorders (2) 


\begin{tabular}{|c|c|c|c|c|}
\hline \multirow[b]{2}{*}{13} & \multicolumn{2}{|r|}{$\begin{array}{l}\text { Rhythm disorders (2) } \\
\text { and LVEF (I) }\end{array}$} & \multirow[b]{2}{*}{ Complications (1) } & \multirow[b]{2}{*}{9} \\
\hline & $\begin{array}{l}\text { Location ischemia (I) or } \\
\text { Ischemic pain (1) }\end{array}$ & $\begin{array}{l}\text { Rhythm disorders (I) } \\
\text { and LVEF (I) } \\
\text { Rhyrhm disorders (2) } \\
\text { Rhythm disorders (2) } \\
\text { and LVEF (I) }\end{array}$ & & \\
\hline 14 & $\begin{array}{l}\text { Location ischemia (I) and } \\
\text { Ischemic pain (I) }\end{array}$ & $\begin{array}{l}\text { Rhythm disorders }(l) \\
\text { and } \\
\text { Rhyth LVEF disorders (2) } \\
\text { Rhythm disonders (2) } \\
\text { and LVEF (I) }\end{array}$ & $\begin{array}{l}\text { Complications } \\
\text { (1 or } 2)\end{array}$ & 7 \\
\hline 15 & $\begin{array}{l}\text { Location ischemia (l) or } \\
\text { Ischemic pain (l) }\end{array}$ & $\begin{array}{l}\text { Rhythm disorders (1) } \\
\text { andVor LVEF (I) } \\
\text { Rhythm disonders (2) } \\
\text { Rhythm disorders (2) } \\
\text { and LVEF (I) }\end{array}$ & Complications (2) & 11 \\
\hline 16 & Deceased within ten days & & & 43 \\
\hline 17 & Deceased after ten days & & & 7 \\
\hline 18 & Unilassiffiable patients & & & 19 \\
\hline \multicolumn{5}{|c|}{ 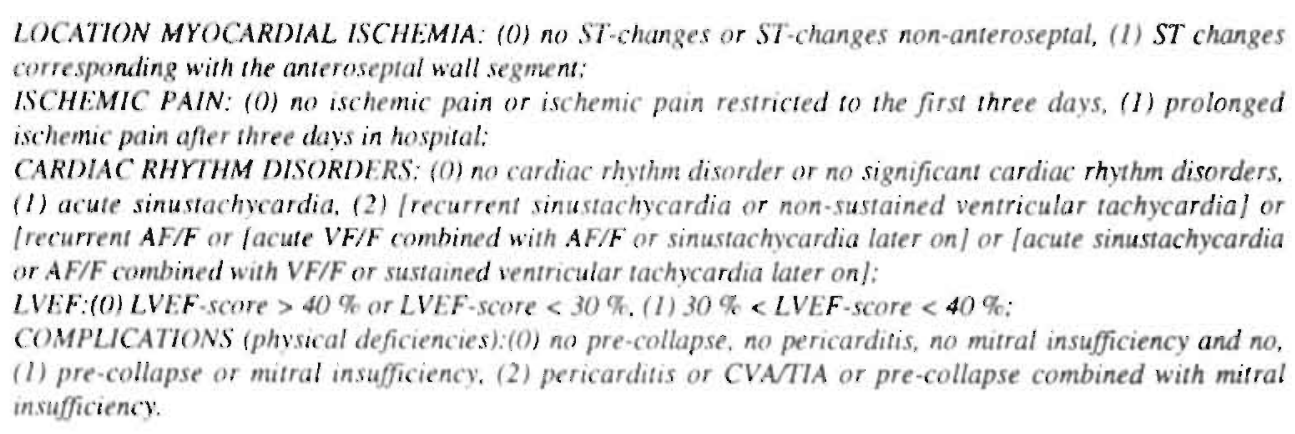 } \\
\hline
\end{tabular}

\subsection{Comparison of the predictive validity of the Severity of Illness-classification and that of Diagnosis Related Groups-classification.}

The mean standard deviation and number of patients for length of stay specified to the Severity of I!lness Classification and the DRG classification are presented in tables 8.3 and 8.4. The severity caregories turn our to be rather homogenous within each group regarding the length of stay, i.e. the values of the standard deviations in length of stay within each severity category appear to be rather narrow. Patients belonging to categories 1 and 2 
( $n=166$ ) will have the lowest length of stay of: about 8 days. Patients belonging to categories $3,4,6$ and $7(n=102)$ will have a mean length of stay of about 10 days. A smaller group of patients belonging to the severity categories 8 and $12(n=46)$ will have a mean length of stay of about 12 days and patients belonging to severity categories $5,9,11$ and $13(n=41)$ will stay about 13 days. Patients belonging to severity category $10(n=15)$ will have a very long stay of about 16 days. Patients belonging to severity category 14 and $15(\mathrm{n}=18)$ will have an extremely long stay of about 24 days. Patients who die soon after the infaretion (severity category 16) will have a very short length of stay of approximately 3 days in comparison to patients who die after a protracted illness (severity category 17), whose stay tends to amount to approximately 24 days.

Table 8.3 Mean, standard deviations and number of patients regarding length of stay by the Severity of Illness Classification (SIC).

\begin{tabular}{|c|c|c|c|}
\hline Severity Category & Mean & Sid Dev: & Freq. \\
\hline SIC-LOSI & 8.37 & 2.82 & 108 \\
\hline SIC-LOS2 & 8.76 & 3.46 & 58 \\
\hline$S I C \cdot \operatorname{LOS} 3$ & 9.92 & 2.71 & 12 \\
\hline SIC-LOS 4 & 10.10 & 3.83 & 63 \\
\hline SIC-LOS 5 & 12,85 & 2.83 & 13 \\
\hline SIC.LOSO & 10.33 & 3.33 & 15 \\
\hline SIC-LOS 7 & 10.58 & 4.08 & 12 \\
\hline SIC-LOS 8 & 12.21 & 3.55 & 38 \\
\hline SIC-LOS 9 & 13.67 & 4.79 & 9 \\
\hline$S I C \cdot \operatorname{LOS} 10$ & 16.33 & 4.55 & 15 \\
\hline$S I C \cdot \operatorname{LOS} 11$ & 12.70 & 3.95 & 10 \\
\hline$S I C-\operatorname{LOS} 12$ & 11.88 & 5.19 & 8 \\
\hline$S I C-\operatorname{LOS} 13$ & 13.56 & 4.78 & 9 \\
\hline$S I C-\operatorname{LOS} 14$ & 24.43 & 5.53 & 7 \\
\hline$S / C-\operatorname{LOS} 15$ & 24.00 & 9.61 & 11 \\
\hline SIC. LOS 16 & 3.44 & 2.54 & 4.3 \\
\hline SIC. $\operatorname{LOS} 17$ & 24.00 & 6.00 & 7 \\
\hline Subtotal & 10.37 & 5.12 & 438 \\
\hline unclassifiable & 16.00 & 8.04 & 19 \\
\hline Total & 10.61 & 5.95 & 457 \\
\hline
\end{tabular}

According to the DRG-classification of the same patient population (table 8.4) the mean length of stay of patients belonging to DRG 121, "complicated AMI' $(n=87)$ appears to be approximately 15 days and that of patients belonging to DRG 122 , 'non-complicated infarction' $(n=300)$ this amounts to approximately 10 days. Deceased patients belonging to DRG 123 have a mean length of stay of approximately 6 days. The standard deviations in length of stay within each DRG are rather high especially in DRG 121 and 123, this in contrast to the standard deviations in the Severity of Illness Classification. 
Table 8.4 Mean standard deviations and number of patients regarding length of stay by the Diagnosis Related Group classification (DRG).

$\begin{array}{llrrr}\text { DRG Category } & \text { Mean } & \text { Std. Dev. } & \text { Freq. } \\ 121 & \text { complicated AMI } & 14.78 & 7.26 & 87 \\ 122 \text { non-complicated AMI } & 9.78 & 3.74 & 300 \\ 123 \text { deceased } & 6.32 & 7.79 & 51 \\ & & & 438\end{array}$

Eta: $: 18.3 \%$ (unclassifiable patients $(n=19)$ and outliers $(n=7)$ excluded.

Variance of length of stay explained by the Severity of Illness Classification amounts to 58.3 $\%$, in comparison to $18.3 \%$ explained by the DRG classification. Next, a two-way analysis of variance is performed to evaluate the discriminatory power of both classifications. Results of this analysis are presented in table 8.5 .. Both the Severity of Illness Classification as well as the DRG classification appear to offer significant explanations for length of stay. This means that, given the severity of illness classification, the DRG classification offers an additional prediction in length of stay. The two-way interaction effect of SIC-LOS * DRG also appears to be significant. This means that the combination of both systems of classification will add a significant part to the prediction of length of stay. A specific analysis was done to pinpoint the way both systems complement each other. Úsing dummy regression analysis the main effect of DRG was differentiated into two 0-1 variables in which the non-complicated AMI-category is seen as the 'baseline'. The main effect of SICLOS was differentiated into sixteen 0-1 variables in which the "normal' category 1 is seen as the 'bascline'. After the differentiation all possible (14) interaction effects are computed. All 32. dummy variabies are brought into the regression analysis using length of stay as a dependent variable. It appears that only two interaction effects are significant at the $5 \%$ level and that these concem the parameters belonging to category 13 and 14 in the SIC-LOS classification coupled with the complicated AMI group in the DRG classification. So, it seems that differences in the definition of 'complications' between the SIC-LOS classification and the DRG classification will explain this effect. Patients are categorized in DRG 121 'complicated AMl' based on one (or more) specific complications; a list of 40 specific cardioyascular complications is used. some of which are not specifically investigated in this siudy (i.e. aneurysm heart wall, coronary vessel aneurysm, pulmonary embolism, cardiac arrest, papillary muscle rupture and açute renal failure).

The total variance explained by SIC-LOS and the DRG classification amounts to $63.2 \%$. The SIC-LOS classification, however, proves to explain much more of the variance than the DRG classification. Variance explained uniquely by the SIC-LOS classification is $42.2 \%$ and variance explained uniquely by the DRG classification amounts only to $2.2 \%$. Thus, it may be concluded that next to the SIC-LOS classification the DRG classification will give a very small but significant additional extra prediction in length of stay. 
Table 8.5 Results of a two-way analysis of variance using length of stay as dependent variable and the classifications of SIC-LOS and DRG as predictors. Unclassifiable patients in SiC-LOS $(n=19)$ and outliers $(n=7)$ have been removed from analysis. Adjusted sums of squares method, $n=438$.

$\begin{array}{lrrrrr}\text { Source of variation } & S S & d f & M S & F & P \\ \text { Main effects: } & 8705.3 & 18 & 483.6 & 37.0 & <.001 \\ \text { SIC-LOS } & 6069.7 & 16 & 379.4 & 29.1 & <.001 \\ \text { DRG } & 320.2 & 2 & 160.1 & 12.3 & <.001 \\ \text { Interaction effect } & & & & 2.1 & <.01 \\ \text { SIC-LOS * DRG } & 391.7 & 14 & 28.0 & 21.8 & <.001 \\ \text { Explained } & 9097.0 & 32 & 284.3 & & \\ \text { Residual } & 5289.4 & 405 & 13.1 & & \\ \text { Total } & 14386.3 & 437 & 32.9 & & \end{array}$

We do believe that other types of classifications of AM! patients must be constructed if one wishes to analyze specific costs of medical care in a valid way. Nevertheless, we have compared the value of the SIC-LOS classification and the DRG classification in predicting both the overall costs of medical care and specific costs of medical care. Results from this comparison are summarized in table 8.6 .

Table 8.6. Comparison of the predictive value in specific costs and overall costs of medical care in AMI patients classified according to SICLOS and DRG (outliers and unclassifiable patients excluded), $437 \leqslant n \leqslant 445$.

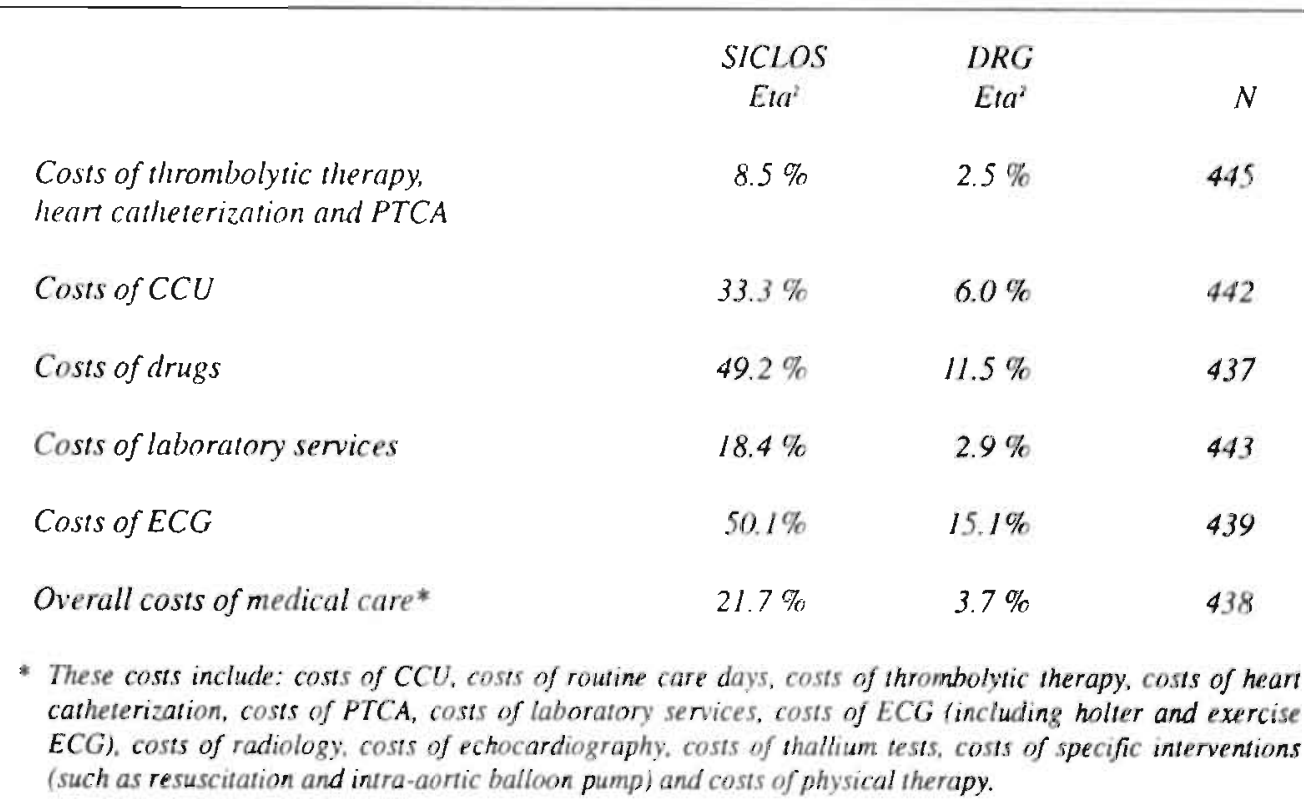


To obtain a comparison of the predictive validity of other patient classification systems (with regard to hospital costs), results from the study' performed by Thomas e.a. (1986,1991) may be used. In this study different classification systems were applied to the same data set derived from several hospitals. The: validity of these systems to predict costs of patients classified in the three. DRG's 'Acute Myocardial Infarction' has been evaluated. Some results from this study are summarized in table 8.7. According to Thomas e.a. the capability of these classification systems to explain costs for AMI patients is relatively low, except for Coded Disease Staging. However, some caution is necessary when these results are compared to those found in this study. There appear to be important differences in the length of stay of AMI patients between American and Dutch hospitals; in the U.S.A. the length of stay is considerably lower. The SIC.LOS classification sofar is only capable to explain the length of stay in one Dutch hospital. Furthermore, the poor performance of these systems compared to SIC-LOS with regard to explaining overall costs of medical care may not be caused by the inadequacies of the systems themselves but may lie in the incorrect assumption that classifying patients according to the likelihood of death or the length of stay will automatically explain accumulated costs of medical care during a hospital stay. From. literature we have not been able to find studies which compare the predictive power with regard to the length of stay for these systems for the same data-set of AMI-patients.

Table 8.7 Variance explained of total costs of medical care within adjacent DRG's 121-123 by different classification systems (adjusted for hospital differences).

Mosagkarion ugsiem

$\begin{array}{lcc} & R^{2} & N \\ \text { DRG } & 10.7 \% & 207 \\ \text { APACHE II } & 10.7 \% & 200 \\ \text { MEDISGRPS } & 16.4 \% & 173 \\ \text { Clinical Disease Staging } & 8.2 \% & 142 \\ \text { Coded Disease Staging } & 47.7 \% & 207 \\ \text { PMC } & 17.6 \% & 207\end{array}$

* Defued by Health Care Financing Administration (HCFA) used in cost-outlier points. Source: Thomas e.a.. 1986

\subsection{Applications of SIC-LOS.}

It is important that the application of a patient classification system is in accordance with the original purpose for which it is developed. The purpose of this study is to develop a method for Severity of lliness with the aim to explair differences in length of stay and (specific) costs of medical care. The possibilities in applicating such a system have not been investigated yet and furthermore the system has to be validated by using other data sets. Some thoughts about the application of the system will here be presented, but it is believed that the real value of it can only be established after several feasibility studies have been performed.

Our conclusion is that at least three different types of classification of AMI patients are: necessary if one aims to analyze cost differences in medical care during hospital stay in a 
reliable way. Only one is proposed in this thesis; the other two will still have to be developed in the near future.

One global set of clinical indicators is found to be relevant for this threefold classification (see table 8.1). Normally these indicators are not directly available to every caregiver or researcher in a computerized way but these are often collected by physicians on separate registration forms (certainly in the hospital under observation). The time necessary to collect these data from the medical record and the cardiology registration form by trained physicians is estimated to be $15-20$ minutes. However, data acquisition would certainly be facilitated, if the cardiology registration form would be available as computerized data. A computer program will have to be developed to classify patients automatically in the severity categories.

A severity of illness classification for AMI patients developed to explain costs of medical care can not be used to explain the likelihood of death. However, a part of the methodology used to define and operationalize severity indicators may be useful for this purpose. This would result in a research design that lays more emphasis on the clinical risk factors before hospital stay, but this would lead to a completely different study.

A patient classification system which classifies patients retrospectively is considered to be appropriate when applied within long term management of care; it is considered to be inappropriate for daily medical practice and for the treatment of individual case types. A severity of illness classification could serve as a supplement to well defined patien! catcgories in which patients are categorized according to their diagnoses (for instance the DRG-system). In that case a validated SIC-system may be used for:

* the evaluation of the case-inix and medical care.

Continuous evaluation of medical performance is supposed to be inherent to high quality of medical care. Evaluation of individual case types is often discussed by a group of medical professionals. A severity of illness classification may be helpful to evaluate medical performance on a departmental level; questions and outcomes may be discussed such as "what number and type of AMI patients have been treated during which period of time?"; 'are there changes in mortality rates or severity rates compared to fomer periods of time?'; "what are the consequences of changes in the AMI medical protocol on the distribution in catcgories of severity of illness and mean costs of care of these patients?'.

\section{* management of care.}

Changes in number and type of patients will affect nursing departments and ancillary departments. For instance, changes in severity of illness, in the sense that a substantially higher number of severely ill patients are treated, will certainly have an effect on the nursing bed requirement. A SIC may provide information that is important from a management point of view. Results of the SIC may help to answer questions like 'are there trends in the number of severely ill patients who need highly intensificd care or who need to stay in hospital for a long period?'.

\section{* clinical budgeting.}

From our results it may be concluded that severity of illness of patients is an important factor causing costs differences in medical care. When a hospital uses a classification system such as DRG for clinical budgeting, deviations from the budget may be traced to changes in the severity of illness of patients treated. Determination and evaluation of clinical budgets may improve when specific information on the distribution of severity of illness is available. 


\section{* utilization review.}

When patients are categorized in their severity of illness groups it is possible to correct the analysis of the utilization of hospital resources for this factor and find the real impact of organisational variables on the resource use.

\section{- quality of care studies.}

A severity of illness classification is often used in specific research programs to assess the quality of care given to clinical patients. Iezzoni says (1991) that in such studies: 'a severity standardization is necessary to control for the confounding influence of patient severity in comparisons of outcome that might be related to severity. Quality assessment generally focuses on the severity manifestations prior to medical intervention, assuming that once this severity is considered, residual poor outcomes are more likely to result from substandard care. Severity adjustments thus theoretically minimizes the potential for misjudgments about quality due to poor outcomes outside the control of the health care provider'.

\section{* inter-institutional comparison.}

It is believed that the major application of a SIC system will lie in specific scientific fesearch. For instance, it may be used to analyze differences in the severity of illness of patients having a similar diagnosis between general and university hospitals. Another application may be to analyze differences in costs of medical care between similar types of hospitals for severity-adjusted patient groups

\subsection{Conclusions.}

Results from empirical analysis have been used to develop a Severity of Illness Classification (SIC) aimed at explaining length of stay for AMI patients. The patient's severity of illness is regarded as a multidimensional concept. Six criteria were found to be important for explaining the patient's length of stay. To group AMI patients in clinically valid categories which are homogeneous with regard to length of stay, empirical combinations of the six criteria have been used to construct a Severity of Illness Classification. For the patient population at hand this has resulted in 17 categories classifying $95 \%$ of all AMI paticnts.

At this stage of research we are only able to compare the predictive validity of the SIC-LOS classification compared to the DRG system. Analysis of variance has shown that the prediclive validity of the SIC-LOS classification in terms of length of stay and costs is much higher than that of the DRG classification for the same patients. The predictive validity of the SIC. LOS in terms of costs of CCU and accumulated costs of acute care (thrombolytic therapy, heart catheierization and PTCA) was relatively low but still higher than the DRG's predictive validity. The latter is not surprising since our analysis showed that certain specific severity indicators did provide better explanations for these types of costs. Some indicators turned out to he typical for an increase of specific costs but did not have any effect on other types of costs. For instance if patients have a high CPK enzyme release they will stay longer at the CCU but this variable has generally no effect on length of stay. The severity of illness on admission will have an effect on the acute care costs, while, the severity during hospital stay (the course of the illness) will influence the patient's length of stay and other costs made during that stay. This might also be the reason that the predictive value: of the overall costs of care of AMI patients was rather low using the new classification. Similar results were found in other studies using other severity systems to explain overall costs differences in AMI patients. In order to reach a high predictive validity in explaining costs of medical care, the severity of illness of AMl patients will have to be defined in more specific ways dependent on the type of costs one intends to explain. 


\section{Conclusions and discussion.}

\subsection{Conclusions.}

\section{* Research objective.}

In the previous chapters an attempt has been made to explain cost differences in medical care given to hospitalized patients with Acute Myocardial Infarction (AMi). The central hypothesis investigated in this research is that the severity of illness is the crucial factor for variations in costs of medical care in patients having a similar discharge diagnosis. Secondly, the objective of this study is to develop a patient classification system for hospitalized patients with Acute Myocardial Infarction which classifies these patients according to their severity of illness in economically homogeneous categories.

\section{* Methodology.}

Relations between cardiac and cardiovascular deficiencies in AMI patients have been hypothesized in a causal model. The severity of several of these deficiencies (coronary stenosis, myocardial ischemia/necrosis, rhythm and conduction disorders, heari failure and specific complications) has been measured by means of specific clinical indicators. Each indicator is operationalized by means of three severity dimensions (intensity, extent and location) using severity weights derived from a panel of three cardiologists. The severity of illness has been measured by time-specific indicators (measured during several time periods within the hospital stay) and by dynamic severity indicators (reflecting changes in severity during stay).

Effects of the severity indicators on specific costs have been investigated. The analysis was performed for acute care costs, costs of CCU, length of stay and other costs of care. To guide the analysis of costs in medical care a general causal model has been developed which includes the clinical severity indicators and which at the same time has taken into account some non-clinical patient characteristics (like age, type of admission and type of discharge).

\section{* Results.}

\section{Acute Care Cosis}

Acute care costs have been analyzed separately as costs of thrombolytic therapy, costs of (acute) heart catheterization and costs of (acute) PTCA. The severity of illness turns out to have an effect on costs of thrombolytic therapy; ischemic pain on admission, ischemia over more wall segments and anteroseptally located necrosis as indicated by admission ECG results will increase the probability that costs will be induced due to thrombolytic therapy. Other factors, such as contra-indications (high age, being in coma and the duration of ischemic pain before admission) have a restrictive impact on costs of thrombolytic therapy. It was found that the severity of illness has a relatively weak effect on the costs of acute heart catheterization and the costs of acute PTCA. Medical treatment prior to the catheterization, i.e. thrombolytic therapy, turns out to have a relatively high predictive value for these costs. The severity of illness on the moment of admission can be seen as an indirect cause for these costs; more severely ill patients tend to get thrombolytic therapy and as a result of this they will get an acute heart catheterization. Furthermore, for patients with one occluded coronary vessel the probability of getting an acute PTCA will increase. It was concluded that the severity of illness measured on the moment of admission will explain costs of acute care to some extent. 
Costs of $\mathrm{CCU}$ and length of stay.

Severity of illness has a relatively large effect on the length of stay and the costs made in the Coronary Care Unit (CCU). More specifically, factors like anteroseptally located ischemia measured by ECG, specific cardiac rhythm disorders, left ventricular heart failure and pericarditis will substantially' increase the length of stay and the CCU costs. A high enzyme release, specific cardiac conduction disorders and anteroseptally located cardiac wall motion abnormalities prove to be typical for an increase in the CCU costs. Furthermore, suffering from pre-collapse, having ischemic pain, having mitral insufficiency or CVA/TIA will lead to a longer stay in hospital. It is concluded that the severity of illness certainly has a large effect on the CCU costs and the length of stay. However, severity indicators explaining length of stay turned out to be different from severity indicators explaining CCU costs.

Other costs of medical care.

Other costs of medical care have been separately investigated as costs of laboratory services, drugs, ECG, echocardiography and exercise tests. Costs of laboratory services were partly explained by the severity of illness. For the patient population in this survey it turned out that enrollment in a clinical trial will increase the costs of laboratory services substantially. Costs of drugs and costs of ECG explained in a more indirect way by severity of illness. These costs turned out to be strongly related to the length of stay; severely ill patients tend to stay in hospital longer and as a result of this costs of drugs and costs of ECG's will be high. Furthermore, when patients stay in CCU longer, chances of getting an echocardiography will increase. Apart from this, several specific severity indicators produce an additional increase in these costs. Costs of exercise tests are hardly influenced by severity of illness; these tests are normally planned for every patient just before hospital discharge. Some severity indicators turned out to influence the costs of exercise tests in a negative way. These tests are normally not performed if the patient suffers from specific severity conditions (contraindications).

\section{Background characteristics.}

Patient's background characteristics turned out to have almost no predictive value for costs of medical care, if one controls for his or her severity of illness. The only exceptions were the patient's age and sex; older patients usually tend 10 get no exercise test and female paticnts appear (quite unexplicably) to have lower chances of getting an acute heart calleterization.

\section{Survival/Death.}

Next to the severity of illness, a conditional factor influencing costs of medical care considerably was the death of patients during stay. Patients who die early on during hospital stay will of course have very low costs in contrast to surviving patients. Surviving patients will have lower cost compared to patients who die in hospitall after protacted illness. The severity indicators defined in this study are not able to explain the occurrence of death during hospital stay.

\section{* Classifying AMI patients according to their severity of illness.}

Within cosis analysis in AMI patients a major distinction had to be made between their severity of illness on admission and their severity of illness during hospital stay. In addition, the various types of costs of medical care can only be explained by severity indicators that characteristically belong to these cost lypes. For AMI patients many types of classifications according to severity of illness can be constructed depending on the types of costs that need to be explained. 
For the time being a single severity of illness classification (SIC-LOS) for AMI patients is proposed based on empirically determined severity criteria, which turned out to have a significant effect on the patient's length of stay. Severity of illness in this context is seen as a multidimensional concept; the degree and the combination in which medical deficiencies are found in AMI patients is important to classify them into a number of severity categories. In the SIC-LOS classification, 17 severity categories have been distinguished. Data analysis has shown that the SIC-LOS classification offers a better explanation for length of stay, costs of drugs, costs of laboratory services and costs of ECG $\left(\mathrm{Eta}^{2}\right.$ is resp. $58.3 \%, 49.2 \%$, $18.4 \%$ and $50.1 \%$ ) than the DRG-classification (Eta ${ }^{2}$ is resp. 18.3\%, 10.4\%, $2.9 \%$ and $15.1 \%)$. The predictive validity of SIC-LOS in terms of explaining CCU costs and accumulated costs of acute care (thrombolytic therapy, acute heart catheterization and acute PTCA) was much lower, but still higher than that of the DRG-system. The decrease in explained variance by the SIC-LOS classification is not surprising since our data analysis showed that other severity indicators were giving better explanations for these types of costs.

It can be concluded that in order to reach a high predictive validity in explaining medical care costs, the severity of illness in AMI patients has to be defined in various ways. depending on the type of costs one wants to explain. This can also be seen as the reason why one overall severity of illness classification with a high predictive validity for the total hospital costs of medical care is a goal which is difficult or even impossible to reach.

This study has shown that the severity of illness of patients is certainly a factor to be considered in costs-analysis of their medical care. However, the patient's severity of illness is not an absolute, universal and unidimensional concept as is often assumed. The severity of illness is found to be a very complex, dynamic and multidimensional concept which can be defined in various ways. In cost-analysis a differentiation of specific types of care will be necessary to understand the complex interplay between severity characteristics and costs. However, we do believe that the approach used in the development of severity indicators, the analysis of costs of care and the classification of patients into severity categories may serve as a general methodology to explain specific types of cost in medical care in other patient groups as well.

\subsection{Discussion.}

In order evaluate the methodology and results in this study some general criteria for patient classification systems as discribed in $\$ 2.1$. and some other issues will now be discussed.

\section{* Medical Meaningfulness/Content Validity.}

The medical meaningfulness/content validity refers to the definition of the severity weights used for the indices and the eventual recoding and classification of AMI patients. The severity weights were established after individually consulting three cardiologists. The proposed weights must be seen as an attempt to create a relative severity scale and cannot be regarded as a 'golden standard'. Standardization in severity measures is and always will be a goal difficult to attain.

The SIC-LOS classification does have a high degree of clinical credibility because it is based on clinical indicators which are normally used by cardiologists to diagnose and treat the patient. It is believed that the severity categories are still recognizable for cardiologists because they are based on empirically observed patterns of severity-scores within the population under survey. However, further research using other data sets combincd with ongoing critical evaluation from physicians is necessary to ascertain the medical content validity of our severity scales and the severity classification. 


\section{* Economic Meaningfulness/Predictive Validity.}

The predictive validity in this study refers to the degree to which the severity indicators used in this study are able to explain costs differences. The variance explained is found to vary depending on the type of costs. The explanation of costs of acute care by means of the severity indicators has the lowest predictive validity. Variance explained by the severity indicators regarding costs of CCU and length of stay (which will by assumption indirectly explain other costs of care such as drugs, ECG etc.) is relatively high.

\section{* Reliability.}

This study was limited to patients belonging to the DRG's 'acute myocardial infarction' admitted to one university hospital in the Netherlands during a specific time period of 16 months. The results may be influenced by local hospital practice. The results could have been different in other hospitals and/or during other periods of investigation. The reliability of results can only be evaluated, if comparable studies show similar results. Another problem is that the proposed Severity of Illness Classification for AMI patients has been based on results from data analysis of the same data set. In order to test the reliability of our Severity of Illness Classification, the same classification rules should be applied to a different set of data. Therefore, this study must be seen as a first investigation to obtain insight into the factors that influence costs and to classify patients according to their severity of illness.

The reliability of our results may also be influenced by the quality of the data used. The data quality depends for a great part on the scoring technique of data. In this study, physicians who were especially instructed for this purpose have performed the scoring of our data retrospectively (after the patient's discharge) using the patients' medical records as main source. Thus, the data collection was based on sources normally meant for clinical use. Furthermore, some severity indicators found to be important for cost explanation may have been influenced by retrospective interpretation of medical data. For instance the scoring of ECG-data was found be inconsistent in some cases. A more appropriate design would include a prospective way of data collection with especially instructed physicians, but this may be very difficult to achieve for acute illnesses like myocardial infarction. Another bias in the data may have resulted from the interpretation of test results by the cardiologists. At this stage of the research it was not possible to judge the impact of errors in the scoring of data on the results of the data analysis and the eventual classification. Lastly, the severity indices used in this study have been based on results of diagnostic tests commonly available at the time of investigation. New diagnostic technologies in the field of cardiology may give additional and more reliable data for severity criteria. New treatment technologies may also affect the relation between severity of illness and the costs of medical care. In further studies is it necessary to take such specific changes in diagnostic and treatment practice into account.

\section{* Administrative Relevance and Acceptability.}

From a strict point of view a patient classification can only be used for the purpose for which it was originally developed. The proposed SiC-LOS may therefore only be used to evaluate and compare the length of stay (and costs strictly related to length of stay such as costs of ECG, drugs and laboratory services) in AMI patients classified according to the DRG-system. To evaluate and compare acute care costs and CCU costs another patient classification must be used. which may or may nog have a relation with the first classification. So, for general policy and administrative purposes within hospitals the added value of a severity of illness system has to be evaluated in terms of the extra effort or costs necessary to construct and apply such a system. Although it is believed that a severity of illness classification may be useful for internal hospital management, we expect that its value is much higher in specific scientific studies, such as analyzing differences in severity and costs between various hospital institutions for a well defined patient group. 


\section{* Versatility and Flexibility:}

The requirement of versatility and flexibility refers to the capability of a classification system of combine categories in various ways of form new classifications for other goals. The SIC-LOS as presented in this study may only serve as a tool to analyze the length of stay in AMI patients. Combination or aggregation of the 17 categories to test the feasibility for other purposes has not been employed. However, it is found that for various costsanalysis several classifications of AMI patients must be determined. In our opinion versatility and flexibility must refer to the set of clinical indicators necessary for a multipurpose classification of patients. For cost analysis of medical care of AMI patients the clinical indicators as described in table 8.1 may be suitable. Further studies may reveal severity indicators that are important in explaining death/survival or the outcome in terms of the improvement in the patient's condition.

\section{* Costs-effectiveness and Practicality.}

Specific types of medical data are necessary to determine the categories in the proposed severity of illness classification. Normally these data are not available in a computerized form. The time necessary to collect these data from the medical record and the cardiology registration form by trained physicians is estimated to be similar to other severity systems based on clinical data (approximately 15-20 minutes). However, data collection would certainly be facilitated, if a computerized cardiology registration form would be available. Furthermore, a computer program has to be developed to classify patients automatically into severity categories. At this stage of the research this has not been performed. It is believed that the validity of the severity classification(s) should be assessed first by means of a multi= centre survey.

\section{Differences between the SIC-system and other disease-specific severity systems.}

In contrast to ordinal and unidimensional severity classifications like CSI, Apache II and Medisgrps, the level of measurement of the Severity of Illness Classification (SIC) proposed in this study is half nominal/half ordinal and it has a multidimensional character. We think that the SIC classification will provide certain advantages. First of all, the classification will give a clinical typology of patients according to their severity characteristics. Other severity classifications are not intended as case type classifications. They classify patients into severity categories ranging from level 1 to level 4 without referring to a clinical typology of patients within each category. Secondly, the SIC-classification is based on empirically observed combination of scores on severity indicators. Other severity systems have at tendency to use linear weights to accomplish a unidimensional scalc in the measurement of severity of illness. In almost all cases a justification of these weights is not given. There are. also differences in the methodology used to find relevant criteria for costs differences. The SIC-LOS classification is based on criteria derived from a interactive combination of results obtained from empirical cost analysis and professional judgement. Other systems are generally only based on consensus judgment of panels of clinicians and are originally not developed for economic purposes. At this stage of the research it is not possible to compare the systems using criteria such as content validity of measurement and preditive validity. 


\section{Costs of medical care.}

Costs of medical care for AMI patients have been specified into specific types of costs, and data analysis has been performed for each type separately. No data analysis has been done involving the total costs of medical care seen as an aggregated dependent variable. In our opinion overall cost analysis tends to conceal explanations for specific medical care costs. Overall cost analysis will place greater weight on those subcosts that are relatively high. As was found in this study, a specific type of costs can only be explained by a characteristic set of predictors. One unique set of predictors explaining total costs of medical care could not be established. Also, sets of predictors could not be interchanged in the explanation of specific types of costs. Such an exchange will eventually harm the predictive validity of the classification itself, because it will uses criteria not confirmed in empirical data analysis.

Costs of medical care have been determined for each patient by multiplying the number of services registered by the billing system with a service-related-cost-price. Normally these data are collected for reimbursement purposes. The investigated costs of medical care are therefore limited to chargeable services as specified by the billing system. Costs include only product-related material and personnel costs based on studies performed within the hospital under survey. These cost components can only be used for short term applications. For long term applications, capacity and overhead costs also need to be incorporated. Strictly speaking, no generalizations can be made with regard to other hospitals in which costs may be different for the same service.

\section{Unexplained variance.}

The indices of severity of illness can, of course, not explain the total variance of the dependent variables. The unexplained variance may have been caused by specific department characteristics or by variations in the (quality of) medical care given to patients by individual physicians. Unexplained variance will presumably also be caused by specific illness conditions not captured by our severity indicators and other characteristics of individual patients.

\section{Recommendations.}

It is clear that differences in costs of medical care between clinical patients can only be partially explained by the DRG-classification. Other factors, such as (changes in) severity of illness do contribute importantly to the explanation of costs in medical care. The DRGsystem is found to be an adequate tool to describe and analyse a total hospital population. However in some DRG's a further specification according to a severity of illness is necessary in order to reach a high predictive validity. A severity of illness classification may thus only be relevant for those patient categories which show large variations in length of stay and costs of medical care. Generally speaking one can state that the DRG-classification proves to be satisfactory in explaining costs of surgical patients, this in contrast to patient categories of non-surgical patients. We think that some adjacent DRG's with non-surgical patients must be complemented with a severity of illness classification based on clinical indicators, for this will certainly increase both the medical meaningfullness as well as the predictive validity. For instance, the DRG's involving non-surgical oncology patients or patients having chronic illnesses like renal failure (DRG 316 and DRG 317), medical back problems (DRG 234) and chronic obstructive pulmonary diseases (DRG 88) are examples of patient categories which, in general, show large variations in costs of medical care. Taking into consideration the complexity and the time-consuming activities necessary to measure and apply a severity of illness classification, it is recommended that further research must be concentrated on such specific heterogeneous patient categories within the DRG-system. 
The DRG-system is normally used as an instrument for prospective reimbursement of hospitals; specific reimbursement rates have been established for each DRG separately. The reimbursement rates do rely for a large part on the ability of the DRG-system to predict the length of stay. Costs of medical care are supposed to be related to the length of stay. However, for AMI patients the predictive validity in explaining overall cosis as well as specific costs of care by the DRG-classification is rather low If the goal of prospective reimbursement is to finance hospitals in a reliable and fair way, the application of a patient classification system such as the DRG-system for this goal must be critically evaluated and handled. In our opinion further research is certainly necessary in order to achieve a valid patient classification system for this specific purpose. We believe that the DRG-system will show its value for purposes of general hospital administration. The DRG-information will enable hospital managers to get global information on the hospital patient population, their resource use, costs and needs in medical care. But additional information about the patient's severity of illness will be necessary to understand and control clinical and financial processes in a more valid way.

Furthermore, we believe that in the context of costs analysis of clinical patients, costs of care need to be specified into various types of costs in order to evaluate and compare patient populations within and between hospital settings. Only then a thorough understanding of the relation between illness characteristics and costs of medical care will be made possible.

Further research should concentrate on building. expanding and empirically testing diseasespecific models which define the complex interplay between a patient's severity of illness and the medical care provided. Doing research from this perspective will give valuable insight into the complicated medical care processes within hospitals. 



\section{Summary.}

An important premise underlying this study is the assumption that patient classification systems are used for managing medical care in hospitals (chapter 1).

There are two important prerequisites for setting up such patient classification systems. Firstly, patients classified in the same category must from a recognizable group from the medical perspective of the health care providers. At the same time patients must be classified into the same category from an economic point of view if they make comparable use of hospital resources. A number of patient classification systems developed in the United States are described and compared with respect to, amongst others, the criteria of medical and economic meaningfulness. One of the most well known systems is the Diagnosis Related Groups (DRG) system which classifies patients on the basis of discharge data such as diagnosis and surgical procedures plus the patient's age and discharge status. A study of the literature has revealed that a number of DRG's leave much to be desired in terms of economic homogeneity and moreover, that the clinical validity of these DRG's can be improved (chapter 2).

This thesis intends to determine the factors that provide an explanation of differences in costs of medical care for patients categorized according to the DRG system. The research population has been restricted to patients who are classified under the DRG's 'Aculc Myocardial Infarction' (AMI). The research population comprises a total of 464 nonsurgical AMI patients who were admitted to and discharged from the department of Cardiology at the University Hospital Maastricht between January 1, 1987 and April 30 , 1988.

It is hypothesized that the patient's severity of illness is the most important factor influencing differences in costs. In addition, individual background characteristics, contra-indications for treatment, participation in a research protocol (clinical trials) and admission and discharge criteria are also believed to be important in predicling differences in costs. Because of the dynamic interaction that can be expected to exist between the patient's medical condition and his/her treatment, the hospital stay has been divided into several periods (chapter 3 ).

For the research population, data were retrospectively collected from discharge abstracts, ancillary service records and medical records (chapter 4).

The assessment of the costs of medical care given to a patient can be realized by linking a cost-price per service to the empirically determined number of services. The procedure by which the costs of ancillary departments (such as the clinical chemistry laboratory, the heart function laboratory etc. are specified per service (such as blood tests. ECG's etc.) is described in chapter 5 . This chapter also deals with the way in which the severity of several cardiac and cardiovascular deficiencies relevant to AMI patients is operationalized. In severity of illness three interrelated dimensions are distinguished: the intensity, the extent, and the location-related vulnerability of a deficiency.

In chapter 6 both, the empirically determined costs of medical care and the severity of illness of the research population are described. The costs of medical care are specified as diagnostic costs (heart catheterization, laboratory tests, ECG's, echocardiegraphy and exercise tests), treatment costs (thrombolytic therapy, PTCA and medication) and nursing costs (CCU and length of stay). The severity of various cardiac and cardiovascular deficiencies is measured by linking results from diagnostic tests (laboratory results, catheterization results, ECG data, echocardiography and exercise tests), physical 
examination and medical history to severity weights for each deficiency a patient may have during a specific period of hospitalization. The way these periodic severity scores are combined, resulting in the measurement of a patient's medical deficiency over the entire hospital stay, is also explained in chapter 6.

in the data analysis (chapter 7) a distinction is made between costs closely related to the acute phase of hospitalization and costs related to the entire hospital stay.

Acute phase.

During the acute phase of hospitalization, the decision to administer thrombolytic therapy is made. Analysis has shown that the location of myocardial necrosis (anteroseptal or nonanteroseptal), the extent of the myocardial ischemia and the presence of ischemic pain on admission will influence the decision to administer thrombolytic therapy. Furthermore, specific contra-indications (ischemic pain for more than six hours, an age over 75 and being in coma) are also found to be important factors.

The costs of $a(n)$ (acute) heart cathetcrization are only partly explained by the severity of illness on admission. In addition to the extent of myocardial ischemia, the administration of thrombolytic therapy appears to be the most important factor influencing these costs: severely ill patients are more likely to need thrombolytic therapy and as a consequence of this a catheterization is more likely to be performed.

During heart catheterization a PTCA (Dotter procedure) can be performed. For patients with coronary stenosis in one coronary artery, the administration of thrombolytic therapy decreases the likelihood of incurring costs for a PTCA. Conversely, the intensity of stenosis and the location of myocardial necrosis increase the likelihood of getting a PTCA. These effects, however, are not found in patients with stenosis in two or three coronary arteries.

Entire hospital stay.

From the heginning of hospitalization, all AMI patients receive intensive nursing care in the coronary care unit (CCU): following a CCU stay which may vary in length, patients are placed in a regular nursing ward. Differences in CCU stay (and thus costs) are largely explained by the severity of several deficiencies. Factors such as anteroseptal ischemia in the myocardium, extremely high enzyme release (CPK-release), abnormal wall motion in the anteroseptal myocardium, specific rhythm and conduction disorders, heart failure and pericarditis each significantly increase CCU costs.

Differences in the total length of stay of AMI patients are explained by some of the same severity indicators mentioned above: anteroseptal ischemia in the myocardium, specific rhythm disorders, heart failure and pericarditis are found to influence length of stay. In addition to this, length of stay is substantially increased as a result of continuous ischemic pain after three days, having a pre-collapse during hospitalization and specific complications such as mitral insufficiency and CVA/TIA.

Other costs of medical care are largely related to length of stay. The severity of specific cardiac and cardiovascular deficiencies hardly increases the costs for laboratory services, ECG's and medication in a direct way.

The costs of exercise tests appear not to be influenced by severity of illness. These tests are normally applied to all patients, with the exception of a few patients who have contraindications.

Next to severity of illness, background characteristics of patients (such as age and gender) appear to have very litlle additional value for predicting costs. Older patients, for example, score relatively high on several severity indicators, which accounts for their higher costs.

The death of a patient during the hospital stay, however, does affect costs. In case of death, a distinction must be made between patients who die acutely and patients who die after a prolonged stay. 
The results of this study have been used to develop a severity of illness classification system for patients with AMI. On the one hand, this system will guarantee medical validity and on the other hand will group patients with a similar length of stay. On the basis of empirically found severity indicators which influence the length of stay, 17 categories of patients have been distinguished. This severity of illness classification (SIC) proves to offer a considerably better explanation for differences in length of hospital stay in comparison to the DRG classification of the same patients, even when corrections are made for the number of categories that are used (chapter 8 ).

Finally, the most important conclusions of this study are presented in more detail in chapter 9. These conclusions are followed by a discussion of the results in the light of a number of methodological issues that are universally applicable to patient classification systems.

Insight into the complex relations, between severity of illness and costs of medical care is an essential prerequisite for the management of medical care within hospitals. Therefore improved patient classification systems can be a vital management instrument. 



\section{Samenvatting.}

Uitgangspunt van dit onderzoek is het gebruik van patiënt-classificatiesystemen ter besturing en beheersing van medische zorgverlening in ziekenhuizen (hoofdstuk 1).

Een belangrijk vereiste bij de opzet van zulke patiënt-classificatiesystemen is dat patiënten vanuit medisch oogpunt herkenbaar blijven voor zorgverleners. Daarnaası dienen patiënten uit eenzelfde categorie vanuit een economisch perspectief een vergelijkbaar middelenbeslag te hebben. Een aantal bestaande patiěnt-classificatiesystemen, alle ontwikkeld in de Verenigde Staten, wordt besproken en vergeleken aan de hand van deze beide criteria. Een van de meest bekende systemen is het zogenaamde Diagnosis Related Groups (DRG)systeem dat klinische patiénten classificeert op basis van. ontslaggegevens zoals de diagnoses, aard van operaties, leeftijd en wijze van ontslag. Uit een literatuurstudie blijkı dat niet alleen de economische homogeniteit van een aantal DRG's te wensen overlaat, maar dai eveneens de klinische herkenbaarheid of validitcit van deze DRG's verbeterd kan worden (hoofdstuk 2).

In dit proefschrift wordt nagegaan welke factoren een verklaring geven voor de verschillen in de kosten van de klinische zorgverlening tussen de patiènten, ingedeeld volgens de DRGsystematick. De onderzockspopulatie is beperkı tot patiënten die zijn ingedeeid in de DRG's 'Acuut Myocard Infarct' (AMI). Het betreft in total 464 niet-operatieve AMl-patiěnten, dic opgenomen en ontslagen zijn bij de afdeling Cardiologic van het Academisch Ziekenhuis Maastricht tussen 1 januari 1987 en 30 april 1988.

De belangrijkste factor voor kostenverschillen tussen patiënten is volgens menigeen het verschil in de ernst van hun aandoening. Daarnaast wordt verondersteld dat individuele achtergrondkenmerken van de patiënt, contra-indicaties voor behandeling, declname aan bepaalde wetenschappelijk onderzoeken (medische 'trials') en opname- en ontslagkenmerken van belang kunnen zijn voor verschillen in kosten. Vanwege de te verwachten dynamische interactie tussen de klinische toestand van de patiënt en zijn/haar bchandeling is het ziekenhuisverblijf van deze patiënten opgedeeld in diverse perioden (hoofdstuk 3).

Voor de onderzoekspopulatie zijn retrospectief ontslaggegevens, verrichtingengegevens en gegevens uit het medisch dossier verzameld (hoofdstuk 4).

Door een kostprijs per verrichting te combineren met het empirisch gevonden aantal verrichtingen is het mogelik de totale kosten vour de medische zorg aan een patient in becld te brengen. De procedure volgens welke de kosten van de ondersteunende afdelingen (zoals laboratoria, hartunctie afdeling, etc.) zijn verbijzonderd natar de diverse verrichtingen (zoals bloedonderzoek, ECG, etc.) wordt in hoofdstuk 5 uiteengezet. In ditzelfde hoofdstuk wordt ook ingegaan op welke wijze de emst van de diverse hart- en vaataandoeningen, die relevant zijn voor AMI patiënten, wordt geoperationaliseerd. De ernst van elke aandoening wordt anderscheiden naar drie dimensies: de intensiteit, de uitgebreidheid en de locatie-gebonden kwetsbaarheid van de aandoening.

In hoofdstuk 6, passeren de empirische kosten voor de medische zorgverlening en de ernst van de aandoening van de onderzoekspopulatie de revue. De kosten van de medische zorgverlening zijn gespecificeerd naar kosten voor djagnostick (hartcatheterisatie, laboratoriumtesten. ECG, echocardiografie en inspanningsonderzoek), kosten voor behandeling (thrombolytica, PTCA en medicatie) en kosten voor verpleging (CCU- en verpleegduur). Daarnaast wordt de ernst van de diverse hart- en vaataandoeningen gemeten door resultaten van de diagnostiek (laboratoriumtesten, catheterisatieverslagen, ECG-data, 
echocardiografie en inspanningsonderzoek), lichamelijk onderzoek en anamnese te relateren aan ernst-gewichten. De ernst-scores zijn gemeten gedurende diverse opname-perioden en vervolgens samengevoegd tot de ernst van de aandoening over de gehele opname.

In de data-analyse (hoofdstuk 7) is een onderscheid gemaakt tussen kosten die samenhangen met de acute període van de opname en kosten die betrekking hebben op het totale zickenhuisverblijf

Acule periode.

In de acute periode van een opname wordt besloten of een patiënt in aanmerking komt voor het krijgen van een thrombolyticum (stolsel-oplossend middel). Uit de data-analyse blijkt dat de locatie van necrose in het myocard (anteroseptaal of niet), de mate van uitgebreidheid van ischemie in het myocard en de aanwezigheid van ischemische hartpijn bij opname een grote invloed hebben op het toedienen van thrombolytica. Daarnaast blijkt dat specifieke contra-indicaties eveneens van belang zijn (langer dan zes uur ischemische pijn, het ouder zijn dan 75 jaar en het feit of patiënten in coma zijn).

De kosten van een (acute) hartcatheterisatie worden deels verklaard door de uitgebreidheid van de ischemie in het myocard. Het toedienen van thrombolytica blijkt de belangrijkste factor te zijn voor deze kosienverschillen: emsíg zicke patiënten hebben een hogere kans op thrombolytica en als gevolg hiervan zal hij hen cerder een catheterisatie plaatsvinden.

Tijdens zo'n hartcatheterisatic kan een PTCA ('Dotter' procedure) worden toegepast. Bij patiènten met een kransslagadervernauwing in één coronaire arterie verkleint het toedienen van thrombolytica de kans op kosten voor een PTCA. De mate van stenose en de locatie van necrose in het myocard daarentegen verhogen de kans op kosten voor een PTCA. Bij patienten met een vernauwing in twee of úrie coronaire arterièn kunnen deze effecten echter niet worden aangetoond.

Totale ziekenhuisverblijf.

Vanaf het begin van het ziekenhuisverblijf vindt voor alle AMI patiënten cen intensieve verpleging plaats in de Coronary Care Unit (CCU); na konere of langere tijd worden de patienten daama verpleegd op de 'normale' verplecgafdeling. Verschillen in CCU-duur (en dus kosten) worden grotendeels verklaard door de ernst van diverse aandoeningen. Factoren zoals anteroseptale ischemie in het myocard, extreem hoge enzym-waarden ('CPK-release'), abnormale wandbeweging in het anteroseptale myocard, specifieke ritme- en geleidingsstoornissen, pompfunctiestoornissen en pericarditis blijken elk cen significant verhogend effect te hebben op de CCU-kosten.

De versehillen in totale verpleegduur bij AMI patiënten worden deels door dezelfde ernstindicatoren verklaard: anteroseptale ischemie in het myocard, specifieke ritme-stoomissen, pompfunctiestoornissen en pericarditis blijken eveneens van invloed te zijn op de opnameduur. Daarnaast blijkt dat aanhoudende ischemische hartpijn na drie opnamedagen, het hetben van een (pre)collaps gedurende de opname en specifieke complicaties zoals mitraalinsufficientic en CVATTIA de verpleegduur substantieel verlengen.

De overige kosten voor medische zorgverlening blijken grotendeels geassocieerd met de verpleegduur. De ernst van bepaalde hart- en vaataandoeningen heeft nauwelijks een direct verhogend effect op kosten voor laboratorium-onderzoek, ECG en medicatic.

De kusten voor inspanningsonderzock blijken eveneens nauswelijks beìnvloed te worden door de crnst van de aandoening. Met uitzondering van enkele patiënien (met contraindicaties) blijkt dit onderzock standaard bij elke patiënt plaats te vinden.

Naast de ernst van de aandoening, blijken achtergrondgegevens van de patiënt (zoals leeftijd en geslacht) nauwelijks of geen voorspellende waarde te hebben voor kosten. Oudere patiènten bijvoorbeeld scoren relatief gezien hoger op diverse ernst-indicatoren en vandaar dat ủe lecftiju van de patiënt geen extra verklaring voor verschillen in kosten voor zorgverlening verschaft.

Vain wezenlijk belang voor de kosten is echter wel het overlijden van een patiënt in het ziekenhuis waarbij een onderscheid gemaakt moet worden tussen patiënten die acuut bij opname overlijden en patiënten die overlijden na een ruim verblijf in het ziekenhuis. 
De onderzoeksresultaten zijn gebruikt om een ernst-classificatiesysteem voor AMI patiënten te ontwikkelen dat enerzijds de klinische herkenbaarheid zoveel mogelijk waarborgt en anderzijds zo goed mogelijk de patiënten classificeert die een gelijke verpleegduur hebben (hoofdstuk 8). Gebasecrd. op de empirisch gevonden ernst-indicatoren die van invloed zijn op de verpleegduur, worden 17 categorieěn van patiënten onderscheiden. Deze ernstindeling (SIC) blijkt een aanzienlijk betere verklaring te geven voor verschillen in verpleegduur vergeleken met de DRG-indeling voor dezelfde patiënten, ook als gecorrigeerd wordt voor het aantal categorieën dat gehanteerd wordt.

Tenslotte worden in hoofdstuk 9 de belangrijkste conclusies uit he! onderzoek gedetailleerd weergegeven. Tevens worden de resultaten van dit onderzoek ter discussie gesteld aan de hand van enkele methodologische criteria die algemeen gelden bij patiënt-classificatie systemen.

Inzicht in de complexe relaties tussen de ernst van de ziekte en kosten wordt verondersteld een belangrijke voorwaarde te zijn bij de besturing en beheersing van medische zorgverlening in ziekenhuizen. Intrinsiek verbeterde patiënt-classificatiesystemen kunnen hierbij een belangrijk hulpmiddel zijn. 


\section{Glossary.}

T()

T1

T2

T3

AMI

SIC

DRG

MDC

APACHE

MEDISGRPS

PMC

CSI

LMR

PTCA

ECG

ECHO

$\mathrm{CCU}$

ICU

CTHROM

CCATH

CPTCA

LOS

CCCU

CLAB

CECG

CRADIO

CHOLTER

CECHO

CBRUCE

CTHAL

CEXER

CDRUGS

COTHER

CPHYS

STENINT

STENEXT

STENLOC

ISEXT

ISLOC

BRINT

BREXT

BRLOC

BREXP
Time period 0 (six hours after admission)

Time period 1 (six hours-three days after adnnission)

Time period 2 (three-ten days after admission)

Time period 3 (ten days after admission-discharge)

Acute Myocardial Infarction

Severity of Illness Classification

Diagnosis. Related Group

Major Diagnostic Category

Acute Physiology and Chronic Health Evaluation

Medical Illness Grouping System

Patient Management Category

Computerized Severity of Illness

Landelijke Medische Registatie

Perculaneous Transluminal Coronary Angioplasty

Electrocardiogram

Echocardiography

Coronary Care Unit

Intensive Care Unit

Costs of Thrombolytic therapy

Costs of Heart Catheterization

Costs of PTCA

Length of Stay

Costs of CCU

Costs of Laboratory services

Costs of ECG's

Costs of Radiology

Costs of Holter ECG tests

Costs of Echocardiography

Costs of Bruce ECG tests

Costs of Thallium tests

Costs of Exercise tests

Cosi of Drugs

Costs of other interventions besides thrombolytic therapy, drugs and PTCA

Costs of Physical therapy

Coronary stenosis intensity (or degree)

Coronary stenosis extent

Coronary stenosis location

lschemia extent according to ECG at rest

Ischemia !ccation according to ECG at rest

Ischemia intensity according to Bruce-ECG

Ischemia extent according to Bruce-ECG

Ischemia location according to Bruce-ECG

Ischemia expansion; additional ischemia according to Bruce-ECG 


\begin{tabular}{|c|c|}
\hline ISTHEXT & Ischemia extent according to thallium test \\
\hline ISTHLOC & Ischemia location according to thallium test \\
\hline SGOT & Serum Glutamic Oxalacetic Transaminase \\
\hline CPK & Creatine Phospho-Kinase \\
\hline LDH & Serum Lactic Dehydrogenase \\
\hline SGPT & Serum Glutamic Pyruvatic Transaminase \\
\hline NETHEXT & Necrosis extent according to thallium test \\
\hline NETHLOC & Necrosis location according to thallium test \\
\hline ISPAIN & Ischemic pain \\
\hline CWMINT & Intensity (or degree) of cardiac wall motion abnormalities \\
\hline CWMEXT & Extent of cardiac wall motion abnormalities \\
\hline CWMLOC & Location of cardiac wall motion abnormalities \\
\hline LVEF & Left Ventricular Ejection Fraction \\
\hline LVEDD & Left Ventricular End Diastolic Dimension \\
\hline $\mathrm{VCl}$ & Collapse Vena Cave Inferior \\
\hline ISCRV & Ischemia right ventricle \\
\hline CWMRV & Cardiac wall motion abnormalities in right ventricle \\
\hline PALP & Palpitations \\
\hline DYSPN & Dyspnea \\
\hline PRECOL & (Pre)-collapse \\
\hline SYCOL & Syncope/collapse \\
\hline HBLPRES & High blood pressure \\
\hline LBLPRES & Low blood pressure \\
\hline AP & Angina pectoris \\
\hline LEDEMA & Lung edema \\
\hline BEDEMA & Body edema \\
\hline HEPTO & Hepatomegaly \\
\hline PERIC & Pericarditis \\
\hline MITINS & Mitral insufflciency \\
\hline CSHOCK & Cardiac shock \\
\hline CVA/TIA & Cerebral vascular accident/ transient ischemic attack \\
\hline FTHROM & Former thrombolytic therapy \\
\hline FCOR & Former coronary insufficiency \\
\hline FAMI & Former acute myocardial infarction \\
\hline FHYP & Former hypertension \\
\hline $\mathrm{AF} / \mathrm{F}$ & Atrial flutter/-fibrillations \\
\hline $\mathrm{VF} / \mathrm{F}$ & Ventricular flutter/-fibrillations \\
\hline RBBB & Right bundle branch block \\
\hline ADINT & Admission internal \\
\hline ADEXT & Admission external \\
\hline DEATH & Deceased during hospital stay \\
\hline HOME & Discharge to home \\
\hline TRANS & Transferred during hospital stay \\
\hline PRIMI & PRO-urokinase In Myocardial Infarction (clinical trial) \\
\hline
\end{tabular}





\section{Bibliography}

- Aert van JH, Ziekenhuis kosten in econometrisch perspectief. Thesis NZI, Utrecht, 1977.

- Alemi F, Rice J and Hankins R, Predicting in-hospital survival of myocardial infarction. A comparative study of various severity measures, Medical Care 1990:28:9:762-775.

- Aranow DB. Severity of illness measurement: applications in quality assurance and utilization review, Medical Care 1988:45:2:339-366.

- Averill RF, 'The design and development of the Diagnosis Related Groups (DRG'S), ' in Averill RF. Mullin RL and Elias ED, The revised ICD-9-cm Diagnosis Related Groups, Health systems International, New Haven, Connecticut, documentation to the May 1983 software.

- Ayanian JZ and Epstein AM, Differences in the use of procedures between women and men hospitalized for coronary heart disease. The New England Journal of Medicine 1991;325:4:221-225.

- Backofen J, Ashworth MA and Horn SD, The computerized severity index, Healthcare forum 1987:35-37.

- Barbash GI, Aafran C. Ransil BJ. Pollack. MA and Pasternack RC, Need for better severity indexes of Acute Myocardial Infarction under Diagnosis-Related Groups, American Journal of Cardiology 1987:59:1052-1056.

- Becker ER and Steinwald B. Determinants of hospital case-mix complexity. Health Services Research 1981:16:4:439-456.

- Berry RE, Product heterogeneity and hospital cost analysis, Inquiry 1970;7:67-75.

- Berry RE, On grouping hospitals for economic analysis. Inquiry 1973;10:5-12

- Bloomrosen MF and Kominski GF. Proceedings from propac's technical advisory conference on alternative case-mix classification systems. Prospective Paymenı Assessment Commission, Washington D.C.. 1987.

- Bohrnstedt G.W. and Carter T.M. Robustness in regression analysis, in: Costner H.L. (editor): Sociological Methodology 1971. San Francisco, 1971; 118-146.

- Braunwald E, A rextbook of Cardiovascular medicine, W.B. Saunders Company, Philadelpia, 1984.

- Brewster AC, Jacobs CM and Bradbury RC, Classifying severity of illness by using clinical findings, Health Care Financing Review 1984;107-108.

- Brewster AC, Karlin BG, Hyde LA, Jacobs CM, Bradbury RC and Chac YM, MEDISGRPS: A clinically based approach to classilying hospital paticnts at admission, Inquiry 1985;22:12:377-387.

- Brouwers HMP, Kostprijsonderzoek op de ondersteunende afdeling klinische farmacie. Master's thesis HEAO. Sillard, 1988.

- Burns LR and Wholey DR, The effects of patient, hospital and physician characteristics on length of stay and mortality, Medical Care 1991;29:3:251-271.

- Calore KA and Iezzoni L. Disease staging and PMC's; Can they improve DRG's, Medical Care 1987;25:8:725-735.

- Charbonneau C, Ostrowski C, Phoehner ET, Lindsay P, Panniers TL, Houghton P and Albright J. Validity and reliability issues in Alternative Patient Classification Systems, Medical Care 1988;26:8:800-810.

- Coclen RHM, Kostprijsonderzoek op het vaatlaboratorium, Master's thesis HEAO. Sittard, 1988.

- Coffey R, Non-surgical DRG's: should they be refined with disease staging? Paper presented at the Second Annual Meeting of the Association for Health Services Rescarch, Chicago, Illinois, 1985.

- Coffey $\mathrm{R}$ and Goldfarb MG, DRG's and disease staging for reimbursing medicarc patients, working Paper no 1. Hospital Studies Program, National Center for Health Services Research, Rockville, Maryland oct. 1984. In: Review of the literature on Diagnosis Related Groups by Worthman LG, Cretin S, RAND, 1986.

- Conklin JE, Lieberman JV, Barnes CA and Louis DZ, Disease staging: implications for hospital reimbursement and management, Healih Care Financing Review 1984:13-22.

- Conklin JE, DRG refinement: A feasibility assessment using stage of disease, age and unrelated comorbidity. Proceedings of the 1985 Public Health Conference on Records and Statistics DHHS Pub.no. 86-1214, 1985. 
- Connell FA, Blide LA and Hanken MA. Ambiguities in the selection of the principal diagnosis: impact on data quality; hospital statistics and DRG's, Journal of the American Medical Record Association 1984;55:2:18-23.

- Corn F, Quality control of hospital discharge data, Medical Care 1980;18:4:416-427.

- Corn F. The sensitivity of prospective hospital reimbursement to errors in patient data. Inquiry 1981;18:351-360.

- Cotterill P, Bobula J and Connerton R, A comparison of alternative DRG relative weights for the Medicare prospective payment system. Working paper, febr. 1985 In: Review on literature on Diagnosis Related Groups by Worthman LG, Cretin S, RAND, 1986.

- Davis K. Anderson G and Steinberg E. Diagnosis Related Group prospective payment: implications for health care and medical technology, Health Policy 1984;4:139-147.

- Demlo LK and Campbell PM. Improving hospital discharge data: lessons from the national hospital discharge survey "Medical Care 1981:19:1:1030-1040.

- Desharnais S, Kobrinski E, Chesney J, Long M, Ament R and Fleming S, The early effects of prospective payment system on inpatient utilization and the quality of care, Inquiry 1987:24:7-16.

- Desharnais SI, Chesney ID and Fleming ST, Should DRG-Assigment be based on age, Medicai Care 1988;26:2:124-131.

- Doremus HD and Michenzi EM, Data Quality; An illustration of its potential impact upon a Diagnosis-Related Groups case-mix index and reimbursement, Medical Care 1983;21:10:1001-1011

- Eagle KA, Mulley AG, Skates SJ, Reder VA, Nicholson BW, Sexton JD, Bamett GO and Thibault GE, Length of stay in the Intensive Care Unit, The Journal of the American Medical Association 1990;264:8:092-997.

- Factor $M$ and Kirk ES, Pathophysiology of myocardial ischennia, in: Hurst JW, The Heart, sixth edition, vol. 1. 1986.

- Fcinglass MA. Scheruhel JC and Swartz JA. Comparisan of fwo systems to meacure the severity of illness, Journal of the American Medical Record Association 1988;59:7:3438.

- Feldstcin MS and Schuttinga J. Hospital costs in Massachusetts: a methodological study, Inquiry 1977:14:22-31.

- Feller RB, Thompson ID, Averill RF, Freeman JL and Shin Y, Case-mix definition by diagnosis related groups, Medical Care supplement 1980;18:2:1-39.

- Fetter RB and Freeman JL. Diagnosis Related Groups: product-line management within hospitals, The Academy of Management Review 1986;11:1:41-54.

- Fox J, Regression diagnostics. Quantitative applications in the social sciences, Sage university paper, number 07-079, Beverly Hills, 1991.

- Frick AP, Martin GS and Shwaîtz M. Case-mix and cost differences between teaching and non teaching hospitals, Medical Care 1985:23:4:283-295.

- Fuster V. Frye RL, Connolly DC. Dancelson MA. Elveback LR and Kurland LT, Arteriographic pattems early in the onset of the coronary syndromes, British Heart Journal 1975;37:1250-1255.

- Garg ML, Louis DZ, Gliche WA, Spirka C.S. Skipper JK and Parekh RR, Evaluating inpatient costs: the staging mechanism, Medical Care 1978;16:3:191-201.

- Gemert G. Kwaliteitsonderzock LMR-gegevens: aantal fouten verschilt sterk per zickenhuis, Signet 1986;1:1-3.

- Gennert G, Kwaliteitsonderzoek LMR-gegeveris: pereentage vage hoofddiagnosen stjjgt, Signet 1986;3:13-14.

- Gensini GG. Coronary Arteriography. Futura Publishing Compagny, Inc., New York, 1975.

- Gertman PM and Lowenstein S, A research paradigm for severity of illness: issues for the Diagnosis Related Group system. Health Care Financing Review 1984:79-90.

- Goldiarb MG and Coffey RM, Case-mix differences between teaching and non teaching hospitals, Inquiry 1987:24:68-84.

- Gonclla IS and Zeleznik C. Prospective reimbursement using the DRG case-mix classification system. A medical perspective, paper presented at a symposium on contemporary issues in health care, 1983.

- Gonella IS. Hombrook MC and Louis DZ. Staging of disease. A case-mix measurement, The Journal of the American Medical Association 1984;251:5:637-644. 
- Gonella JS, editor, Clinical criteria for disease staging. Version 6.0. Systemetrics, Mcgraw-Hill, Santa Barbara, California, 1985.

- Goodisman LD. Hospital case mix and average charge per case: an initial study. Health Services Research 1979:44-55.

- Gorlin R, Fuster V and Ambrose A. Anatomic-physiologic links between acute coronary syndromes, Circulation 1986:74:1:6-9.

- Grimaldi PL and Micheletti JA. Diagnosis Related Groups. A practitioner's guide. Second edition, Pluribuss Press, Chicago, 1983.

- Groot LMJ, Patient-georiënteerde ziekenhuiseconomic; een poging tot synthese tussen professie en management. In: In het kader van de gezondheidszorg. Lochum. De Tijdstroom, 1978;183-198.

- Gustafson DH, Fryback DG, Rose JH. Yick V, Prokop CT, Detmer, DE and Moore J, A decision theoretic methodology for severity index development, Medical Decision Making 1986;6:1:27-35.

- Health Information Systems 3M-Health care, All patient grouper application; case-mix analysis using ICD-9-cm PC grouper software, Utah, 1991.

- Health Systems International, Diagnosis Related Groups, Definition manual, third revision, New Haven, Connecticut, 1986.

- Health System International, Computerized severity index; ICD-9-CM severity modification. New Haven, Connecticut, 1987.

- Health System Management Group, DRG refinement with diagnostic specific comorbidities and complications: a synthesis of current approaches to patient classification. School of Organization and Management, Yale University. New Haven. 1985.

- Health Systems Management Group, Refined Diagnosis Related Groups, version 2.3. definitions manual, School of Medicine, Yale University. New Haven, 1990.

- Horn SD and Schumacher DN, An analysis of case-mix complexity using information theory and diagnostic related grouping, Medical Care 1979;17:4:382-389.

- Horn SD and Schumacher DN, Comparing classification methods: measurement of variations in charges, length of stay, and mortality, Medical Care 1982;20:5 :489-500).

- Horn SD, Sharkey PD and Bertham DA, Measuring severity of illness: homogeneous case mix groups. Medical Care 1983;21:1:14-25.

- Horn SD and Sharkey PD, Measuring severity of illness to predict palient resource usc within DRG's, Inquiry 1983;20:314-321.

- Horn SD, Measuring severity of illness; comparisons across institutions, American Journal of Public Health 1983;73:1:25-31.

- Horn SD, Chachich B and Clopton C. Mcasuring severity of illness: a reliability study, Medical Care 1983;21:7:705-714.

- Horn SD, Horn RA and Sharkey PD. The severity of illness index as a severity adjustment to Diagnosis Related Groups. Health Care Financing Review Annual Supplement 1984;33-45.

- Horn SD, Bulkley G, Sharkey PD, Chambers AF, Horn RA, Schramm CJ, Interhospital differences in severity of illness; problems for prospective payment based on Diagnosis Related Groups, The New England Journal of Medicine 1985;313:1:20-24.

- Horn SD, Sharkey PD. Chambres AF and Horn RA, Severily of illness within DRG's, impact on prospective payment, American Journal of Public Health, 1985;75:10:11951199.

- Horn SD and Horn RA, The computerized severity index: a new lool for case mix management. Centre for Hospital Finance and Management, The John Hopkins University, Baltimore, Maryland, 1986.

- Horn SD. The computerized severity index: conceptualization, comparisons and applications. Centre for Hospital Finance and Management, The John Hupkins University, Baltimore, Maryland, 1986.

- Horn SD, Hom RA, Sharkey PD, Beall RJ, Hoff JS and Rosenstein BJ, Misclassification problems in diagnosis related groups, The New England Journal of Medicine 1986;20:8:484-487.

- Horn SD, Computerized severity of illness. Conference. In: Proceedings from propac's technical advisory conference on alternative case-mix classification systems by Bloomrosen MF and Kominski GF, 1987. 
- Horn SD, Sharkey PD, Buckle JM, Backofen JE, Averill RF and Horn RA, The relationship between severity of illness and hospital length of stay and mortality, Medical Care 1991;29:4:305-317.

- Hornbrook MC. Hospital case-mix: its definition, measurement and use: part I, the conceptual framework, Medical Care Review 1982;1-43.

- Hornbrook MC, Hospital case-mix: its definition, measurement and use: part II, review of alternative measures, Medical Care Review 1983;73-123.

- Hornbrook MC and Monheit AC, The contribution of case-mix severity to the hospital cost-output relation, Inquiry 1985;22:259-271.

- Hunt K, DRG what it is, how it works and why it will hurt, Medical Economics $1983 ; 262-269$.

- Hupperichs RHLM, Kostprijs op de ondersteunende afdelingen klinische farmacie, Master's thesis HEAO, Sittard, 1988.

- Hurst JW, The Heart, Arteries and Veins, McGraw-Hill Book Company, Inc., 6th ed. New York, 1986.

- Iczzoni LI, Ash AS, Cobb JL and Moskowiz MA, Admission medisgroups score and the cost of hospitalization, Medical Care 1988;26:11:1068-1080.

- Ic żoni LI, Using severity information for quality assessment: a review of three cases by five severity measures, Quality Review Bulletin 1989;15:12:376-382.

- Iezoni LI, Using administrative diagnostic data to assess the quality of hospital care, Pittfalls and potential of ICD-9.cm, International Journal of Technology Assessment in Health Care 1990;6:272-281.

- Iezzoni LI, Shwartz M, Moskowitz MA, Ash AS, Sawitz E and Burnside S, Illness severity and costs of admissions at teaching and non-teaching hospitals, The Journal of the American Medical Association 1990;264:11:1426-1431.

- Iezoni L.I, in: Health care quality management in the 21st century, edited by Congh JB. American College of Physicians Executives (ACPE), Tampa, Florida, 1991.

- Jeffers JR and Siebert CD. Measurement of hospital cost variation: case-mix, service intensity and input productivity factors, Health Services Research 1974:293-307.

- Jenks SF and Dobson A. Refining case-mix adjustment; the research evidence, The New England Journal of Medicine 1987:317:11:679-686.

- Jetien T. De kostensiructuur van de afdeling radiologie. Master's thesis Gezondheidswetenschappen, Rijksuniversiteit Limburg, 1987.

- Johnson AN and Appel GL. DRGs and hospital case records: implications for medicine case mix accuracy, Inquiry 1984;21:128-134.

- Jones KR, Predicting hospital charge and stay variation; the role of patient teaching slatus, controlling for Diagnosis Related Group, demographic characteristics and severity of illness, Medical Care 1985:23:3:220-235.

- Kamm V, Kostprijsonderzoek bloedtransfusiedienst, internal report Dienst Administratie en Bedrijfseconomie, academisch zickenhuis Maastricht, 1989.

- Kar v.d. JM, Kostprijsonderzoek haematologisch laboratorium, Master's thesis Bedrijfskunde TUE, Eindhoven, 1989.

- Keeler EB, Kahn KL, Draper D, Sherwood MJ, Rubenstein LV, Reinisch EJ, Kosecoff J and Brook RH. Changes in sickness at admission following the introduction of the prospective payment system. The Journal of the American Medical Association 1990;264:15:1962-1968.

- Kitchin AH and Pocock SJ, Prognosis of palients with acule myocardial infarction admilted to a coronary care unit, I: Survival in hospital, British Heart Journal 1977:39:1163-1166.

- Klastorin TD and Watts CA, On the measurement of hospital case-mix, Medical Care 1980;18:6:675-685.

- Kreitzer SL, Loebner ES and Roveti GC, Severity of illness: the DRG's missing link? Quality Review Bulletin 1982:8:5:21-34.

- Lave R and Lave B. Estimated cost functions for Pennsylvania hospitals, Inquiry 1970;7:2:3-14.

- Lave JR and Lave LB. The extent of role differentiation among hospitals, Health Services Research 1971:6:15-38.

- Lichting LK, Hospital information systems for case-mix management, John Wiley \& Sons, Inc., New York, 1986. 
- Limpens PGPJM, Kostenonderzoek op het klinisch chemisch laboratorium, academisch ziekenhuis Maastricht, Master's thesis Faculteit der Economische Wetenschappen. Katholieke Hogeschool Tilburg, 1986.

- Limpens PGPJM en Roberts SJGL, Uitvoering kostprijsonderzoeken volgens een globale methode, internal paper Dienst Administratie en Bedrijfseconomie, kenmerk: BE/PL/SR/PB 88.1078, academisch ziekenhuis Maastricht, 1988.

- Limpens PGPJM, Kostprijsonderzoek afdeling hartfunctie en longfunctie, internal report. Dienst Administratie en Bedrijfseconomie, academisch ziekenhuis Maastricht, 1988.

- Limpens PGPJM, Kostprijsonderzoek afdeling virologie en immunologie. internal report Dienst Administratie en Bedrijfseconomie, academisch ziekenhuis Maastricht, 1988.

- Lloyd S and Rissing JP. Physician and coding errors in patient records, The Journal of the American Medical Association 1985;254:10:1330-1336.

- Louis DZ. Disease staging: A clinically based approach to measurement of disease severity, Vol. 5: Reabstracting study, Sysiemetrics, 1984.

- Luke RD, Dimensions in hospital case-mix measurement, Inquiry 1979;16:38-49.

- Maes EJME, Kostprijscalculatie van de afdeling pathologie, Master's thesis HEAO. Sittard, 1987.

- Manton KG and Vertrees JC. The use of grade of membership analysis to evaluate and modify diagnosis related groups, Medical Care 1984;22:12:1067-1082.

- Mc.Guire TE. An evaluation of DRG-severity and complexity refinement, working paper, EEC concerted aclion. Barcelona, 1990.

- Mc.Mahon LF and Newbold R, Variation in resource use within Diagnosis Related Groups; the effect of severity of lliness and physician practice, Medical Care 1986;24:5:338-397.

- Mc.Neil BJ, Kominski GF, Williams and Ashman A, Modified DRG's as evidence for variability in patients severity, Medical Care 1988;26:1:53-61.

- MediQual Systems, Medisgroups; Managemeni Information System, Internal Paper, Chicago, 1985.

- MediQual Systems, Medisgroups; admission severity classification, Internal Paper, Chicago, 1985.

- Meyer J, (PRIMI Trial Study Group), Randomized double blind trial of recombinant prourokinase against streptokinase in Acute Myocardial Infarction, The Lancet 1989:1:863-867.

- Mitchell JB, Calore KA, Cromwell J, Freiman and Hewes HT, Creating DRG-based physician reimbursement schemes: a conceptual and empirical analysis "First year report to HCFA Grant number 18-p-98387/1-01, Center for Health Economics Rescarch, Chestnutt Hill, Massachusetts, 1984. In: Review on the literature on Diagnosis Related Groups by Worthman LG, Cretin S, RAND, 1986.

- Mitchell JB, Calore KA, Cromwell J, Freiman and Hewes HT, Physicians DRG's: Whal do they look like and how would they work draft, discussion paper, HCFA Grant number 18-p-98387/1-01, Center for Health Economics Research, Chesinull Hill, Massachusetts, 1985. In: Review on the literature on Diagnosis Related Groups by Worthman LG, Cretin S, RAND, 1986.

- Montfort van APWP, Production functions for general hospitals: an econometric analysis. Thesis, NZI, Utrecht, 1980.

- Moreau R, Soupison T, Vanqueling P. Derrida S, Beaucour H and Sicol C, Comparison of two simplified severity scores (Saps and Apache II) for patients with acute myocardial infarction, Critical Care Medicine 1989;17:5:409-413.

- Mullin RL, DRG's: A bricf description, Connecticul Medicine 1983;47:5:281-282.

- Mullin RL, Diagnosis Related Groups and severity; ICD-9-CM, the real problem, The Journal of the American Medical Association 1985;254:9:1208-1210.

- Nederstigt PFPM, Diagnosis Related Groups nader bezien, Gezondheid en Samenleving 1981:2:3:182-195.

- Nederstigt PFPM en Voss GBWE, Integratic van medische en economische informatie. komt binnen handbereik, Het Ziekenhuis 1985;17:2:61-65.

- Nederstigl PFPM. Diagnosis Related Groups; een patiëntgeoriënteerd kosteninformatiesysteem. Thesis, NZI, Tilburg, 1985.

- Nederstigt PFPM and Voss GBWE, Diagnosis Related Groups; klinisch en poliklinisch, Tijdschrift Sociale Gezondheidszorg 1985;63:12:476-483. 
- Omenn GS and Conrad DA, Implications of DRG's for clinicians, The New England Journal of Medicine 1984;311:12:1314-1317.

- Panniers TL. Severity of Illness, quality of care and physician practice as determinants of hospital resource consumption, Quality Review Bulletin 1987;13:5:158-165.

- Pass T, Case-mix severity systems provide DRG alternatives, Health Care Financial Management 1987;74-82.

- Patrick DL and Deyo RA, Generic and disease specific measures in assessing health status and quality of life. Medical Care 1988;27:3:217-231.

- Pettengill $j$ and Vertrees $J$, Reliability and validity in hospital case mix measurement, Health Care Financing Review, 1982;4:2:101-128.

- Plotnick GD, Fisher ML, Lerner B, Carliner NH, Peters RW and Becker LC, Collateral circulation in patients with unstable angina, Chest 1982;82:6:719-725.

- Pozen MW, D'agostino RB, Mitchell JB, Rosenfeld DM, Guglielmino JT, Schwartz ML, Teebagy N, Valentine JM and Hood WB. The usefulness of a predictive instrument to reduce inapproperiate admissions of the coronary care unit, Annals of internal medicine 1980;92:238-242.

- Prince AJ. Nieuwe gezondheidszorg in de V.S., kostenbeheersing en kwaliteitsbewaking via 'managed care', Medisch Contact 1987;8:239-242.

- PSRO ' $\mathrm{X}$ '. Evaluating Myocardial Infarction using the staging concept, Quality Review Bulletin 1981:7:11:15-20.

- Roberts SJGL. Kostprijsonderzoek bacteriologie en voedingsbodem/spoelkeuken, internal repori Dienst Administratic en Bedrijfseconomie, academisch ziekenhuis Maastricht, 1989.

- Roberts SJGL, Kostprijsonderzoek laboratorium voor weefseltypering, internal report Diensı Administratie en Bedrijfseconomic, academisch ziekenhuis Maastricht, 1989.

- Roberts SJGL en Kamm V. Kostprijsonderzoek operatiekamers, internal report, Dienst Administratie en Bedriffseconomic, academisch ziekenhuis Maastricht, 1990.

- Rogers WH, Draper D. Kahn K, Keeler WB, Ruhenstein LV, Kosecoff J and Brook RH, Quality of care before and after implementation of the DRG-based prospective payment systcm, The Journal of the American Medical Association 1990;264:15:1989-1994.

- Rosko MD and Brogles RW, The impact of the New Jersey all-payer DRG-system, Inquiry 1986;23:65-75.

- Rosko MD and Brogles RW, Short term responses of hospitals to the DRG prospective pricing mechanism in New Jersey, Medical Care 1987;25:2:88-99.

- Ruchlin HS and Leveson I. Measuring hospital productivity, Health Services Research 1974:308-323.

- Sawitz E, Showstack JA. Chow J and Schroeder SA. The use of inhospital physician services for Acute Myocardial Infarction; changes in volume and complexity over time, The Journal of the American Medical Association 1988; 259:16:2419-2422.

- Schelling. AN. Kostprijsonderzoek op de behandelkamer Gastro-Enterologie Maastricht, Master's thesis Bedrijiskunde TUE, 1988.

- Schroeder LD. Sjoquist DL and Siephan PE, Understanding regression analysis; an introductory guide. Sage university paper, number 07-057, Beverly Hills, 1986.

Schumacher DN, Chan C. Medical staff and hospital administration uses of discharge abstract data. Quality Review Bulletin 1981:7:6:26-35.

- Simborg DW, DRG-creep; a new hospital-acquired disease. The New England Journal of Medicine 1981:304:26:1602-1604.

- Slabbers AML. Kostprijsonderzoek op de ondersteunende afdeling Klinische Neurofysiologie. Master's thesis Bedrijfskunde. TUE, 1987.

- Smis HL. Fetter RB and McHahon LF, Variation in resource use within diagnosis related groups: the severity issue, Health Care Financing Review 1984:71-78.

- Stcenbakkers WHM. Een kostprijsonderzoek op de medisch ondersteunende afdeling der fisiotherapie. Masicr's thesis HTS. Eindhoven, 1988.

- Stein RE, Gortmaker SL, Perrin EC, Perrin JM, Pless IB, Walker DK and Weitzman M, Severity of illness: concepts and measurements. The Lancet 1987;1506-1509.

- Steingard RM. e.a.. Sex differences in the management of coronary artery disease, The New England Journal of Medicine 1991:325:4:226-230.

- Stern RS. Epstein AM, Institutional responses to prospective payment based on diagnosis related groups. The New England Journal of Medicine 1985;312:10:621-627. 
- The Pittsburgh Research Institute, Patients Management Categories. Release 3.0. Blue Cross of Western Pennsylvania and Maryland Blue Cross version, 1988.

- Thomas JW, Ashcraft MLF, and Zimmerman J, An evaluation of alternative severity of illness measures for use by university hospitals, volume 1: Management Summary. Department of Health Services Management and Policy. The University of Michigan. Ann Arbor, Michigan, 1986.

- Thomas JW, Ashcraft MLF and Zimmerman J, An evaluation of alternative severity of illness measures for use by university hospitals, volume 2: Technical Report, Department of Health Services Management and Policy, The University of Michigan, Ann Aibor, Michigan, 1986.

- Thomas JW, Ashcraft MLF and Zimmerman J, An evaluation of alternative severity of illness measures for use by university hospitals, volume 3: Appendices, Department. of Health Services Management and Policy, The University of Michigan, Ann Arbor, Michigan, 1986.

- Thomas JW and Asheraft ML, Measuring severity of illness: a comparison of interrater reliability among severity methodologies, Inquiry 1989;26:483-492.

- Thomas JW and Ashcraft ML. Measuring severity of illness: six severity systems and their ability of explain cost variation. Inquiry 1991;28:39-55.

- Torfs K, DRG-financiering in de V.S.; ecrste evaluatie van financiéle en economische resultaten, Acta Hospitalia 1989;1:7-29.

- Torfs K, DRG-financiering voor ziekenhuizen; met bedoelde effecten. Acta Hospitalia 1989;2:5-21.

- Ven v.d. WPMM, Nauta A, Vliet v. RCJA and Rutten FFH, Inventarisatie en achtergronden van de consumptie verschillen tussen ziekenfonds- en particulierverzekerden, Gezondheid en Samenleving 1980;1:4:224-254.

- Voss GBWE, Patient-classificatie systemen in de Verenigde Staten, Tijdschrift Sociale Gezondheidszorg 1987;65:12:392-402.

- Voss GBWE en Silkens RMJ, Patiënt-gerelateerde kosteninformatic: ontwikkeling van een DRG-patiëntclassificatiesysteem in het academisch ziekenhuis Maastricht, Tijdschrift Medische Informarica 1988;17:2:61-65.

- Voss G, Dijk van P, DRG's: onbekend maakt onbemind. Medisch Contact 1988;12:365. 368.

- Wagner DP and Draper EA, Acute physiology and chronic health evaluation (APACHE II) and medicare reimburscment, Health Care Financing Review 1984;91-105.

- Watts CA and Klastorin TD. The impact of case-mix on hospitals costs; a comparative analysis, Inquiry 1980;17:357-367.

- Weinstein MC, Coxson PG. Williams LW. Pass TM, Stason WB and Goldman L, Forecasting coronary heart disease incidence mortality and cost: the coronary heart disease policy model, American Journal of Public Health 1987;77:11:1417-1426.

- Wenemoser RAS. Kostprijscalculatie op de ondersteunende afdeling Nucleaire Geneeskunde, Master's thesis HEAO, Sittard, 1988.

- West H, McMenamin MP and Guralnick L, Physician and hospital reimbursement study, report, HHS Contract No. 100-83-0026 Mandex, Inc., Vienna, Virginia, 1985. In: Review on the literature on Diagnosis Related Groups by Worthman LG, Cretin S, RAND, 1986.

- Williams SV, Finkler SA, Murphy CM and Eisenberg JM, Improved cost allocation in case-mix accounting, Medical Care 1982;20:5:450-459.

- Williams SV, Kominsky GF, Dowd BE and Soper KA, Methodological limitations in case-mix hospital reimbursement, with a proposal for change, Inquiry 1984;21:17-31.

- Willems JL, e.a. (cooperative study), Criteria for intraventricular conduction disturbances and pre-excitation, Journal of the American College of Cardiology 1985;5:6:1261-1275.

- Wood WR, Ament RP and Kobrinski EG, A foundation for hospital case-mix measurement, Inquiry 1981;18:247-254.

- Worthman LG and Cretin S, Review of the literature on Diagnosis Related Croups, Rand Note, n-2492-HCFA, 1986.

- Wyszewianski L. Thomas JW and Friedman BA, Case-based payment and the control of quality and efficiency in hospitals, Inquiry 1987;24:17-25.

- Young WW, Swinkola RB and Zorn DM, The measurement of hospital case-mix, Medical Care 1982;20:5:501-512. 
- Young DW and Saltman RB, Prospective reimbursement and the hospital power equilibrium: a-matrix-based management control system, Inquiry 1983:20:20-23.

- Young WW, Incorporating severity of illness and comorbidity in case-mix measurement, Health Care Financing Review 1984;23-31.

- Young WW, Linking financial analysis and quality assurance using patient management categories, Journal of Quality Assurance 1984;17:1.7-21.

- Young WW, Schuchert JL and Patterson MS, ICD-9-cm code and coding practice changes for case-mix measurement, Journal of the American Medical Record Association 1986;29-33.

- Zwaan de C, Willems GM, Vermeer F, Res J, Verheugt FWA, Laarse van den A, Simoons ML, Lubsen J. Hermans WTh, Enzyme test in the evaluation of thrombolysis in acute. myocardial infarction, British Heart Journal 1988;59:175-183. 


\section{Appendices}

Appendix 1

Appendix 2

Appendix 3

Appendix 4

Appendix 5

Appendix 6

Appendix 7

Appendix 8

Appendix 9

Appendix 10

Appendix II

Appendix 12

Appendix 13

Appendix 14

Appendix 15

Appendix 16

Appendix 17

Appendix 18

Appendix 19

Appendix 20

Appendix 21

Appendix 22

Appendix 23

Appendix 24

Appendix 25
Data registration form for time period $\mathrm{Tl}$.

Descriptive Statistics of Costs of Laboratory Services per Time Period and for the Total Hospital Stay.

Descriptive Statistics of Costs of ECG per Time Period and for the Total Hospital Stay.

Descriptive Statistics of Costs of Radiology Tests per Time Period and for the Total Hospital Stay.

Descriptive Statistics of Costs of Heart Catheterization per Time Period and for the Total Hospital Stay.

Descriptive Statistics of Costs of Exercise ECG per Time Period and for the Total Hospital Stay.

Descriptive Statistics of Costs of Holter Electrocardiogram per Time Period and for the Total Hospital Stay.

Descriptive Statistics of Costs of Echocardioghrapy per Time Period and for the Total Hospital Stay.

Descriptive Statistics of Costs of Thallium Tests per Time Period and for the Total Hospital Stay.

Descriptive Statistics of Costs of Thrombolytic Therapy per Time Period and for the Total Hospital Stay.

Descriptive Statistics of Costs of Drugs (except thrombolytic therapy) per Time Period and for the Total Hospital Stay.

Descriptive Statistics of Costs of PTCA per Time Period and for the Total Hospital Stay.

Descriptive Statistics of Costs of Other Medical Interventions per Time Period and for the Total Hospital Stay.

Descriptive Statistics of Costs of Intensive Care per Time Period and for the Total Hospital Stay.

Descriptive Statistics of Costs of Physical Therapy per Time Period and for the Total Hospital Stay.

Severity Dummy variables.

Correlation matrix concerning severity of illness on admission. background characteristics and costs of thrombolytic therapy.

Correlation matrix of severity of illness on admission, medical treatment, background characteristics and costs of heart catheterization during period To.

Correlation matrix of severity of illness on admission, medical treatment and costs of PTCA for all catheterized AMI patients.

Correlation matrix of severity of illness indicators, background yariables and length of stay.

Correlation matrix of severity of illness indicators, background variables, length of stay and costs of $\mathrm{CCU}$.

Mean costs of drugs of patients in severity categories representing ischemic pain.

Mean costs of drugs of patients in severity categories representing the extent of Cardiac Wall Motion Abnormalities.

Mean costs of drugs of patients in severity categories representing type and time of appearance of cardiac rhythm disorders.

Mean costs of drugs of patients in severity categories representing the Left Ventricular. Ejection Fraction. 
Appendix 26

Appendix 27

Appendix 28

Appendix 29

Appendix 30

Appendix 31

Appendix 32

Appendix 33

Appendix 34

Appendix 35

Appendix 36

Appendix 37

Appendix 38

Appendix 39

Appendix 40

Appendix 41

Appendix 42

Appendix 43

Appendix 44

Appendix 45

Appendix 46

Appendix 47

Appendix 48

Appendix 49

Appendix 50
Correlation matrix of severity of illness indicators, background variables, length of stay and costs of drugs.

Results from regression analysis: dependent variable is total costs of drugs in AMI-patients.

Mean costs of laboratory services of patients in severity categories representing myocardial necrosis estimated by total CPK release.

Mean costs of laboratory services of patients in severity categories representing ischemic pain.

Mean costs of laboratory services of patients in severity categories representing the extent of Cardiac Wall Motion Abnormalities.

Mean costs of laboratory services of patients in severity categories representing type and time of appearance of cardiac rhythm disorders.

Correlations matrix of severity of illness indicators, background variables, length of stay, enrollment in PRIMl protocol and costs of laboratory services.

Results from regression analysis: dependent variable is total costs of laboratory services.

Mean costs of ECG of patients in severity categories representing myocardial necrosis estimated by total CPK release.

Mean costs of ECG of patients in severity categories representing ischemic pain.

Mean costs of ECG of patients in severity categories representing the extent of Cardiac Wall Motion Abnormalities.

Mean costs of ECG of patients in severity cateogries representing type and time of appearance of cardiac rhythm disorders.

Mean costs of ECG of patients in severity categories representing Left Ventricular Ejection Fraction.

Correlation matrix of severily of illness indicators, background variables, length of stay and costs of ECG.

Results from regression analysis: dependent variable is costs of ECG. Mean costs of echocardiography of patients in severity categories representing myocardial necrosis estimated by total CPK release.

Mean costs of echocardiography of patients in severity catcgories representing type and time of appearance of cardiac rhythm disorders.

Correlation matrix of severity of illness indicators, background variables, length of stay and costs of echocurdiography.

Results from regression analysis: dependent variable is costs of echocardiography.

Mean costs of exercise tests of patients in severity categories representing by lype and time appearance of cardiac rhythm disorders.

Mean costs of exercise tests of patients in severity categories representing the Left Ventricular Ejection Fraction.

Correlation matrix of severily of illness indicators, background variables, length of stay and costs of exercise tests.

Results from regression analysis: dependent variable is costs of exercise ECG.

Pattern-analysis.

Means, standard deviations and numbers of the SICLOS for AMIpatients by age and sex. 
Appendix 1 Data registration form for time period $\mathrm{T} 1$.

REGISTKATIEFORMULIEIR ACUUT MYOCARD INFARCT VOORGESCTUEDENIS

ELSICONDICATOREN U POSIIE)

RFAM

RROK

ROVERIG

\section{YOOROESCIIIEDENS}

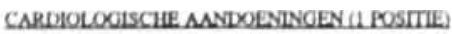
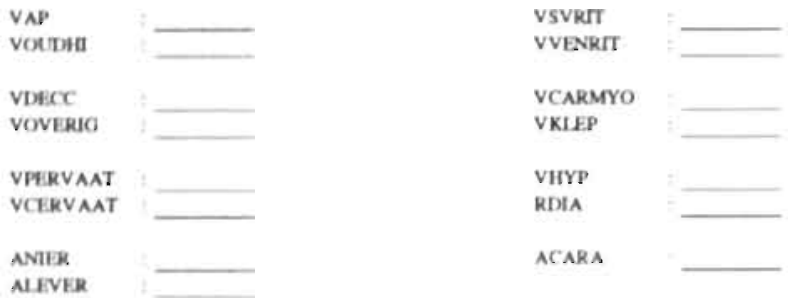

VAVGEL.

VTVGEL.

VCONG

AOVERIG

RVET

vCOR.

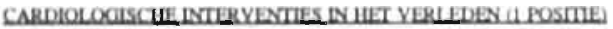

VPACE

VTROM

\section{CONTRANDICATHES C ROSHIE)}

VREOPER

VRECAT

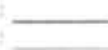

VREMANG

VHOSTEN

REGISTRATIFFORMULIFR ACITT MYOCARD INFARCT PFRIODE T. 1

\section{ANAMENESE (1 POSME}

PYNI

(2 POS)

PAI.I

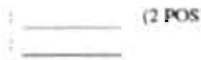

ONDERZOEX LNCHAMELUS U POSTIE

OEDEMI

BLDRHI

BLDRL!

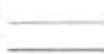

LONGS

LEVGR!

BORIYY

PRCOL.
sycot.

APKL!

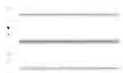

DYSPN:

WNDERZOEKECG

RMMESTOOBNHSEN

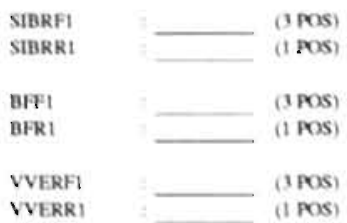

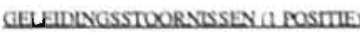

IGRAV I

KCRAR:

Morv1

MOTR I

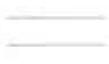

3 POS

(1) POS:

NSVTFI

(3) pos:

(1) POS:

(1) POS)

NERFII
MOTVI

MOtrR । 
HEMTI

HEMER !

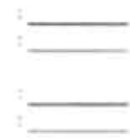

BFBV

BPBR1

LOKALISATE NECROSE U.POSIIE

NESEPI

NEANT1

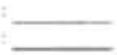

LOKALISATIE ISCHEMIE UROSTIE

APSEP1

APANTI

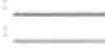

COMPLICATIES U POSTHE

COMCAI

COFER 1

COCVAI

COMOH!

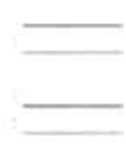

LBTBV

LBTBRI

THeV!

THERI

NE $A T$

NEPOS!

AM.AT

APros!

APNT.

ANEVI

COMMII

comact

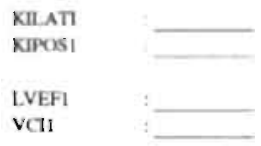

KIINFI

KIBAS I

EDHVD1 (2 POS)

\section{CAG (3 POSWES)}

RCAl

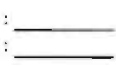

$\mathrm{RCX}$ :

[US!

BRLCEPROTOKOL L LOSTIVE

DSEPI

DI.ATI

NANTI

[NPOS]

ININFI

BRUCEI

THALLUMUPOSTIE

THSEPI

THANTI

TIII-ATI

TIPOS 1

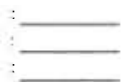

TINF]

THREVI

THBASI

SWAN-GANZ U POSME

SWG!

\section{DIERVENTIES U POSTIIE}

TROMI

(2 POS)

[RESU]

[PACT]

IPACTI

IPTCA]

\section{LABORATORIMM (4 BOSUTESI}

SGPTW!

Lorw'

SGOTW!

SGOTT!

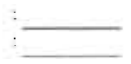

(3 POS)

CPKW!

CPKTI

MEDICATIE O POSLUES

[BETA!

[NTR]

ICAAN!
WIUR!

IGLYC.

LANARI
IANHY!

IANCOI

[ACET]

IANBIR: 
Appendix 2 Descriptive Statistics of Costs of Laboratory Services per Time Period and for the Total Hospital Stay.

\begin{tabular}{lrrrrr}
\hline & $\begin{array}{r}\text { Total of } \\
\text { Services }\end{array}$ & $\begin{array}{r}\text { Total } \\
\text { Costs }\end{array}$ & $\begin{array}{r}\text { Number of Valid } \\
\text { Cases }\end{array}$ & $\begin{array}{r}\text { Mean } \\
\text { Costs }\end{array}$ & $\begin{array}{r}\text { Sid. } \\
\text { Dev. }\end{array}$ \\
Time Period T0 & 3812 & $12,335.80$ & 443 & 27.85 & 12.65 \\
Time Period T1 & 24102 & $62,744.32$ & 439 & 142.93 & 109.69 \\
Time Period T2 & 6535 & $18,021.13$ & 419 & 43.01 & 78.75 \\
Time Period T3 & 2765 & $8,209.91$ & 173 & 47.46 & 113.03 \\
Total Hospital Sialy & 37214 & $101,310.34$ & 464 & 218.34 & $\mathbf{1 8 3 . 1 6}$ \\
\hline
\end{tabular}

Appendix 3 Descriptive Statistics of Costs of ECG per Time Period and for the Total Hospital Stay.

\begin{tabular}{lrrrrr}
\hline & $\begin{array}{r}\text { Total of } \\
\text { Services }\end{array}$ & $\begin{array}{r}\text { Total } \\
\text { Costs }\end{array}$ & $\begin{array}{r}\text { Number of Valid } \\
\text { Cases }\end{array}$ & $\begin{array}{r}\text { Mean } \\
\text { Costs }\end{array}$ & $\begin{array}{r}\text { Sid. } \\
\text { Dev. }\end{array}$ \\
& & & & & \\
Time Period T $0+T 1$ & 437 & $2,779.32$ & 439 & 6.33 & 6.35 \\
Time Period T2 & 2075 & 13.197 .00 & 419 & 31.50 & 13.60 \\
Time Period T3 & 980 & 6.232 .80 & 173 & 36.03 & 49.60 \\
Total Hospital Stay & 3492 & $22,209.12$ & 464 & $\mathbf{4 7 . 8 6}$ & $\mathbf{4 2 . 6 0}$ \\
\hline
\end{tabular}

Appendix 4 Descriptive Statistics of Costs of Radiology Tests per Time Period and for the Total Hospital Stay.

\begin{tabular}{lrrrrr}
\hline & $\begin{array}{r}\text { Total of } \\
\text { Senvices }\end{array}$ & $\begin{array}{r}\text { Total } \\
\text { Costs }\end{array}$ & $\begin{array}{r}\text { Number of Valid } \\
\text { Cases }\end{array}$ & $\begin{array}{r}\text { Mean } \\
\text { Costs }\end{array}$ & $\begin{array}{r}\text { Sid. } \\
\text { Dev. }\end{array}$ \\
Time Period T0+TI & 558 & $12,419.80$ & 439 & 28.29 & 20.57 \\
Time Period 72 & 291 & 0.424 .00 & 419 & 15.33 & 19.06 \\
Time Period T3 & 113 & $2,552.60$ & 173 & 14.75 & 36.65 \\
Total Hospital Stay & 962 & 21.396 .40 & 464 & $46.1 !$ & 46.17 \\
\hline
\end{tabular}


Appendix 5 Descriptive Statistics of Costs of Heart Catheterization per Time Period and for the Total Hospital Stay.

\begin{tabular}{lrrrrr} 
& $\begin{array}{r}\text { Total of } \\
\text { Services }\end{array}$ & $\begin{array}{r}\text { Total } \\
\text { Costs }\end{array}$ & $\begin{array}{r}\text { Number of Valid } \\
\text { Cases }\end{array}$ & $\begin{array}{r}\text { Mean } \\
\text { Costs }\end{array}$ & $\begin{array}{r}\text { Std. } \\
\text { Dev }\end{array}$ \\
& & & & & \\
Time Period T0 & 467 & $144,501.53$ & 443 & 326.19 & 452.91 \\
Time Period T1 & 251 & $78,822.31$ & 439 & 179.55 & 388.28 \\
Time Period T2 & 98 & $31,272.21$ & 419 & 74.64 & 264.96 \\
Time Period T3 & 41 & $15,016.49$ & 173 & 86.80 & 257.55 \\
Total Hospital Stay & 857 & 269.612 .54 & 464 & 581.06 & 750.71 \\
\hline
\end{tabular}

Appendix 6 Descriptive Statistics of Costs of Exercise ECG per Time Period and for the Total Hospital Stay.

\begin{tabular}{lrrrrr} 
& $\begin{array}{r}\text { Total of } \\
\text { Services }\end{array}$ & $\begin{array}{r}\text { Total } \\
\text { Costs }\end{array}$ & $\begin{array}{r}\text { Number of Valid } \\
\text { Cases }\end{array}$ & $\begin{array}{r}\text { Mean } \\
\text { Costs }\end{array}$ & $\begin{array}{r}\text { Std. } \\
\text { Dev. }\end{array}$ \\
& & & & & \\
Time Period TO & 1 & 38.75 & 443 & 0.09 & 1.84 \\
Time Period TI & 3 & 116.25 & 439 & 0.26 & 3.20 \\
Time Period T2 & 139 & $5,386.25$ & 179 & 12.86 & 18.66 \\
Time Period T3 & 63 & $2,441.25$ & 173 & 14.11 & 20.05 \\
Total Hospital Stay & 206 & $7,982.50$ & 464 & 17.20 & 20.58 \\
\hline
\end{tabular}

Appendix 7 Descriptive Statistics of Costs of Holter Electrocardiogram per Time Period and for the Total Hospital Stay.

\begin{tabular}{lrrrrr}
\hline & $\begin{array}{r}\text { Total of } \\
\text { Services }\end{array}$ & $\begin{array}{r}\text { Total } \\
\text { Costs }\end{array}$ & $\begin{array}{r}\text { Number of Valid } \\
\text { Cases }\end{array}$ & $\begin{array}{r}\text { Mean } \\
\text { Costs }\end{array}$ & $\begin{array}{c}\text { Sid. } \\
\text { Dev. }\end{array}$ \\
Time Period To & 0 & 0 & 443 & 0.00 & - \\
Time Period T1 & 23 & $1,248.90$ & 439 & 2.84 & 12.66 \\
Time Period T2 & 33 & $1,791.90$ & 419 & 4.28 & 16.02 \\
Time Period T3 & 16 & 868.80 & 173 & 5.02 & 28.24 \\
Total Hospital Stay & 72 & $3,909.60$ & 464 & 8.43 & 25.34 \\
\hline
\end{tabular}


Appendix 8 Descriptive Statistics of Costs of Echocardioghrapy per Time Period and for the Total Hospital Stay.

\begin{tabular}{lrrrrr}
\hline & $\begin{array}{c}\text { Total of } \\
\text { Services }\end{array}$ & $\begin{array}{r}\text { Total } \\
\text { Costs }\end{array}$ & $\begin{array}{r}\text { Number of Valid } \\
\text { Cases }\end{array}$ & $\begin{array}{r}\text { Mean } \\
\text { Costs }\end{array}$ & $\begin{array}{r}\text { Std. } \\
\text { Dev. }\end{array}$ \\
Time Period TO & 18 & 819.70 & 443 & 1.85 & 11.18 \\
Time Period T1 & 104 & $5,046.80$ & 439 & 11.50 & 23.56 \\
Time Period T2 & 253 & $12,409.65$ & 419 & 29.62 & 28.40 \\
Time Period T3 & 42 & $1,924.90$ & 173 & 11.13 & 26.51 \\
Total Hospital Stay & 417 & $20,201.05$ & 464 & $\mathbf{4 3 . 5 4}$ & 32.76 \\
\hline
\end{tabular}

Appendix 9 Descriptive Statistics of Costs of Thallium Tests per Time Period and for the Total Hospital Stay.

\begin{tabular}{lrrrrr}
\hline & $\begin{array}{r}\text { Total of } \\
\text { Services }\end{array}$ & $\begin{array}{r}\text { Total } \\
\text { Costs }\end{array}$ & Number of Valid & Mean & Sid. \\
Cases & Costs & Dev. \\
Time Period T0 & 1 & 72.52 & & & \\
Time Period TI & 46 & $3,276.91$ & 443 & 0.16 & 3.45 \\
Time Period T2 & 147 & $10,406.52$ & 439 & 7.46 & 32.76 \\
Time Period T3 & 41 & $3,444.73$ & 419 & 24.84 & 54.10 \\
& & & 173 & 19.91 & 64.93 \\
Total Hospital Stay & 235 & $17,200.68$ & 464 & 37.07 & 71.18 \\
\hline
\end{tabular}

Appendix 10 Descriptive Statistics of Costs of Thrombolytic Therapy per Time Period and for the Total Hospital Stay.

\begin{tabular}{|c|c|c|c|}
\hline $\begin{array}{l}\text { Total of } \\
\text { Services }\end{array}$ & $\begin{array}{l}\text { Total } \\
\text { Costs }\end{array}$ & $\begin{array}{r}\text { Number of Valid } \\
\text { Cases }\end{array}$ & $\begin{array}{l}\text { Mean } \\
\text { Costs }\end{array}$ \\
\hline
\end{tabular}

$\begin{array}{lrrrrr}\text { Time Period T0 } & 145 & 80.844 .75 & 442 & 182.91 & 3.66 \\ \text { Time Period TI } & 3 & 1.672 .65 & 438 & 3.82 & \mathbf{4 6 . 0 4} \\ \text { Time Period T2 } & 2 & 1.115 .10 & 419 & 2.66 & 38.47 \\ \text { Time Period T3 } & 0 & 0.00 & 173 & 0.00 & - \\ & & & & & \\ \text { Total Hospital Stay } & 150 & 83.632 .50 & 464 & 180.24 & \mathbf{2 8 0 . 8 8}\end{array}$


Appendix 11 Descriptive Statistics of Costs of Drugs (except thrombolytic therapy) per Time Period and for the Total Hospital Stay.

\begin{tabular}{lrrrrr} 
& $\begin{array}{r}\text { Total of } \\
\text { Services }\end{array}$ & $\begin{array}{r}\text { Total } \\
\text { Costs }\end{array}$ & $\begin{array}{r}\text { Number of Valid } \\
\text { Cases }\end{array}$ & $\begin{array}{r}\text { Mean } \\
\text { Costs }\end{array}$ & $\begin{array}{r}\text { Std. } \\
\text { Dev. }\end{array}$ \\
& & & & & \\
Time Period T0 & 1564 & 3.533 .00 & 441 & 8.01 & 3.66 \\
Time Period T1 & 3252 & $6,384.65$ & 4138 & 14.58 & 7.61 \\
Time Period T2 & 7832 & $14,206.65$ & 419 & 33.91 & 19.72 \\
Time Period T3 & 5061 & $9,099.10$ & 173 & 52.60 & 96.49 \\
Total Hospital Stay & 17709 & 33.223 .40 & 464 & 71.60 & 79.14 \\
\hline
\end{tabular}

Appendix 12 Descriptive Statistics of Costs of PTCA per Time Period and for the Total Hospital Stay.

\begin{tabular}{|c|c|c|c|}
\hline $\begin{array}{l}\text { Total of } \\
\text { Services }\end{array}$ & $\begin{array}{l}\text { Total } \\
\text { Costs }\end{array}$ & $\begin{array}{r}\text { Namber of Valid } \\
\text { Cases }\end{array}$ & $\begin{array}{l}\text { Mean } \\
\text { Costs }\end{array}$ \\
\hline
\end{tabular}

\begin{tabular}{lrrrrr} 
Time Period TO & 50 & $183,862.00$ & 435 & 422.67 & 1174.22 \\
Time Period T1 & 19 & $69,867.56$ & 439 & 161.73 & 754.91 \\
Time Period T2 & 10 & $36,772.40$ & 419 & 87.76 & 561.94 \\
Time Period T3 & 3 & 11.031 .72 & 173 & 63.77 & 481.42 \\
& & & & & \\
Total Hospital Stay & 82 & $301,533.68$ & 464 & 649.86 & 1424.79 \\
\hline
\end{tabular}

Appendix 13 Descriptive Statistics of Costs of Other Medical Interventions per Time Period and for the Total Hospital Stay.

\begin{tabular}{lrrrrr} 
& $\begin{array}{r}\text { Total of } \\
\text { Services }\end{array}$ & $\begin{array}{r}\text { Total } \\
\text { Costs }\end{array}$ & Number of Valid & Mian & Sid. \\
& & & Cases & Costs & Dev. \\
Time Period To & 32 & $4,032.60$ & 442 & 9.12 & 112.16 \\
Time Period T1 & 34 & $9,481.22$ & 439 & 21.60 & 177.89 \\
Time Period T2 & 15 & 507.50 & 419 & 1.21 & 9.23 \\
Time Period T3 & 14 & 610.12 & 173 & 3.53 & 20.77 \\
Total Hospital Stay & 95 & 14.631 .44 & 464 & 3153 & 257.26 \\
\hline
\end{tabular}


Appendix 14 Descriptive Statistics of Costs of Intensive Care per Time Period and for the Total Hospital Stay.

\begin{tabular}{lrrrrr}
\hline & $\begin{array}{r}\text { Total of } \\
\text { Services }\end{array}$ & $\begin{array}{r}\text { Total } \\
\text { Costs }\end{array}$ & $\begin{array}{r}\text { Number of Valid } \\
\text { Cases }\end{array}$ & $\begin{array}{r}\text { Mean } \\
\text { Costs }\end{array}$ & $\begin{array}{r}\text { Std. } \\
\text { Dev. }\end{array}$ \\
& & & & & \\
Time Period TI & 1120 & $324,688.00$ & 439 & 739.61 & 223.61 \\
Time Period T2 & 705 & $204,379.50$ & 419 & 487.78 & 683.97 \\
Time Period T3 & 403 & $116,829.70$ & 173 & 675.32 & 1535.00 \\
Total Hospital Stay & 2228 & $645,897.20$ & 464 & 1392.02 & 1577.40 \\
\hline
\end{tabular}

Appendix 15 Descriptive Statistics of Costs of Physical Therapy per Time Period and for the Total Hospital Stay.

\begin{tabular}{|c|c|c|c|c|c|}
\hline & $\begin{array}{l}\text { Total of } \\
\text { Services }\end{array}$ & $\begin{array}{l}\text { Total } \\
\text { Costs }\end{array}$ & $\begin{array}{r}\text { Number of Valid } \\
\text { Cases }\end{array}$ & $\begin{array}{l}\text { Mean } \\
\text { Costs }\end{array}$ & $\begin{array}{l}\text { Std. } \\
\text { Dev. }\end{array}$ \\
\hline Time Period $T 0+T l$ & 497 & $4,835.18$ & 439 & 11.01 & 21.83 \\
\hline Time Period T2 & 484 & $5,100.24$ & 419 & 12.07 & 22.07 \\
\hline Time Parind T? & 1.27 & 1.20 .1 .51 & 172 & 7.18 & 15.72 \\
\hline Total Hospinal Siay & 1118 & 11.176 .93 & 464 & 24.09 & 30.32 \\
\hline
\end{tabular}

Appendix 16 Severily Dummy variables.

Severity of Coronary stenosis:

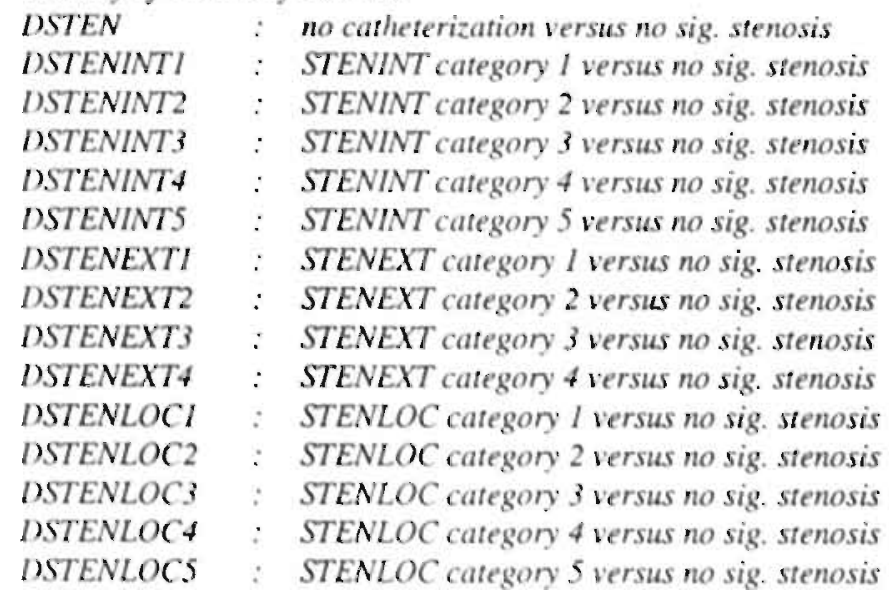

Severity of Myocandial Ischemia:

DBRUCE : no bruce tes? versus no ischemia

DBRUCINTI : BRUCINT category l versus no ischemia 


\begin{tabular}{|c|c|c|}
\hline DBRUCINT2 & $:$ & BRUCINT category 2 versus no ischemia \\
\hline DBRUCINT3 & : & BRUCINT category 3 versus no ischemia \\
\hline DBRUCINT4 & : & BRUCINT category 4 versus no ischemia \\
\hline BRUCINTS & $:$ & BRUCINT category 5 versus no ischemia \\
\hline BRUCEXTI & : & BRUCEXT category I versus no ischemia \\
\hline DBRUCEXT2 & : & BRUCEXT category 2 versus no ischemia \\
\hline BRUCEXT3 & $:$ & BRUCEXT category 3 versus no ischemia \\
\hline DBRUCEXT4 & : & BRUCEXT category 4 versus no ischemia \\
\hline DBRUCEXT5 & : & BRUCEXT category 5 versus no ischemia \\
\hline DBRUCLOCI & : & BRUCLOC category 1 versus no ischemia \\
\hline DBRUCLOC2 & $:$ & BRUCLOC category 2 versus no ischemia \\
\hline DBRUCEXPI & : & BRUCEXP category I versus no ischemia \\
\hline$B R U C E X P 2$ & : & BRUCEXP category 2 versus no ischemia \\
\hline DBRUCEXP3 & : & BRUCEXP category 3 versus no ischemia \\
\hline BRUCEXP4 & $\therefore$ & BRUCEXP category 4 versus no ischemia \\
\hline THAL & & no thallium test versus no rev. thallium changes \\
\hline ISTHEXTI & $:$ & ISTHEXT category I versus no rev. thallium changes \\
\hline ISTHLOCI & & ISTHLOC category I versus no rev. thallium changes \\
\hline
\end{tabular}

Severity of Myocandial Necrosis:

DCPK
DCPKI
DCPK2
DCPK3
DCPK4
DCPK5
DSGOT
DSGOTI
DSGOT2
DSGOT3
DSGOT4
DSGOT5
DSGOT6
DLDH
DLDHI
DLDH 2
DLDH3
DLDH4
DLDH5
DNETHEXTI
DNETHEXT2
DNETHLOCI

: no CPK-area measure versus no $C P K$ changes

: $\quad C P K$ category 1 versus no $C P K$ changes

- $\quad C P K$ category 2 versus no $C P K$ changes

: $\quad C P K$ category 3 versus no $C P K$ chamges

: $\quad C P K$ category 4 versus no $C P K$ changes

- CPK category 5 versus no CPK changes

no SGOT-area measure versus no SGOT changes

- SGOT category 1 versus no SGOT changes

- SGOT category 2 versus no SGOT changes

- SGOT category 3 versus no SGOT changes

SGOT caregory 4 versus no SGOT changes

SGOT category 5 versus no SGOT changes

- SGOT category 6 versus no SGOT changes

- no LDH measure versus no LDH changes

LDH category 1 versus no $L D H$ changes

LDH category 2 versus no $L D H$ changes

- $L D H$ category 3 versus no $L D H$ changes

$\angle D H$ category 4 versus no $L D H$ changes

LDH category 5 versus no $L D H$ changes

NECTHEXT category / versus no irrev thallium changes

NECTHEXT category 2 versus no irrev. thallium changes

NECTHLOC category I versus no irrev. thallium changes

Severity of Ischemic Pain

DISPAIN : missing data versus no ischemic pain

DISPAINI : ISPAIN category I versus no ischemic pain

DISPAIN2 : ISPAIN category 2 versus no ischemic pain

Severity of Cardiac Wall Morion abnormalities

DECHO : no echocardiography versus no CWM abn.

DKIMIS : echocardiography, but no data versus no CWM abn. 


$\begin{array}{ll}\text { DKIINTI } & : \text { KIINT category } 1 \text { versus no CWM abn. } \\ \text { DKIEXTI } & : \text { KIEXT category I versus no CWM abn. } \\ \text { DKIEXT2 } & : \text { KIEXT category } 2 \text { versus no CWM abn. } \\ \text { DKIEXT3 } & : \text { KIEXT category } 3 \text { versus no CWM abn. } \\ \text { DKIEXT4 } & : \text { KIEXT category } 4 \text { versus no CWM abn. } \\ \text { DKIEXT5 } & : \text { KIEXT category } 5 \text { versus no CWM abn. } \\ \text { DKILOCI } & : \text { KILOC category } 1 \text { versus no CWM abn. }\end{array}$

Severity of Cardiac Rhylhm Disorders

$\begin{array}{lll}\text { DRHYTHMI } & : \text { RHYTHM category b versus no hythm disorders (a) } \\ \text { DRHYTHM2 } & : \text { RHYTHM category c versus no rhythm disorders (a) } \\ \text { DRHYTHM3 } & : \text { RHYTHM category d versus no rhythm disorders (a) } \\ \text { DRHYTHMA } & : \text { RHYTHM category e versus no rhythm disorders }(a) \\ \text { DRHYTHMS } & : \text { RHYTHM category f versus no rhythm disorders }(a) \\ \text { DRHYTHM6 } & : \text { RHYTHM category g versus no rhythm disorders }(a) \\ \text { DRHYTHM7 } & : \text { RHYTHM category } h \text { versus no rhythm disorders }(a) \\ \text { DRHYTHM8 } & : \text { RHYTHM category } i \text { versus no rhythm disorders }(a) \\ \text { DRHYTHMS } & : \text { RHYTHM category } j \text { versus no rhythm disorders }(a)\end{array}$

Severity of Cardiac Conducsion Disorders

DCONDUCI : CONDUC category b versus no conduction disorders $(a)$

DCONDUC2 : CONDUC category c versus no conduction disorders (a)

DCONDUC3 : CONDUC category d versus no conducrion disorders $(a)$

DCONDUCA : CONDUC category e versus no conduction disorders (a)

DCONDUC5 : CONDUC category fversus no conduction disorders $(a)$

DCONDUC6 : CONDUC category g versus no conduction disorders $(a)$

Severity of Heart Failure

DLVMIS : echocardiograpiny but no data LVEF versus no deviating LVEF

DLVEFI : LVEF category I versus no deviating $L V E F$

DLVEF2 : LVEF category 2 versus no deviating $L V E F$

DLVEF3 : LVEF category 3 versus no deviaing $L V E F$

Severity of Physical Consequences

$\begin{array}{ll}\text { DAP } & : \text { no AP classification versus no AP } \\ D A P 1 & : \text { AP category I versus no } A P \\ D A P 2 & : \text { AP category } 2 \text { versus no } A P \\ D A P 3 & : \text { AP category } 3 \text { versus no } A P\end{array}$

Admission Status:

DADINTO : regular admission in TO versus internal admission (already in hospital)

DADEXTI : regular admission in TO versus extemal admission in $T I$

DADEXT2 : regular admission in TO versus extemal admission in $T 2$

DADEXT3 : regular admission in TO versus esternal admission in $T 3$

Discharge Status:

DDEATHO : regular discharge in $T 2$ versus deceased in $T O$

DDEATHI : regular discharge in $T 2$ versus deceased in $T I$

DDEATH2 : regular discharge in $T 2$ versus deceased in $T 2$

DDEATH3 : regular discharge in $T 2$ versus deceased in $T 3$

DHOMEl : regular discharge in $T 2$ versus discharged to home in $T I$ 
DHOME3 : regular discharge in $T 2$ versus discharged to home in $T 3$

DTRANSI : regular discharge in T2 versus transferred to other medical speciality in $T I$

DTRANS2 : regular discharge in $T 2$ versus transferred to other medical speciality in $T 2$

DTRANS3 : regular discharge in $T 2$ versus transferred to other medical speciality in $T 3$

Appendix 17 Correlation matrix concerning severity of illness on admission, background characteristics and costs of thrombolytic therapy (Listwise deletion of missing cases, $n=254$ ).

\begin{tabular}{|c|c|c|c|c|c|c|c|c|c|}
\hline & CTHROMO & ESEXTO & ISLOCO & NECEXTO & NECL .000 & DEsfaENi & Dessañ & ABI & $\operatorname{six}$ \\
\hline ISEXTO & $.24^{*}$ & 1.00 & & & & & & & \\
\hline ISLOCO & $11 \%$ & $48^{\circ}$ & 1.00 & & & & & & \\
\hline NECEXTO0 & $.20^{\mathrm{m}}$ & $3 !^{\circ}$ & 08 & 1.00 & & & & & \\
\hline NECLOCO & $.19 n$ & $.21^{\circ}$ & $46^{\circ}$ & $67 *$ & 1.00 & & & & \\
\hline DISPAIN\| & $31^{*}$ & $.110^{*}$ & .07 & .07 & .05 & 1.00 & & & \\
\hline DISPAIN2 & $.26^{\circ}$ & $-12^{\circ}$ & .03 & .05. & .05 & .874 & 1.00 & & \\
\hline AGE & .06 & $-19^{*}$ & .02 & -02 & -.01 & -.03 & .09 & 1.00 & \\
\hline SEX & .08 & -07 & .02 & .08. & 03 & .10 & $16^{\mathrm{n}}$ & $.20^{\circ}$ & 1.00 \\
\hline
\end{tabular}

$p<.05$ 
Appendix 18 Correlation matrix of severity of illness on admission, medical treatment, background characteristics and costs of heart catheterization during period T0 (Listwise deletion of missing cases, $n=374$ ).

\begin{tabular}{|c|c|c|c|c|c|c|c|c|c|c|}
\hline & CCATO & IExTro & 18.0C: & NECEXTO & NBa.oco & THROMO & PTHROM & $A G$ & sex & DADINTTO \\
\hline ISEXTO & $19^{\circ}$ & 1.00 & & & & & & & & \\
\hline ISLOKCO & $14^{\circ}$ & $.49^{*}$ & 1.00 & & & & & & & \\
\hline NECEXTOO & $.12 "$ & $27 *$ & .07 & 1.00 & & & & & & \\
\hline NECLOCO & $.13{ }^{\circ}$ & $.14^{\circ}$ & $.45^{\circ}$ & $.66^{*}$ & 1.00 & & & & & \\
\hline THIROMO & $50^{4}$ & $.17 \bullet$ & .09 & $.15^{\circ}$ & $13^{*}$ & 1.00 & & & & \\
\hline FTHROM & $.12 *$ & $.04^{*}$ & .06 & .01 & .04 & .06 & 1.00 & & & \\
\hline$A G E$ & $-20^{\prime \prime}$ & $-14^{*}$ & -.04 & $-.15^{*}$ & .08 & .20 & .07 & 1.00 & & \\
\hline SEX & $.20^{4}$ & $.08 \bullet$ & .02 & $.13^{\circ}$ & .06 & $-.13^{\circ}$ & $.09 *$ & $.28^{*}$ & 1.00 & \\
\hline DADINTO & $.27 *$ & .03 & .02 & .05 & .06 & .02 & .04 & .05 & $-.20^{*}$ & 1.00 \\
\hline
\end{tabular}

$\bullet p<.05$

Appendix 19 Correlation matrix of severity of illness on admission, medical treatment and costs of PTCA for all catheterized AMI patients (Listwise deletion of missing cases, $n=127$ ).

\begin{tabular}{|c|c|c|c|c|c|c|c|c|}
\hline & TTCAN & THROMO & STENINO & STENEXO & STENLCO & NECT, 00 & INTER! & INTER2 \\
\hline THROMO & $-.21^{*}$ & 1.00 & & & & & & \\
\hline STININO & $.24^{\circ}$ & $-.24^{*}$ & 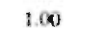 & & & & & \\
\hline STENIXX 0 & .10 & .08 & $81^{\circ}$ & 1.00 & & & & \\
\hline STENLO & .150 & .05 & $.69^{*}$ & $86^{\circ}$ & 1.00 & & & \\
\hline NECLOO &.$\infty$ & 22 & $14^{\circ}$ & $-18^{\circ}$ & $.0 \|$ & $1 . \infty$ & & \\
\hline INTERI & $31^{\circ}$ & -.08 & $.79 \bullet$ & $.70^{4}$ & 500 & $-.11^{*}$ & 1.00 & \\
\hline NTIE2 & .03 & $.7 !^{\bullet}$ & $.27 \bullet$ & $55^{\circ}$ & $49^{\circ}$ & .05 & $50^{\circ}$ & 1.00 \\
\hline
\end{tabular}

$\bullet p<05$ 
Appendix 20 Correlation matrix of severity of illness indicators, background variables and length of stay ( $L$ istwise deletion of missing cases, $n=451$ ).

\begin{tabular}{|c|c|}
\hline 关 & 8 \\
\hline ฐ & $8 \dot{8}$ \\
\hline$\overleftarrow{\imath}$ & 8 ह क व \\
\hline$\frac{1}{8}$ & 8 ₹ के में \\
\hline$\frac{\hat{E}}{2}$ & $8 \dot{8} 8 \div$ \\
\hline$\underline{\underline{z}}$ & $8 \div 388$ \\
\hline 啇 & 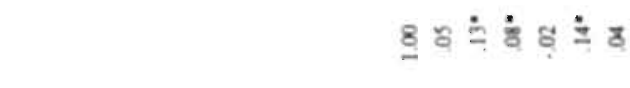 \\
\hline 产 & 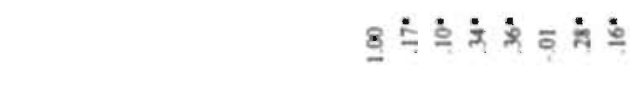 \\
\hline हृ & 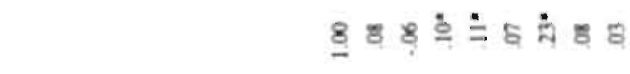 \\
\hline $\overrightarrow{\mathrm{g}}$ & $8 \dot{\overline{7}} 8$ वे $\dot{8} 88 \doteq \dot{0}$ \\
\hline 亳 & 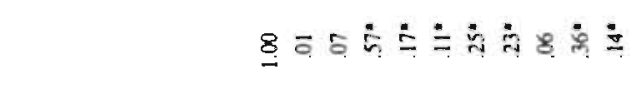 \\
\hline$\frac{a}{a}$ & 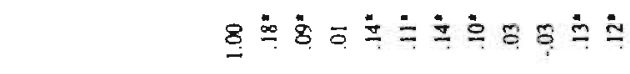 \\
\hline $\bar{I}$ & 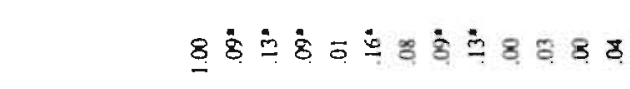 \\
\hline 䙷 & $8 \dot{9} \dot{9} \dot{9}$ 石 \\
\hline 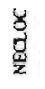 & $8 \%$ के \\
\hline te & 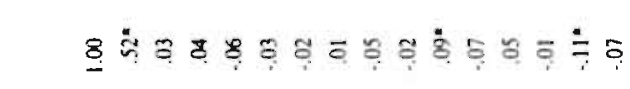 \\
\hline 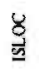 & 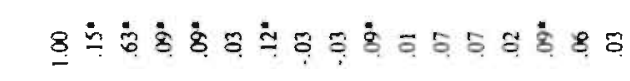 \\
\hline 意 & 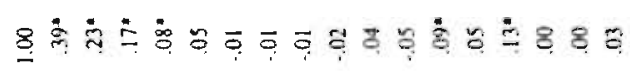 \\
\hline$\grave{s}$ & 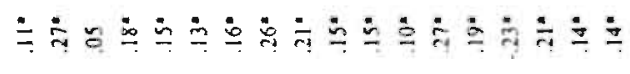 \\
\hline & 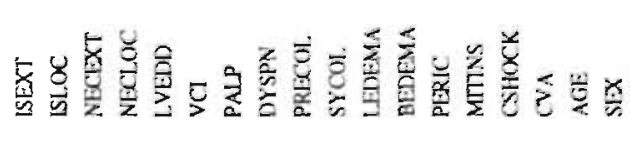 \\
\hline
\end{tabular}


Appendix 21 Correlation matrix of severity of illness indicators, background variables, length of stay and costs of CCU (Listwise deletion of missing cases, $n=454$ ).

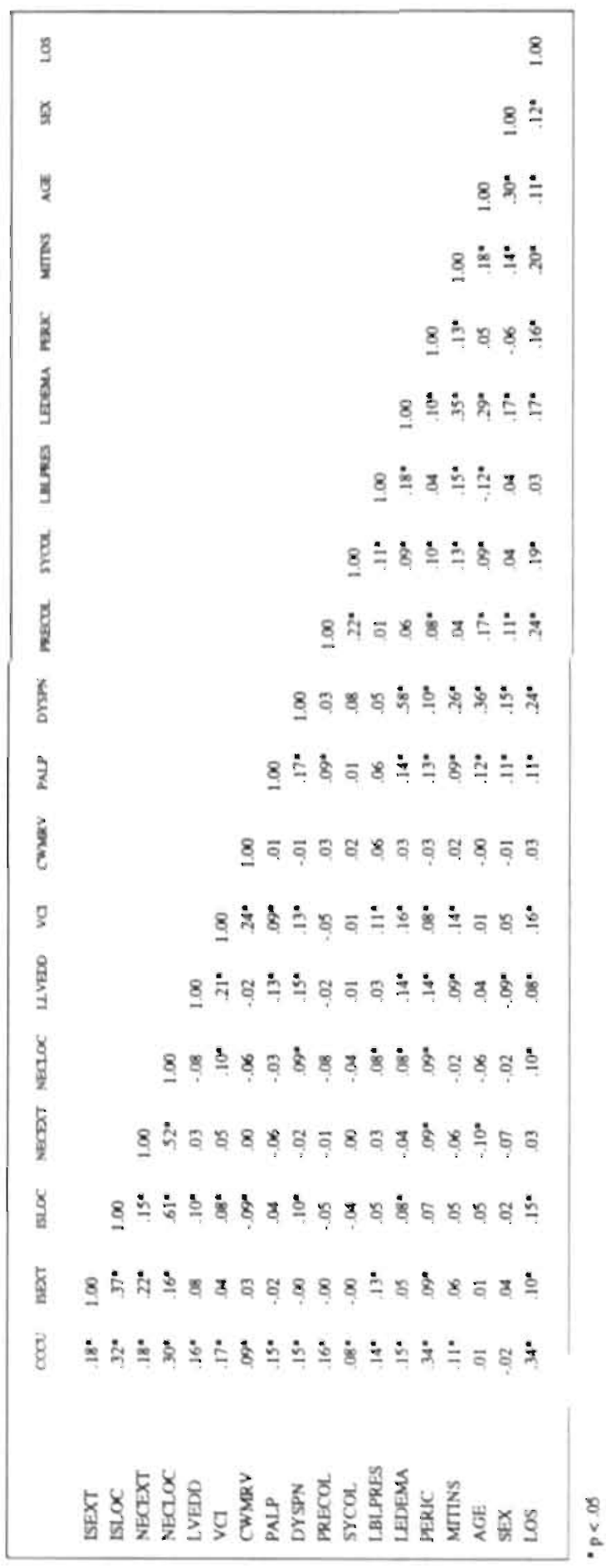


Appendix 22 Mean costs of drugs of patients in severity categories representing ischemic pain.

$\begin{array}{llrrr}\text { Category } & \text { Description } & \text { Mean } & \text { Std.dev. } & \text { Freq. } \\ & & & & \\ 0 & \text { no ischemic pain } & 38.93 & 53.79 & 54 \\ 1 & \text { ischemic pain in TO or Tl } & 60.67 & 42.36 & 304 \\ 2 & \text { ischemic pain (also) in T2 or T3 } & 105.78 & 73.67 & 92 \\ & \text { missing } & 17.13 & 23.00 & 6\end{array}$

Eta $^{2}: .14(p<.001)$

Appendix 23 Mean costs of drugs of patients in severity categories representing the extent of Cardiac Wall Motion Abnormalities.

$\begin{array}{llrrr}\text { Category } & \text { Description } & \text { Mean } & \text { Std.dev. } & \text { Freq. } \\ 0 & \text { no wall motion abnormalities } & 54.21 & 34.29 & 22 \\ 1 & \text { wall motion abn. in 1 or 2 wall segments } & 61.65 & 38.28 & 126 \\ 2 & \text { wall motion abn. in 3 wall segments } & 79.56 & 51.31 & 87 \\ 3 & \text { wall motion abn. in 4 wall segments } & 86.48 & 61.46 & 37 \\ 4 & \text { wall motion abn. in 5 wall segments } & 123.43 & 81.32 & 20 \\ 5 & \text { wall motion abn. in 6 or 7 wall segments } & 61.93 & 54.23 & 17 \\ & \text { missing } & 52.90 & 55.22 & 147\end{array}$

$E t a^{2}: . I I(p<.001)$ 
Appendix 24 Mean cost of drugs of patients in severity categories representing type and time of appearance of cardiac rhythm disorders.

\begin{tabular}{|c|c|c|c|c|}
\hline Category & Description & Mean & Std.dev. & Freq. \\
\hline$a$ & no rhythm disorder & 54.02 & 40.14 & 220 \\
\hline$b$ & acute sinustachycardia & 59.00 & 39.60 & 104 \\
\hline$c$ & acute atrial flutter/-fibrillations & 79.50 & 33.88 & 21 \\
\hline$d$ & acute ventricular flutter/-fibrillations & 58.05 & 40.22 & 17 \\
\hline$e$ & $\begin{array}{l}\text { sinustachycardia or non-sustained veniricular } \\
\text { tachy-cardia in follow-up phase }\end{array}$ & 74.11 & 43.30 & 18 \\
\hline$f$ & $\begin{array}{l}\text { recurrent sinustachycardia or non-sustained } \\
\text { ventricular tachycardia }\end{array}$ & 90.39 & 60.73 & 25 \\
\hline$g$ & $\begin{array}{l}\text { acute } A F / F \text { combined with sinustachycardia or } \\
\text { non-sustained ventricular tachycardia in } \\
\text { follow-up plase }\end{array}$ & 74.91 & 46.83 & 6 \\
\hline$h$ & recurrent $A F / F$ & 111.48 & 85.42 & 30 \\
\hline$i$ & $\begin{array}{l}\text { acute } V F / F \text { combined with } A F / F \text { or } \\
\text { sinustachycardia in follow.up phase }\end{array}$ & 124.44 & 134.49 & 7 \\
\hline j & $\begin{array}{l}\text { acute sinustachycardia or AF/F combined with } \\
V F / F \text { or sustained ventricular tachycardia } \\
\text { in follow-up phase }\end{array}$ & 205.28 & 56.06 & 7 \\
\hline & missing & 8.10 & .00 & 1 \\
\hline $\begin{array}{l}E t u^{2}: 2 l \\
A F / F=A l \\
V F / F=V t\end{array}$ & $\begin{array}{l}\text { <.ol) } \\
\text { ial Flatter/-Fibrillations } \\
\text { ntricular Flutrer/-Fibrillarions }\end{array}$ & & & \\
\hline
\end{tabular}

Appendix 25 Mean costs of drugs of patients in severity categories representing the Left Ventricular Ejection Fraction.

$\begin{array}{llrrr}\text { Category } & \text { Description } & \text { Mean } & \text { Siddev. } & \text { Freq. } \\ & & & & \\ 0 & \text { normal }(\text { LVEF }>40 \%) & 58.52 & 35.10 & 183 \\ 1 & \text { slightly impaiment }(30 \%<L V E F<40 \%) & 87.59 & 53.18 & 78 \\ 2 & \text { severe impaiment }(20 \%<L V E F<30 \%) & 110.90 & 75.79 & 47 \\ 3 & \text { very severe impaiment }(L V E F<20 \%) & 67.01 & 65.47 & 8 \\ & \text { missing } & 50.65 & 54.05 & 140\end{array}$

$E t a^{2}: 14(p<.001)$ 
Appendix 26 Correlation matrix of severity of illness indicators, background variables, length of stay and costs of drugs ( $L$ istwise deletion of missing cases, $n=446$ ).

\begin{tabular}{|c|c|}
\hline$\xi$ & 8 \\
\hline$\underline{\underline{2}}$ & $8 \div$ \\
\hline gั & $8 \div \vdots$ \\
\hline z & $8 \div 5$ \\
\hline$\frac{\not}{8}$ & 8 웅 $\dot{2}$ : \\
\hline$\frac{1}{x}$ & $8: \dot{0} 8 \div 2$ \\
\hline$\frac{5}{2}$ & $8 \div 5 \overline{8} \div 8 \dot{4}$ \\
\hline 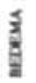 & 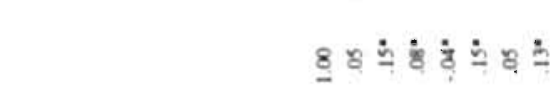 \\
\hline 焉 & 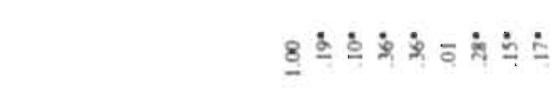 \\
\hline 总 & 8 웅 웡 \\
\hline$\underbrace{\frac{z}{c}}_{0}$ & 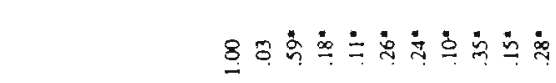 \\
\hline$\frac{3}{2}$ & 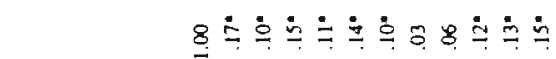 \\
\hline $\bar{\xi}$ & 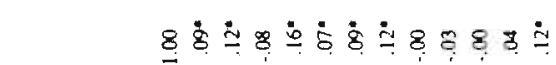 \\
\hline$\sum_{3}^{2}$ & 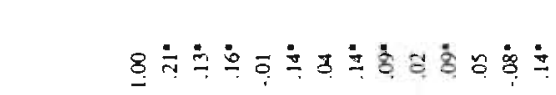 \\
\hline$\frac{\gamma}{2}$ & 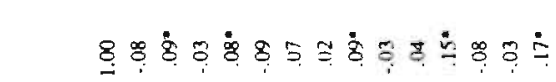 \\
\hline 高 & 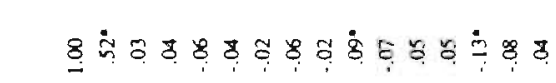 \\
\hline 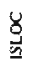 & 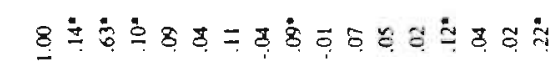 \\
\hline 啇 & 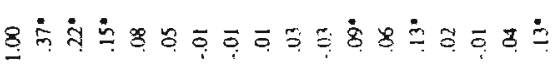 \\
\hline$\frac{\partial}{\partial}$ & 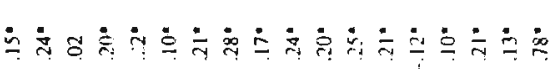 \\
\hline & 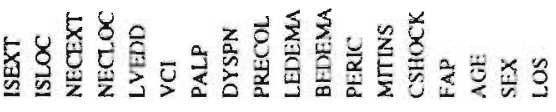 \\
\hline
\end{tabular}


Appendix 27 Results from regression analysis: dependent variable is total costs of drugs in AMI-patients (Listwise deletion of missing cases, $n=456$ ).

\begin{tabular}{|c|c|c|c|c|}
\hline Independent Variables & $B$ & $S B B$ & Beta & Sig T \\
\hline DISPAIN & .93 & 13.08 & .00 & $N S$ \\
\hline DISPAINI & .87 & 4.77 & .00 & $N S$ \\
\hline DISPAIN2 & 11.34 & 5.54 & .09 & .041 \\
\hline DRHYTHMI & -5.82 & 3.41 & -.05 & $N S$ \\
\hline DRHYTHM 2 & 3.85 & 6.49 & .02 & NS \\
\hline DRHYTHMЗ & -1.71 & 7.28 & .00 & NS \\
\hline DRHYTHMA & -4.19 & 6.95 & -.02 & NS \\
\hline DRHYTHMS & -3.57 & 6.30 & .02 & NS \\
\hline DRHYTHMO & .19 .62 & 11.99 & .04 & NS \\
\hline DRHYTHM7 & -.24 & 5.89 & .00 & NS \\
\hline DRHYTHMB & -4.51 & 11.58 & .01 & NS \\
\hline DRHYTHM9 & 32.07 & 11.71 & .07 & .006 \\
\hline DECHO & -7.45 & 3.12 & .07 & $.018^{\prime}$ \\
\hline$\angle V E D D$ & 18.1! & 5.20 & .09 & .001 \\
\hline$B E D E M A$ & 12.34 & 5.06 & .06 & .015 \\
\hline LOS & 7.43 & .43 & .85 & .0001 \\
\hline DADINTO & .34 .06 & 7.99 & $-\| l l$ & .000 \\
\hline DADEXTI & 5.30 & 12.28 & $.0 l$ & NS \\
\hline DADEXT2 & .8 .43 & 10.76 & -.02 & NS \\
\hline DADEXT 3 & 4.57 & 17.35 & .00 & NS \\
\hline DHOMEI & 1.78 & 11.96 & .00 & $N S$ \\
\hline DHOMEJ & .99 & 4.11 & .00 & $N S$ \\
\hline DTRANSI & -87.57 & 19.86 & -.11 & .000 \\
\hline DTRANS2 & .247 .47 & 241.45 & -.30 & .000 \\
\hline DTRANS3 & -38.81 & 28.49 & .03 & NS \\
\hline DDEATHO & 1.44 & 10.03 & .00 & NS \\
\hline DDEATHI & 6.85 & 7.35 & .03 & NS \\
\hline DDEATH2 & 9.28 & 8.38 & .03 & NS \\
\hline DDEATH3 & 12.15 & 12.58 & .03 & $N S$ \\
\hline CONSTANT & -10.27 & 13.23 & & $N S$ \\
\hline
\end{tabular}


Appendix 28 Mean costs of Jaboratory services of patients in severity categories representing myocardial necrosis estimated by total CPK release.

\begin{tabular}{llrrr}
\hline Category & Description & Mean & Std.dev: & Freq. \\
0 & no sig. CPK changes & & & \\
1 & $<10000$ & 17.4 .25 & 61.92 & 9 \\
2 & $10000-20000$ & 218.54 & 119.29 & 85 \\
3 & $20000-40000$ & 235.39 & 158.72 & 74 \\
4 & $40000-60000$ & 226.73 & 155.11 & 104 \\
5 & $>60000$ & 274.94 & 174.78 & 56 \\
& missing & 162.39 & 181.33 & 72 \\
& & & & \\
Eta $: .06(p<.001)$ & & & \\
\hline
\end{tabular}

Appendix 29 Mean costs of laboratory services of patients in severity categories representing ischemic pain.

\begin{tabular}{|c|c|c|c|c|}
\hline Category. & Description & Mean & Staldev. & Freq. \\
\hline 0 & no ischemic pain & 131.94 & 117.68 & 55 \\
\hline 1 & ischemic pain in To or TI & 221.31 & 162.62 & 306 \\
\hline 2 & ischemic pain (also) in T2 or T3 & 227.91 & 158.02 & 93 \\
\hline & missing & 122.10 & 179.92 & 6 \\
\hline
\end{tabular}

Appendix 30 Mean costs of laboratory services of patients in severity categories representing the extent of in Cardiac Wall Motion abnormalities.

\begin{tabular}{llrrr}
\hline Category Description & Mean & Sid.dev. & Freq. \\
& & & & \\
1 & no wall motion abnomalities & 173.07 & 104.76 & 22 \\
2 & wall motion abn. in 1 or 2 wall segments & 217.45 & 154.71 & 126 \\
3 & wall motion abn. in 3 wall segments & 243.71 & 162.85 & 88 \\
4 & wall motion abn. in 4 wall segments & 246.98 & 189.46 & 40 \\
5 & wall motion abn. in 5 wall segments & 330.26 & 231.86 & 21 \\
& wall motion abn. in 6 or 7 wall segments & 191.34 & 113.78 & 16 \\
& missing & 165.83 & 136.90 & 147 \\
Eta & & & \\
\hline
\end{tabular}


Appendix 31 Mean costs of laboratory services of patients in severity categories representing type and time of appearance of cardiac rhythm disorders.

\begin{tabular}{|c|c|c|c|c|}
\hline Category & Description & Mean & Std.dev. & Freq. \\
\hline$a$ & no rhythm disorder & $j 73.46$ & 135.60 & 222 \\
\hline$b$ & acute sinustachycardia & 223.36 & 166.30 & 130 \\
\hline$c$ & acute atrial flutter/-fibrillations & 232.65 & 149.41 & 21 \\
\hline$d$ & acute vensticular flutter/-fibrillations & 202.43 & 157.45 & 18 \\
\hline e & $\begin{array}{l}\text { simustachycanlia or non-sustained ventricular } \\
\text { tachycardia in follow-ip phase }\end{array}$ & 271.19 & 170.61 & 18 \\
\hline$f$ & $\begin{array}{l}\text { recurrent sinustachycardia or non-sustained } \\
\text { ventricular tachycardia }\end{array}$ & 249.30 & 130.99 & 26 \\
\hline$g$ & $\begin{array}{l}\text { acule } A F / F \text { combined with sinustachycardia or } \\
\text { non-sustained ventricular tachycardia in } \\
\text { follow-up phase }\end{array}$ & 239.37 & 266.93 & 5 \\
\hline$h$ & recurrent $A F / F$ & 232.70 & 141.14 & 30 \\
\hline$i$ & $\begin{array}{l}\text { acute VF/F combined with AF/F or sinustachy- } \\
\text { cardia in follow-up phase }\end{array}$ & 372.78 & 187.73 & 7 \\
\hline \multirow[t]{2}{*}{ i } & $\begin{array}{l}\text { simustachycardia or } A F / F \text { combined with } V F / F \\
\text { or sustained ventricular tachycarlia in follow-up } \\
\text { phase }\end{array}$ & 520.74 & 231.88 & 9 \\
\hline & missing & 25.40 & 0.00 & 1 \\
\hline $\begin{array}{l}E t a^{2}: I 31 \\
A F / F=A t \\
V F / F=V e\end{array}$ & $\begin{array}{l}\text { <.(01) } \\
\text { rial Flutter/-Fibrillations } \\
\text { niricular Flutter/.Fibrillations }\end{array}$ & & & \\
\hline
\end{tabular}


Appendix 32 Correlation matrix of severity of illness indicators, background variables, length of stay and enrollment in PRIMI protocol and costs of Iaboratory services (Listwise deletion of missing cases, $n=456$ ).

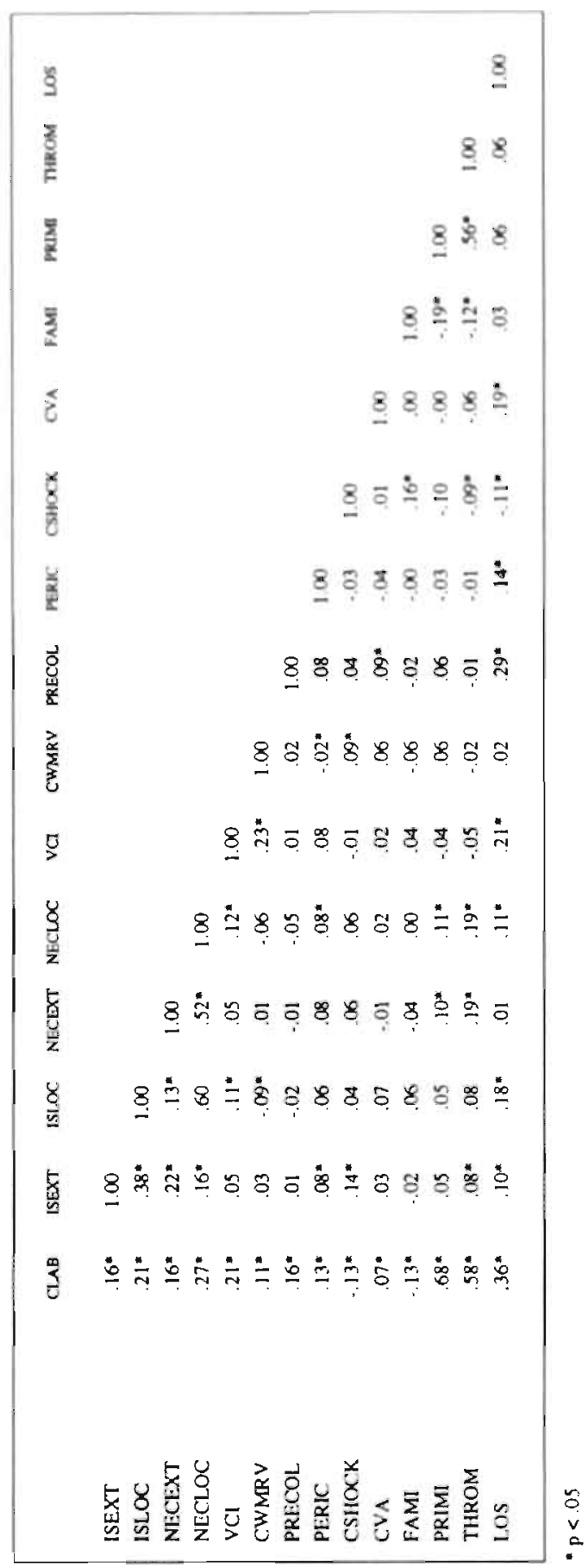


Appendix 33 Results from regression analysis: dependent variable is total costs of laboratory services (Listwise deletion of missing cases, $n=458$ ).

\begin{tabular}{|c|c|c|c|c|}
\hline Independent Variables & $B$ & $S E B$ & Beta & $\operatorname{Sig} T$ \\
\hline NECLOC & 37.88 & 7.69 & .15 & .000 \\
\hline$D C P K$ & -66.31 & 34.85 & -.15 & NS \\
\hline$D C P K I$ & 62.32 & 34.16 & .15 & NS \\
\hline DCPK2 & 76.08 & 34.72 & .18 & .029 \\
\hline$D C P K 3$ & 64.54 & $34.38^{\prime}$ & .17 & NS \\
\hline$D C P K 4$ & 63.05 & 35.36 & .13 & NS \\
\hline DCPKS & 96.92 & 35.50 & .20 & .007 \\
\hline DISPAIN & -76.38 & 46.60 & .05 & NS \\
\hline DISPAINI & 41.15 & 16.82 & .12 & .015 \\
\hline DISPAIN2 & 43.36 & 18.90 & .11 & .022 \\
\hline DRHYTHMI & 15.23 & 11.34 & .04 & NS \\
\hline DRHYTHM 2 & 10.75 & 21.87 & .01 & NS \\
\hline DRHYTHM3 & 5.25 & 23.92 & .00 & NS \\
\hline DRHYTHMA & 3.43 & 22.94 & .00 & NS \\
\hline DRHYTHMS & 17.45 & 19.89 & .03 & NS \\
\hline DRHYTHMO & .47 & 41.22 & .00 & NS \\
\hline DHRYTHM7 & 36.09 & 18.70 & .06 & NS \\
\hline DRHYTHM & 97.92 & 37.27 & .08 & .009 \\
\hline DRHYTHMS & 176.62 & 34.21 & .15 & .000 \\
\hline$D C O N D U C I$ & -12.93 & 28.46 & -.01 & NS \\
\hline DCONDUC2 & 23.17 & 16.44 & .04 & NS \\
\hline DCONDUC3 & -1.99 & $29.9 ?$ & .00 & $N S$ \\
\hline DCONDUCA & 78.73 & 31.75 & .08 & .014 \\
\hline DCONDUCS & -1.49 & 23.22 & .00 & NS \\
\hline DCONDUCO & 46.21 & 48.15 & .03 & NS \\
\hline DECHO & -.45 & 10.51 & .00 & NS \\
\hline$V C I$ & 61.44 & 17.07 & .10 & .000 \\
\hline$C V A$ & $14 J .23$ & 40.63 & .12 & $.00 \mathrm{I}$ \\
\hline PRIMI & 302.37 & 13.55 & .63 & .000 \\
\hline $\operatorname{LOS}$ & 3.51 & .66 & $2 !$ & .000 \\
\hline DADINTO & .57 .03 & 25.67 & -.06 & .027 \\
\hline DADEXT! & .17 .99 & 44.05 & -.01 & NS \\
\hline DADEXT2 & 7.75 & 35,96 & .00 & NS \\
\hline DADEXT3 & -9.33 & 55.61 & .00 & N.S \\
\hline DHOMEI & 43.61 & 38.85 & .03 & $N S$ \\
\hline DHOME3 & 18.02 & 11.62 & .06 & $N: S$ \\
\hline DTRANSI & -152.85 & 60.10 & -.08 & .011 \\
\hline DTRANS2 & -196.52 & 57.37 & -.12 & .001 \\
\hline DTRANS3 & 95.38 & 91.32 & .03 & NS \\
\hline DDEATHO & .116 .26 & 36.96 & .10 & .002 \\
\hline DDEATHI & .76 .50 & 27.85 & .03 & NS \\
\hline DDEATH2 & 49.41 & 27.16 & .05 & NS \\
\hline DDEATH 3 & -27.51 & 38.55 & -.02 & NS \\
\hline CONSTANT & 104.15 & 45.48 & & .023 \\
\hline
\end{tabular}

Explained Variance $\left(\boldsymbol{R}^{2}\right): .72$ 
Appendix 34 Mean costs of ECG of patients in severity categories representing myocardial necrosis estimated by total CPK release.

$\begin{array}{lllrr}\text { Category Description } & \text { Mean } & \text { Std.dev. } & \text { Freq. } \\ & & & & \\ 0 & \text { no sig. CPK changes } & 24.03 & 27.50 & 9 \\ 1 & <10000 & 35.84 & 23.32 & 85 \\ 2 & 10000-20000 & 48.14 & 28.40 & 72 \\ 3 & 20000-40000 & 49.98 & 29.09 & 106 \\ 4 & 40000 \cdot 60000 & 48.47 & 34.09 & 58 \\ 5 & >60000 & 64.51 & 43.74 & 56 \\ & \text { missing } & 42.73 & 57.16 & 71\end{array}$

Eta $: .08(p<.001)$

Appendix 35 Mean costs of ECG of patients in severity categories representing ischemic pain.

Category Description

o no ischemic pain

I ischemic pain in To or TI

2 ischemic pain (also) in T2 or T3

missing
Mean

29.92

44.25

68.23

15.90
Sid.dev. Freq.

$23.69 \quad 54$

$33.43 \quad 305$

$46.33 \quad 92$

$23.71 \quad 6$

$E 1 a^{2}: .19(p<.001)$

Appendix 36 Mean costs of ECG of patients in severity categories representing the extent of Cardiac Wall Motion Abnormalities.

\section{Category Description}

0

I

2

3

4

5 no wall motion abnormalities

wall motion abn. in 1 or 2 wall segments

wall motion abn. in 3 wall segments

wall motion abn. in 4 wall segments

wall motion abn. in 5 wall segments

wall motion abn. in 6 or 7 wall segments

missing
Mean

37.87

47.60

57.82

57.95

99.45

39.28

31.67
Sid.dev. Freq.

$13.67 \quad 22$

$25.16 \quad 126$

$37.49 \quad 88$

$38.13 \quad 36$

$76.84 \quad 22$

$42.56 \quad 17$

$27.73 \quad 146$

$E q a^{2}: .13(p<.001)$ 
Appendix 37 Mean costs of ECG of patients in severity categories representing type and time of appearance of cardiac rhythm disorders.

Category Description

a no rhythm disorder

b acute sinustachycardia

c acute atrial flutter/-fibrillations

d acute ventricular flutter/-fibrillations

$\ell \quad$ sinustachycardia or non-sustained ventricular

tachycardia in follow-up phase

$f$ recurrent sinustachycardia or non-sustained

ventricular tachycardia

g acute $A F / F$ combined with sinustachycardia or non-sustained ventricular tachycardia in

follow-up phase

h recurreni $A F / F$

i acuse $V F / F$ combined with $A F / F$ or sinustachy= cardia in follow-up phase

acute sinustachycardia or $A F / F$ combined

with $V F / F$ or sustained ventricular tachycardia

in follow-up phase

missing
Mean

36.22

43.24

50.88

38.91

62.19

63.35

39.27

33.47

5

75.09

55.41

31

64.66

39.91

6

168.19

82.99

9

0.00

0.00

$I$

\section{0}

21

17

18

25

$E t a^{2}: .32(p<.001)$

$A F / F=$ Atrial Flutier/-Fibrillations

$V F / F=$ Ventricular Flutter/.Fibrillations.s

Appendix 38 Mean costs of ECG of patients in severity categories representing Left Ventricular Fjection Fraction.

Category Description

normal $($ LVEF $>40 \%)$

slightly impaiment $(30 \%<L V E F<40 \%)$

severe impairment $(20 \%<L V E F<30 \%)$

very severe impairmen ( $L V E F<20 \%$ )

missing
Mean

44.35

67.65

72.45

53.71

29.79
Siddev. Freq.

20.57

183

49.90

80

43.62

46

97.76

25.50

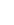

1

6


Appendix 39 Correlation matrix of severity of illness indicators, background variables, length of stay and costs of ECG (Listwise deletion of missing cases, $\mathrm{n}=451$ ).

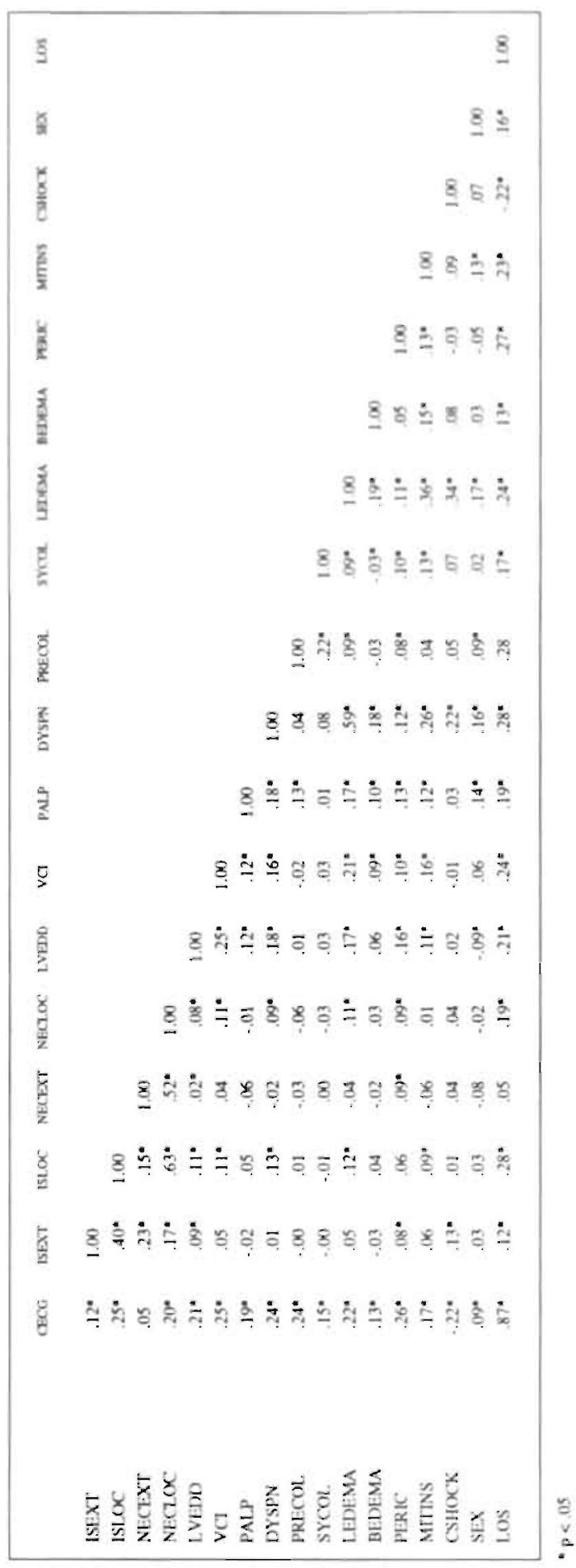


Appendix 40 Results from regression analysis: dependent variable is costs of ECG (Listwise deletion of missing cases, $n=457$ ).

\begin{tabular}{|c|c|c|c|c|}
\hline Independent Variables & $B$ & $S E B$ & Beta & $\operatorname{Sig} T$ \\
\hline INRHYTHMI & .42 & 1.59 & .00 & $N S$ \\
\hline DRHYTHM2 & 1.79 & 3.04 & .01 & $N S$ \\
\hline DRHYTHM 3 &. .70 & 3.37 & .00 & NS \\
\hline DRHYTHM4 & 7.58 & 3.24 & .04 & .020 \\
\hline DRHYTHMS & 5.40 & 2.91 & .03 & NS \\
\hline DRHYTHMO & 7.89 & 6.00 & .02 & NS \\
\hline DRHYTHM7 & -.38 & 2.71 & .00 & NS \\
\hline DRHYTHMB & 8.74 & 5.58 & .03 & NS \\
\hline DRHYTHM9 & 19.43 & 5.36 & .07 & .003 \\
\hline $\operatorname{LOS}$ & 5.22 & .17 & .93 & .000 \\
\hline DADINTO & .19 .16 & 3.78 & .09 & .000 \\
\hline DADEXTI & .05 & 5.53 & .00 & NS \\
\hline DADEXT2 & 6.05 & 4.73 & .02 & NS \\
\hline DADEXT3 & 8.38 & 7.85 & .02 & NS \\
\hline DHOMEI & .4 .78 & 5.52 & .02 & $N S$ \\
\hline DHOME3 & .17 & 1.83 & -.00 & NS \\
\hline DTRANSI & .67 .13 & 9.29 & .12 & .000 \\
\hline DTRANS2 & -169.52 & 10.87 & -.30 & .000 \\
\hline DTRANS3 & -16.52 & 13.28 & -.02 & NS \\
\hline DDEATHO & 1.79 & 4.39 &.$\infty$ & NS \\
\hline DDEATHI & .354 & 3.31 &. .02 & NS \\
\hline DDEATH2 & -9.66 & 3.83 & .05 & .012 \\
\hline I)I)EATH3 & 1.06 & 5.84 & .00 & NS \\
\hline 1) CONSTANT & -8.66 & 1.67 & & .000 \\
\hline
\end{tabular}

Appendix 41 Mean costs of echocardiography of patients in severity categories representing myocardial necrosis estimated by total CPK release.

\begin{tabular}{llrrr}
\hline Category Description & Mean & Siddev. & Freq. \\
0 & no sig. CPK changes & & & \\
1 & $<10000$ & 31.72 & 31.78 & 9 \\
2 & $10000 \cdot 20000$ & 35.86 & 32.59 & 85 \\
3 & 20000.40000 & 39.82 & 28.38 & 74 \\
4 & $40000-60000$ & 48.13 & 23.01 & 106 \\
5 & $>60000$ & 44.90 & 31.67 & 60 \\
& missing & 59.95 & 31.11 & 56 \\
& & 37.39 & 44.60 & 74 \\
Eta? $: 07(p<.001)$ & & & \\
\hline
\end{tabular}


Appendix 42 Mean costs of echocardiography of patients in severity categories representing type and time of appearance of cardiac rhythm disorders.

\begin{tabular}{|c|c|c|c|c|}
\hline Category & Description & Mean & Std dev: & Freq. \\
\hline$a$ & no rhythm disorder & 36.73 & 27.73 & 222 \\
\hline$b$ & acute sinushchycardia & 40.30 & 26.52 & 105 \\
\hline$c$ & acute atrial flutter/-fibrillations & 40.78 & 28.70 & 21 \\
\hline$d$ & acute ventricular flutter/-fibrillations & 47.58 & 35.07 & 18 \\
\hline$e$ & $\begin{array}{l}\text { sinustachycardia or non-sustained ventricular } \\
\text { tachycardia in follow-up phase }\end{array}$ & 49.63 & 22.65 & 18 \\
\hline$f$ & $\begin{array}{l}\text { recurrent sinustachycardia or non-sustained } \\
\text { ventricular tachycardia }\end{array}$ & 60.57 & 37.36 & 26 \\
\hline$g$ & $\begin{array}{l}\text { acute } A F / F \text { combined with sinustachycardia or } \\
\text { non-sustained ventricular tachycardia in } \\
\text { follow-up phase }\end{array}$ & 56.03 & 34.63 & 6 \\
\hline$h$ & recurrent $A F / F$ & 62.69 & 42.59 & 31 \\
\hline$i$ & $\begin{array}{l}\text { acute } V F / F \text { combined with } A F / F \text { or sinustachy- } \\
\text { cardia in follow-up phase }\end{array}$ & $52: 63$ & 43.99 & \\
\hline j & $\begin{array}{l}\text { acute sinustachycardia or } A F / F \text { combined with } \\
V F / F \text { or sustained ventricular tachycardia in } \\
\text { follow-up phase }\end{array}$ & 109.50 & 57.79 & \\
\hline & missing & 0.00 & 0.00 & \\
\hline
\end{tabular}

$E t a^{2}: .12(p<.001)$

$A F / F=$ Atrial Flutter/-Fibrillations

$V F / F=$ Ventricular Flutter/-Fibrillations 
Appendix 43 Correlation matrix of severity of illness indicators, background variables, length of stay and costs of echocardiography (Listwise deletion of missing cases, $n=446$ ).

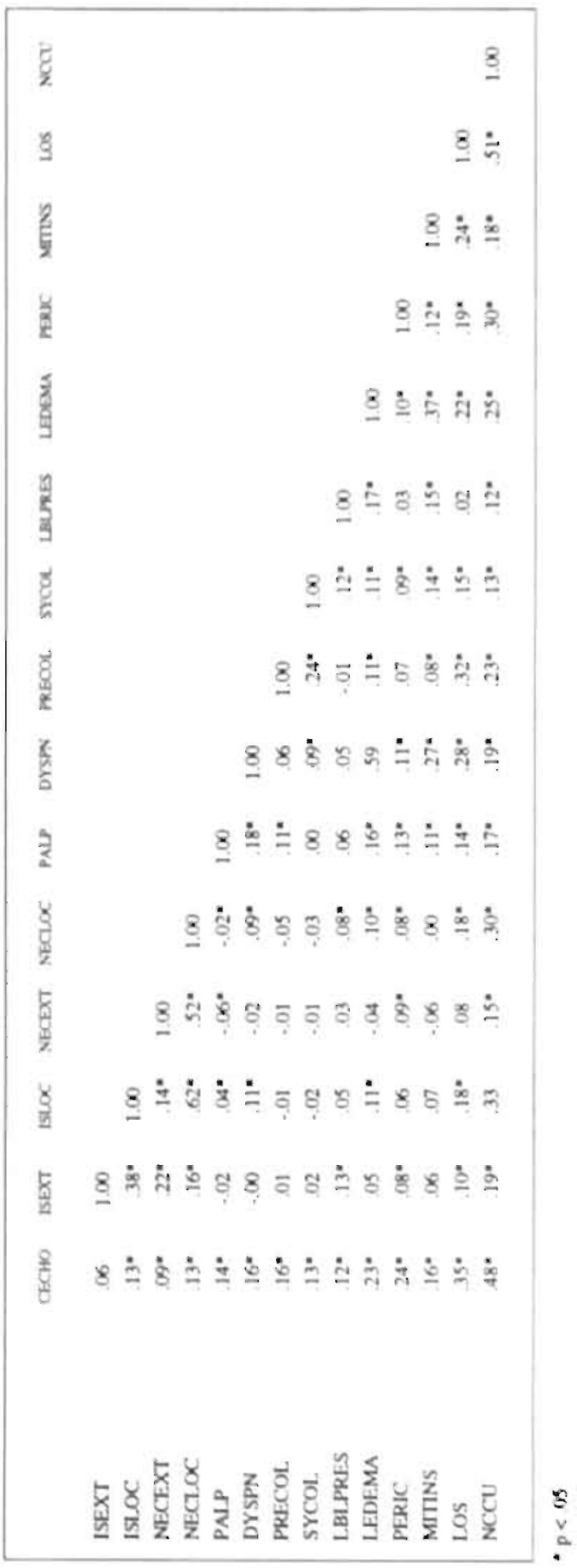


Appendix 44 Results from regression analysis: dependent variable is costs of echocardiography

(Listwise deletion of missing cases, $n=461$ ).

Independent
LEDEMA
PERIC
NCCU
LOS
DADINT0
DADEXT1
DADEXT2
DADEXT3
DHOMEI
DHOME3
DTRANSI
DTRANS2
DTRANS3
DDEATHO
DDEATHI
DDEATH2
DDEATH3
CONSTANT

Explained Variance $\left(R^{2}\right): .32$

$\begin{array}{rrrr}B & \text { SE B } & \text { Beta } & \text { Sig T } \\ 5.58 & 1.94 & .13 & .004 \\ 11.91 & 4.67 & .11 & .011 \\ 1.96 & .30 & .33 & .000 \\ .34 & .20 & .10 & \mathrm{NS} \\ -7.08 & 7.58 & -.04 & \mathrm{NS} \\ -8.29 & 11.50 & .03 & \mathrm{NS} \\ -5.18 & 9.91 & -.02 & \mathrm{NS} \\ 2.87 & 16.30 & .00 & \mathrm{NS} \\ -35.29 & 11.57 & -.12 & .002 \\ .50 & 3.41 & .00 & \mathrm{NS} \\ -10.40 & 16.48 & -.03 & \mathrm{NS} \\ -41.30 & 16.18 & -.12 & .011 \\ 59.19 & 28.02 & .08 & .035 \\ -13.96 & 10.78 & -.05 & \mathrm{NS} \\ -23.19 & 6.99 & -.14 & .001 \\ 10.37 & 7.97 & .05 & \mathrm{NS} \\ -14.85 & 11.18 & .06 & \mathrm{NS} \\ 29.26 & 2.41 & & .000\end{array}$


Appendix 45 Mean costs of exercise tests of patients in severity categories representing by type and time appearance of cardiac rhythm disorders.

Category Description

a no rhythm disorder

b acute sinustachycardia

c acute atrial flutter/-fibrillations

d acute ventricular flutterifibrillations

e sinustachycandia or non-sustained ventricular

tachycardia in follow-up phase

$f \quad$ recurrent sinustachycardia or non-sustained venericular tachycardia

g acute $A F / F$ combined with sinusiachycardia or non-sustained ventricular tachycardia in follow-up phase

h recurrent $A F / F$

$i$ acute $V F / F$ combined with $A F / F$ or

sinustachycardia in follow-up phase

j acure sinustachycardia or $A F / F$ combined with

$V F / F$ or sustained ventricular tachycandia in follow-up phase

missing
Mean Std.dev. Freq.

50.83

52.80

222

68.06

76.73

104

44.18

59.58

21

45.51

61.47

18

53.43

43.13

18

41. 41

44.15

26

55.34

74.92

6

2l.2!

36.68

30

35.48

78.14

7

48.26

53.56

8

0.00

0.00

$E t a^{2}: 04(p<.05)$

$A F / F=$ Atrial Flutter/ $-F$ ibrillations

$V F / F=$ Ventricular Flutter/Fibrillations

Appendix 46 Mean costs of exercise tests of patients in severity categories representing the Left Ventricular Fjection Fraction.

Category Description

o) normal ( $L V E F>40 \%)$

1 slightly impairment $(30 \%<L V E F<40 \%)$

2 severe impairment $(20 \%<L V E F<30 \%)$

3
Mean

59.98

72.28

36.60

21.37

36.03
Sid.dev. Freq.

55.05

78.67

53.87

33.88

49.60
183

79

49

9

I4I

Eta ${ }^{2}: .04(p<.01)$ 
Appendix 47 Correlation matrix of severity of illness indicators, background variables, length of stay and costs of exercise tests (Listwise deletion of missing cases, $n=453$ ).

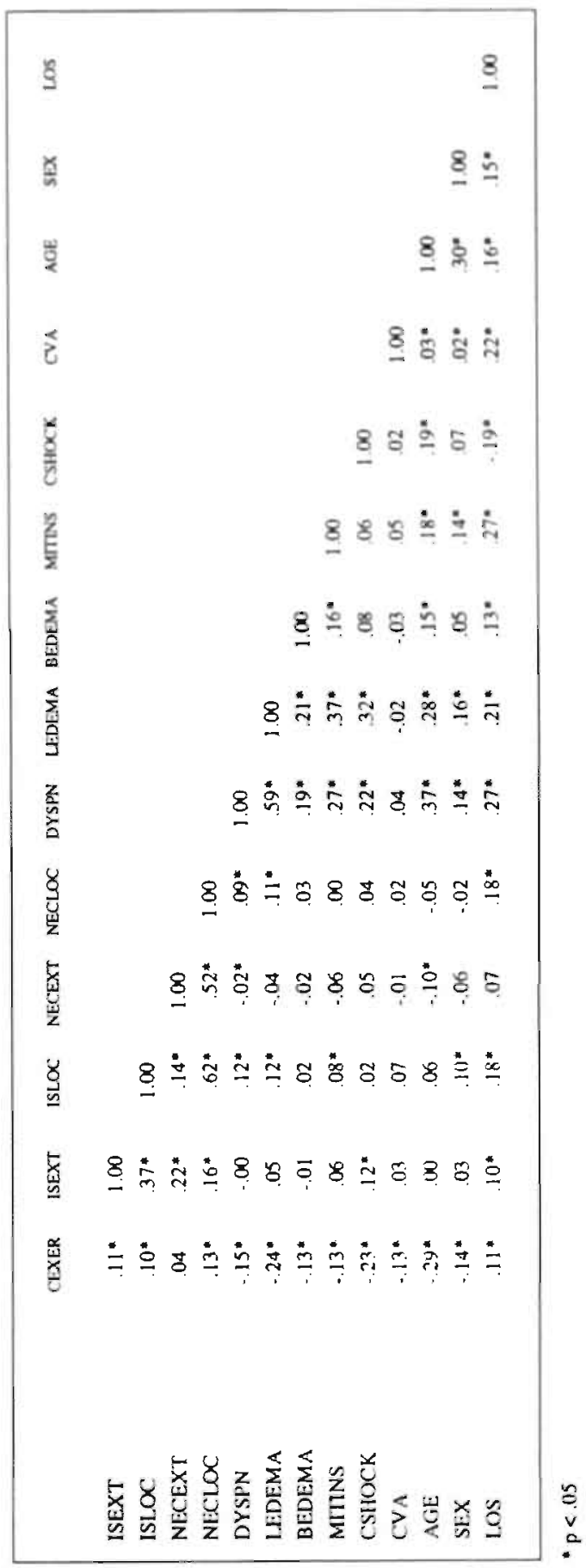


Appendix 48 Results from regression analysis: dependent variable is cosits of exercise ECG (Listwise deletion of missing cases, $n=460$ ).

\begin{tabular}{|c|c|c|c|c|}
\hline Independent Variables & $B$ & $S E B$ & Beta & Sig 1 \\
\hline DRHYTHMI & 11.42 & 6.00 & .09 & $.058(N S)$ \\
\hline DRHYTHM2 & -2.32 & 11.65 & .00 & NS \\
\hline DRHYTHM 3 & -6.24 & 12.49 &. .02 & NS \\
\hline DRHYTHMA & -10.07 & 12.18 & .04 & NS \\
\hline DRHYTHMS & -11.15 & 10.68 & .05 & NS \\
\hline DRHYTHMO & 2.56 & 20.98 & .00 & NS \\
\hline DRHYTHM7 & -23.47 & 10.19 & -.10 & .022 \\
\hline DRHYTHMS & -10.35 & 19.84 &. .02 & NS \\
\hline DRHYTHMS & -10.87 & 19.03 &. .03 & NS \\
\hline BEDEMA & -18.61 & 8.81 & .09 & .035 \\
\hline$C V A$ & -42.60 & 21.03 & -.11 & .043 \\
\hline$A G E$ & -1.07 & .22 & $=.23$ & .000 \\
\hline LOS & .02 & .35 & .00 & NS \\
\hline DADINTO & 12.70 & 13.62 & .04 & NS \\
\hline DADEXTI & -5.16 & 20.99 & -.01 & NS \\
\hline DADEXT2 & 41.25 & 17.65 & .10 & .020 \\
\hline DADEXT3 & -27.87 & 28.96 & .04 & NS \\
\hline DHOMEI & -59.77 & 20.64 & -.12 & .004 \\
\hline DHOME3 & 23.44 & 5.90 & .21 & .000 \\
\hline DTRANSI & -7.19 & 41.45 & .00 & NS \\
\hline ITRANS2 & 6.19 & 30.75 & .01 & NS \\
\hline DDEATHO & 42.59 & 16.31 & $\because 11$ & .009 \\
\hline IDEATHI & 41.76 & 12.34 & -.15 & .001 \\
\hline DDEATH 2 & -31.64 & 14.40 & -.10 & .029 \\
\hline DI)EATH3 & 10.85 & 21.96 & .02 & NS \\
\hline CONSTANT & 116.51 & 13.87 & & .000 \\
\hline
\end{tabular}

Explained Variance $\left(R^{y}\right) ; .25$ 


\section{Appendix 49 Pattern-analysis}

$(*=$ adjacent paftern)

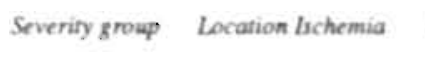

Ischemic Pain

Compli.
cations

Frry.

I

$\boldsymbol{o}$

0

0

I.VEF

0

108

108

2

1

0
0

0
0

41
17
58

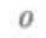

0

0

o

12

4

$\begin{array}{ll}0 & 0 \\ 0 & 0 \\ 0 & 0 \\ 0 & 0\end{array}$

5

6

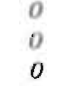

0
0
0

1
0
1
0

I

o

o

12

42
14
5
2
63

7

o

0

0
1
0
$l$

0
0
1
1

o

$\frac{13}{13}$

8

$\begin{array}{ll}1 & 0 \\ 0 & 1 \\ 1 & 0 \\ 0 & 1 \\ 1 & 0 \\ 0 & 1 \\ 1 & 0 \\ 0 & 1\end{array}$

9

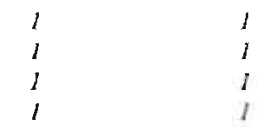

10

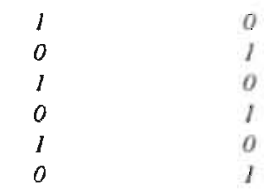

II

0
$o$
0

0
0
0

1
2
2

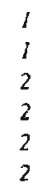

0
0
0
0
0
0

5
0
5
3
1
1
15

2
8
0
10 
Severify gmup Location Ischemia Ischemic Pain Rhythm Disorders

Compit. Freq. cations

12

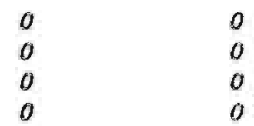

1
2
1
2

2

3
2
1
2
8

13

1
0
1
0
1
0

0
1
0
1
0
1

$\begin{array}{ll}1 & 1 \\ 1 & 1 \\ 2 & 0 \\ 2 & 0 \\ 2 & 1 \\ 2 & 1\end{array}$

14

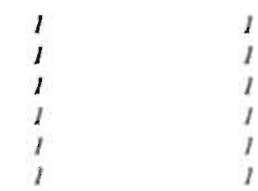

15
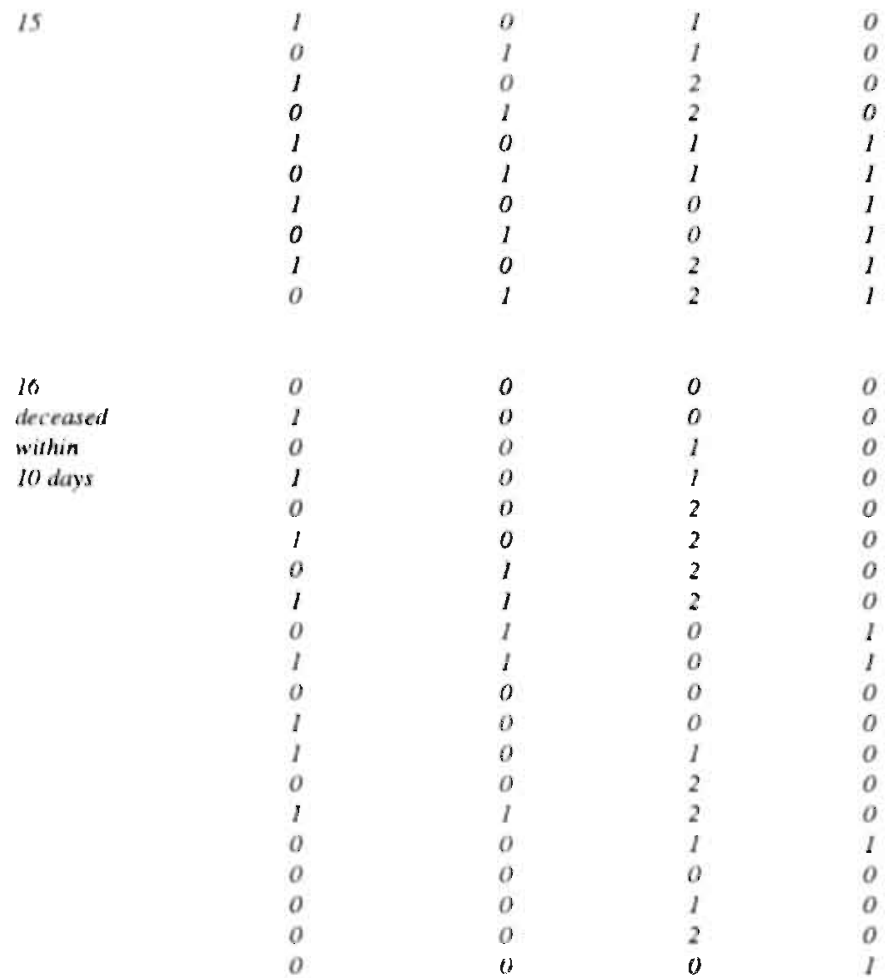

17

deceased afer

10 days

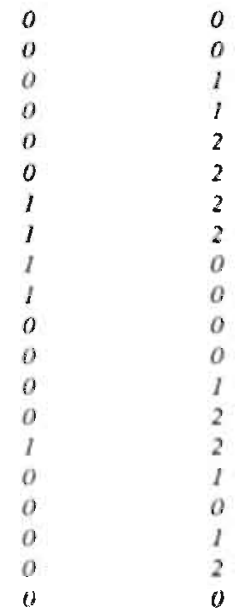

$\begin{array}{ll}0 & 7 \\ 0 & 7 \\ 0 & 5 \\ 0 & 4 \\ 0 & 1 \\ 0 & 1 \\ 0 & 1 \\ 0 & 1 \\ 0 & 2 \\ 0 & 1 \\ 1 & 2 \\ 1 & 1 \\ 1 & 1 \\ 1 & 2 \\ 1 & 1 \\ 1 & 1 \\ 2 & 2 \\ 2 & 1 \\ 2 & 1 \\ 2 & 1 \\ & 43\end{array}$


18.

unclassifiable
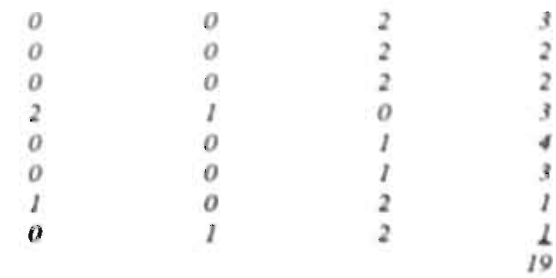

outliers

1
0
1
0
1
0

0
0
2
2
2
1

$\begin{array}{ll}0 & 0 \\ 0 & 2 \\ 1 & 2 \\ 1 & 2 \\ 0 & 2 \\ 0 & 2\end{array}$

Appendix 50 Means, standard deviations and numbers of the SICLOS for AMI-patients by age and sex.

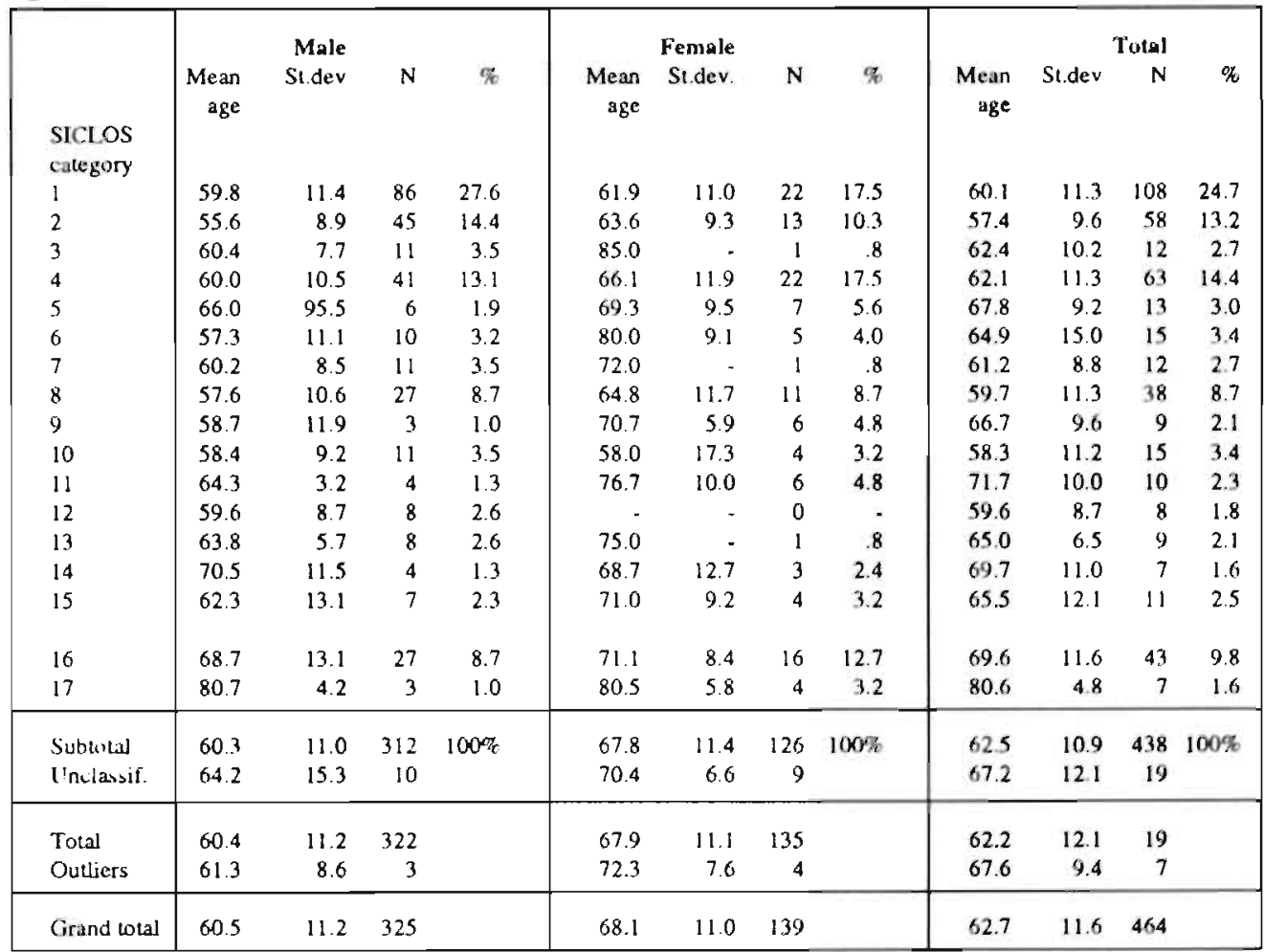





\section{Curriculum Vitae.}

Gemma Voss was born in Susteren on the 12th of november 1961. In 1980 she finished her high-school at the Bisschoppelijk College in Sittard. After graduating from the Faculıy of Health Sciences at the University of Limburg in 1984, she has attended an one-year course in research methodology at the same university. During that year, 1985, she joined the University Hospital Maastricht where she is in charge of the development and implementation of the DRG patient classification system. During 1986 she visited various hospitals and universities in the USA to learn from the experience with patient classification systems. On a number of occasions she was a temporary advisor for the World Health Organization. She has published articles and has given several lectures on the subject of patient classification systems. In 1987 she started her doctoral research. 


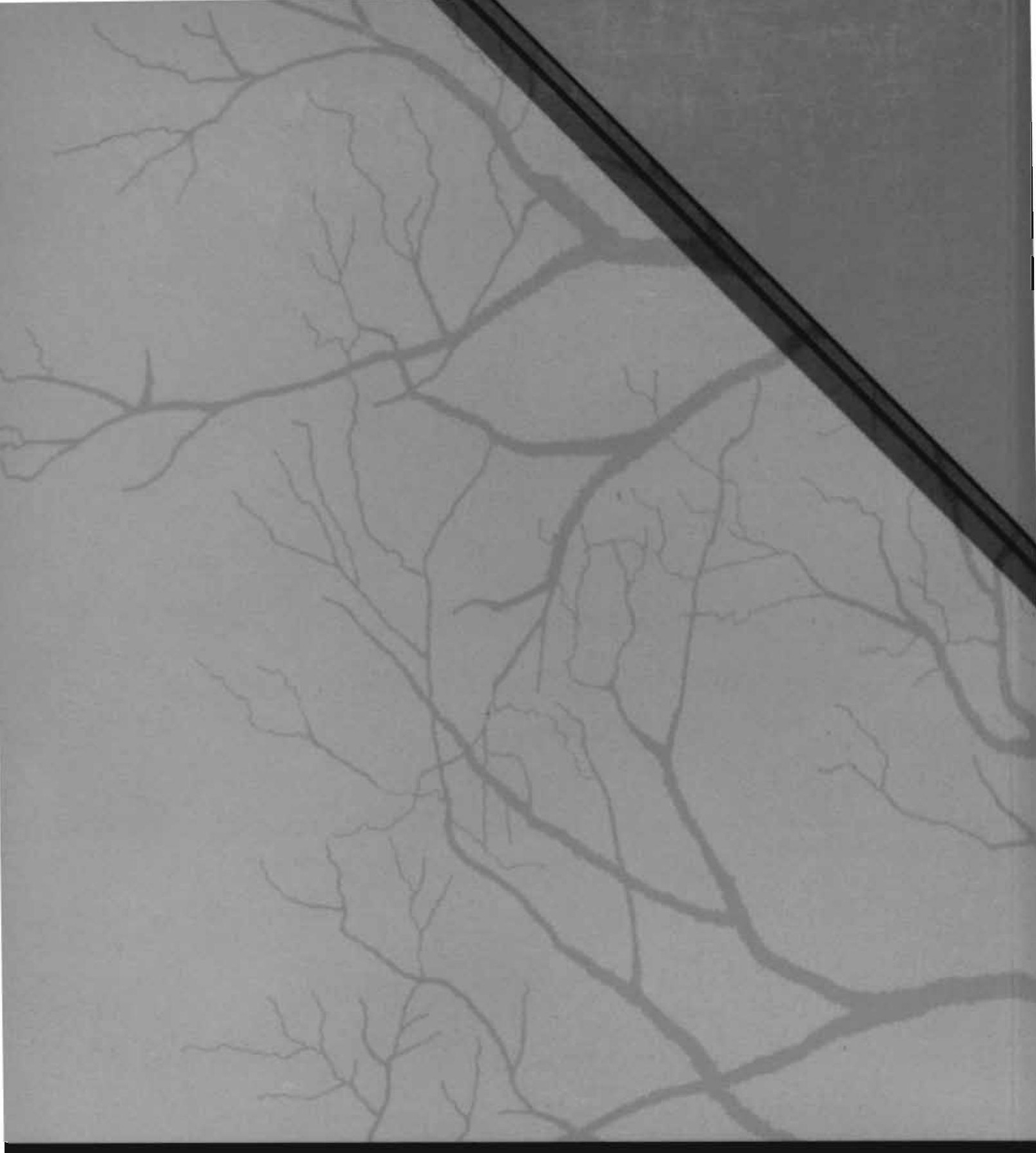

